\title{
Construction of Low-Symmetric and Light-Switchable Coordination Cages for Guest Uptake and Catalysis
}

\author{
Dissertation \\ zur Erlangung des mathematisch-naturwissenschaftlichen Doktorgrades \\ "Doctor rerum naturalium" \\ der Georg-August-Universität Göttingen

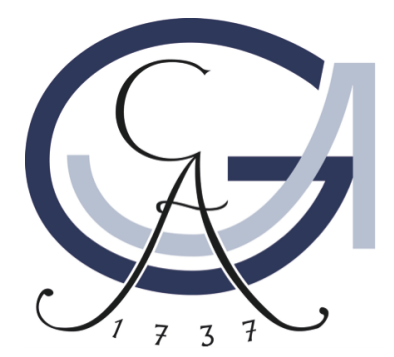 \\ im Promotionsprogramm \\ Catalysis for Sustainable Synthesis

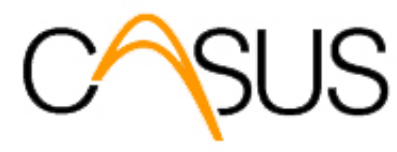 \\ der Georg-August University School of Science (GAUSS)
}

vorgelegt von

Muxin Han

aus Tianjin, VR China

Göttingen, 2015 


\section{Betreuungsausschuss}

Prof. Dr. Guido H. Clever, Institut für Anorganische Chemie

Prof. Dr. Franc Meyer, Institut für Anorganische Chemie

Prof. Dr. Lutz Ackermann, Institut für Organische und Biomolekulare Chemie

\section{Mitglieder der Prüfungskommission}

Referent: $\quad$ Prof. Dr. Guido H. Clever, Institut für Anorganische Chemie

Korreferent: $\quad$ Prof. Dr. Franc Meyer, Institut für Anorganische Chemie

Weitere Mitglieder der Prüfungskommission:

Prof. Dr. Lutz Ackermann, Institut für Organische und Biomolekulare Chemie

Prof. Dr. Dietmar Stalke, Institut für Anorganische Chemie

Prof. Dr. Dirk Schwarzer, Max-Planck-Institut für Biophysikalische Chemie

Dr. Michael John, Institut für Organische und Biomolekulare Chemie

Tag der mündlichen Prüfung: $\quad$ 08.10.2015 
To all the people who had asked me repeatedly over the years,

"when are you going to graduate...?"

To my parents

致我的妈妈爸爸 


\section{ACKNOWLEDGEMENTS}

The completion of my dissertation has been a long journey, it is a both painful and enjoyable experience. I would like to thank many people who have helped me through the completion of this dissertation. Without you, I could not have achieved so much alone.

First and foremost I want to thank my advisor Prof. Guido Clever, who introduced me into the supramolecular chemistry. It has been an honour to be his first PhD student. I appreciate all his contributions of time, ideas, advices, and funding to make my Ph.D. experience productive and stimulating. He has been actively interested in my work, and has given me the freedom to pursue various projects without objection. I would like to thank him for encouraging me, even during tough times in my PhD. His advices on both research as well as my career have been invaluable.

I would like to offer my special thanks to my secondary and third supervisor Prof. Franc Meyer and Prof. Lutz Ackermann for their support during my research. I am thankful to Prof. Dietmar Stalke, Prof. Dirk Schwarzer, Dr. Michal John for being in my defense examination board.

Special thanks are given to Dr. Michael John for the time he has invested for measuring my samples, and for the valuable discussions and suggestions for the elucidation and characterization of my structures. I would like to thank Ralf Schöner and Wolfgang Zolke for the fulfillment of my special wishes and the arrangement of my measuring. I would like to thank Dr. Holm Frauendorf and Ms. Györgyi Sommer-Udvarnoki that have attempted with patience to analyze my samples.

I would like to thank Prof. Dietmar Stalk, Dr. Jakob Hey, Reent Michel, Lennard Krause for the support and measurements of X-ray structures.

I would like to thank my bachelor student Sayaka Shoji, master student Anqi Chen, and all the research students Jan Geweke, Stefan Ortgies, for their time, efforts and contribution to this work.

All brilliant lab colleagues, past and present: Marina, David, Susanne, Fernanda, Rongmei, Macel, Thorben, Rujin, Yoko, Eike, Sabrina, Sabine, Anqi, Sayaka, Nils, Mirco, Kristoff, Ilker, Andreas, Thomas, Sabrina, Anna. Hopefully I did not forget anyone. Thank you for your help and advices, for the laughter and the tears, for all the time we spent.

I am extremely thankful to Dr. Mark Johnstone, Dr. Witold Bloch, Thomas Fallows, Marina Frank, Torben Böhnisch, for proofreading and commenting the present PhD dissertation. 
I thank Matthias Hesse, Martin Schlote for supplying all chemicals and laboratory equipment. I would also thank all members of the analytical laboratory, the mass spectrometry department and the NMR department for carrying out numerous measurements as well as all other employees of the institute.

I thank Kayla Friedman and Malcolm Morgan of the Centre for Sustainable Development, University of Cambridge, UK for producing the Microsoft Word thesis template used to produce this document.

I must give my deep thanks to Zhiyong. Through your patience, support and unwavering belief in me, I've been able to complete this dissertation journey. I did not realize how much he has done for me until you went back to China for his career. He has taken care of whatever needed tending to without complaining, just so I could focus on my research. There are no words that can express my gratitude for all you have done and been for me. Last but not the least, I would like to thank my parents for their faith in me, providing me with unending encouragement and support through all these years, and allowing me to be as ambitions as I wanted. I love them so much, and I would not have made it this far without them. I'd like to thank my beloved cats, Whisky and Oreo, for being so sweet all the time, for their companion and for sitting or sleeping closed to my laptop for hundreds of hours. 


\section{FULL LIST OF PUBLICATIONS}

\section{First-author publications}

"An Inclusion Complex of Hexamolybdate inside a Supramolecular Cage and its Structural Conversion", M. Han, J. Hey, W. Kawamura, D. Stalke, M. Shionoya, G. H. Clever, Inorg. Chem. 2012, 51, 9574. (27 citations)

Highlighted in the ACS Virtual Issue on "Synthetic Inorganic Chemistry" 2013 and in the ACS Inorganic Chemistry Virtual Issue on “Inorganic Cages and Containers" 2014.

“Light-triggered Guest Uptake and Release by a Photochromic Coordination Cage”, M. Han, R. Michel, B. He, Y.-S. Chen, D. Stalke, M. John, G. H. Clever, Angew. Chem. Int. Ed. 2013, 52, 1319. (108 citations, "Hot Paper")

“Rational Design of a Face-Centred Square-Cuboid Coordination Cage”, M. Han, R. Michel, G. H. Clever, Chem. Eur. J. 2014, 20, 10640. (8 citations)

Special Issue: European Young Chemists.

"Light-controlled Interconversion between a Self-assembled Triangle and a Rhombicuboctahedral Sphere”, M. Han, Y. Luo, B. Damaschke, L. Gómez, X. .Ribas, A. Jose, P. Peretzki, M. Seibt, G. H. Clever, Angew. Chem. Int. Ed. 2016, 55, 445. (6 citations, "VIP Paper").

\section{Review}

"Self-assembled Coordination Cages based on Banana-shaped Ligands", M. Han, D. M. Engelhard, G. H. Clever, Chem. Soc. Rev. 2014, 43, 1848. (95 citations) 


\section{CONTENTS}

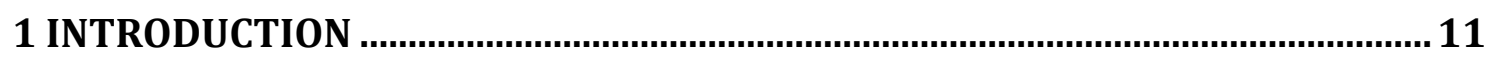

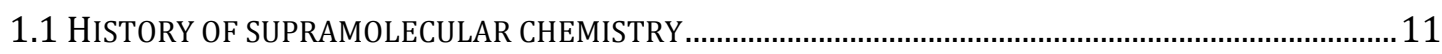

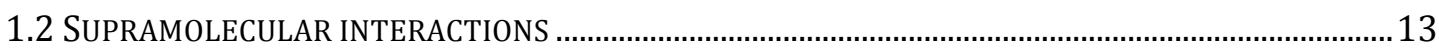

1.3 DESIGN PRINCIPLES OF TRANSITION METAL BASED SELF-ASSEMBLIES.............................................16

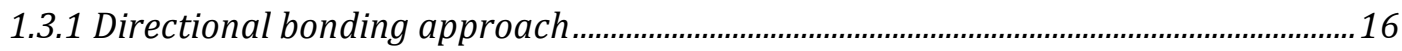

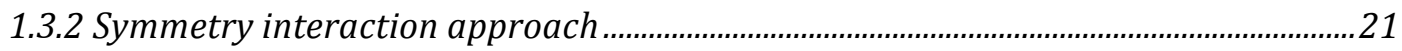

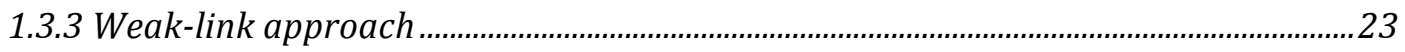

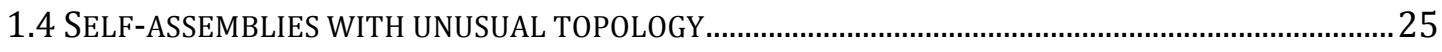

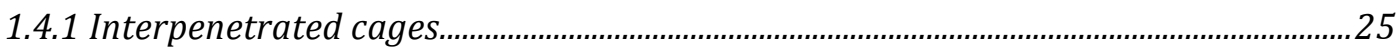

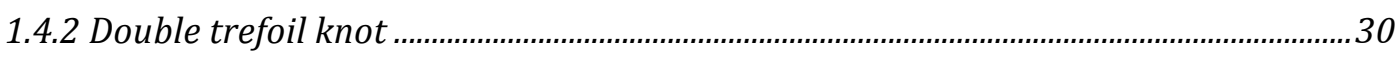

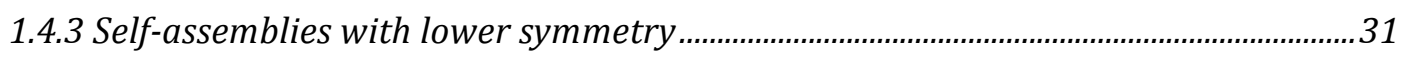

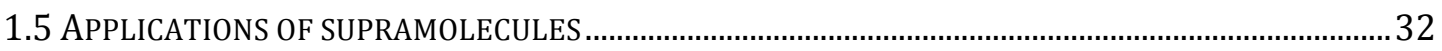

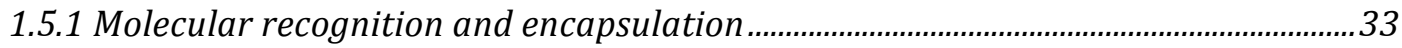

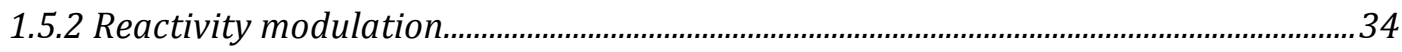

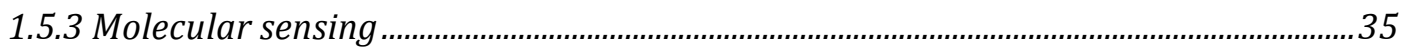

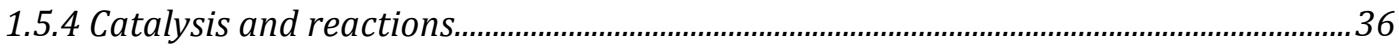

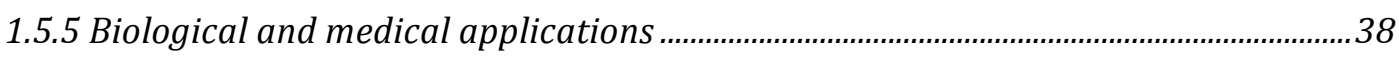

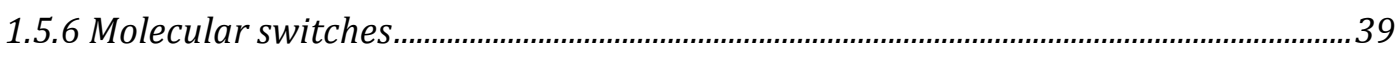

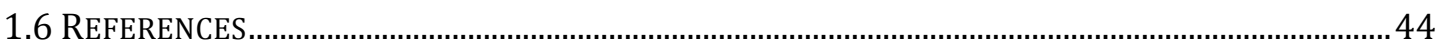

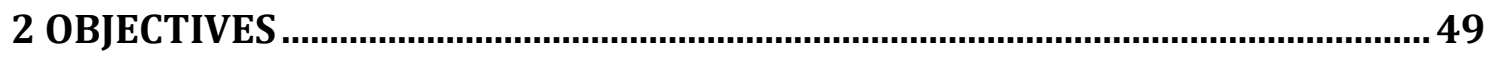

3 AN INCLUSION COMPLEX OF HEXAMOLYBDATE INSIDE A

SUPRAMOLECULAR CAGE.................................................................................. 53

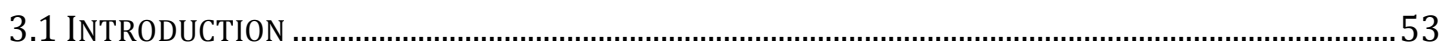

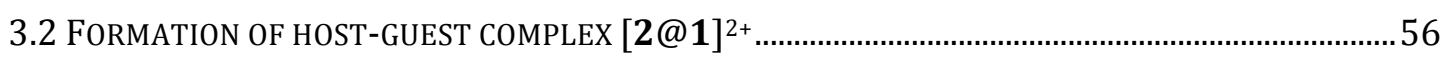

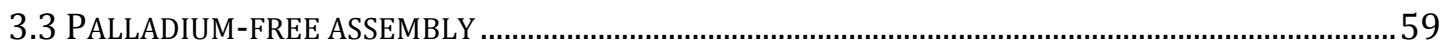

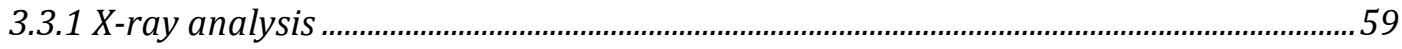

3.3.2 Quantum mechanical calculation of the C-F ‥Plane $e_{P y}$ interaction...............................62

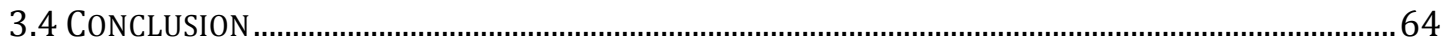

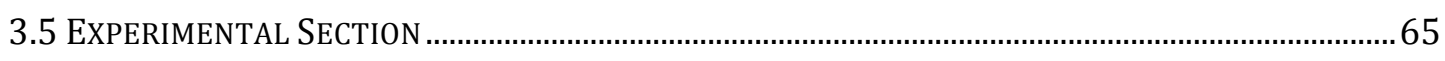

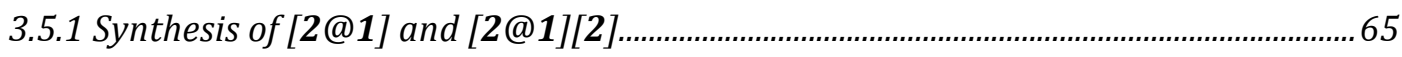

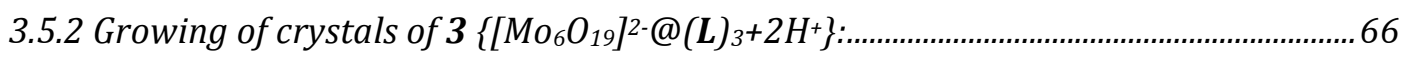

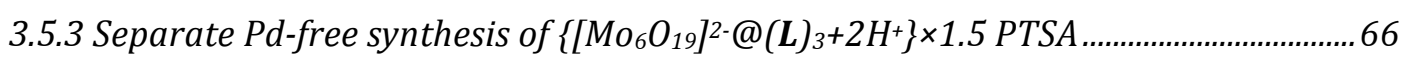

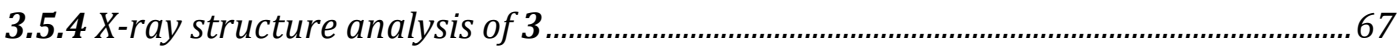

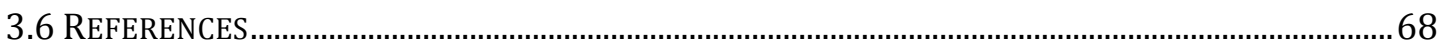

4 RATIONAL DESIGN OF A FACE-CENTRED SQUARE-CUBOID COORDINATION

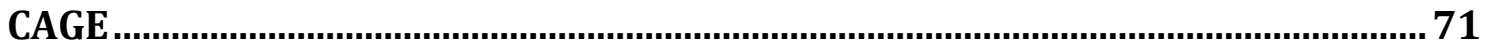




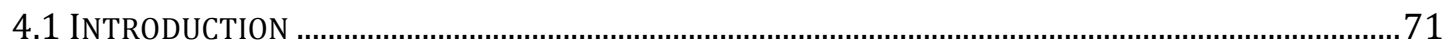

4.2 DERIVATION OF CAGE GEOMETRY AND MATHEMATICAL MODELLING ............................................73

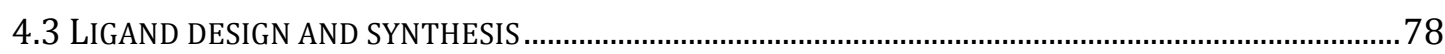

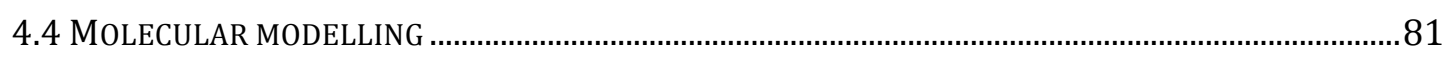

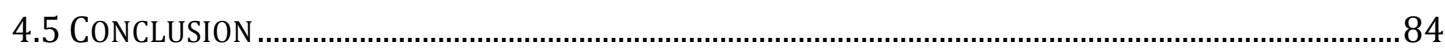

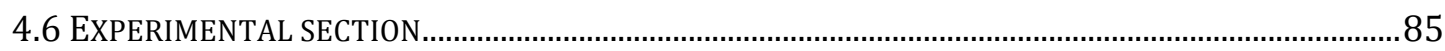

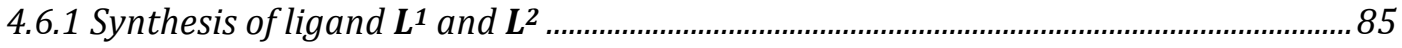

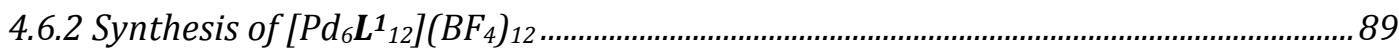

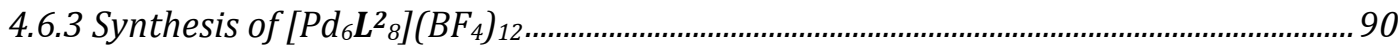

4.6.4 DOSY comparison of both cages............................................................................. 92

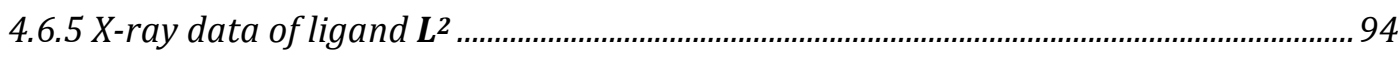

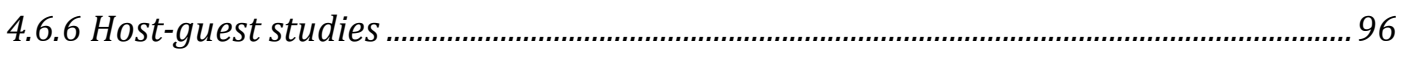

4.6.7 Molecular modelling results.............................................................................................. 97

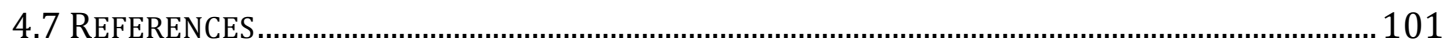

5 LIGHT-TRIGGERED GUEST UPTAKE AND RELEASE BY A PHOTOCHROMIC CAGE

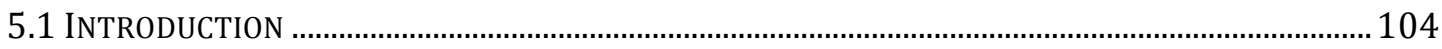

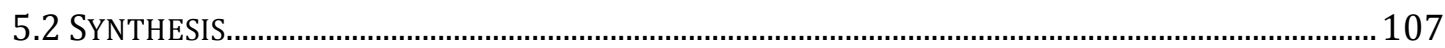

5.3 SYMMETRY INTERPRETATION .......................................................................................... 111

5.3.1 Symmetry-related interpretation of the NMR spectra ….............................................. 111

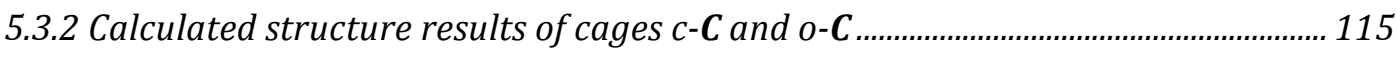

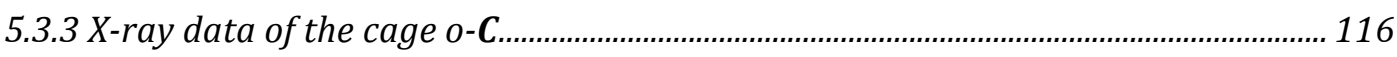

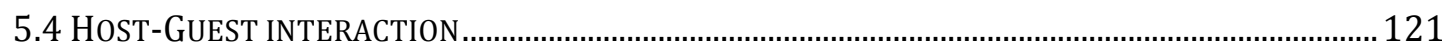

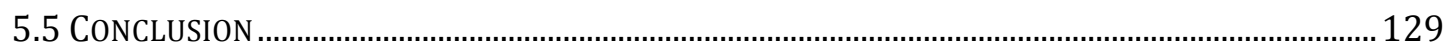

5.6 EXPERIMENTAL SECTION.............................................................................................. 130

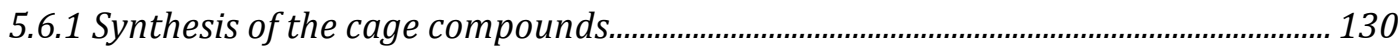

5.6.2 Temperature dependent titrations of o-C and $c$ - $\boldsymbol{C}$ with $\left[B_{12} F_{12}\right]^{2-}$ guest................. 132

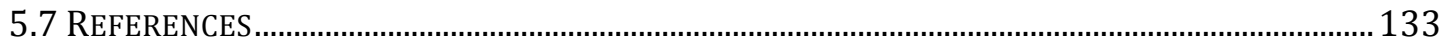

6 LIGHT-TRIGGERED CONVERSION BETWEEN A SELF-ASSEMBLED TRIANGLE

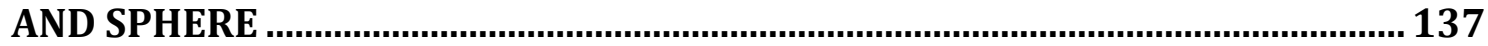

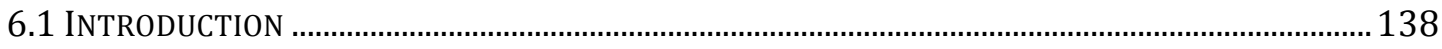

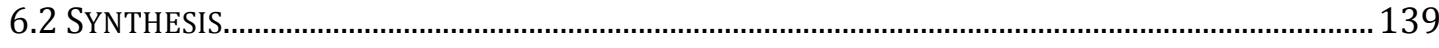

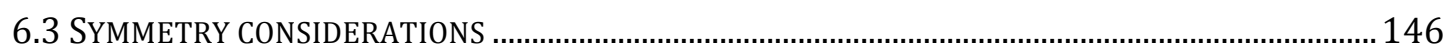

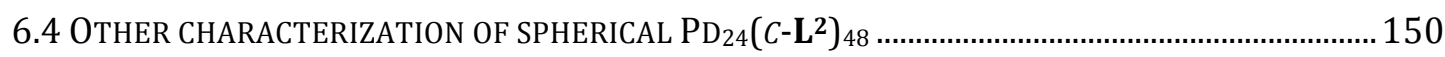

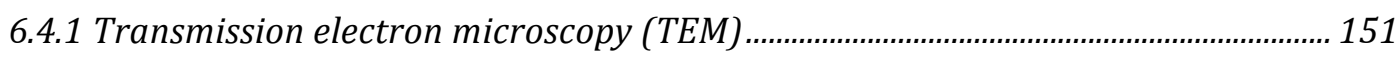

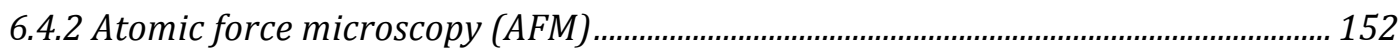

6.4.3 Grazing-incidence small-angle x-ray scattering (GISAXS)........................................ 153

6.5 KINETICS OF PHOTOCONVERSION …………………………...................................................... 154 


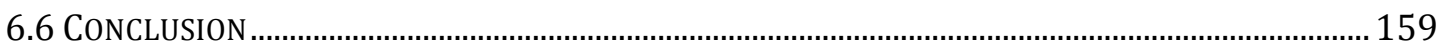

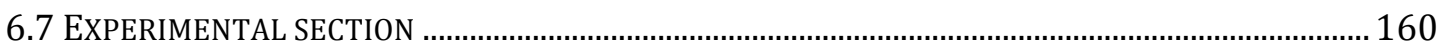

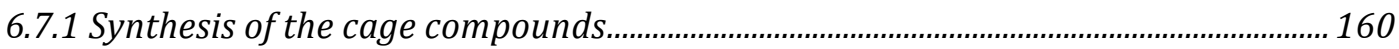

6.7.2 Synthesis of the three-membered ring with nitrate as counter anion....................... 162

6.7.3 Photoswitching between the ligand and ring/cage isomers .................................... 162

6.7.4 DOSY NMR spectra of the rings and cages................................................................... 163

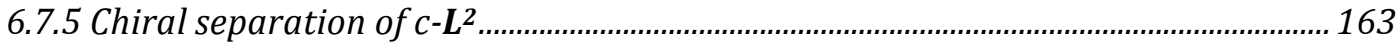

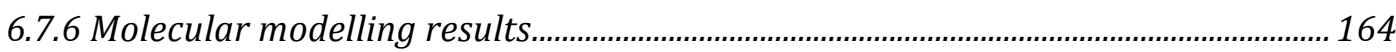

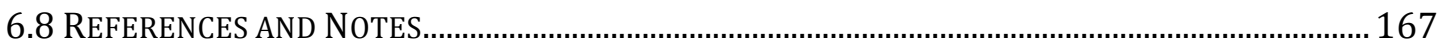

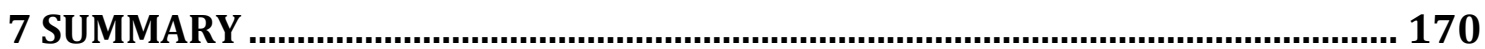

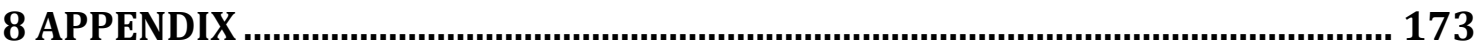




\section{INTRODUCTION}

\subsection{History of supramolecular chemistry}

Supramolecular chemistry was defined as the "chemistry of molecular assemblies and of the intermolecular bond".[1] Initially, supramolecular chemistry can be understood as describing the chemical species ("hosts" and "guests") that are held together and organized by intermolecular non-covalent interactions (Figure 1-1).[2]

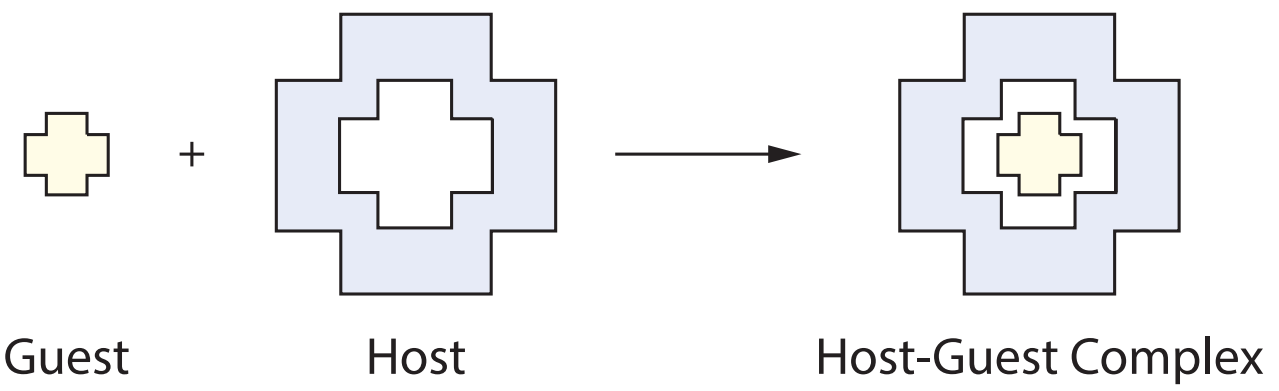

Figure 1-1 Scheme of supramolecular chemistry in respect of guest and host.

The beginning of supramolecular chemistry goes back to the late $19^{\text {th }}$ century, when the cyclodextrins, the first host molecules, were first introduced by A. Villiers. ${ }^{[3,4]}$ Shortly afterwards, Alfred Werner proposed the correct structures for coordination compounds containing complex ions, in which one central transition metal ( $\mathrm{Cu}, \mathrm{Co}, \mathrm{Pt}$, etc.) is surrounded by anionic or neutral ligands, and established the foundation for modern coordination chemistry.[5] In 1894 Emil Fischer described the "Lock and Key" model (Figure 1-2a), the basis of molecular recognition, according to which only the substrate has to possess specific shape that is complementary to the active site of one enzyme, to which it can bind and in whose pocket it can react.[6] This postulation was further refined 
by Daniel E. Koshland with the introduction of an "induced fit" model (Figure 1-2b).[7] In 1906 Paul Ehrlich introduced the concept of the "receptor", indicating that the molecules do not act if they do not bind ("Corpora non agunt nisi fixata").[8]

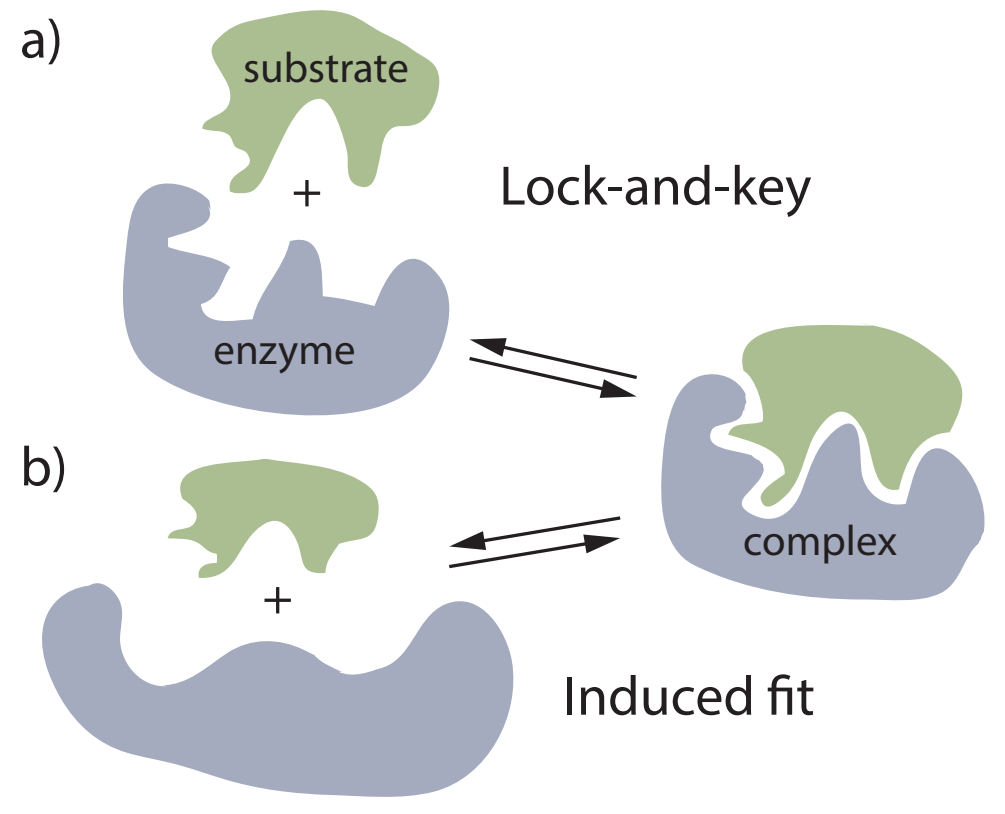

Figure 1-2 a) Lock and key and b) induced fit models of enzyme-substrate binding.

These concepts laid the foundation of supramolecular chemistry. This field was and still is considered as an interdisciplinary area, containing not only chemical, but also biologically derived species. Although the word "supramolecule" had already been introduced as "Übermolekül“ in the mid-1930s by Karl Lothar Wolf to describe hydrogen-bonded acetic acid dimers, ${ }^{[9]}$ it was a long journey until the supramolecular chemistry field started to expand rapidly, held back due to the limitations in the development of analytical methodology.

In 1967, a ground-breaking paper of Charles Pedersen was published, in which crown ethers (cyclic polyethers, containing $-\mathrm{OCH}_{2} \mathrm{CH}_{2}$ - repeating motif) were found in the course of the synthesis of acyclic polyethers (Figure 1-3a).[10] These types of compounds showed an attractive, unexpected and diverse ability to bind alkali and alkaline earth metals, such as lithium, sodium, potassium, rubidium and caesium. Soon after that, Jean-Marie Lehn extended the two-dimensional crown ethers into three-dimensional cryptands, ${ }^{[11]}$ which show even higher cation binding selectivity and stronger affinity, because of the increasing pre-organization of the binding-sites (Figure 1-3b). The spherands, designed and first studied by Donald J. Cram in 1979[12], are macrocyclic systems that are rigid and preorganized for binding to different cationic guests in solution (Figure 1-3c). The alkali 
metal binding ability of spherands is far stronger than that of the crown ether or cryptand hosts.

a)<smiles>CC1(C)OCCOc2ccccc2O1</smiles>

b)

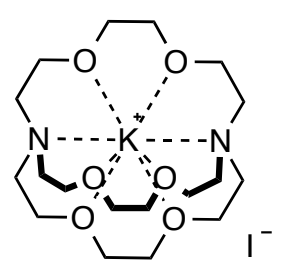

c)

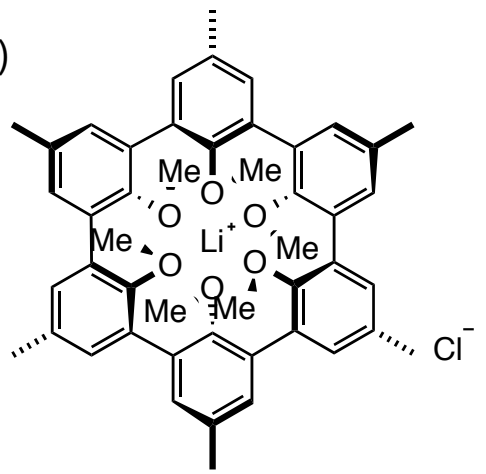

Figure 1-3 a) Dibenzo-[18]crown-6 complex (Pederson), b) [2.2.2]cryptand complex (Lehn) and c) spherand-6 complex (Cram).

The year 1987 is considered as a landmark in the history of supramolecular chemistry as Charles J. Pedersen, Jean-Marie Lehn and Donald J. Cram shared the Nobel Prize in Chemistry "for their development and use of molecules with structure-specific interactions of high selectivity".

In nature, there are plenty of examples containing supramolecular systems. One of the most prominent examples is the deoxyribonucleic acid (DNA) double helical structure discovered by James D. Watson and Francis H. C. Crick in 1953.[13] Two complementary phosphate-deoxyribose backbones are bound together by hydrogen bonding and $\pi$ stacking between base pairs in a helical fashion. Since then, chemists dedicate themselves to designing and developing supramolecular species, inspired by nature.

\subsection{Supramolecular interactions}

As described earlier, supramolecules are chemical species held together by non-covalent interactions, ${ }^{[1]}$ including hydrogen bonding, $\pi-\pi$ interactions, van der Waals forces. These non-covalent interactions are kinetically labile and weaker than covalent interactions. Non-covalent interactions can vary between $2 \mathrm{~kJ} \cdot \mathrm{mol}^{-1}$ for dispersion interactions to $300 \mathrm{~kJ} \cdot \mathrm{mol}^{-1}$ for ion-ion interactions. Typical strengths of non-covalent interactions are listed in Table 1-1.[14] 
Table 1-1 Summary of different non-covalent interactions.[14]

\begin{tabular}{|c|c|}
\hline Interaction & Energy values [kJ·mol-1] \\
\hline ion-ion & $200-300$ \\
\hline ion-dipole & $50-200$ \\
\hline dipole-dipole & $5-50$ \\
\hline hydrogen bonding & $4-120$ \\
\hline cation- $\pi$ & $5-80$ \\
\hline$\pi-\pi$ & $0-50$ \\
\hline van der Waals & $<5$, variable depending on surface area \\
\hline hydrophobic & depends on solvent-solvent interactions \\
\hline
\end{tabular}

The ion-ion interaction is the strongest of these non-covalent interactions, which can adopt similar values to covalent ones, and occurs between oppositely charged species. In nature, this ion-ion interaction can be present in any orientation. Thus, it is completely omnidirectional. The ion-dipole (Figure 1-3) and dipole-dipole interactions (Figure 1-4) are weaker compared to ion-ion, which require particular orientations of aligned species. Electrostatic interactions are considered as strong non-covalent bonds and play a crucial role in binding and recognition processes.
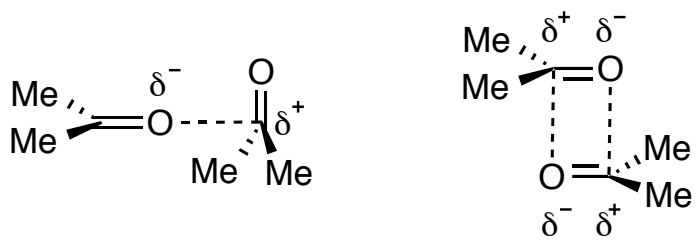

Figure 1-4 Dipole-dipole interactions of acetone molecules.

Hydrogen bonding is one special type of dipole-dipole interaction between an electronegative atom (H-bond acceptor $\mathbf{A}$ ) and a hydrogen atom bound to another electronegative atom (H-bond donor D). In many naturally occurring building blocks, hydrogen bonding plays a very important role, e.g. base-pairing in DNA double strands. The binding strengths are determined not only with respect to the type of electronegative atom attached to the hydrogen, but also by the geometry and number of hydrogen bond donors/acceptors (Figure 1-5). 
a)

$\mathbf{D}-\mathrm{H}-\mathbf{- A}$

d)<smiles>[CH]1COP1</smiles>

b)<smiles>[2H][13CH3]</smiles>

e)<smiles>[2H][13C]([2H])([2H])[13CH3]</smiles>

c)<smiles>[2H][13CH][13CH]</smiles>

f)<smiles>[3H]C1O[14C]1C</smiles>

Figure 1-5 Different types of hydrogen bonding geometries.[15]

Another important non-covalent interaction is $\pi$-interaction, which includes cation- $\pi$, anion- $\pi$ and $\pi-\pi$ interactions. "Face-to-face" and "edge-to-face" are the two general types of $\pi$ - $\pi$ interactions (Figure 1-6). "Face-to-face" $\pi$-interactions are observed between basepairs in DNA along the double helix.[16] The planar, layered structure of graphite represent another prominent example that is also held together by such "face-to-face" $\pi$-interactions. "Edge-to-face" interactions are responsible for the herringbone packing in the X-ray crystal structure of benzene molecules.

a)

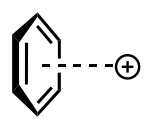

b)

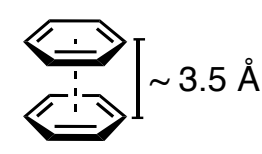

c)

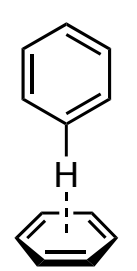

Figure 1-6 a) Cation- $\pi$ interaction and two types of $\pi-\pi$ interactions b) "face-to-face" and c) "edge-to-face" manner.

The van der Waals force is much weaker in comparison with the other non-covalent interactions discussed above. They are composed of dispersion and exchange-repulsion between molecules,[15] and can be applied generally in all kinds of molecules.

On the weak end of non-covalent interactions, the hydrophobic effect plays an essential role in guest binding and recognition of supramolecular systems in polar media, for example the guest binding by cyclodextrins in water. The solvent molecules, which reside inside the cavity of the host, cannot interact with the host strongly, because their interactions with other solvent molecules outside the cavity are significantly stronger. This means, the solvent molecules can be easily replaced by guests with similar polarity 
with respect to the host. Upon release of the solvent molecules, the energy of the system is lowered by the interaction of former solvent guest molecules with the bulk solvent out of the host and the ordered solvent molecules within the cavity become disordered when they were replaced. Once the guest was encapsulated inside the host cavity, the formation of host-guest complex results in less disordering to the solvent structure and resulting in an increase in entropy (Figure 1-7).

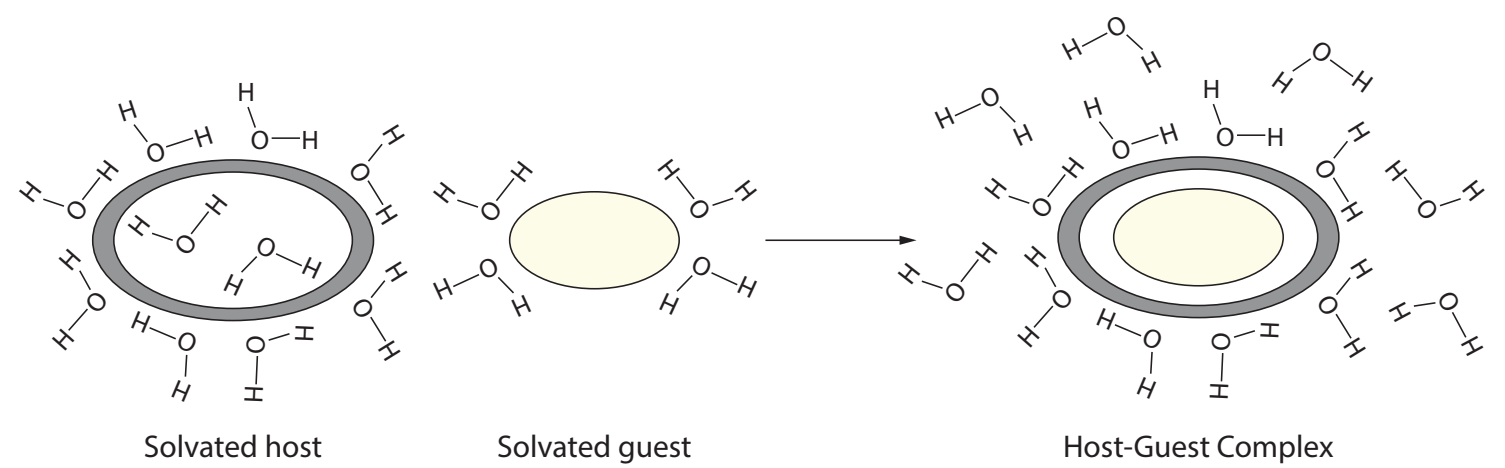

Figure 1-7 Scheme of hydrophobic effect of organic guest binding.[15]

\subsection{Design principles of transition metal based self-assemblies}

In transition metal based self-assembled systems, ligand precursors are held together by metal-ligand coordinative bonds. The metal ions and ligands, assemble selectively to form large aggregates and usually generate the thermodynamically favoured supramolecular structure. The physical and chemical properties of the self-assembled aggregates are normally different from their parent building blocks. Self-repair and correction are essential features in the synthetic strategy and are a key advantage of non-covalent chemistry.[17] Over the last decades, supramolecular chemists have created a large number of two- and three-dimensional structures, such as squares,[18] hexagons,[19] grids,[20] helicates,[21] nanocapsules[22] and cages.[23] As the shape of the self-assembled molecules can be predetermined depending on the coordination geometry of the metal ions and the orientation of the binding sites of ligands, it is possible to design and predict the resulting supramolecular structures by using simple geometrical considerations and synthetic strategies. Three synthetic design strategies[24] have emerged subsequently: the directional bonding,[25] the symmetry interaction[26] and the weak-link approach.[27]

\subsubsection{Directional bonding approach}

The directional bonding approach is considered to be a general, high-yielding synthetic strategy for constructing a variety of supramolecular assemblies. This approach was 
pioneered by Verkade and co-workers in 1983.[28] Mixing of a diphosphine bridging ligand $\mathrm{P}\left(\mathrm{OCH}_{2}\right)_{3} \mathrm{P}$ with metal carbonyl precursors generated homo- and heteronuclear squares (Figure 1-8).
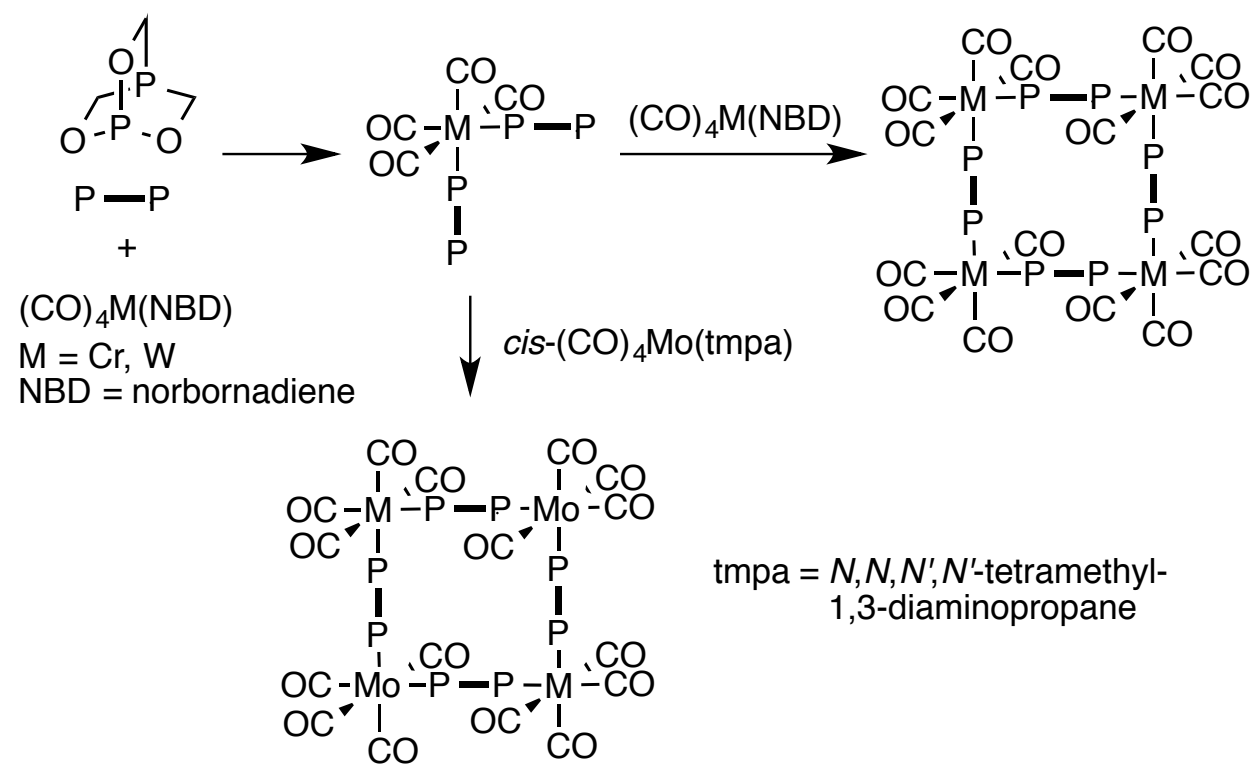

Figure 1-8 Direct coordination driven tetranuclear self-assembly.[28]

In 1990, Fujita et al. developed a quantitative synthesis of a molecular square by introducing a protected Pd(II) fragment and a 4,4'-bipyridyl ligand (Figure 1-9).[29] The fundamental principles were consequently systematized and formulated for the first time by Stang and named "molecular library model".[19,25] Since then, this strategy has been intensively studied by Fujita,[30] Mirkin,[24] and Swiegers.[31]

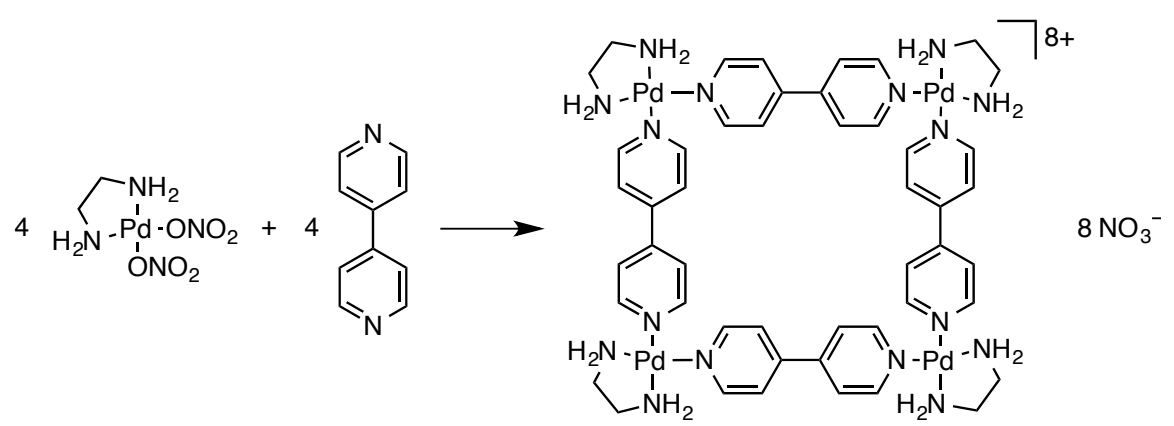

Figure 1-9 Cis-protected palladium-based molecular square.[29]

In general, the transition metal-containing subunits are considered as acceptors, because of the specific coordination sites and geometries of the metals. These metals can be connected with donor organic subunits and build rigid assembled structures. All of the subunits are supposed to own specific geometries and to be multidentate, which means 
each subunit should possess at least two sites available for coordination. The angle between the binding sites of organic donor ligands ranges from $0^{\circ}$ to $180^{\circ}$. When the subunits with predefined binding angles are linked together in appropriate stoichiometric ratio, the resulting species depends on the number and geometry of binding sites of each subunit. The design principle of two-dimensional structures is illustrated in Figure 1-10. ${ }^{[32]}$

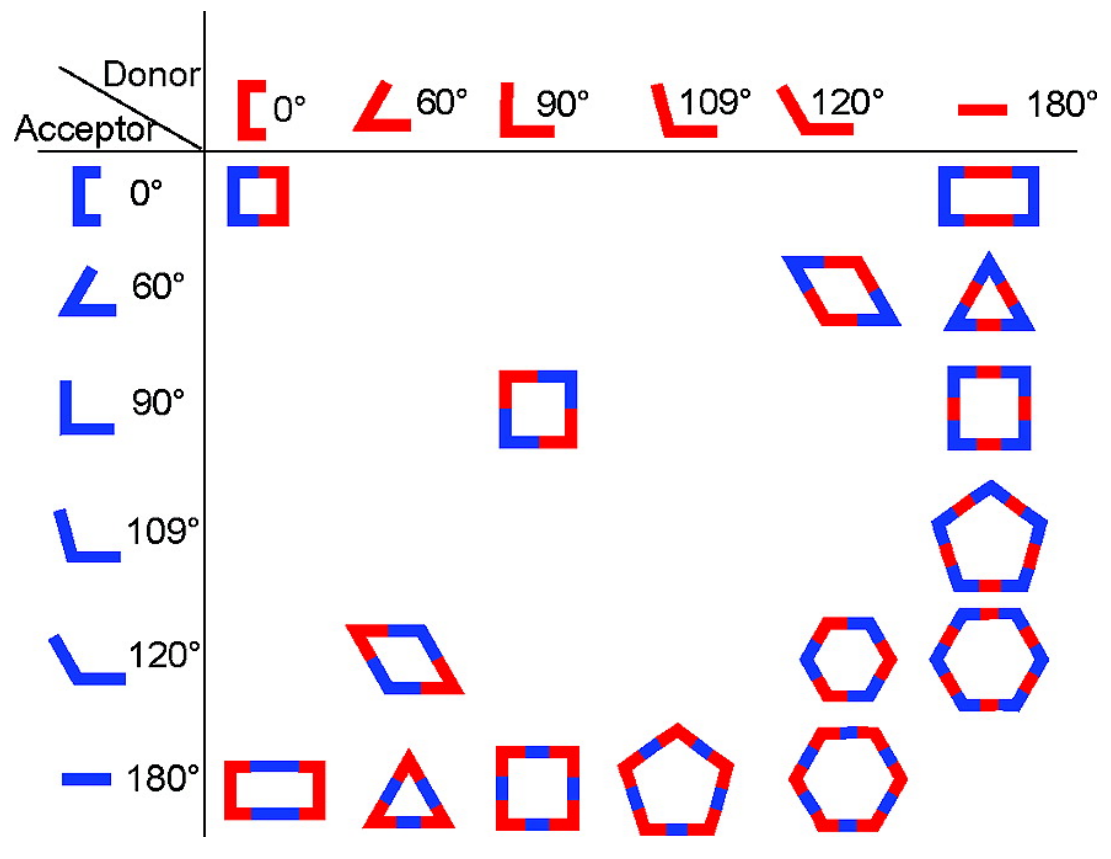

Figure 1-10 Formation of two-dimensional macrocycles using directional bonding approach.[32] Copyright (C) 2011 American Chemical Society.

Among the two-dimensional macrocycles, tetranuclear squares represent the most frequently reported structures. An interesting porphyrin square was reported by Drain and Lehn (Figure 1-11a).[33] 5,10-Pyridylporphyrin units have been used as angular units, of which the pyridyl groups are orientated in $90^{\circ}$ angle to each other, and linked by trans$\left[\mathrm{PdCl}_{2}\right]^{2+}$ metal centres as linear bridging units. An interesting complex has been synthesized by Würthner et al. by combining ditopic perylene bispyridylimide ligands and $\mathrm{Pd}^{\mathrm{II}}$ or PtII phosphine corner units. The pyridyl perylene units conserved their optical and electrical properties after the formation of the complex (Figure 1-11b).[34] The perylene based metallocycle was found to possess excellent luminescence properties as well as redox activity after functionalization of the backbone by introduction of a ferrocene unit (in Figure 1-11b, $\mathbf{R}_{\mathbf{2}}$ as substituent).[35] 
a)

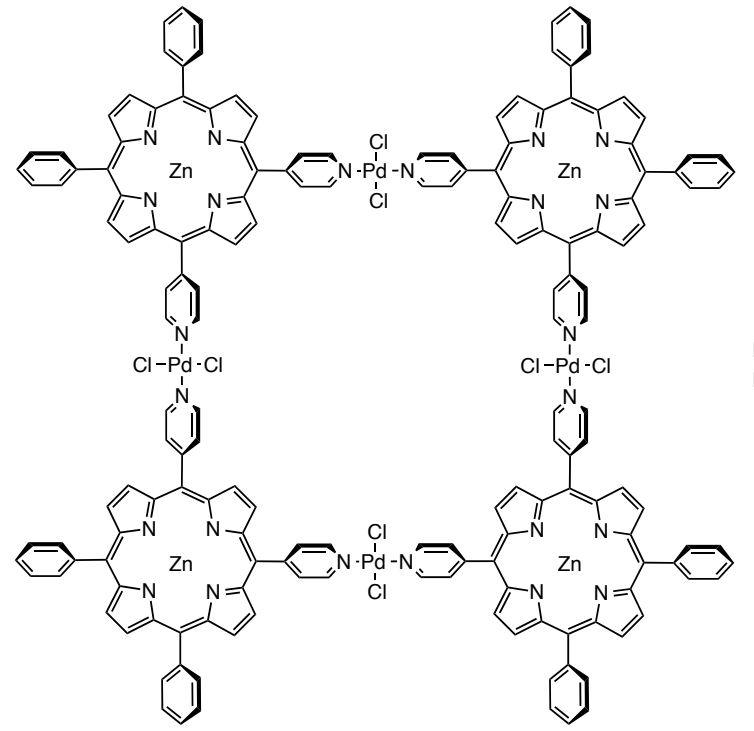

b)

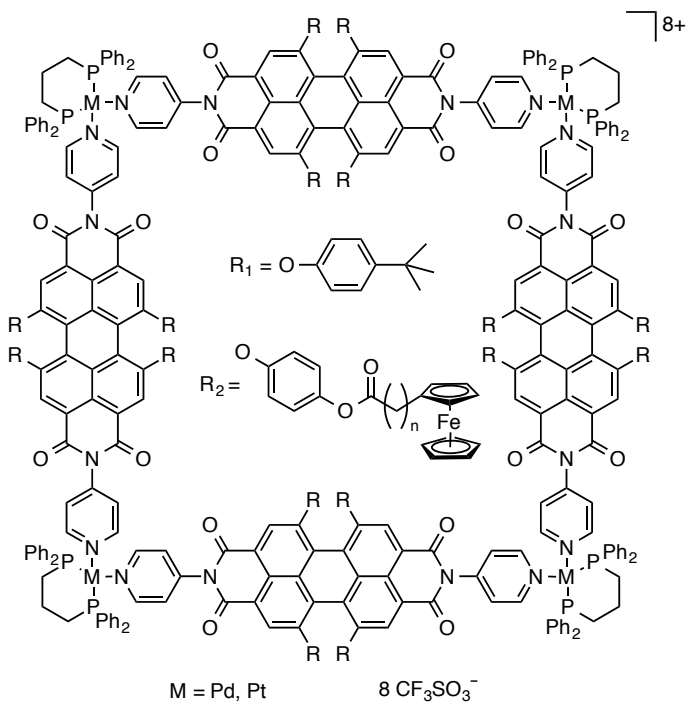

Figure 1-11 a) Porphyrin ${ }^{[33]}$ and b) perylene ${ }^{[34]}$ involving self-assembled square tetramers.

The design of three-dimensional polyhedra is significantly more complicated, as a larger number of building blocks have to be involved for their construction. At least one of the building blocks should possess three active binding sites in order to construct a threedimensional structure (Figure 1-12).

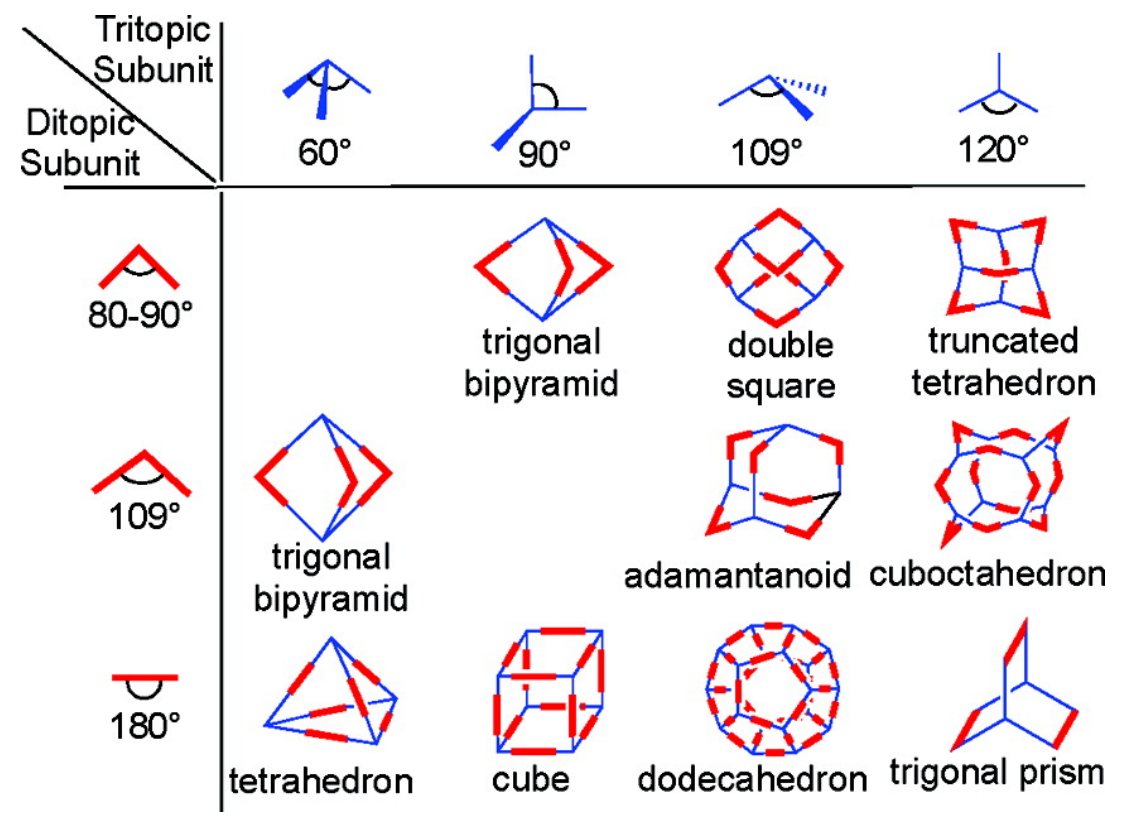

Figure 1-12 Accessible three-dimensional polyhedra combining ditopic and tritopic building blocks.[32] Copyright (C) 2011 American Chemical Society. 
The formation of self-assembled polyhedra via the directional bonding approach can be either edge- or face-directed. In the edge-directed strategy, all building blocks lie on the edge of the generated polyhedra, whereas in the face-directed approach, the faces of the target molecule are covered by the building units (Figure 1-13).[36]

a)

8

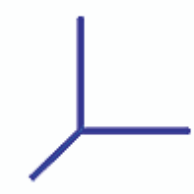

b)

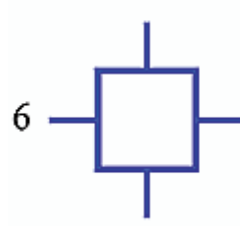

$+\quad 12$

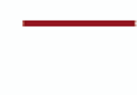

$+$

12

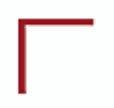

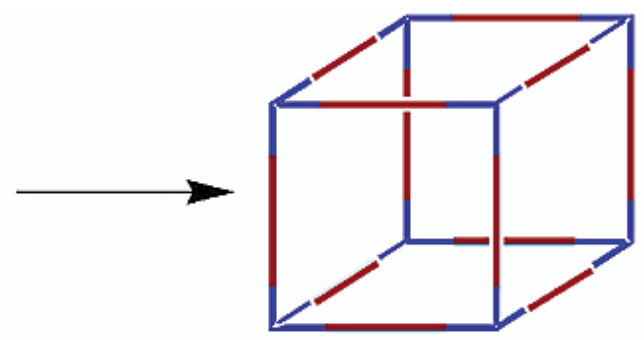

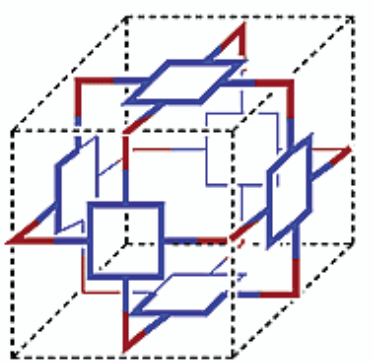

Figure 1-13 a) Edge- and b) face-directed self-assembly.[36] Copyright (C) 2002 American Chemical Society.

For example, a supramolecular cube could be obtained by mixing 8 tritopic corner units with $90^{\circ}$ angles and 12 ditopic linear linkers using the edge-directed technique $\left(\mathrm{M}_{8} \mathrm{~L}_{12}\right.$ in Figure 1-14a).[37] Moreover, a cube could be synthesized using the face-directed method via the combination of 6 tetratopic planar faces and 12 ditopic $90^{\circ}$ building blocks with a different ligand to metal ratio $\left(M_{12} \mathbf{L}_{6}\right.$ in Figure 1-14b).[38] Both strategies have been extensively applied by several research groups, and allowed to the formation of most of the Platonic and Archimedean solids.[32] 
a)

8
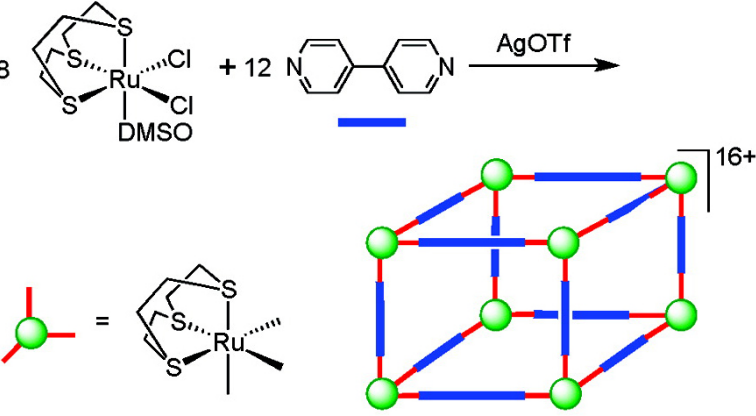

b)

12
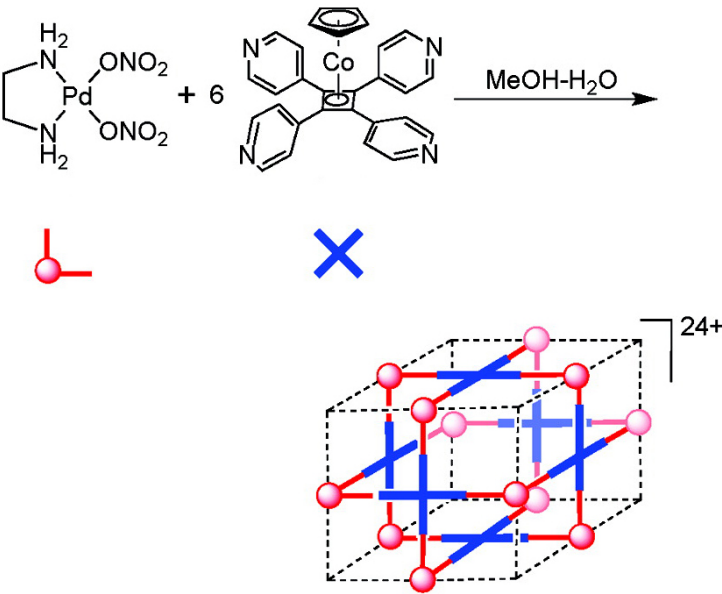

Figure 1-14 Example of a) edge- and b) face-directed supramolecular cube.[32] Copyright (C) 2011 American Chemical Society.

\subsubsection{Symmetry interaction approach}

The symmetry interaction approach is a high-yield rational design strategy for the synthesis of highly symmetric coordination clusters. Chelating ligands are used to coordinate transition or main group metal ions based on their geometric relationship. The driving force of the coordination procedure originates from the strong binding affinity of chelating ligands and the symmetry of the coordination sites of the metal centre. The metal-ligand combinations should be carefully chosen. The orientation of the multiple binding sites of the ligands must be taken into consideration in order to avoid the formation of oligomers and polymers. The symmetry interaction approach relies on thermodynamic control, as for the directional binding approach.[24,32]

The first compound synthesized by this strategy was introduced by Maverick in the mid 80s (Figure 1-15).[39,40] Using this approach, a substantial amount of work has been accomplished, especially by the groups of Saalfrank,,[41-43] Lehn,[44] and Raymond.[26] 

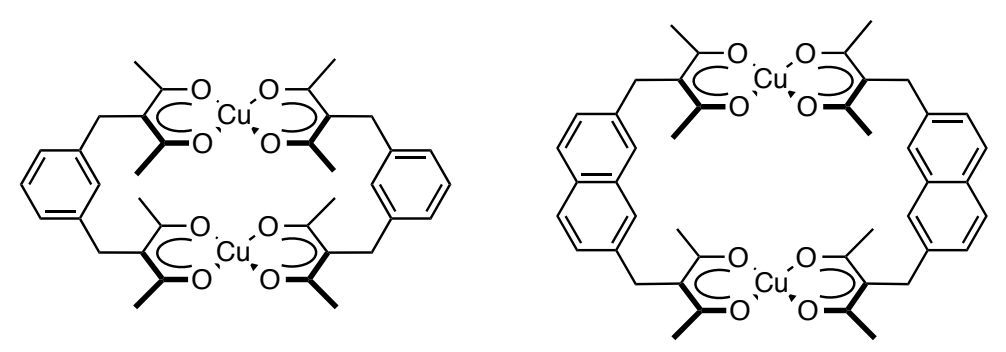

Figure 1-15 First metallomacrocyle employing the symmetry interaction approach.

In order to describe the symmetric interaction approach more accurately, Caulder and Raymond have defined several terms for the precise description of the relevant geometric relationships. ${ }^{[26,45]}$ First to mention is the coordinate vector, which is the vector from the coordinating atom of ligand to the metal centre (Figure 1-16a). Next, chelate plane is defined as the plane perpendicular to the major symmetry axis of the metal complex. All the coordinate vectors between the chelate ligands and metal centre lie in the chelate plane (Figure 1-16b). The last term that was introduced is the approach angle, which is defined by the angle between the two coordinating atoms of the chelating ligand and the major symmetry axis of the metal complex (Figure 1-16c). In principle, by using these considerations, it is possible to construct supramolecular structures with any symmetry.

a)

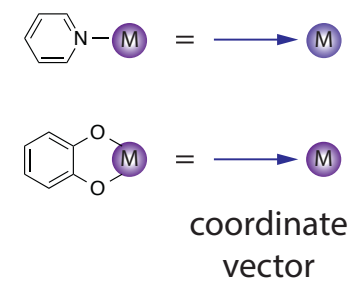

b)

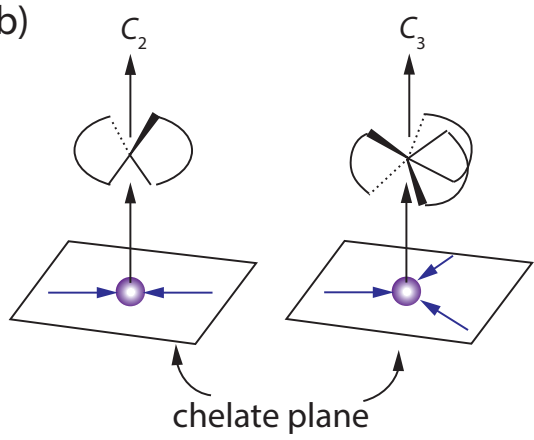

c)

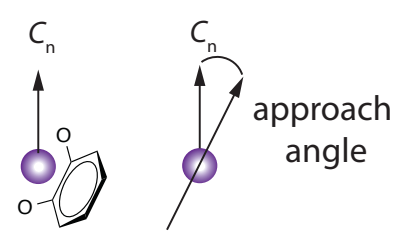

Figure 1-16 Models represent the definition of the symmetry interaction approach. a) Coordinate vector, b) chelate plane and c) approach angle.

Raymond and co-workers introduced a special class of complexes, which were attained by the symmetry interaction approach. ${ }^{[46,47]} \mathrm{An}\left[\mathrm{M}_{4} \mathbf{1}_{6}\right]$ cluster $\mathbf{2}$ with an overall -12 charge was synthesized by utilizing different metal salts $\left(\mathrm{Ga}^{3+}, \mathrm{Fe}^{3+}, \mathrm{Al}^{3+}\right)$ with bis(bidentate) ligand 1, which contains a naphthalene spacer and two catecholamides as binding units. Each metal ion locates at one of the four vertices of the tetrahedron, and linked by six bifunctional ligands. Each ligand represents one of the six edges of the tetrahedron (Figure 1-17). This tetrahedral anionic capsule is water-soluble and its voluminous hydrophobic cavity could encapsulate monocationic guests such as ammonium and 
ferrocenium ions. It performs as supramolecular catalyst in the 3-aza-Cope rearrangement ${ }^{[48]}$ and for the hydrolysis of orthoformates.[49]

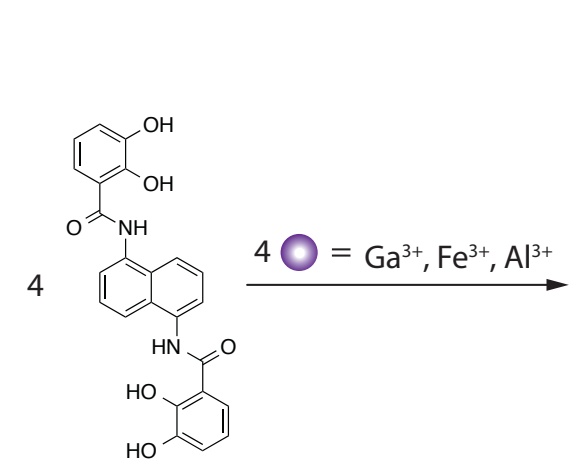

1

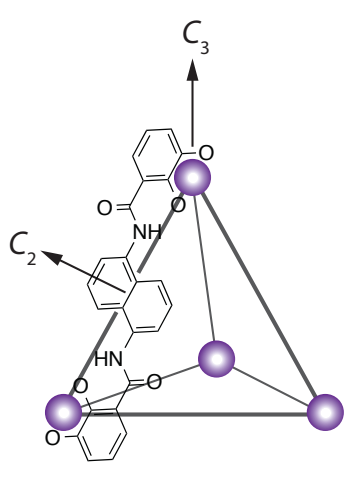

2

Figure 1-17 $\left[\mathrm{M}_{4} \mathbf{1}_{6}\right]$ tetrahedral coordination complex 2.

\subsubsection{Weak-link approach}

Both directional bonding and symmetry interaction approach rely on rigid ligands with well-defined coordination groups and metal ions with suitable binding sites. Using these approaches, highly symmetric self-assemblies with specific chemical reactivity, such as molecular recognition [50-52] and specific catalytic activity, can be generated.[49,53-55]

Mirkin and co-workers developed a different methodology by using more flexible ligands and metal centres with free coordination sites.[56] Flexible hemilabile ligands ${ }^{[57,58]}$ were used. They are asymmetric chelate ligands containing two different binding moieties, which have different binding affinities to the metal centre. When these hemilabile ligands were treated with metal ions, a kinetically controlled intermediate quantitatively assembled. The resulting intermediate was driven by the chelating effect of the bidentate ligands and the existing $\pi-\pi$ interaction between the central bridging units (Figure 1-18a). The weaker ligand-metal interactions can be broken, by exposing the condensed structure to small ligands or ions (ancillary ligands) with stronger affinity to the metal centres. Hence, the condensed macrocycle expands and forms the thermodynamic open product (Figure 1-18b). By the cleavage of the ancillary ligands, the condensed intermediate could be obtained again. 


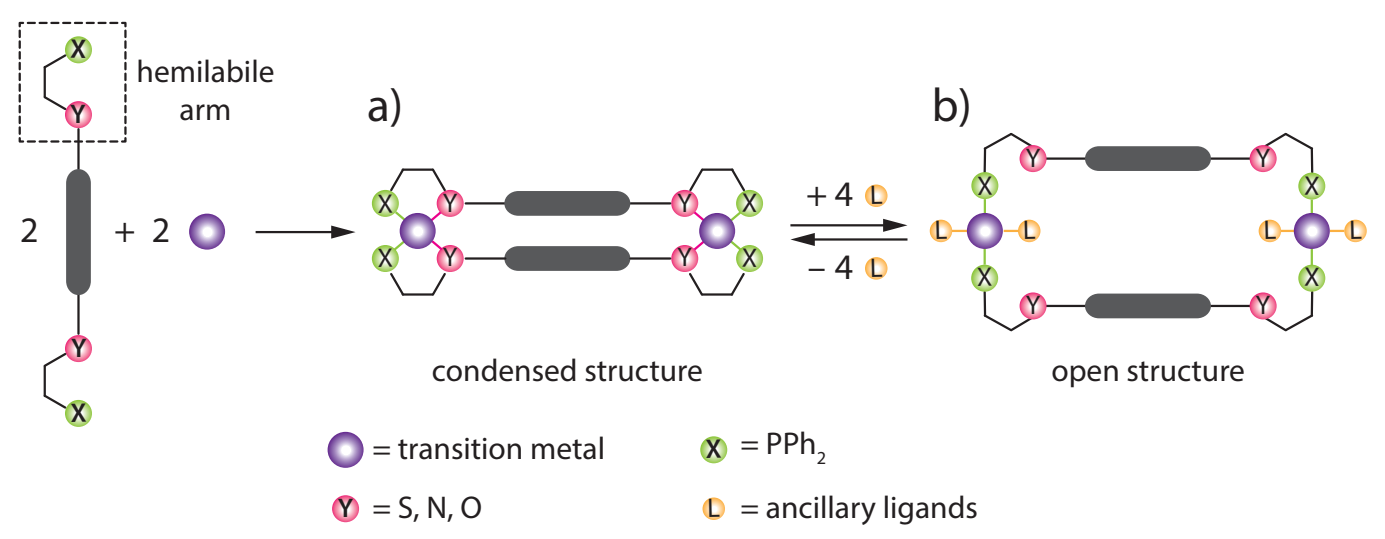

Figure 1-18 General principle of the weak-link approach.

The conformational flexibility of the metal-linking ligand is one of the key features of this approach, since it initiates the expansion from the condensed to a flexible open structure. It further provides the possibility to construct larger structures and networks.
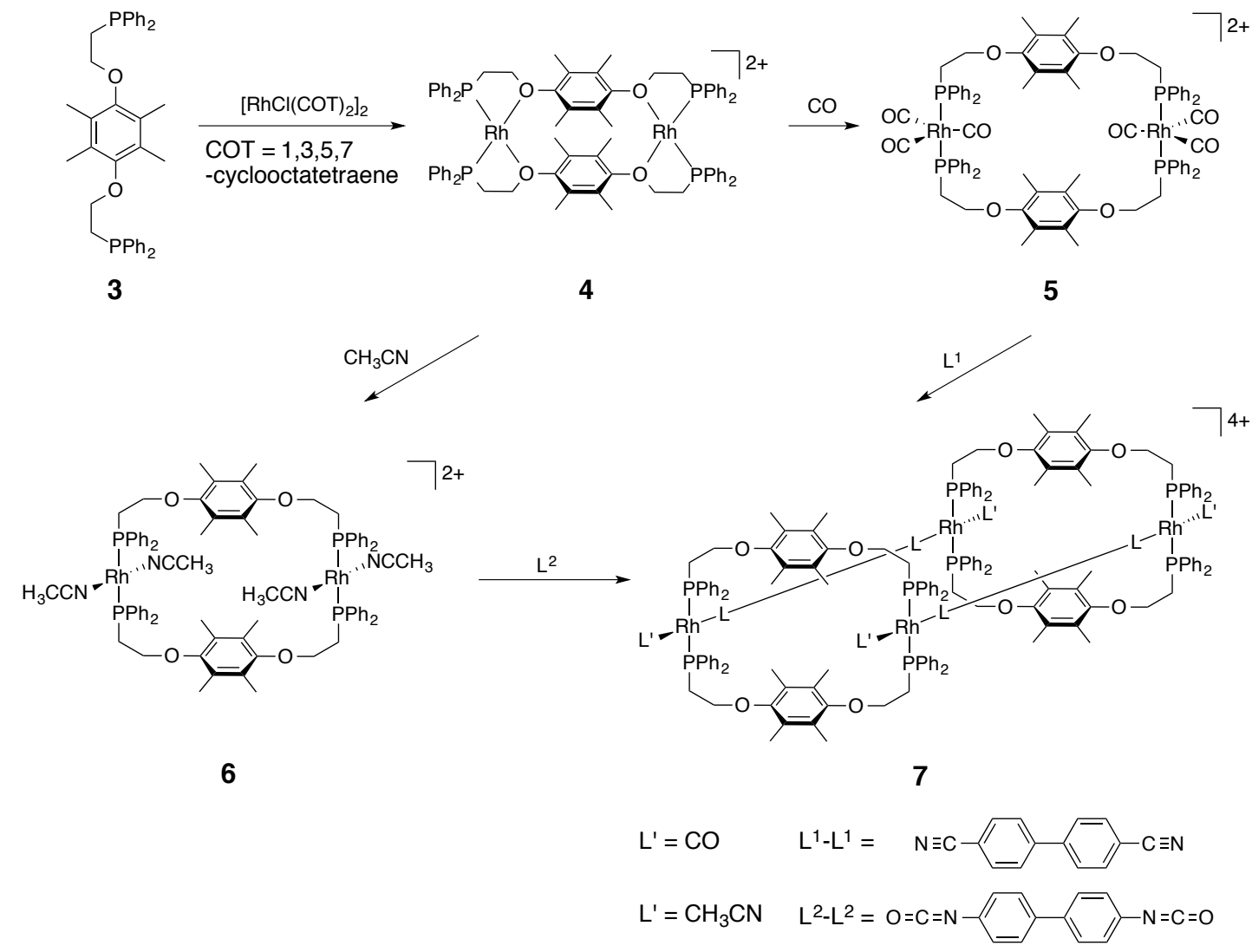

Figure 1-19 Synthesis of 3D cylinders 7 by extending 2D flexible macrocyclic structures 5 and 6 employing the weak-link approach. ${ }^{[59]}$

Pioneering work in this field has been performed by Mirkin and co-workers, who used flexible hemilabile ligand 3 and a square-planar $\mathrm{Rh}^{\mathrm{I}}$ precursor to form the condensed macrocycle 4. As soon as the ancillary ligands $\mathbf{L}^{\prime}\left(\mathrm{CO}\right.$ or $\left.\mathrm{CH}_{3} \mathrm{CN}\right)$ were introduced, the 
flexible open intermediates 5 or $\mathbf{6}$ were observed, respectively. By connecting two flexible intermediates using rigid bidentate linker L-L such as 4,4'-biphenyldicarbonitrile or 4,4'biphenyldiisocyanide, tetranuclear three-dimensional molecular cylinders 7 could be subsequently prepared. It contains two linked macrocycles and capped by either CO or acetonitrile ligands. The weak-link approach further could be used to generate extended metal-organic framework (MOF) tubes, since each end of the cylinder could be cleaved and linked by addition of bridging ligands (Figure 1-19).[59]

\subsection{Self-assemblies with unusual topology}

At the beginning, research was mainly aimed at producing rather symmetric molecules, such as helicates,[21,60] grids,[20] rings and cages. ${ }^{[32]}$ Most of the cages reported to date have very high symmetries, often resembling the Platonic or Archimedean solids in shape. Recently, the realization of non-trivial topologies has gained a lot of attention.[61]

\subsubsection{Interpenetrated cages}

\subsubsection{Work from other groups}

Interpenetrated coordination cages are a special class of the supramolecular structures with non-trivial topology, in which two monomeric units are mechanically interlocked without direct bonding between each other. Fujita and co-workers reported the first example of coordination cages featuring an interpenetrated structure in 1999.[62] The cage $\mathbf{1 1}$ is composed of two different tridentate ligands $\mathbf{9}$ and $\mathbf{1 0}$ with cis-protected $\mathrm{Pd}^{\mathrm{II}}$ and $\mathrm{Pt} \mathrm{tI}^{\mathrm{II}}$ complexes 8 in a 1:1:3 stoichiometry in $D_{2} \mathrm{O}$ (Figure 1-20). The interlocked double cage is favoured over the monomeric cage because of the efficient $\pi$ - $\pi$ stacking between the aromatic ligands. The interpenetration results in an increase of entropy due to the release of solvent molecules and counteranions from the monomeric cage.

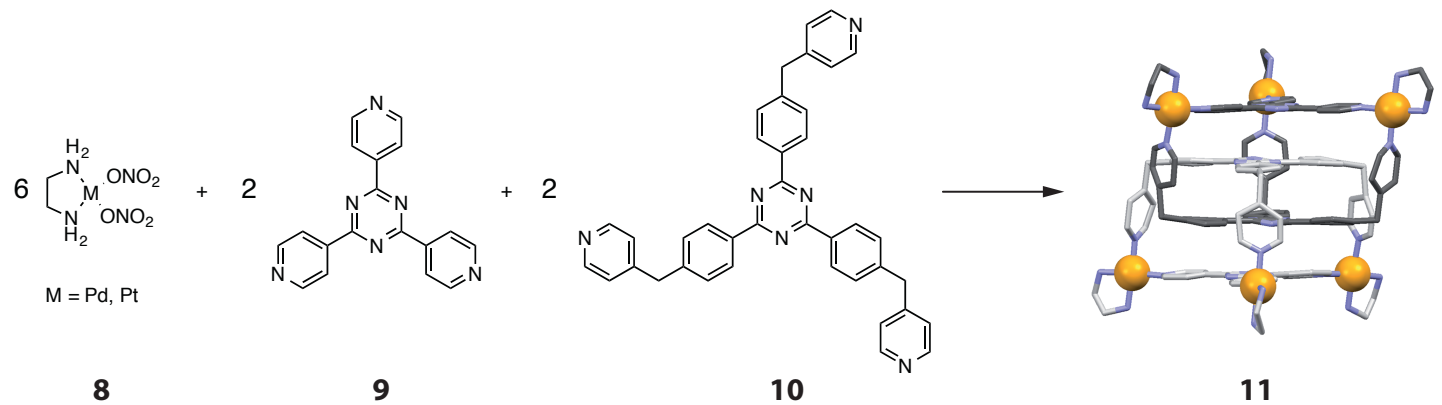

Figure 1-20 An interpenetrated double cage 11 constructed from cis-protected metal complexes $\mathbf{8}$ and different tridentate ligands $\mathbf{9 , 1 0}$. 
Another example of an interlocked double cage was prepared by Hardie and coworkers.[63] Treatment of $\mathrm{Zn}\left(\mathrm{NO}_{3}\right)_{2}$ or $\mathrm{Co}\left(\mathrm{NO}_{3}\right)_{2}$ with bowl-shaped cyclotrivertatrylenerelated ligand $\mathbf{1 2}$ in DMSO resulted in the formation of an interpenetrated double cage $\mathbf{1 3}$ (Figure 1-21). Two ligands coordinate to three equivalent metal centres and form a trigonal bipyramidal monomeric cage, in which the $\mathrm{Zn}^{\mathrm{II}}$ or $\mathrm{Co}^{\mathrm{II}}$ centres are in a slightly distorted octahedral geometry with one chelating nitrate anion and two chelating bipyridyl moieties from the two distinct ligands. The interpenetrated structure $\mathbf{1 3}$ of the two monomeric cages is a hydrogen bond-driven assembly, since the distance between the aromatic rings is too large (4.77 $\AA$ ) to form any $\pi$ - $\pi$ stacking interactions. In the following, they reported a similar triply interlocked double cage using pyridyl cyclotriguaiacylene ligand and linear coordinated $\mathrm{Ag}^{\mathrm{I}}$ metal centres with a $4: 6$ ratio.[64]

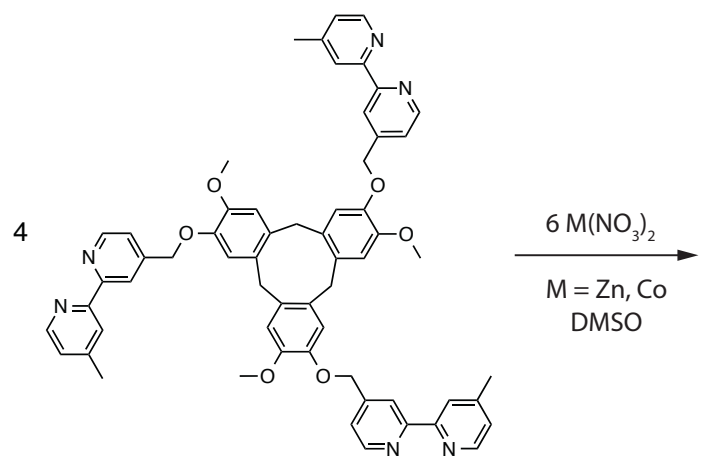

12

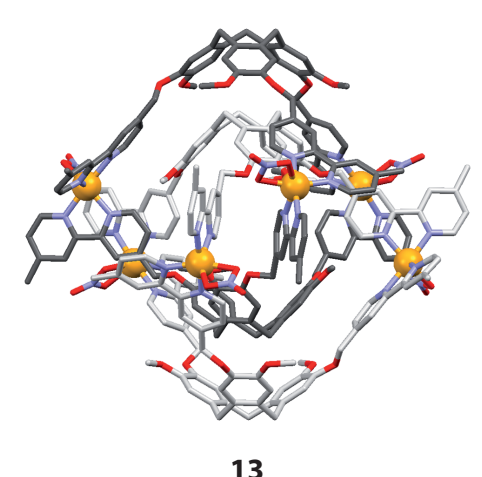

13

Figure 1-21 Synthesis of the cyclotriveratrylene-based interpenetrated double cage 13.

Kuroda and co-workers reported a dimeric interpenetrated double cage, which is composed of $\mathrm{Pd}\left(\mathrm{NO}_{3}\right)_{2}$ and flexible ligands based on benzophenone in a ration 4:8. Different from the previous interpenetrated cages, all the metal ions are located along one single $C_{4}$-axis, resulting in three separate cavities, in which counter anions or solvents could be possibly encapsulated.[141]

\subsubsection{Work from the Clever group}

The Clever group has synthesized a series of structurally related interpenetrated double cages and the binding of anions inside the double cages and the template effect were investigated in great detail.[61] The first double cage $\mathbf{1 5}$ of the Clever group was constructed from eight bis-monodentate pyridyl ligands 14 based on a dibenzosuberone backbone and four square-planar coordinating PdII cations.[65] Reaction of ligands 14 with $\left[\mathrm{Pd}\left(\mathrm{CH}_{3} \mathrm{CN}\right)_{4}\right]\left(\mathrm{BF}_{4}\right)_{2}$ formed initially the thermodynamically unstable monomeric $\left[\mathrm{Pd}_{2} \mathbf{1 4}_{4}\right]^{4+}$ cage, and yielded interpenetrated double cage 15 quantitatively after prolonged heating (Figure 1-22a). 
a)

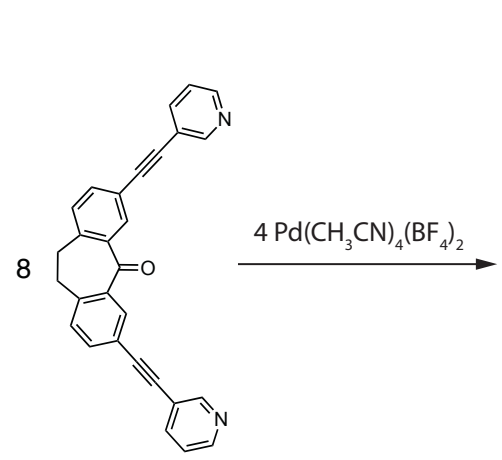

14

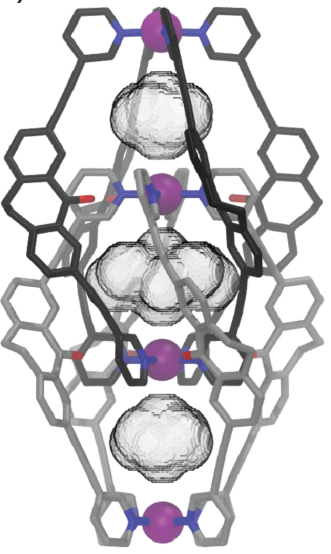

15 b)

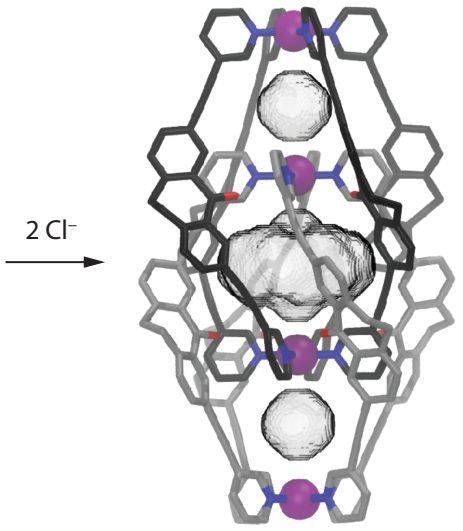

16

Figure 1-22 a) Synthesis and X-ray structure of interpenetrated double cage 15 containing three $\mathrm{BF}_{4}^{-}$anions in its central and outer pockets. b) DFT calculated structure of double cage $\mathbf{1 5}$ after the exchange of $\mathrm{BF}_{4}^{-}$from the outer pockets by $\mathrm{Cl}^{-}$anions (anions not shown). Copyright (c) 2014 The Royal Society of Chemistry.

Double cage 15 contains one central and two outer pockets, in which three guests could be bound. The central pocket binds $\mathrm{BF}_{4^{-}}$much tighter in comparison to the two outer pockets. The two loosely bound $\mathrm{BF}_{4}^{-}$anions could be replaced by halide anions, such as $\mathrm{Cl}^{-}$, leading to the formation of $\mathbf{1 6}$ (Figure 1-22b) through positive cooperativity mimicking the allosteric binding discovered in natural proteins. The chloride anions bind extremely strong with a roughly calculated net binding constant of $10^{20} \mathrm{M}^{-2}$ which allowed complete dissolution of solid $\mathrm{AgCl}$ in acetonitrile.[66]

In order to control the dimerization process and the guest uptake and release, a bulky substituent was introduced to the endohedrally oriented carbonyl position of ligand $\mathbf{1 4}$ yielding ligand 17.[67] Ligand 17 and $\left[\mathrm{Pd}\left(\mathrm{CH}_{3} \mathrm{CN}\right)_{4}\right]\left(\mathrm{BF}_{4}\right)_{2}$ assembled into a monomeric cage $\left[\mathrm{Pd}_{2} \mathbf{1 7}_{4}\right]^{4+}$ instead of an interpenetrated system. Due to the steric hindrance of the attached aryl substituents, the central $\mathrm{BF}_{4^{-}}$, which was essential for templating double cage 15, could not approach the central pocket in this case anymore. However, upon addition of 0.5 equiv. of the smaller $\mathrm{Cl}^{-}$anion to the monomer $\left[\mathrm{Pd}_{2} \mathbf{1 7}_{4}\right]^{4+}$, interpenetration occurred, with $\mathrm{Cl}^{-}$confined inside the resulting central pocket (Figure 1-23). X-ray analysis confirmed the formation of double cage 18. The central cavity of $\mathbf{1 8}$ is significantly shrunk, as result of the binding of $\mathrm{Cl}^{-}$compared to the aforementioned $\mathrm{BF}_{4}^{--}$ templated double cage 15, and both outer pockets become more voluminous. Therefore the enlarged outer pockets of $\mathbf{1 8}$ could better enclose larger oxyanionic guests, such as $\mathrm{ReO}_{4}^{-}$and $\mathrm{ClO}_{4}^{-}$. The allosteric anion binding ability of the two outer cavities is therefore controlled by the size of the anion inside the central pocket. 


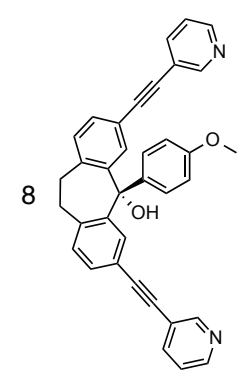

17

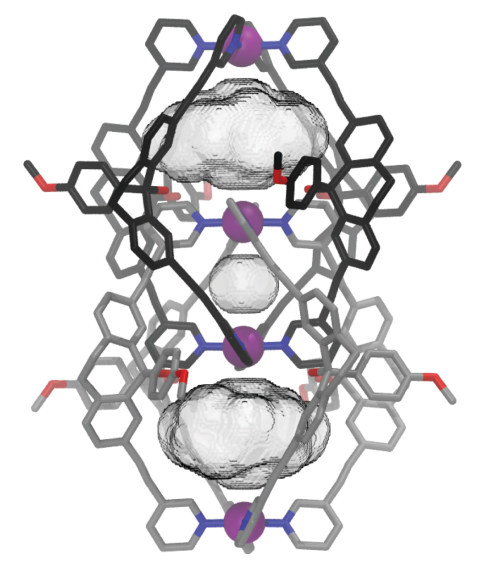

18

Figure 1-23 Bulky ligand 17 and the X-ray structure of the chloride-templated double cage 18. Copyright (C) 2014 The Royal Society of Chemistry.

A similar double cage series including redox active phenothiazine ligands was investigated in detail.[68] The phenothiazine ligand 19a could undergo two- or four-electron oxidation by adjusting the strength of the oxidant. When a mild oxidant, such as $\mathrm{Cu}\left(\mathrm{NO}_{3}\right)_{2} \cdot 3 \mathrm{H}_{2} \mathrm{O}$ was used, mono-oxygenation occurred and sulfoxide ligand $\mathbf{1 9 b}$ was constructed. Under harsher oxidation conditions, reaction with 19a and meta-chloroperbenzoic acid resulted in dioxygenation and formed 19c. Heating each of the phenothiazine ligands 19a-c with $\left[\mathrm{Pd}\left(\mathrm{CH}_{3} \mathrm{CN}\right)_{4}\right]\left(\mathrm{BF}_{4}\right)_{2}$ in 2:1 ratio resulted the formation of double cages 20a-c (Figure 1-24), respectively. Indeed, the mono-oxygenated double cage $20 \mathbf{b}$ was first observed, when the crystals of fully reduced double cage 20a were exposed to air for two months. Alternatively, the oxidized double cages $20 \mathrm{~b}$ and 20c could also be obtained by chemical oxidation in solution. The formation of double cage 20a-c was confirmed by NMR spectroscopy, mass spectrometry, X-ray analysis, UV/Vis, and fluorescence spectroscopy. In the solid state, structural changes between the cages are observed, due to the different oxygenation states of the sulphur atoms. A follow-up study of the phenothiazine and its mono- and dioxygenated cages was carried out by preparing mixed-ligand cages. Mixing the non- 19a and dioxygentaed ligands $19 \mathrm{c}$ with $\mathrm{Pd}^{\mathrm{II}}$ led to the formation of mixed-ligand double cages with a statistical distribution of the ligands. Although the lengths of the ligands are different, they are still tolerated in the mixed cages.[69] 


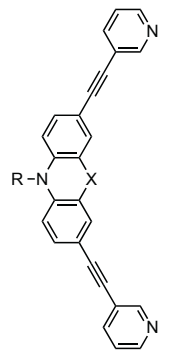

19a $X=S$

19b $\mathrm{X}=\mathrm{S}(=\mathrm{O})$

$19 \mathrm{c} \quad \mathrm{X}=\mathrm{S}(=\mathrm{O})_{2}$

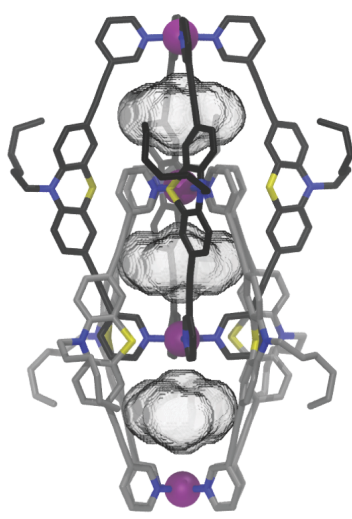

$20 a$

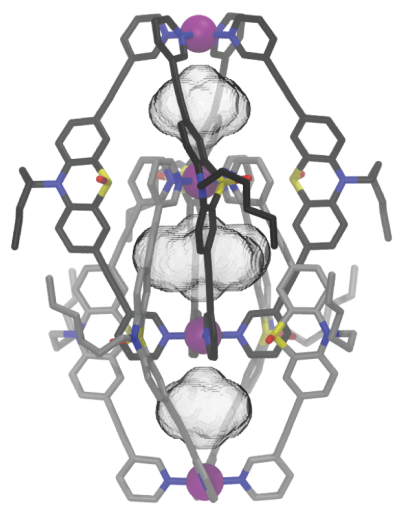

$20 b$

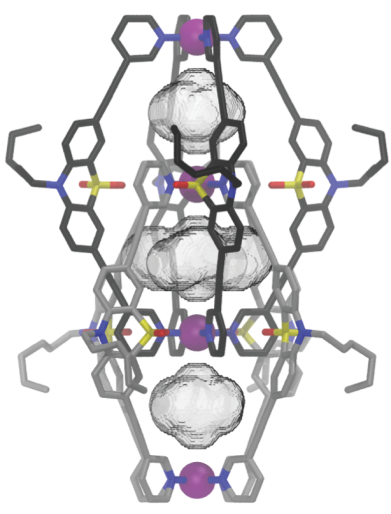

20c

Figure 1-24 Self-assembly and X-ray structures of three related redox active double cages 20a-c based on phenothiazine ligands 19a-c. Copyright (C) 2014 The Royal Society of Chemistry.

As discussed for the aforementioned functions of the halide anions in the self-assembly of double cages, the halide binding was not crucial for dimerization of cages 15,16 and 20 , while the $\mathrm{BF}_{4}^{-}$was considered as the templating anion. But later on, an interesting study based on carbazole backbone containing structures revealed that halide anions could be used as triggers for a structural conversion.

Another bidentate pyridyl ligand $\mathbf{2 1}$ based on a carbazole backbone, which is slightly shorter than the previously discussed phenothiazine and dibenzosuberone ligands, was synthesized. ${ }^{[70]}$ After the addition of $\left[\mathrm{Pd}\left(\mathrm{CH}_{3} \mathrm{CN}\right)_{4}\right]\left(\mathrm{BF}_{4}\right)_{2}$, only stable monomeric cages 22 were constructed. In contrast to the previously mentioned cases, no tendency of dimerization was observed, even in the presence of $\mathrm{BF}_{4}{ }^{-}$anions. The monomeric cage $\mathbf{2 1}$ does not convert to double cage $\mathbf{2 3}$ until the halide anions in stoichiometric amounts were added. NMR spectroscopy and mass spectrometry confirmed the formation of interpenetrated structure 23 , in which three halides are confined in the pockets. The $\mathrm{Pd}_{\text {out }}{ }^{-}$ $\mathrm{Pd}_{\text {in }}$ distance $\left(6.58 \AA\right.$ ) , extracted from a DFT calculation, are distinguishable from the $\mathrm{Pd}_{\mathrm{in}}$ $\mathrm{Pd}_{\text {in }}$ distance $(7.02 \AA)$. Both distances are much shorter than in the previously mentioned double cages. Therefore, only small anionic guests could fit inside the pockets of 23, and induce the dimerization (Figure 1-25). Upon the crystallization of 23, surprisingly, a structure conversion was observed. A triple catenane 24 [trans- $\left.\left(\mathrm{PdBr}_{2}\right)_{2} \mathbf{2 2}_{2}\right]_{3}$ formed and was investigated by X-ray analysis. This triply catenated compound $\mathbf{2 4}$ has no net charge due to the trans-coordination of two bromides at each PdII ion, which allow the palladium atoms to approach each other much closer (Pd-Pd distances between $4.08 \AA$ and $4.78 \AA$ ) 
than in the charged double cages. The six Pd atoms are arranged in an almost linear stack, and the bromide substituents adopt a helical twist.

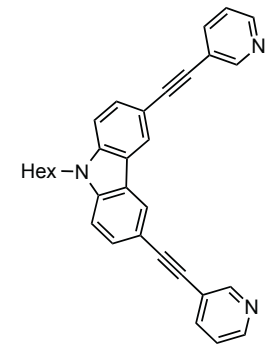

21

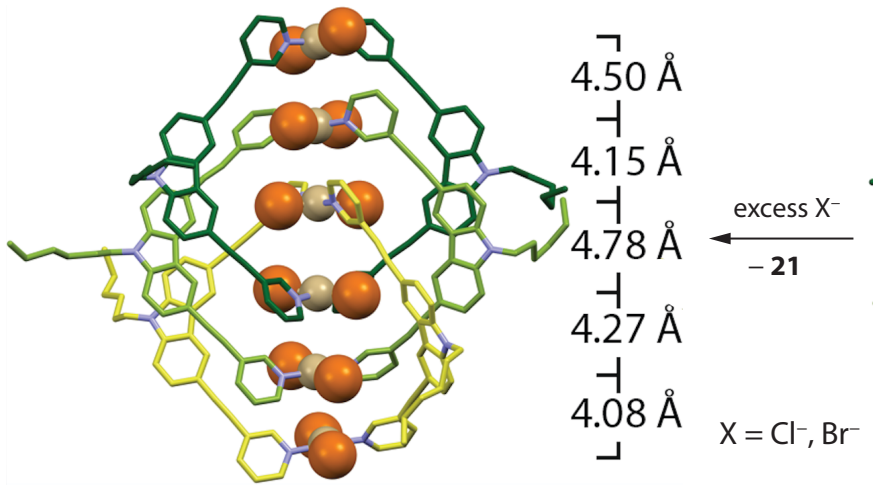

24

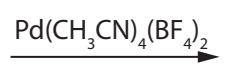

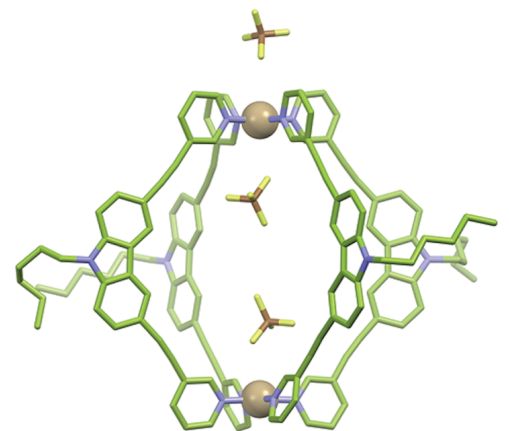

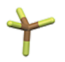

22 1.5 equiv. $X^{-}$

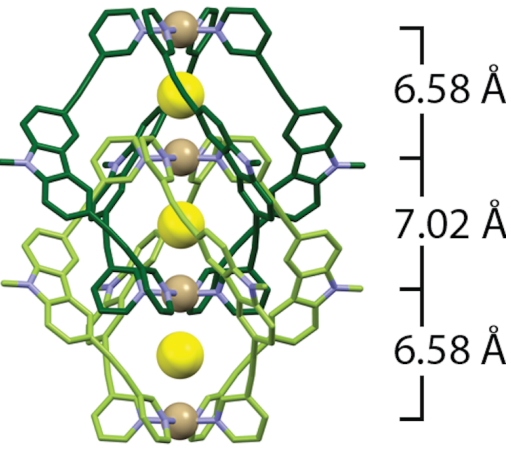

23

Figure 1-25 Stepwise assembly of the monomeric cage 22 (X-ray structure), the templated double cage $23\left[\mathrm{Pd}_{4} \mathbf{2 2}_{8}\right] \supset 3 \mathrm{Cl}$ (DFT calculated structure) and the triple catenane 24 [trans-(PdBr) $\left.{ }_{6} \mathbf{2 2}_{6}\right]_{3}$ (X-ray structure). Copyright (C) 2015 WILEY-VCH Verlag GmbH \& Co. KGaA, Weinheim.

The carbazole-based structures are not the first cage system the Clever group has prepared, which can interconvert between different structures. In Chapter 2, a different self-assembled cage compound and its structural conversion upon crystallization in the presence of large globular anionic guests will be discussed in detail.[71]

\subsubsection{Double trefoil knot}

A double trefoil knot $\mathbf{2 6}$ was unexpectedly realized by the Clever group when they modified a ligand of an already published $\operatorname{Pd}_{2} \mathbf{L}_{4}$ cage ${ }^{[72]}$ in attempts to expand the cages cavity size. Reaction of the modified ligand $\mathbf{2 5}$ with $\mathrm{Pd}{ }^{\mathrm{II}}$ formed a cage-like structure with 
the formula $\left[\mathrm{Pd}_{3} \mathbf{2 5}_{6}\right]$ in a quantitative yield. ${ }^{[73]}$ The non-trivial topology of the double trefoil knot 26 was determined by detailed NMR spectroscopic studies in combination with molecular modelling (Figure 1-26). The cage consists of two hemispheres, which are linked by three PdII ions (Figure 1-26b). Each hemisphere resembles a trefoil knot unit. Besides the metal-ligand interactions, the double trefoil knot $\mathbf{2 6}$ is stabilized through inter-ligand hydrogen bonds and $\pi$-stacking between the aromatic units of the ligands.

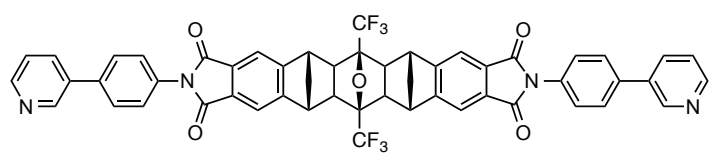

25

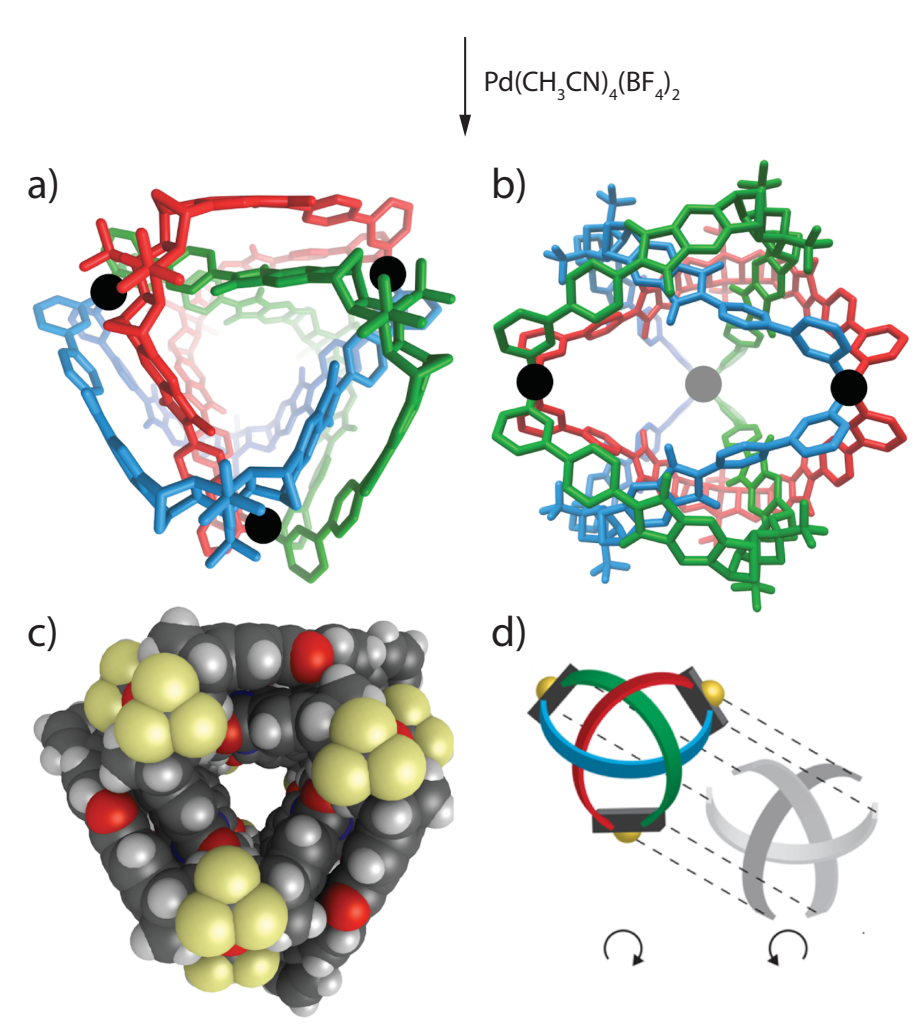

26

Figure 1-26 A double trefoil knot structure 26. Connectivity shown from a) top view and b) side view. c) Space filling of 26. d) Schematic illustration of the underlying topology with the two hemispheres separated. Copyright (C) 2012 WILEY-VCH Verlag GmbH \& Co. KGaA, Weinheim.

\subsubsection{Self-assemblies with lower symmetry}

The rational design of lower symmetry cage structures is a growing research field. This can be achieved either by using more than one kind of metal centre, e.g. in heterometallic assemblies, or by employing a controlled arrangement of different ligands around the metal centres, e.g. in a heteroleptic coordination environment. 
Shionoya and co-workers have reported an impressive example of a heterometallic assembly.[74] They have prepared a mixed metal Tilv and PdII cage $29\left[\mathrm{Pd}_{3} \mathrm{Ti}_{2}\left(\mathbf{2 8}_{2}\right) \mathrm{Cl}_{6}\right]^{4-}$ based on bi-functional pyridyl-catechol ligand 27 (Figure 1-27). Stang and co-workers have designed comparable heterometallic structures, which adopt a trigonal-bipyramidal geometry based on preorganized metalloligands incorporating $\mathrm{Al}^{\mathrm{III}}$ or Ga ${ }^{\mathrm{III}}$ and pyridylacac ligands with $\mathrm{Pt}^{\mathrm{II}}$ acceptors. ${ }^{[75]}$

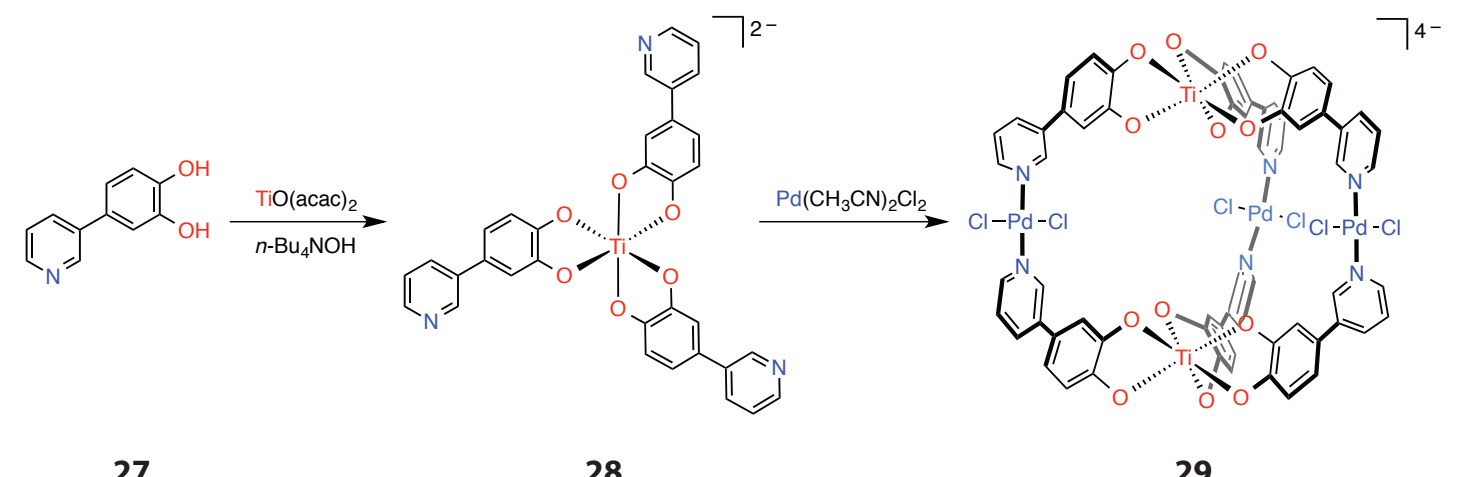

Figure 1-27 Formation of heteronuclear Pd ${ }^{\mathrm{II}}-\mathrm{Ti}^{\mathrm{IV}}$ complex 29.

Meanwhile, plenty of homonuclear and heteroleptic self-assembled structures have been published by Stang, [76,77] Fujita,[78], Schmittel,[79] and others. ${ }^{[80,81]}$ For example, Braxter et al. used oligobipyridine strands and hexaazatriphenylene (HAT) derivatives to coordinate to $\mathrm{Cu}^{\mathrm{I}}$ or $\mathrm{Ag}^{\mathrm{I}}$ ions in order to achieve cylindrical structures with individual nanometric sizes.[82] Shionoya and co-workers synthesized a porphyrin hexameric cage using Znporphyrin ligand, which includes three different kinds of heteroleptic bpy- $\mathrm{Zn}^{\mathrm{II}}{ }^{\mathrm{units}}{ }^{\left[{ }^{83}\right]}$ Recently, the Clever group reported an interesting ligand design which allowed the synthesis of a unique square-cuboid structure, in which only a single metal salt was used, and all the coordination environment of the metal ions are identical.[84] The design strategy, mathematical analysis, synthesis and characterization of the face-centred squarecuboid assembly will be described in Chapter 3 .

\subsection{Applications of supramolecules}

Metal-based supramolecular coordination architectures have attracted a great deal of interest from supramolecular chemists over the past decades because of their ability to mimic biological processes and their potential applications.[85,86] The inner cavities create a unique environment and could provide different interactions with small guest molecules than these would experience in free solution. 


\subsubsection{Molecular recognition and encapsulation}

Supramolecular self-assemblies, such as cages and capsules, can be obtained by complexation of a wide range of metal components and organic ligand molecules. These supramolecules are able to encapsulate various types of guest molecules depending on the chemical nature and the size of their inner cavities.

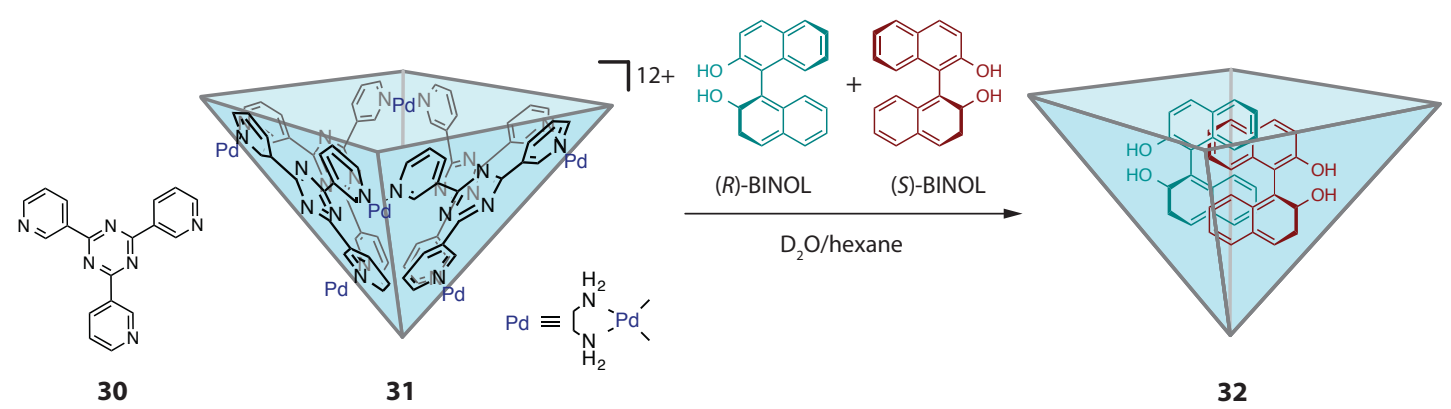

Figure 1-28 Molecular recognition of enantiomers $(R)$-BINOL and $(S)$-BINOL in host 31.

Fujita and co-workers used a bowl-shaped coordination host compound 31, which was self-assembled from $\left[\mathrm{Pd}(\mathrm{en})\left(\mathrm{NO}_{3}\right)_{2}\right]$ and tris(3-pyridyl)triazine ligand $\mathbf{3 0}$ in a 6:4 ratio,[87] to investigate the chiral recognition of 1,1'-bi-2-naphthol (BINOL).[88] The combination of an aqueous solution of the host 31, with a solution of $(S)$-BINOL in hexane at a $50 \%$ enantiomeric excess $(e e)$ led to the formation of a host-guest complex 32 ([(R)-BINOL· $(S)$ BINOL]@31), which encapsulated 1 equiv. of racemic mixture (1:1) selectively within its cavity (Figure 1-28). The bimolecular heterorecognition of enantiomers afforded a significant chirality enrichment of BINOL in the organic phase from $50 \%$ to $87 \% e e$ without addition of any other source of chirality.

Another interesting example is the use of a dinuclear macrocycle to encapsulate ditopic aromatic guest molecules, [2.2]paracyclophane and ferrocene.[89] Reaction of a cyclophane-type macrocyclic ligand 33 and 2 equiv. $\mathrm{AgSbF}_{6}$ yielded initially the dinuclear AgI complex $34\left[\mathrm{Ag}_{2} 33 \mathrm{X}_{2}\right]\left(\mathrm{SbF}_{6}\right)_{2}$. The AgI complex binds ditopic guests strongly within its cavity and forms highly stable inclusion complexes 35 and 36 (Figure 1-29) through multipoint AgI- $\pi$ interactions. Furthermore, the electrochemical behaviour of ferrocene was changed remarkably upon encapsulation. Since the ferrocene was recognized and fixed between the two AgI centres of the macrocycle, the electrostatic repulsion between the $\mathrm{AgI}$ ions and the oxidized ferrocenium $\left(\mathrm{FeCp}_{2}{ }^{+}\right)$made the inclusion complex with $\mathrm{FeCp}_{2}{ }^{+}$less stable compared with the neutral ferrocene $\left.(\mathrm{FeCp})_{2}\right)$. 


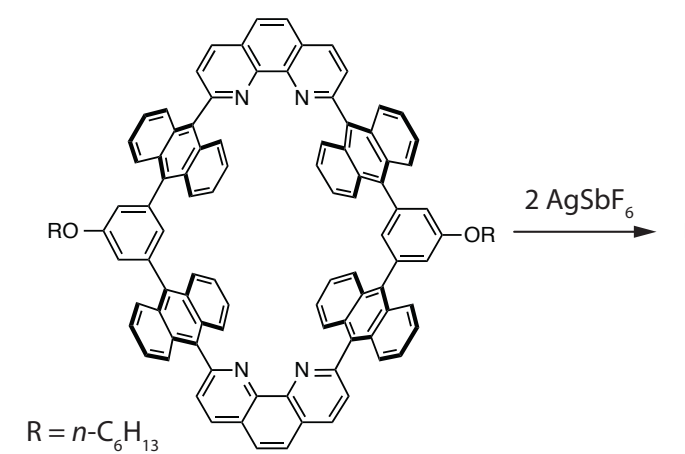

33

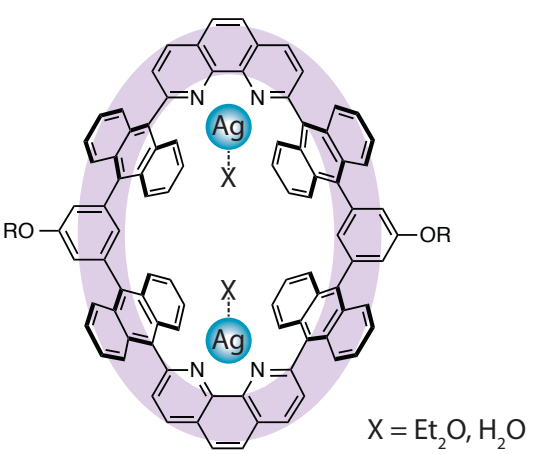

34

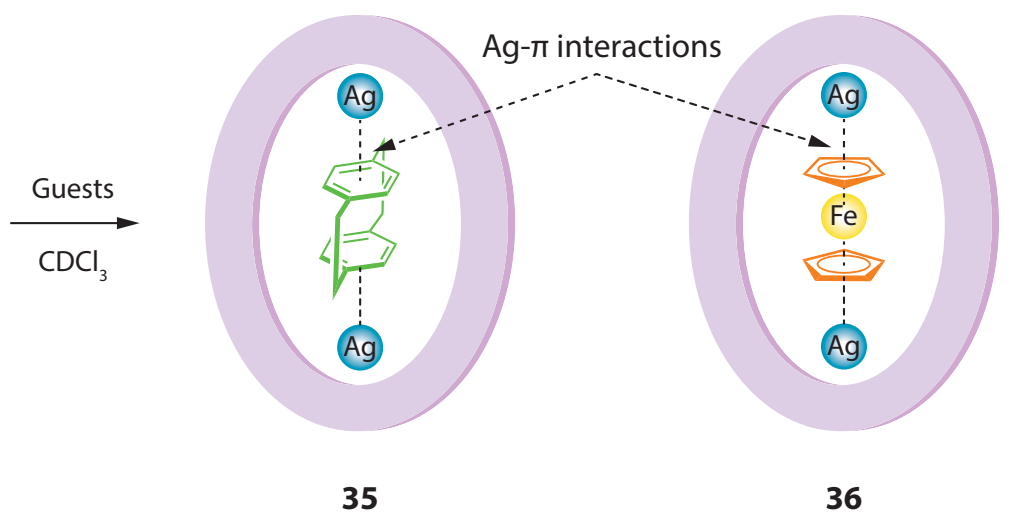

Figure 1-29 Synthetic access to dinuclear AgI-macrocycle $34\left[\mathrm{Ag}_{2} 33 \mathrm{X}_{2}\right]\left(\mathrm{SbF}_{6}\right)_{2}$ and schematic illustration of the inclusion complexes.

Additionally, some supramolecular cages were also found to encapsulate polycyclic aromatic hydrocarbons (PAHs), ${ }^{[00-92]}$ which are considered as one of the most widespread organic environmental pollutants.[93]

\subsubsection{Reactivity modulation}

Supramolecular self-assemblies are able to modify the reactivity of the guest molecules they incarcerate. For instance, they could stabilize unstable molecules and reactive intermediates within their cavities, such as iminium,[52] phosphonium,[94] nucleaobase pairs or even small nucleotide duplexes.[95]

Nitschke and co-workers demonstrated that a self-assembled water-soluble tetrahedral cage 37 could encapsulate white phosphorus $\mathrm{P}_{4}$, which is highly pyrophoric, and consequently prevent it from oxidation in both solution and in the solid state (Figure 1-30).[96] As soon as the host-guest complex 38 was formed, the oxygen molecules could not affect $\mathrm{P}_{4}$ anymore, because the cavity of $\mathbf{3 7}$ is not large enough for phosphorus oxidation to occur. The encapsulated $\mathrm{P}_{4}$ could be released without disrupting the cage $\mathbf{3 7}$ 
by adding a competitive guest such as benzene. Once $\mathrm{P}_{4}$ molecules were replaced by benzene 40, they were immediately oxidized and hydrolysed to phosphoric acid.

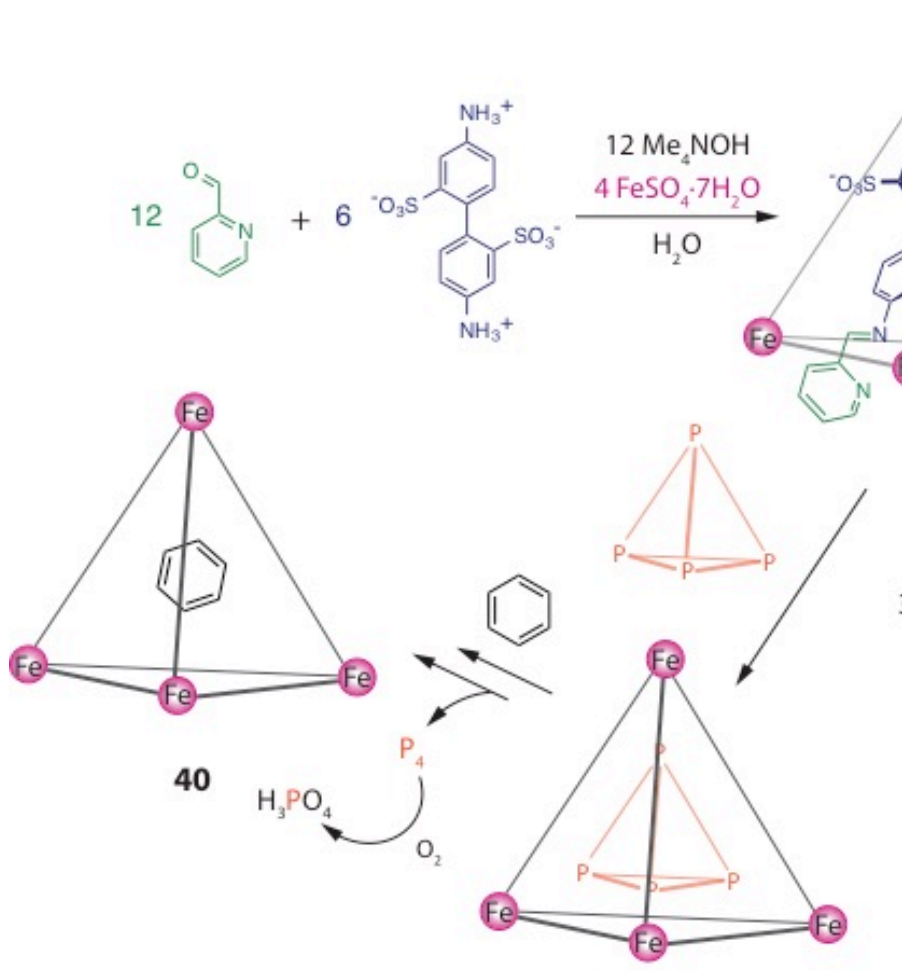

38

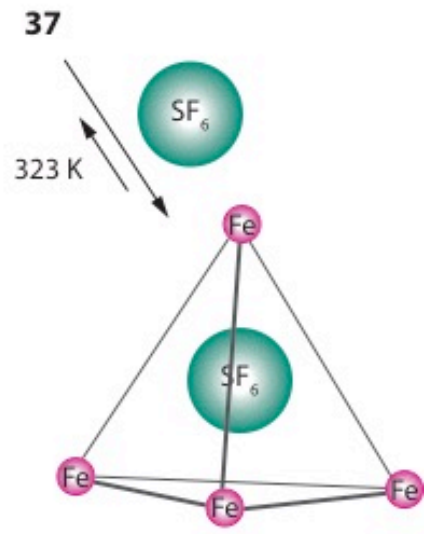

39

Figure 1-30 Tetrahedral molecular cage 37 and its host-guest chemistry. Copyright (C) 2009 American Association for the Advancement of Science.

Besides, the same tetrahedral cage $\mathbf{3 7}$ was found to be able to encapsulate the most potent green house gas $\mathrm{SF}_{6}$ very strongly with a binding constant up to $10^{4} \mathrm{M}^{-1}$ (Figure 1-30).[97] Competitive guests were used to examine the selective binding with the cage compound, but no binding was observed when the cage 37 was exposed to $\mathrm{Xe}, \mathrm{Ar}, \mathrm{N}_{2}, \mathrm{O}_{2}, \mathrm{C}_{2} \mathrm{H}_{4}, \mathrm{CO}_{2}$ and $\mathrm{N}_{2} \mathrm{O}$. Heating the system to $323 \mathrm{~K}$ broke the strong binding of the $\mathrm{SF}_{6}$ and the cage 37 could be rebuild. This reversible and selective binding control could be employed in gas separation or $\mathrm{SF}_{6}$ recycling.

\subsubsection{Molecular sensing}

The use of supramolecular systems in sensing has received increasing attention by several research groups.[98-100] The host-guest interaction could be tracked and measured by a chemical or physical change of the receptor that in some cases can be easily observed.[75,101] 
Severin and co-workers presented the synthesis of a cylindrical imine-based cage $\mathbf{4 1}$

(Figure 1-31) constructed out of two trimeric metallomacrocycles. The cage showed impressive binding ability with alkali metal cations and acted as a heterotopic host.[102] The cage binds small cations, such as $\mathrm{Li}^{+}, \mathrm{Na}^{+}$and $\mathrm{K}^{+}$(Figure 1-31b) at the outside of the cage while larger ions like $\mathrm{Rb}^{+}$and $\mathrm{Cs}^{+}$inside the cage (Figure 1-31c). Only the complexation of $\mathrm{Cs}^{+}$is correlated with a color change, therefore the cage could be applied as a selective sensor for $\mathrm{Cs}^{+}$by the 'naked-eye'.

a)

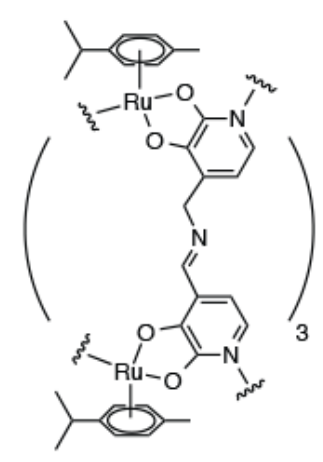

41 b)

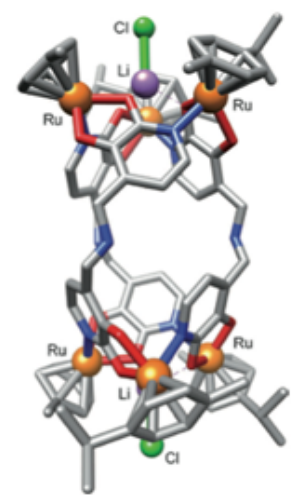

42 c)

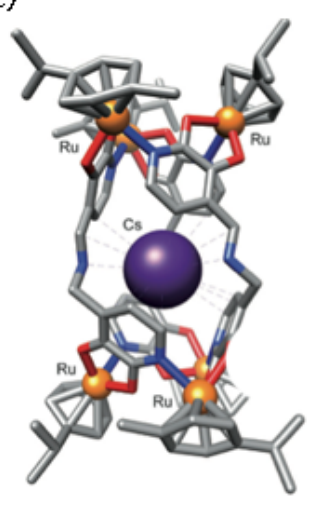

43

Figure 1-31 a) Structure of cylindrical imine-based cage 41. Molecular structures of b) 42: bis-LiCl and c) 43: $\mathrm{Cs}^{+}$adducts of cage $\mathbf{4 1}$ in the solid state. Copyright (C) 2013 WILEY-VCH Verlag GmbH \& Co. KGaA, Weinheim.

For instance, Yamashita et al. synthesized a RuII-based capsule by treatment of 2,4,6-tri(4pyridyl)-1,3,5-triazine and $\left[\mathrm{Ru}\left([12]-\mathrm{aneS}_{4}\right)\left(\mathrm{H}_{2} \mathrm{O}\right)(\mathrm{DMSO})\right]\left(\mathrm{NO}_{3}\right)_{2} \cdot[103]$ The cage showed interesting sensing properties after 1-adamantanol was encapsulated, the orange solution turns red, which allow the usage as a colorimetric sensor. Another interesting example of a fluorescence senor was developed by Stang and co-workers. ${ }^{[75]}$ Trigonal-prismatic cages were constructed by combining dinuclear half-sandwich octahedral $\mathrm{Ru}^{\mathrm{II}}$-arene linkers and 1,3,5-tris-(4-pyridylethenyl)benzene. The fluorescence emission of these electron-rich cages was quenched upon addition of electron-deficient nitro-aromatics.

\subsubsection{Catalysis and reactions}

Design and understanding of catalytic processes is one of the major endeavours of supramolecular chemistry. The supramolecular assemblies enable the studies of reactivity and properties of molecules within well-defined confined spaces. It has been proven that covalently build cages or capsules can be used as catalysts for a wide range of chemical reactions[104] with impressive efficiencies and selectivities.[105] 


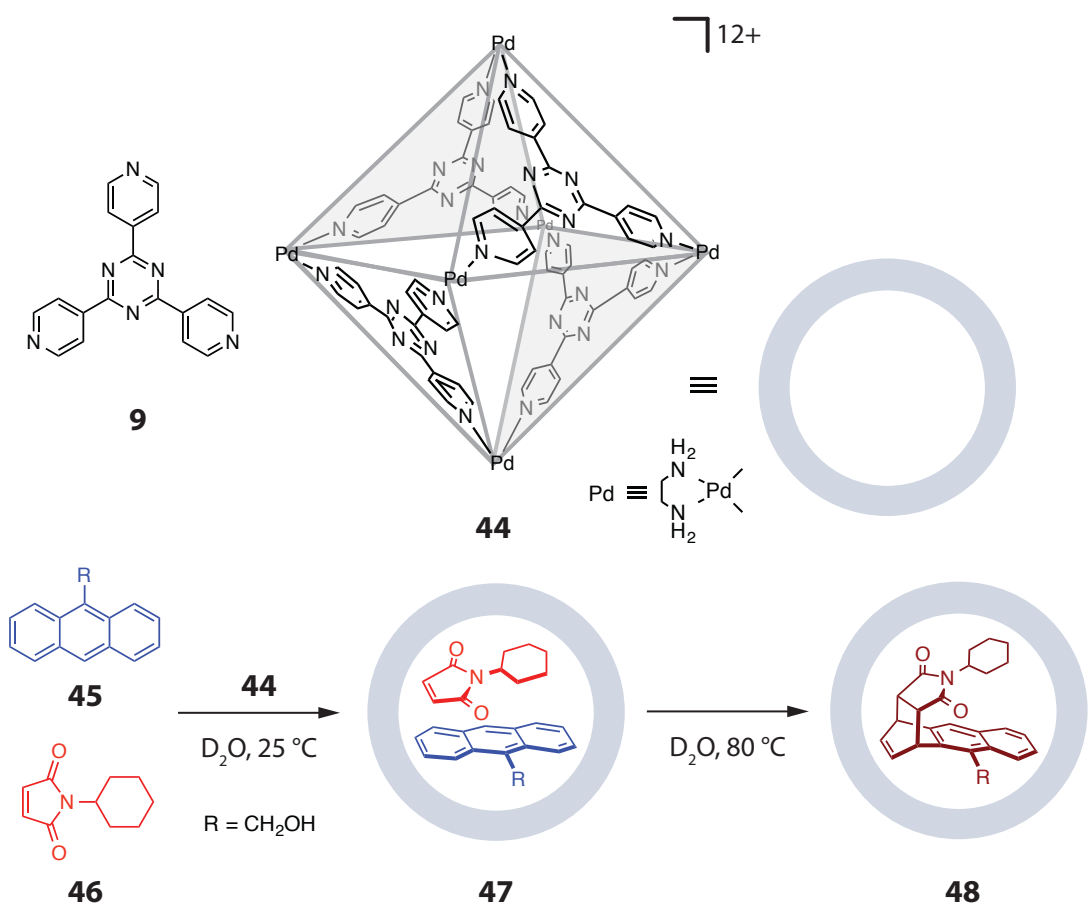

Figure 1-32 Diels-Alder reaction of anthracene and $N$-cyclohexylmaleimide within 44.

Fujita and co-workers employed the octahedral cage $\mathbf{4 4}^{[106]}$ as a molecular flask for the Diels-Alder reaction between anthracenes and maleimides.[53] In the solution of cage 44, when diene 45 and dienophil $\mathbf{4 6}$ were present stoichiometrically at room temperature, the 1:2 host-guest complex $\mathbf{4 7}$ was formed selectively in the solution (Figure 1-32). After heating the solution for $5 \mathrm{~h}$ at $80{ }^{\circ} \mathrm{C}$, unusual regio- and stereoselective Diels-Alder syn1,4-adduct 48 was observed in a very good yield (98\%), although the diene anthracene reacts typically and selectively with dienophiles to the 9,10-adduct.[107] However, the product inhibition prevents this reaction showing turnover although the stereo- and regioselectivities of the product are well controlled. If using the bowl-shaped host $\mathbf{3 1}$ instead, efficient catalytic turnover could be observed with autoinclusion of substrates $\mathbf{4 5}$ and 46 and autoexclusion of the product. In this case, however, the 9,10-adduct was formed.

Recently, Zhang and Tiefenbacher used a Brønsted acidic capsule assembled using hydrogen bonds. This capsule was first introduced by Atwood in 1997 and consists of six calix[4]resorcinarenes.[108] here it was used to catalyse tail-to-head cyclization of polyene substrates.[109] Another recent highlight is the immobilization of a chiral monophosphoramidite- $\mathrm{Rh}^{\mathrm{I}}$ catalyst in a nonchiral heteroleptic and heteronuclear $\mathrm{Pd} / \mathrm{Zn}$ cage.[110] In comparison to the non-encapsulated Rh catalyst, this resulting supramolecular catalyst hydroformylates styrene and derivatives into the corresponding aldehydes with impressively enhanced chiral induction. 


\subsubsection{Biological and medical applications}

A great deal of attention has been put into the field of biological and medical applications of self-assembled supramolecular systems. Until now, many research groups have applied coordination-driven supramolecular assemblies by incorporating biological or medicalrelated functionalities into them, such as supramolecular DNA recognition,[111] recognition and folding of peptides,[112] and encapsulation of proteins.[113,114]

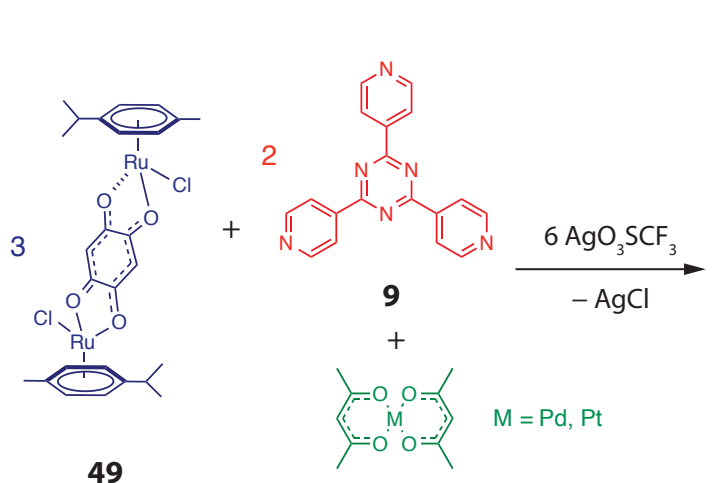

50

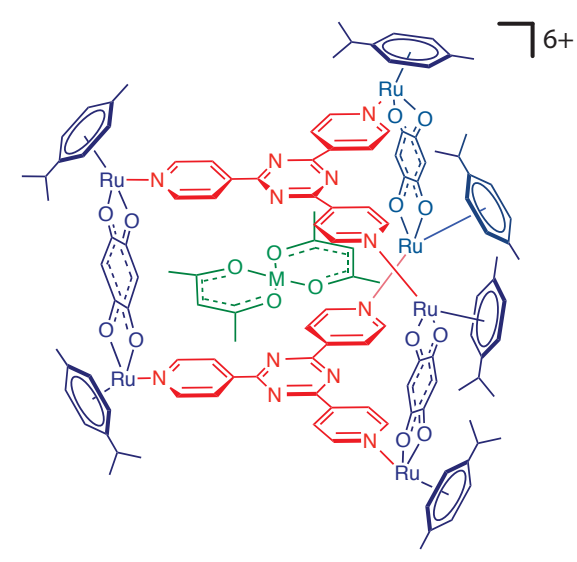

51

Figure 1-33 Synthesis of the heteroleptic trigonal prismatic $\mathrm{Ru}^{\mathrm{II}}$ cage $\mathbf{5 1}$ showing encapsulated $\mathrm{M}(\mathrm{acac})_{2}$ guest.

Therrien and co-workers reported a trigonal prismatic hexanuclear cage 51, in which six ( $\eta^{6}$-arene)-coordinated ruthenium ions could bring together three 2,5-dihydroxyl-1,4benzoquinonato linkers 49 and two trigonal tris(4-pyridyl)triazine panels 9 (Figure 1-33).[115] Though this cage has been found to encapsulate a range of aromatic guests,[116,117] it became more attractive when guests with biological applications, such as $50 \mathrm{M}(\mathrm{acac})_{2}(\mathrm{M}=\mathrm{Pd} / \mathrm{Pt}$; acac = acetylacetonato $)$ could also be successfully encapsulated in the prismatic cage.[115] The cytotoxic activity of the "complex-in-a-complex" systems $\mathbf{5 1}$ was estimated on human ovarian A2780 cancer cells. The encapsulation gave the possibility for the non-water soluble $\mathrm{M}(\mathrm{acac})_{2}$ molecules to be taken up by cancer cells. The species $\mathbf{5 1}$ is about twice as active as the empty cage complex, and the inclusion complexes were more cytotoxic.

In a separate study by Crowley and co-workers, a dipalladium molecular cage has been used to confine two equivalents of anti-cancer drug cis-platin..[118] The cage itself is stimuliresponsive and can be reversibly dis- or reassembled upon addition or cleavage of suitable competitive ligands. Recently, Cullen et al. used the pH-dependent binding of an octanuclear cubic $\mathrm{Co}^{\mathrm{II}}$ based cage for a reversible uptake/release of Parkinson's drug 1amino-adamantane by a pH swing.[119] 


\subsubsection{Molecular switches}

Over the past decade, chemists devoted tremendous effort on the synthesis and modification of molecular switches. From a targeted and controllable uptake or release of specific active substances in biological systems,[120] over molecular transistors, information storage, logic gates and to non-destructive readout, molecular switches exhibit a variety of potential applications.[121,122] The fundamental version of these molecular switches can be the interconversion from one state to another, by external stimuli, such as light irradiation,[123] change in the electric potential[124], redox [125] or pH.[126] These two states could be differentiated through their absorption spectra and geometries. Along with the change of absorption spectra and geometries, commonly changes of the physical properties such as solubility, viscosity, refractive index and dielectric constant are observed.[127] Especially, light is considered as the most suitable stimulus because of the convenience of controlling the irradiation area and time, precise manipulation of the wavelength and intensity, and no contamination.[120]

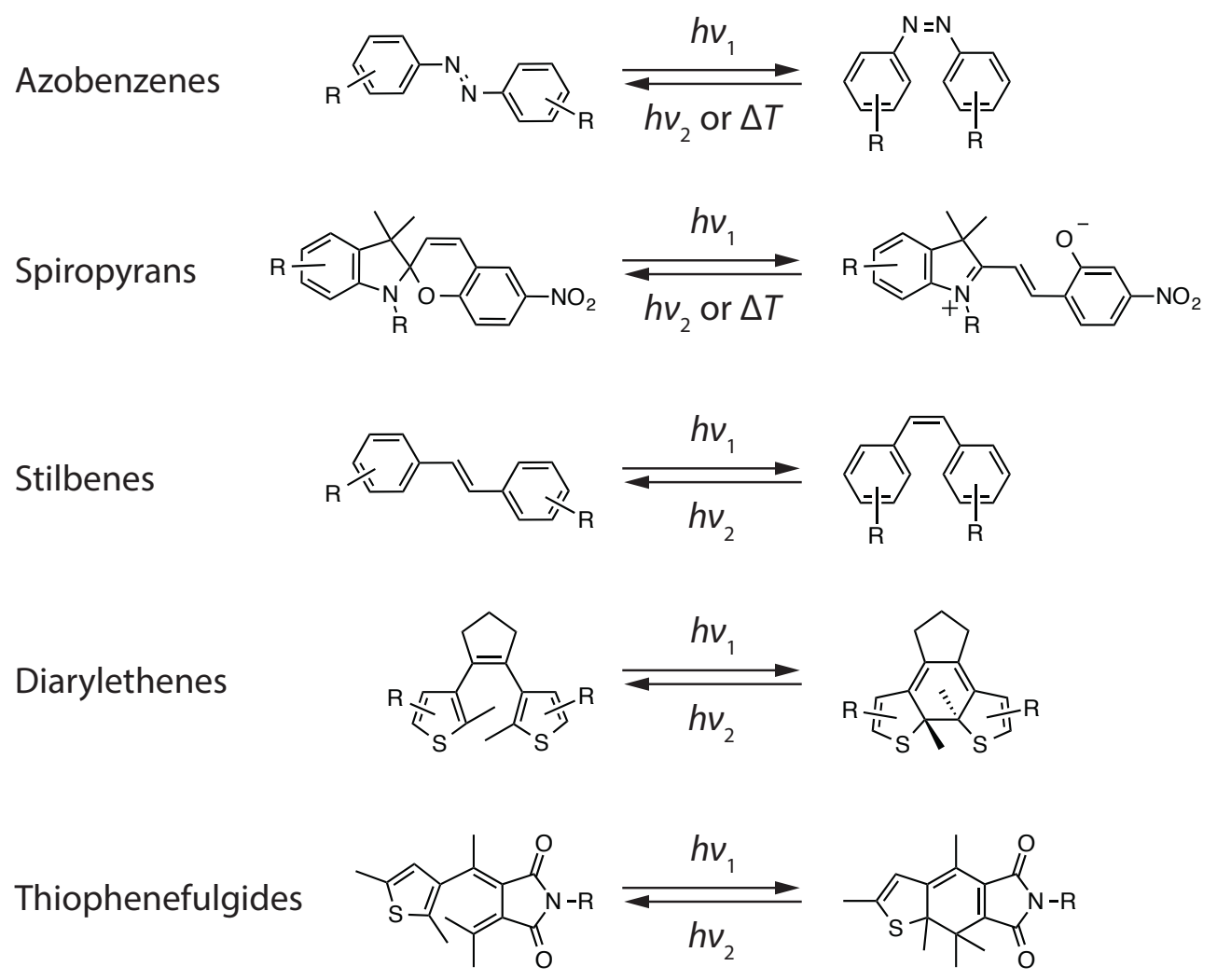

Figure 1-34 Selected structures of photoswitches (photochromic molecules).

A large set of photochromic compounds have been designed and synthesized, which can undergo reversible structural changes upon irradiation with light (Figure 1-34). Some photochromic molecules, such as azobenzenes and spiropyrans, could be switched 
reversibly between their colourless and coloured isomers by irradiation. The photogenerated isomers are thermally unstable and can be converted back to the colourless isomers thermally. In contrast, stilbenes, fulgides, diarylethenes undergo thermally irreversible processes. The switch of the photochromic molecules occurs between the cis- and trans-isomers (azobenzenes and stilbenes), which causes large geometry changes, or between the ring-open and closed forms (spiropyrans, fulgides, and diarylethenes), which results in small geometry change.

\subsubsection{Switchable hosts}

Such photoactive functional molecular photoswitches were combined with host compounds, such as calixarens, cyclodextrins and crown ethers, to achieve a number of attractive photoresponsive systems, ${ }^{[128]}$ such as molecular tweezers ${ }^{[129,130]}$ and scissors,[131] molecular rotors and machines.[132]

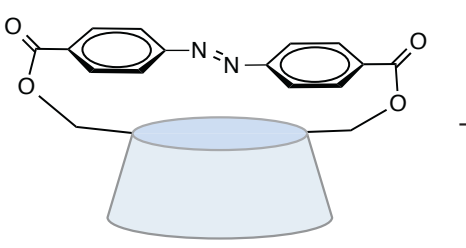

trans-52

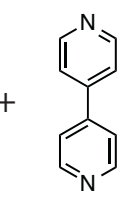

53

3

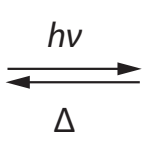

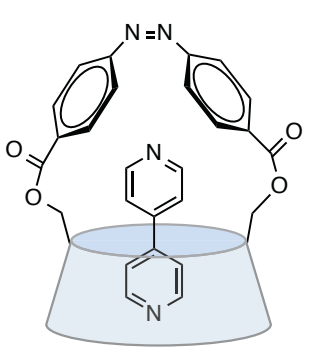

53@cis-52

Figure 1-35 Azobenzene-capped $\beta$-cyclodextrin 52 as photoswitchable host.

Ueno and co-workers described the combination of an azobenzene-capped $\beta$-cyclodextrin $(\beta$-CD) 52 whose guest binding ability is modified by photoisomerization of the $\mathrm{N}=\mathrm{N}$ double bond of the azobenzene.[133] Upon irradiation, trans-52 with small cavity size was converted into its cis-form, which exhibits a much larger cavity. Depending on the guest molecules, the host system was found to have different binding affinities. Larger guests, such as 4,4'-bipyridine $\mathbf{5 3}$ could be encapsulated in a 1:1 ratio only in cis-52, whereas the trans-52 could not bind 53 at all (Figure 1-35). 


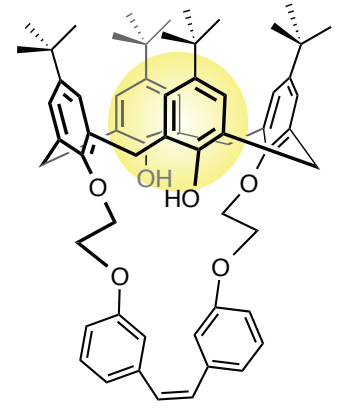

55@cis-54

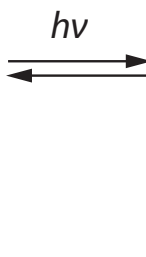

trans-54

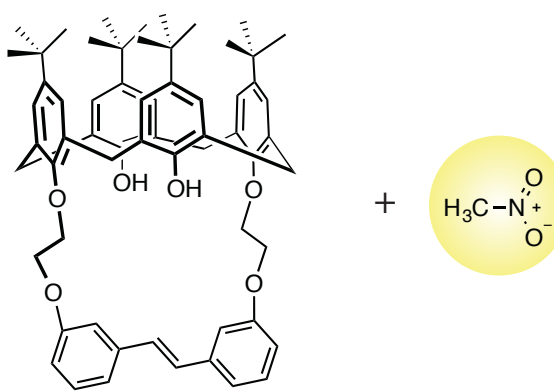

55

Figure 1-36 Photoresponsive stilbene-bridged $p$-tert-butylcalix[4]arene 54 as host.

Rojanathanes et al. reported a photoresponsive host system, using a stilbene-bridged calix[4]arene. The cis-isomer of $\mathbf{5 4}$, allows its calixarene part to encapsulate small electron-deficient guest molecules, such as nitromethane $\mathbf{5 5}$ and acetonitrile. After irradiation, the host cis-54 converted to its trans-form, which twisted the cavity resulting the release of the guest compound (Figure 1-36).[134]

Fujita and co-workers prepared a spherical cage with 24 azobenzenes attached at the interior of the cage cavity.[135] Upon irradiation the hydrophobicity of the interior of the spherical cage could be controlled through the reversible photoisomerization of azobenzenes.

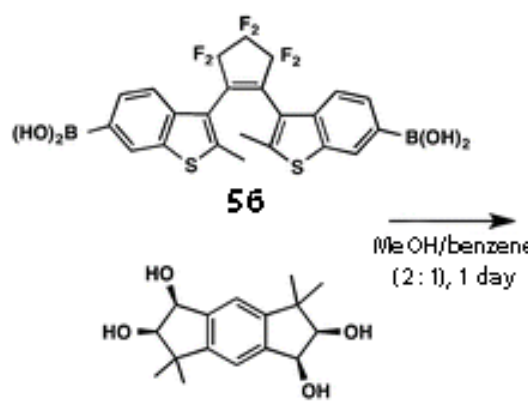

$\operatorname{rac}-57$

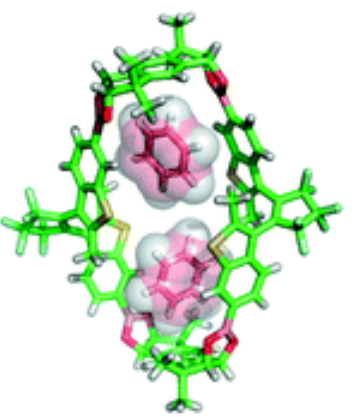

58

Figure 1-37 Self-assembly of macrocyclic boronic ester 58 containing diarylethene units 56 and rac-57. Copyright (C) 2012 The Royal Society of Chemistry.

An interesting macrocyclic boronic ester was reported by Uekusa and co-workers. By mixing open-form diarylethene containing 56 and racemic 1,2-diol units rac-57, only in the presence of benzene as part of the solvent, a macrocycle $\mathbf{5 8}$ could be isolated (Figure 1-37).[136] The guest-induced self-assembly of 58 showed high quantum yield of photoisomerization due to the favorable constraint of the conformation of the diarylethene units. 
a)

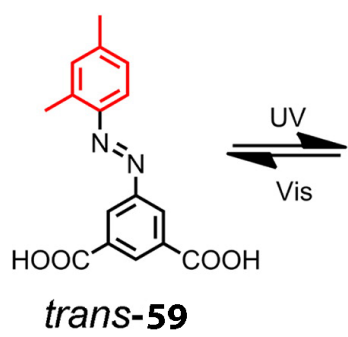

b)

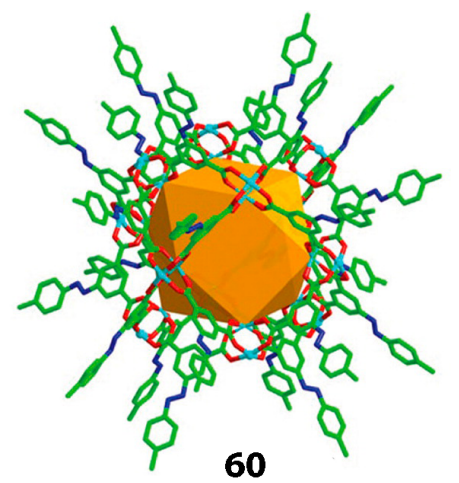

c)

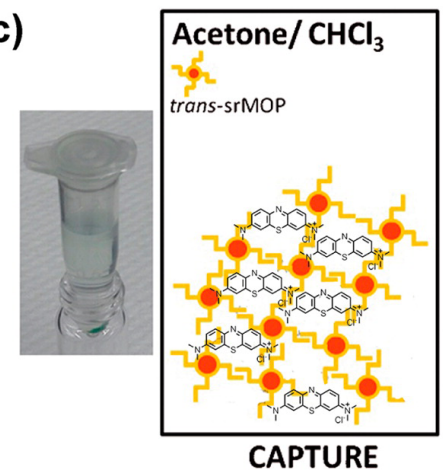

cis-59

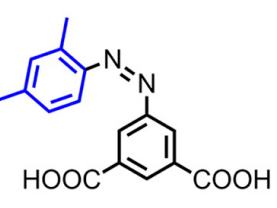

59

60

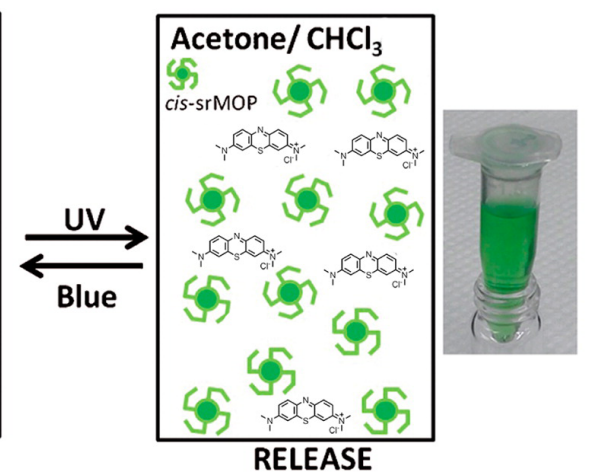

Figure 1-38 a) Light induced trans-cis isomerization of azobenzenes contained organic linker 59 b) X-ray structure of azobenzene modified cuboctahedral polyhedra 60. c) Schematic demonstration of the reversible capture and release of methylene blue guest from photoresponsive 59 upon irradiation in different wavelength. Copyright (C) 2014 WILEY-VCH Verlag GmbH \& Co. KGaA, Weinheim.

Park et al. reported a photoresponsive metal-organic polyhedron (MOP) 60 constructed of organic linkers containing azobenzenes 59 and $\mathrm{Cu}(\mathrm{OAc})_{2}$ (Figure 1-38a, b). The MOP 60 can be switched reversibly between trans- and cis-isomer in response to light irradiation, and resulted in size and solubility change. This result encouraged them to control the capture and release of guest molecules. Methylene blue was selected as a suitable guest molecule, which is too large to be confined in the cavity of small polyhedron cis-60. The capture process of methylene blue into the pockets between trans-60 units could be followed by UV-Vis spectroscopy. Upon irradiation at $365 \mathrm{~nm}$, the insoluble trans-59 interconverted to the soluble cis-60, the strong interactions between the trans-60 did not exist anymore, methylene blue guests were released along the formation of cis-60 (Figure 1-38c). Besides, this process can reverse back under blue light irradiation. The capture and release of the methylene blue guests are highly reversible only by alternating the wavelength of the irradiation.[137] 


\subsubsection{Switchable guests}

Rebek and co-workers explored the photochemical control of reversible encapsulation based on a hydrogen-bonded cylindrical dimeric capsule by using 4,4'dimethylazobenzene as light-switchable guest.[138] The trans-isomer could fit perfectly into the capsule cavity, whereas the cis-isomer could not. Indeed, the bent cis-isomer of the guest clashes with the capsule, breaks its hydrogen bonds and leaves the capsule consequently. After this process, other potential guests, such as $n$-tridecane, can be encapsulated in the capsule cavity.

a)

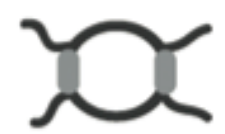

$61 \mathrm{a}$

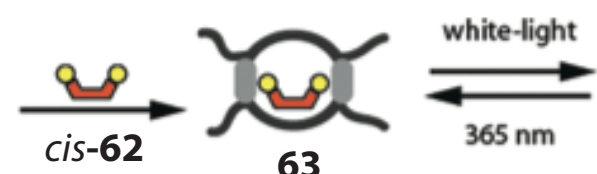

63

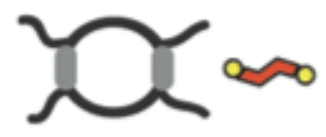

$61 a+$ trans-62

b)

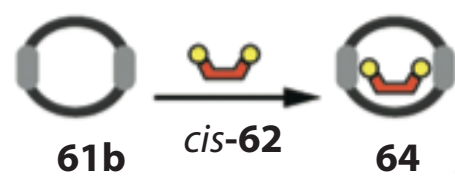

\section{c)}

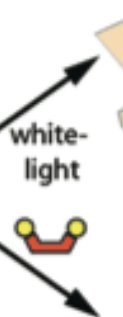

e)
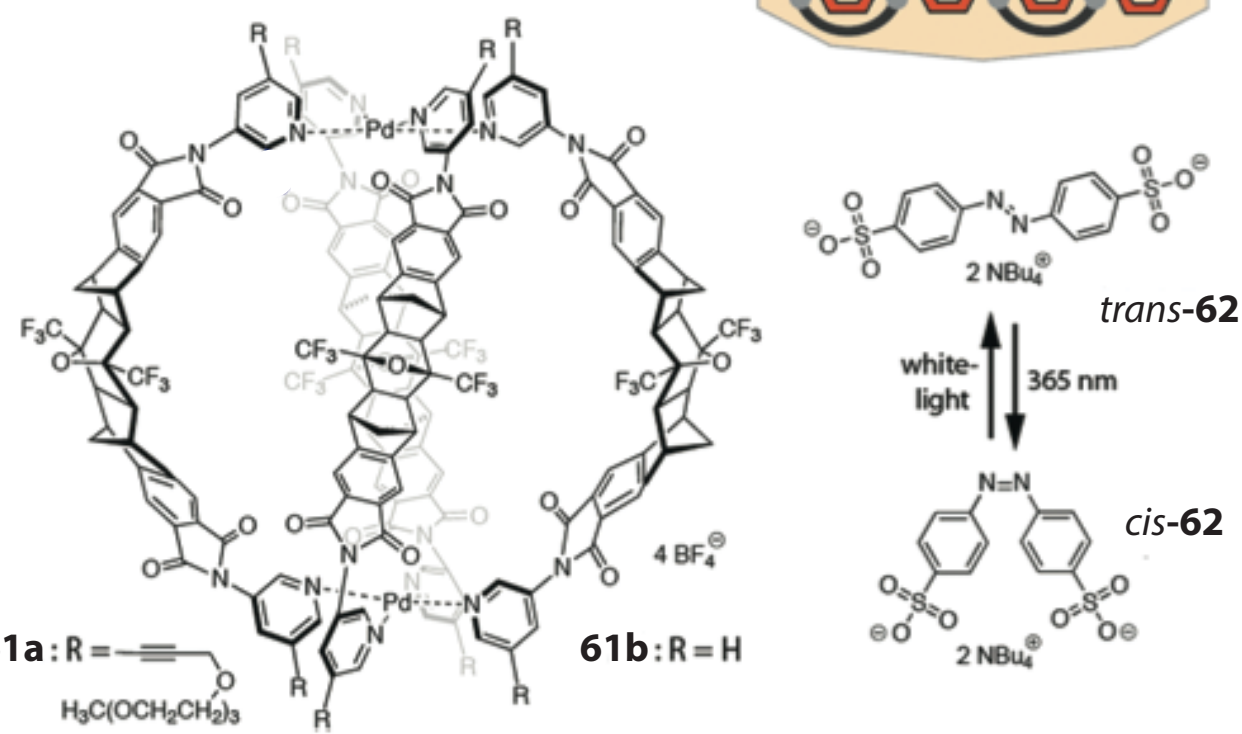

Figure 1-39 a) Encapsulation of cis-62 by the PEG cage 61a and the reversible host-guest photoswitching. b) Quantitative encapsulation of cis-62 by cage $\mathbf{6 1 b}$ c) Photoisomerization induced crystallization of $\left[(\text { trans }-62)_{2}(\mathbf{6 1 b})\right]_{\mathrm{n}}$. d) Crystallization of $\left[(\text { cis-62 })_{2}(61 \mathrm{~b})\right]_{\mathrm{n}}$ by adding a second equivalent of cis-62. e) Chemical structure of cage $\mathbf{6 1}$ and guests 62. Copyright (C) 2010, American Chemical Society.

The photoisomerization of guest molecules has also been studied by Clever et al. who designed molecular cages 61 (61a with PEG chains for solubility enhancement, 61b 
without any substituents) formed by four rigid concave ligands based on an annulated norbornene backbone and two square-planar coordinated $\mathrm{Pd}^{\mathrm{II}}$ or PtII ions (Figure 1-39e). These cages could encapsulate suitable aromatic bis-sulfonate guests.[139] In this system, a 4,4'-azobenzene bis-sulfonate $\mathbf{6 2}$ was used as photoresponsive guest molecule in order to achieve a reversible light-triggered uptake and release process.[140] The cis-62 could be perfectly encapsulated into the cavity of $\mathbf{6 1 a}$, and form host-guest complex $\mathbf{6 3}$. When the solution of $\mathbf{6 3}$ was irradiated by white light, cis-62 was isomerized into trans-62, which is too long to be enclosed in $\mathbf{6 1 a}$, thus results in the guest release. After irradiation at 365 $\mathrm{nm}$, the host-guest complex 63 can be regenerated (Figure 1-39a). The cage 61b without PEG chains attached could encapsulate cis-62 and form the host-guest complex 64 quantitatively as well. However, when host-guest complex 64 was irradiated with white light, yellow crystals of $\left[(\text { trans-62 })_{2}(\mathbf{6 1 b})\right]_{\mathrm{n}}$ formed immediately. Furthermore, upon addition of a second equivalent of cis-62 guest, a crystallization was observed with the formation of $\left[(\text { cis-62 })_{2}(\mathbf{6 1 b})\right]_{\mathrm{n}}$ (Figure 1-39b). This light-induced phase changing process provides the possibility for developing new strategies for nano-construction.

\subsection{References}

[1] J.-M. Lehn, Angew. Chem. Int. Ed. 1988, 27, 89.

[2] J.-M. Lehn, Supramolecular Chemistry: Concepts and Perspectives, 1st ed., Wiley, Weinheim, Germany, 1995.

[3] A. Villiers, C. R. Hebd, Seances. Acad. Sci. 1891, 112, 435.

[4] A. Villiers, C. R. Hebd, Seances. Acad. Sci. 1891, 112, 536.

[5] A. Werner, Z. Anorg. Allg. Chem. 1893, 3, 267.

[6] E. Fischer, Eur. J. Inorg. Chem. 1894, 27, 2985.

[7] D. E. Koshland, Proc. Natl. Acad. Sci. U.S.A. 1958, 44, 98.

[8] P. Ehrlich, Studies on Immunity, Wiley, New York, 1906

[9] K. L. WOLF, R. WOLFF, Angew, Chem. 1949, 61, 191.

[10] C. J. Pedersen, J. Am. Chem. Soc. 1967, 89, 7017.

[11] B. Dietrich, J. M. Lehn, J. P. Sauvage, Tetrahedron Lett. 1969, 10, 2885.

[12] D. J. Cram, T. Kaneda, R. C. Helgeson, G. M. Lein, J. Am. Chem. Soc. 1979, 101, 6752

[13] F. Crick, J. Watson, Nature 1953, 171, 737.

[14] J. W. Steed, D. R. Turner, K. J. Wallace, Core Concepts in Supramolecular Chemistry and Nanochemistry, Wiley, Chichester, UK, 2007.

[15] J. L. Atwood, J. W. Steed, Supramolecular Chemistry, 2nd ed., Chichester, UK, 2009.

[16] P. Yakovchuk, E. Protozanova, M. D. Frank-Kamenetskii, Nucleic Acids Res. 2006, 34, 564.

[17] S. Leininger, B. Olenyuk, P. J. Stang, Chem, Rev. 2000, 100, 853.

[18] C. Addicott, N. Das, P. J. Stang, Inorg. Chem. 2004, 43, 5335. 
[19] P. J. Stang, B. Olenyuk, Acc, Chem. Res. 1997, 30, 502.

[20] M. Ruben, J. Rojo, F. J. R. Salguero, L. H. Uppadine, J.-M. Lehn, Angew. Chem. Int. Ed. 2004, 43, 3644 .

[21] M. Albrecht, Chem. Rev. 2001, 101, 3457.

[22] H. Amouri, C. Desmarets, J. Moussa, Chem. Rev. 2012, 112, 2015.

[23] D. R. Turner, A. Pastor, M. Alajarin, J. W. Steed, Struct. Bond. 2004, 108, 97.

[24] B. J. Holliday, C. A. Mirkin, Angew. Chem, Int. Ed. 2001, 40, 2022.

[25] B. Olenyuk, A. Fechtenkötter, P. J. Stang, J. Chem. Soc., Dalton Trans. 1998, 1707.

[26] D. L. Caulder, K. N. Raymond, J. Chem. Soc., Dalton Trans. 1999, 1185.

[27] N. C. Gianneschi, M. S. Masar, C. A. Mirkin, Acc. Chem. Res. 2005, 38, 825.

[28] P. Stricklen, J. Verkade, J. Am. Chem. Soc. 1983, 105, 2494.

[29] M. Fujita, J. Yazaki, K. Ogura, J. Am. Chem. Soc. 1990, 112, 5645.

[30] M. Fujita, K. Ogura, Coord. Chem. Rev. 1996, 148, 249.

[31] G. F. Swiegers, T. J. Malefetse, Chem. Rev. 2000, 100, 3539.

[32] R. Chakrabarty, P. S. Mukherjee, P. J. Stang, Chem. Rev. 2011, 111, 6810.

[33] C. M. Drain, J.-M. Lehn, J. Chem. Soc., Chem. Commun.1994, 2313.

[34] F. Würthner, A. Sautter, Chem. Commun. 2000, 445.

[35] C.-C. You, F. Würthner, J. Am. Chem. Soc. 2003, 125, 9716.

[36] S. R. Seidel, P. J. Stang, Acc. Chem. Res. 2002, 35, 972.

[37] K. K. Klausmeyer, S. R. Wilson, T. B. Rauchfuss, J. Am. Chem. Soc. 1999, 121, 2705.

[38] S. C. Johannessen, R. G. Brisbois, J. P. Fischer, P. A. Grieco, A. E. Counterman, D. E. Clemmer, J. Am. Chem. Soc. 2001, 123, 3818.

[39] A. W. Maverick, F. E. Klavetter, Inorg. Chem. 1984, 23, 4129.

[40] A. W. Maverick, S. C. Buckingham, Q. Yao, J. R. Bradbury, G. G. Stanley, J. Am. Chem. Soc. 1986, 108, 7430.

[41] R. W. Saalfrank, A. Stark, K. Peters, H. G. von Schnering, Angew. Chem. Int. Ed. 1988, $27,851$.

[42] R. W. Saalfrank, A. Stark, M. Bremer, H. U. Hummel, Angew. Chem. Int. Ed. 1990, 29, 311.

[43] R. W. Saalfrank, B. Hörner, D. Stalke, J. Salbeck, Angew. Chem. Int. Ed. 1993, 32, 11791182.

[44] P. N. Baxter, J.-M. Lehn, B. O. Kneisel, G. Baum, D. Fenske, Chem. Eur. J. 1999, 5, 113.

[45] D. L. Caulder, K. N. Raymond, Acc. Chem. Res. 1999, 32, 975.

[46] D. L. Caulder, R. E. Powers, T. N. Parac, K. N. Raymond, Angew. Chem. Int. Ed. 1998, 37, 1840.

[47] D. L. Caulder, C. Brückner, R. E. Powers, S. König, T. N. Parac, J. A. Leary, K. N. Raymond, J. Am. Chem. Soc. 2001, 123, 8923.

[48] D. Fiedler, R. G. Bergman, K. N. Raymond, Angew. Chem. Int. Ed. 2004, 43, 6748.

[49] M. D. Pluth, R. G. Bergman, K. N. Raymond, Science 2007, 316, 85.

[50] M. Yoshizawa, M. Tamura, M. Fujita, J. Am. Chem. Soc. 2004, 126, 6846. 
[51] D. Fiedler, R. G. Bergman, K. N. Raymond, Angew. Chem. Int. Ed. 2006, 45, 745.

[52] V. M. Dong, D. Fiedler, B. Carl, R. G. Bergman, K. N. Raymond, J. Am. Chem. Soc. 2006, 128,14464 .

[53] M. Yoshizawa, M. Tamura, M. Fujita, Science 2006, 312, 251.

[54] D. H. Leung, R. G. Bergman, K. N. Raymond, J. Am. Chem. Soc. 2007, 129, 2746.

[55] M. L. Merlau, M. P. Mejia, S. T. Nguyen, J. T. Hupp, Angew. Chem. 2001, 113, 4369.

[56] C. G. Oliveri, P. A. Ulmann, M. J. Wiester, C. A. Mirkin, Acc Chem Res 2008, 41, 16181629.

[57] J. C. Jeffrey, T. B. Rauchfuss, Inorg. Chem. 1979, 18, 2658.

[58] A. Bader, E. Lindner, Coord. Chem. Rev. 1991, 108, 27.

[59] J. R. Farrell, C. A. Mirkin, L. M. Liable-Sands, A. L. Rheingold, J. Am. Chem. Soc. 1998, 120, 11834.

[60] C. Piguet, G. Bernardinelli, G. Hopfgartner, Chem. Rev. 1997, 97, 2005.

[61] M. Han, D. M. Engelhard, G. H. Clever, Chem. Soc. Rev. 2014, 43, 1848.

[62] M. Fujita, N. Fujita, K. Ogura, K. Yamaguchi, Nature 1999, 400, 52

[63] A. Westcott, J. Fisher, L. P. Harding, P. Rizkallah, M. J. Hardie, J. Am. Chem. Soc. 2008, 130, 2950.

[64] J. J. Henkelis, T. K. Ronson, L. P. Harding, M. J. Hardie, Chem. Commun. 2011, 47, 6560.

[65] S. Freye, J. Hey, A. T. Galán, D. Stalke, R. H. Irmer, M. John, G. H. Clever, Angew. Chem. Int. Ed. 2012, 51, 2191.

[66] S. Freye, D. M. Engelhard, M. John, G. H. Clever, Chem. Eur. J. 2013, 19, 2114.

[67] S. Freye, R. Michel, D. Stalke, M. Pawliczek, H. Frauendorf, G. H. Clever, J. Am. Chem. Soc. 2013, 135, 8476.

[68] M. Frank, J. Hey, I. Balcioglu, Y. S. Chen, D. Stalke, T. Suenobu, S. Fukuzumi, H. Frauendorf, G. H. Clever, Angew. Chem. Int. Ed. 2013, 52, 10102.

[69] M. Frank, L. Krause, R. H. Irmer, D. Stalke, G. H. Clever, Dalton Trans. 2014, 43, 4587.

[70] R. Zhu, J. Lübben, B. Dittrich, G. H. Clever, Angew. Chem. Int. Ed. 2015, 54, 2796.

[71] M. Han, J. Hey, W. Kawamura, D. Stalke, M. Shionoya, G. H. Clever, Inorg. Chem. 2012, $51,9574$.

[72] G. H. Clever, S. Tashiro, M. Shionoya, Angew. Chem. Int. Ed. 2009, 48, 7010.

[73] D. M. Engelhard, S. Freye, K. Grohe, M. John, G. H. Clever, Angew. Chem. Int. Ed. 2012, 51,4747 .

[74] S. Hiraoka, Y. Sakata, M. Shionoya, J. Am. Chem. Soc. 2008, 130, 10058.

[75] M. Wang, V. Vajpayee, S. Shanmugaraju, Y.-R. Zheng, Z. Zhao, H. Kim, P. S. Mukherjee, K.-W. Chi, P. J. Stang, Inorg. Chem. 2011, 50, 10640.

[76] Y.-R. Zheng, W.-J. Lan, M. Wang, T. R. Cook, P. J. Stang, J. Am. Chem. Soc. 2011, 133, 17045.

[77] M. Wang, Y.-R. Zheng, T. R. Cook, P. J. Stang, Inorg. Chem. 2011, 50, 6107.

[78] Y. Fang, T. Murase, S. Sato, M. Fujita, J. Am. Chem. Soc. 2013, 135, 613.

[79] M. L. Saha, M. Schmittel, J. Am. Chem. Soc. 2013, 135, 17743.

[80] S. Durot, J. Taesch, V. Heitz, Chem. Rev. 2014, 114, 8542. 
[81] M. M. J. Smulders, I. A. Riddell, C. Browne, J. R. Nitschke, Chem. Soc. Rev. 2013, 42, 1728.

[82] P. N. Baxter, J--M. Lehn, G. Baum, D. Fenske, Chem. Eur. J. 1999, 5, 102.

[83] T. Nakamura, H. Ube, M. Shiro, M. Shionoya, Angew. Chem. Int. Ed. 2013, 52, 720.

[84] M. Han, R. Michel, G. H. Clever, Chem. Eur. J. 2014, 20, 10640.

[85] N. Ahmad, H. A. Younus, A. H. Chughtai, F. Verpoort, Chem. Soc. Rev. 2015, 44, 9.

[86] M. Iyoda, J. Yamakawa, M. J. Rahman, Angew. Chem. Int. Ed. 2011, 50, 10522.

[87] M. Fujita, S.-Y. Yu, T. Kusukawa, H. Funaki, K. Ogura, K. Yamaguchi, Angew. Chem. Int. Ed. 1998, 37, 2082.

[88] M. Yoshizawa, M. Tamura, M. Fujita, Angew. Chem. Int. Ed. 2007, 46, 3874.

[89] K. Omoto, S. Tashiro, M. Kuritani, M. Shionoya, J. Am. Chem. Soc. 2014, 136, 17946.

[90] B. Therrien, Eur. J. Inorg. Chem. 2009, 2009, 2445.

[91] Y.-F. Han, Y.-J. Lin, T. A. Hor, G.-X. Jin, Organometallics 2012, 31, 995.

[92] Y. Yamauchi, M. Yoshizawa, M. Fujita, J. Am. Chem. Soc. 2008, 130, 5832.

[93] S. K. Samanta, O. V. Singh, R. K. Jain, Trends Biotechnol. 2002, 20, 243.

[94] M. Ziegler, J. L. Brumaghim, K. N. Raymond, Angew. Chem. Int. Ed. 2000, 39, 4119.

[95] T. Sawada, M. Yoshizawa, S. Sato, M. Fujita, Nat. Chem. 2009, 1, 53.

[96] P. Mal, B. Breiner, K. Rissanen, J. R. Nitschke, Science 2009, 324, 1697.

[97] I. A. Riddell, M. M. J. Smulders, J. K. Clegg, J. R. Nitschke, Chem. Commun. 2011, 47, 457.

[98] Y. Liu, X. Wu, C. He, Z. Li, C. Duan, Dalton Trans. 2010, 39, 7727.

[99] A. M. Johnson, O. Moshe, A. S. Gamboa, B. W. Langloss, J. F. K. Limtiaco, C. K. Larive, R. J. Hooley, Inorg. Chem. 2011, 50, 9430.

[100] B. K. McMahon, T. Gunnlaugsson, J. Am. Chem. Soc. 2012, 134, 10725.

[101] J. P. Ma, S. Q. Wang, C. W. Zhao, H. Y. Wang, Y. B. Dong, Chem. Commun. 2014, 50, 4721.

[102] C. Schouwey, R. Scopelliti, K. Severin, Chem. E. J. 2013, 19, 6274.

[103] K.-I. Yamashita, M. Kawano, M. Fujita, Chem, Commun. 2007, 4102.

[104] M. Yoshizawa, J. K. Klosterman, M. Fujita, Angew. Chem. Int. Ed. 2009, 48, 3418.

[105] S. Zarra, D. M. Wood, D. A. Roberts, J. R. Nitschke, Chem. Soc. Rev. 2015, 44, 419.

[106] M. Fujita, D. Oguro, M. Miyazawa, H. Oka, K. Yamaguchi, K. Ogura, Nature 1995, $378,469$.

[107] P. V. R. Schleyer, M. Manoharan, H. Jiao, F. Stahl, Org. Lett. 2001, 3, 3643.

[108] L. R. MacGillivray, J. L. Atwood, Nature 1997, 389, 469.

[109] Q. Zhang, K. Tiefenbacher, Nat. Chem. 2015, 7, 197.

[110] C. García-Simón, R. G. Doria, S. Raoufmoghaddam, T. Parella, M. Costas, X. Ribas, J. N. H. Reek, J. Am. Chem. Soc. 2015, 137, 2680.

[111] M. J. Hannon, Chem. Soc. Rev. 2007, 36, 280.

[112] S. Tashiro, M. Tominaga, M. Kawano, B. Therrien, T. Ozeki, M. Fujita, , J. Am. Chem. Soc. 2005, 127, 4546. 
[113] D. Fujita, K. Suzuki, S. Sato, M. Yagi-Utsumi, Y. Yamaguchi, N. Mizuno, T. Kumasaka, M. Takata, M. Noda, S. Uchiyama, K. Kato, M. Fujita, Nat. Commun. 2012, 3, 1093.

[114] Y. Hatakeyama, T. Sawada, M. Kawano, M. Fujita, Angew. Chem. Int. Ed. 2009, 48, 8695.

[115] B. Therrien, G. Süss-Fink, P. Govindaswamy, A. K. Renfrew, P. J. Dyson, Angew. Chem. Int. Ed. 2008, 47, 3773.

[116] J. Mattsson, P. Govindaswamy, J. Furrer, Y. Sei, K. Yamaguchi, G. Süss-Fink, B. Therrien, Organometallics 2008, 27, 4346.

[117] B. Therrien, Chem. Eur. J. 2013, 19, 8378.

[118] J. E. M. Lewis, E. L. Gavey, S. A. Cameron, J. D. Crowley, Chem. Sci. 2012, 3, 778.

[119] W. Cullen, S. Turega, C. A. Hunter, M. D. Ward, Chem. Sci. 2015, 6, 625.

[120] G. Mayer, A. Heckel, Angew. Chem. Int. Ed. 2006, 45, 4900.

[121] B. L. Feringa, R. A. van Delden, N. Koumura, E. M. Geertsema, Chem. Rev. 2000, 100, 1789.

[122] B. L. B. Feringa, Acc. Chem. Res. 2001, 34, 504.

[123] M. Irie, Chem. Rev. 2000, 100, 1685.

[124] A. Peters, N. R. Branda, J. Am. Chem. Soc. 2003, 125, 3404.

[125] J. W. Canary, Chem. Soc. Rev. 2009, 38, 747.

[126] F. M. Raymo, S. Giordani, J. Am. Chem. Soc. 2001, 123, 4651.

[122] B. L. B. Feringa, Acc. Chem. Res. 2001, 34, 504.

[127] B. L. Feringa, R. A. van Delden, M.K.J. ter Wiel, in Molecular Switches, Chapter 5 (Ed.: B. L. Feringa), Wiley-VCH, Weinheim, 2001.

[128] D.-H. Qu, Q.-C. Wang, Q.-W. Zhang, X. Ma, H. Tian, Chem. Rev. 2015, 115, 7543.

[129] M. Hardouin-Lerouge, P. Hudhomme, M. Salle, Chem. Soc. Rev. 2011, 40, 30.

[130] V. Valderrey, G. Aragay, P. Ballester, Coord. Chem. Rev. 2014, 258-259, 137.

[131] T. Muraoka, K. Kinbara, Y. Kobayashi, T. Aida, J. Am. Chem. Soc. 2003, 125, 5612.

[132] E. R. Kay, D. A. Leigh, F. Zerbetto, Angew. Chem. Int. Ed. 2007, 46, 72.

[133] A. Ueno, H. Yoshimura, R. Saka, T. Osa, J. Am. Chem. Soc. 1979, 101, 2779.

[134] R. Rojanathanes, T. Tuntulani, W. Bhanthumnavin, M. Sukwattanasinitt, Org. Lett. 2005, 7, 3401.

[135] T. Murase, S. Sato, M. Fujita, Angew. Chem. Int. Ed. 2007, 46, 5133.

[136] N. Iwasawa, H. Takahagi, K. Ono, K. Fujii, H. Uekusa, Chem. Commun. 2012, 48, 7477.

[137] J. Park, L.-B. Sun, Y.-P. Chen, Z. Perry, H.-C. Zhou, Angew. Chem. Int. Ed. 2014, 53, 5842.

[138] H. Dube, D. Ajami, J. Rebek, Jr., Angew. Chem. Int. Ed. 2010, 49, 3192.

[139] G. H. Clever, W. Kawamura, M. Shionoya, Inorg. Chem. 2011, 50, 4689.

[140] G. H. Clever, S. Tashiro, M. Shionoya, J. Am. Chem. Soc. 2010, 132, 9973.

[141] M. Fukuda, R. Sekiya, R. Kuroda, Angew. Chem. Int. Ed. 2008, 47, 706. 


\section{OBJECTIVES}

The field of supramolecular chemistry has been growing rapidly over the past decades. Although interesting new developments have been described in the literature, there is still much room for new discoveries to be made. The aims of this thesis are outlined below:

- To encapsulate a guest with functionality inside a coordination cage

Many host-guest systems make use of simple guest molecules for studying the underlying non-covalent interactions. ${ }^{[1]}$ Host-guest systems, however, are progressing from simple binding towards specific functionality. In this regard, the encapsulation of functional guests, such as polyoxometalates (POMs), which have been widely used in the field of catalysis,[5] medicine, ${ }^{[3]}$ material science ${ }^{[4]}$ and host-guest chemistry, ${ }^{[5-7]}$ has attracted growing attention. However, complete encapsulation of such an anionic guest inside a discrete host is rare ${ }^{[7,8]}$ and therefore worthy of further study. For this, a previously reported $\mathrm{Pd}_{2} \mathbf{L}_{4}$ coordination cage[9] (Figure 2-1) will be selected and tested for its suitability to encapsulate the hexamolybdate hexamolybdate $\left[\mathrm{Mo}_{6} \mathrm{O}_{19}\right]^{2-}$ anion. The encapsulation of the prototypical Lindqvist-type compound inside a discrete host may provide the possibility of modulating the cluster's electronic and optical properties and the accessibility of substrates to the POM core in catalytically active systems by the design of the surrounding cage structure (Chapter 3 ). 


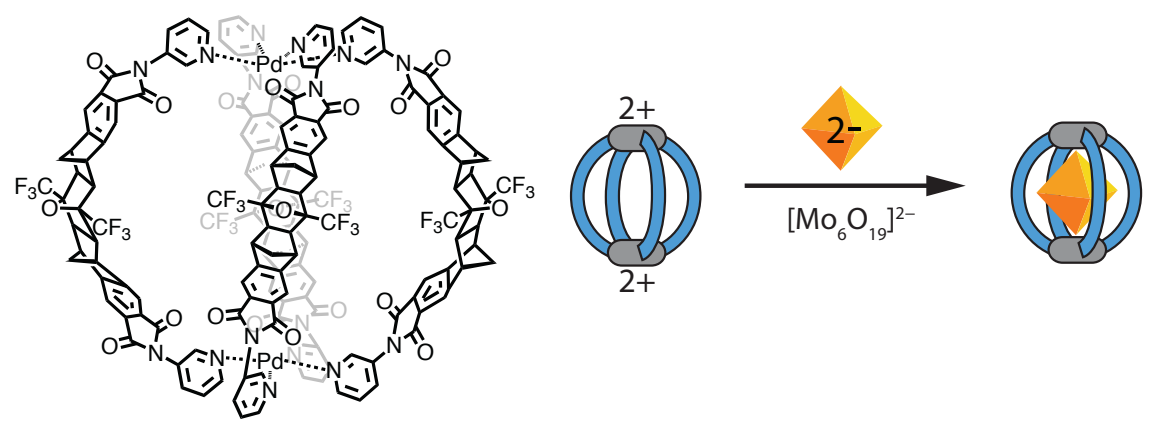

Figure 2-1 Chemical structure of previously reported $\operatorname{Pd}_{2} \mathbf{L}_{4}$ cage (left) and the schematic representation of the predicted uptake of an anionic polyoxometallate (right).

- To synthesize self-assembled cage with lower symmetry

Studies in developing self-assembled molecules with lower symmetry have aroused interest because most of the large discrete self-assemblies reported so far are highly symmetric polyhedra.[10] Only a few low symmetric self-assemblies have been reported which make use of either heteroleptic coordination at metal centres ${ }^{[11,12]}$ or more than one type of metal.[13] Therefore, to design a homonuclear self-assembled systems with lower symmetry which uses homoleptic coordination ${ }^{[14]}$ presents a challenge. To achieve such a structure possessing low symmetry, the design of a tripodal tris-pyridyl ligand with the appropriate shape and donor sites will be carried out (Figure 2-2a). Based on the mathematical and geometric analysis (Figure 2-2b), the most synthetically feasible ligand will be selected and synthesized for subsequent cage formation. The host-guest chemistry will be studied on account of the accomplishment of the cuboid cage, as low symmetric self-assembled structures can be expected to show enhanced selectivity towards guests with irregular structures (Chapter 4).

a)

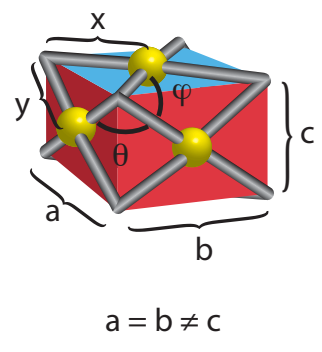

b)
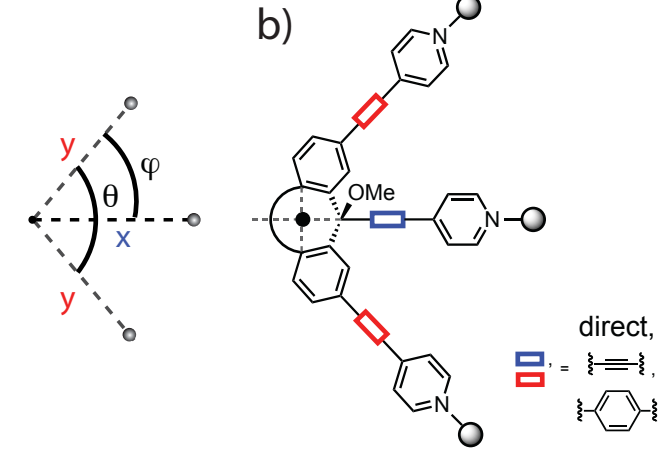

Figure 2-2 ) Schematic model of a desired square-cuboid cage composed of carefully designed tripodal ligands. b) Synthetically plausible ligand structures for computational screening after taking into consideration the mathematical and geometric analysis. 
- To introduce light-switchable functionality into a self-assembly cage system

Apart from the interest directed at the encapsulation of functional guests and reduction of structural symmetry as outlined above, introduction of switchable features into supramolecular host system is particularly appealing. Light-switchable functionalities are extremely attractive because of the convenience of operating with light.[15] Lightswitchable coordination cages, however, have been rarely described[16] among the intensively developed light-switchable moieties implemented in supramolecular structures.[17-21] Two of the most studied photoswitches ${ }^{[22]}$ were considered in this work: azobenzenes and diarylethenes. From previous experience with azobenzene in our research group,[23] diarylethene was chosen as the ligand backbone, because the lightinduced structural changes are more predictable (small geometry change upon irradiation) and efficient with higher conversion. ${ }^{[22]}$ Ligand $\mathbf{L}^{1}$ will be synthesized and utilized for cage formation (Figure 2-3a). One of the important issues regarding the targeted cage is, whether the cage structure can be influenced upon irradiation without disassembly of the system. Besides the structural conversion of the cage, investigation of the relationship between cage switching and guest binding is of great importance (Chapter 5).

a)

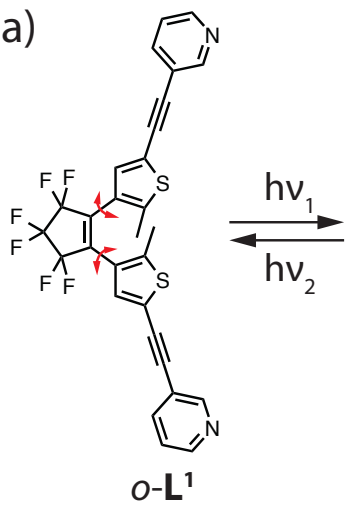

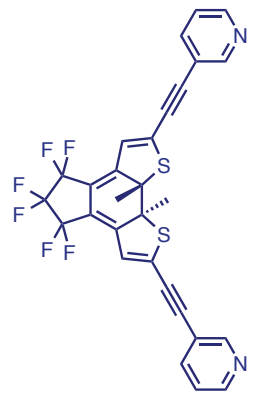

$c-\mathbf{L}^{1}$ b)

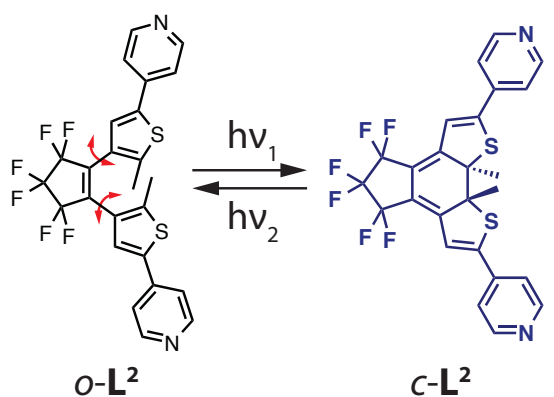

Figure 2-3 Aimed chemical structures of the light-switchable ligand a) $\mathbf{L}^{\mathbf{1}}$ b) $\mathbf{L}^{2}$ and their light-induced switching.

Furthermore, inspired by the successful empirical prediction of large polyhedra formation developed by Fujita,[24] another ligand $\mathbf{L}^{2}$ based on the same DTE backbone, carrying two para-substituted pyridyl donors (Figure 2-3b) will be projected to be synthesised[25] and employed in cage formation. The open- and closed-form ligand is expected to behave quite different upon complexation with $\mathrm{Pd}^{\text {II }}$ due to the different ligand substitution and therefore bend angle. The switching processes between the ligands and self-assemblies are going to be investigated. In addition, the kinetics of the photoconversion between free ligand $\mathbf{L}^{1}$ and $\mathbf{L}^{2}$ and their palladium-mediated open- and closed-form assemblies will be 
compared in order to get a better understanding of how the irradiation affects the cage assemblies.

[1] H. Amouri, C. Desmarets, J. Moussa, Chem. Rev. 2012, 112, 2015.

[2] S.-S. Wang, G.-Y. Yang, Chem. Rev. 2015, 115, 4893.

[3] D.-L. Long, R. Tsunashima, L. Cronin, Angew. Chem. Int. Ed. 2010, 49, 1736.

[4] A. Dolbecq, E. Dumas, C. R. Mayer, P. Mialane, Chem. Rev. 2010, 110, 6009.

[5] J. M. Knaust, C. Inman, S. W. Keller, Chem. Commun. 2004, 492.

[6] T. Chatterjee, M. Sarma, S. K. Das, Cryst. Growth Des. 2010, 10, 3149.

[7] X. Kuang, X.-Y. Wu, J. Zhang, C.-Z. Lu, Chem. Commun. 2011, 4150.

[8] Y. Liu, C. Hu, A. Comotti, M. D. Ward, Science 2011, 333, 436.

[9] G. H. Clever, S. Tashiro, M. Shionoya, Angew. Chem. Int. Ed. 2009, 48, 7010.

[10] T. R. Cook, Y.-R. Zheng, P. J. Stang, Chem. Rev. 2013, 113, 734.

[11] Y.-R. Zheng, Z. Zhao, M. Wang, K. Ghosh, J. B. Pollock, T. R. Cook, P. J. Stang, J. Am. Chem. Soc. 2010, 132, 16873.

[12] K. Kumazawa, K. Biradha, T. Kusukawa, T. Okano, M. Fujita, Angew. Chem. Int. Ed. 2003, 42, 3909.

[13] S. Hiraoka, Y. Sakata, M. Shionoya, J. Am. Chem. Soc. 2008, 130, 10058.

[14] Q.-F. Sun, S. Sato, M. Fujita, Nat. Chem. 2012, 4, 330.

[15] G. Mayer, A. Heckel, Angew. Chem. Int. Ed. 2006, 45, 4900.

[16] T. Murase, S. Sato, M. Fujita, Angew. Chem. Int. Ed. 2007, 46, 5133.

[17] M. Blank, L. Soo, N. Wassermann, B. Erlanger, Science 1981, 214, 70.

[18] M. Irie, M. Kato, J. Am. Chem. Soc. 1985, 107, 1024.

[19] N. Iwasawa, H. Takahagi, K. Ono, K. Fujii, H. Uekusa, Chem. Commun. 2012, 48, 7477.

[20] S. Chen, L. J. Chen, H.-B. Yang, H. Tian, W. Zhu, J. Am. Chem. Soc. 2012, 134, 13596.

[21] H. Kai, S. Nara, K. Kinbara, T. Aida, J. Am. Chem. Soc. 2008, 130, 6725.

[22] B. L. Feringa, W. R. Browne, Eds. Molecular Switches, 2011, Wiley-VCH, Weinheim.

[23] D. Engelhard, Master Thesis, 2011, The Clever Lab, Georg-August-Universität Göttingen.

[24] J. Bunzen, J. Iwasa, P. Bonakdarzadeh, E. Numata, K. Rissanen, S. Sato, M. Fujita, Angew. Chem. Int. Ed. 2012, 51, 3161.

[25] H. Miyasaka, S. Araki, A. Tabata, T. Nobuto, N. Malaga, M. Irie, Chem. Phys. Letters, 1994, 230, 249. 


\section{AN INCLUSION COMPLEX OF HEXAMOLYBDATE INSIDE A SUPRAMOLECULAR CAGE ${ }^{1}$}

In this chapter, the encapsulation of a spherical dianionic hexamolybdate $\left[\mathrm{Mo}_{6} \mathrm{O}_{19}\right]^{2-}$ guest molecule in a $\mathrm{Pd}_{2} \mathrm{~L}_{4}$ self-assembled cage compound is described. Interestingly, a structure conversion was observed by recrystallization after an excess of hexamolybdate guest was added to the 1:1 inclusion complex. The strategies for the formation of the inclusion complex and the compound after structure conversion are explained and comprehensively characterized both in solution and in the solid state.

\subsection{Introduction}

Polyoxometalates (POMs) are discrete nanosized anionic metal-oxide clusters. They show a great diversity of sizes, shapes, and nuclearities,[1] which have attracted considerable attention in various areas ranging from of medicine,[2] catalysis,[3] material[4] and surface science[5] because of their intriguing structural, electrochemical and photophysical properties.[6,7] The huge number of polyoxometalates can be classified in three subsets (Figure 3-1).[8] (i) Heteropolyanions, the most developed subset so far, are robust metal-

\footnotetext{
1 The work presented in this chapter has been published: M. Han, J. Hey, W. Kawamura, D. Stalke, M. Shionoya, G. H. Clever, Inorg. Chem. 2012, 51, 9574.
} 
oxide clusters containing $\mathrm{PO}_{4}{ }^{3-}$, and $\mathrm{SO}_{4}{ }^{2-}$ heteroanions, which are widely used in catalysis research. (ii) In contrast, metal-oxide clusters without internal heteroatoms are defined as isopolyanions. Although less stable than the heteropolyanionic frameworks, they are still considered as important building blocks, due to their physical properties, such as strongly basic oxygen surface and high charges. (iii) Reduced $\mathrm{Mo}^{\mathrm{VI}}$ and $\mathrm{Mo}^{\mathrm{V}}$ mixed oxide clusters (reduced Mo-blue and Mo-brown), such as $\left\{\mathrm{Mo}_{154}\right\}$ ring-, ${ }^{[9]}\left\{\mathrm{Mo}_{132}\right\}$ sphere-,[10] and $\left\{\mathrm{Mo}_{368}\right\}$ hedgehog-shaped[11] clusters. This reveals that the cluster size expansion might be favored under strong reducing condition, which means the more $\mathrm{Mo}^{\mathrm{V}}$ involved in the framework, the larger the whole POM systems are.

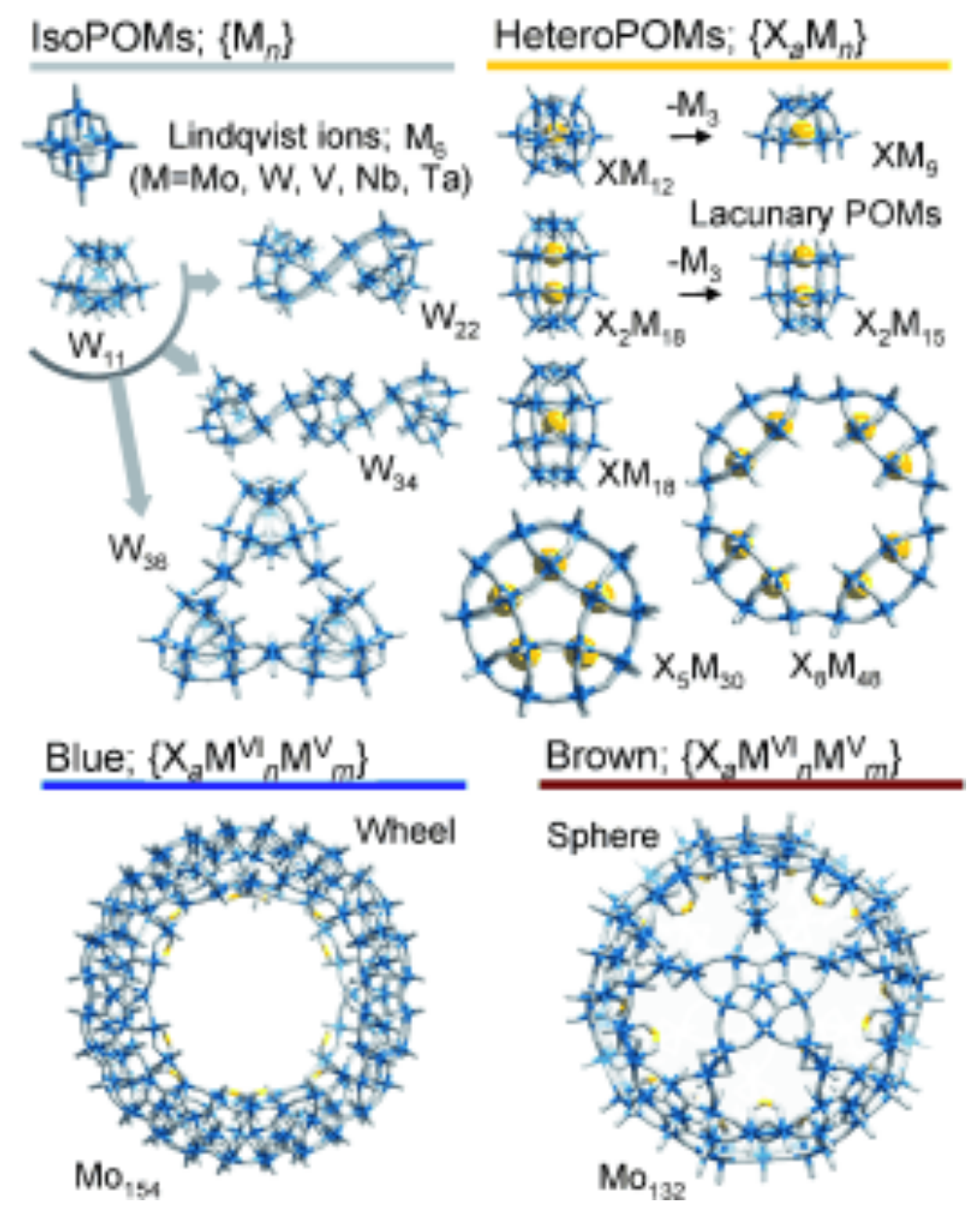

Figure 3-1 Overview of the polyoxometalates subsets. (Metal atoms: blue; 0: gray; heteroatoms: yellow). Copyright (C) 2010 WILEY-VCH Verlag GmbH \& Co.[8]

The preparation of POMs is therefore an active and attractive field of research. In particular the combination of polyoxometalates with covalent organic functionalities has given rise to a variety of hybrid compounds, ${ }^{[12-17]}$ such as giant wheels, ${ }^{[9]}$ cages and capsules,[18,19] heteronuclear clusters,[20,21] dendrimers,[22] and extended framework 
materials,[23,24] which show promising properties for guest uptake,[25,26] gel formation,[27] and light-induced electron transfer.[28]

Moreover, hybrid organic-inorganic rotaxanes and molecular shuttles consisting of heterometallic POM rings were reported by Winpenny and co-workers. [29] Zhang et al. introduced nanoscale POM-organic hybrid chiral molecular rods ${ }^{[30]}$ that were constructed from both Lindquist and Anderson POMs. Later on, Fang et al. described the first hybrid complexes based on cucurbit[n]uril macrocycles and inorganic redox-active clusters.[31] Meanwhile, POMs have been used as redox-active network in frameworks hosting ascorbic acid,[23] and helping the crystallization of cationic crown ethers in host-guest chemistry serving as large counteranions. ${ }^{[32]}$ Furthermore, POMs have been applied to encapsulate guests inside a metal-organic pseudorotaxane framework,[33] and associated with bowlshaped cyclotriveratrylenes. ${ }^{[34]}$ However, there are few reports on the full encapsulation of a POM anion inside a discrete host compound.[35-37]

Previously reported coordination cage $\mathbf{1}^{[38]}$ (Figure 3-2d) containing four concave, rigid bis-monodentate pyridyl ligands and two square-planar coordinated $\mathrm{Pd}^{\mathrm{II}}$ or $\mathrm{Pt}^{\mathrm{II}}$ ions, was considered as a suitable host molecule since its hollow cavity is large enough to encapsulate a variety of guests such as aromatic disulfonates, ${ }^{[39]}$ which was shown to lead to an attractive light-triggered crystallization process when a light-switchable guest was involved.[40] Additional to this, pseudorotaxanes have been achieved by interlocking the cage compound $\mathbf{1}$ with bis-sulfonate axles.[41] In a separate study, the cage compound $\mathbf{1}$ was also shown to coencapsulate alternately stacked anionic and cationic square-planar platinum ${ }^{\text {II }}$ complexes. ${ }^{[42]}$ 


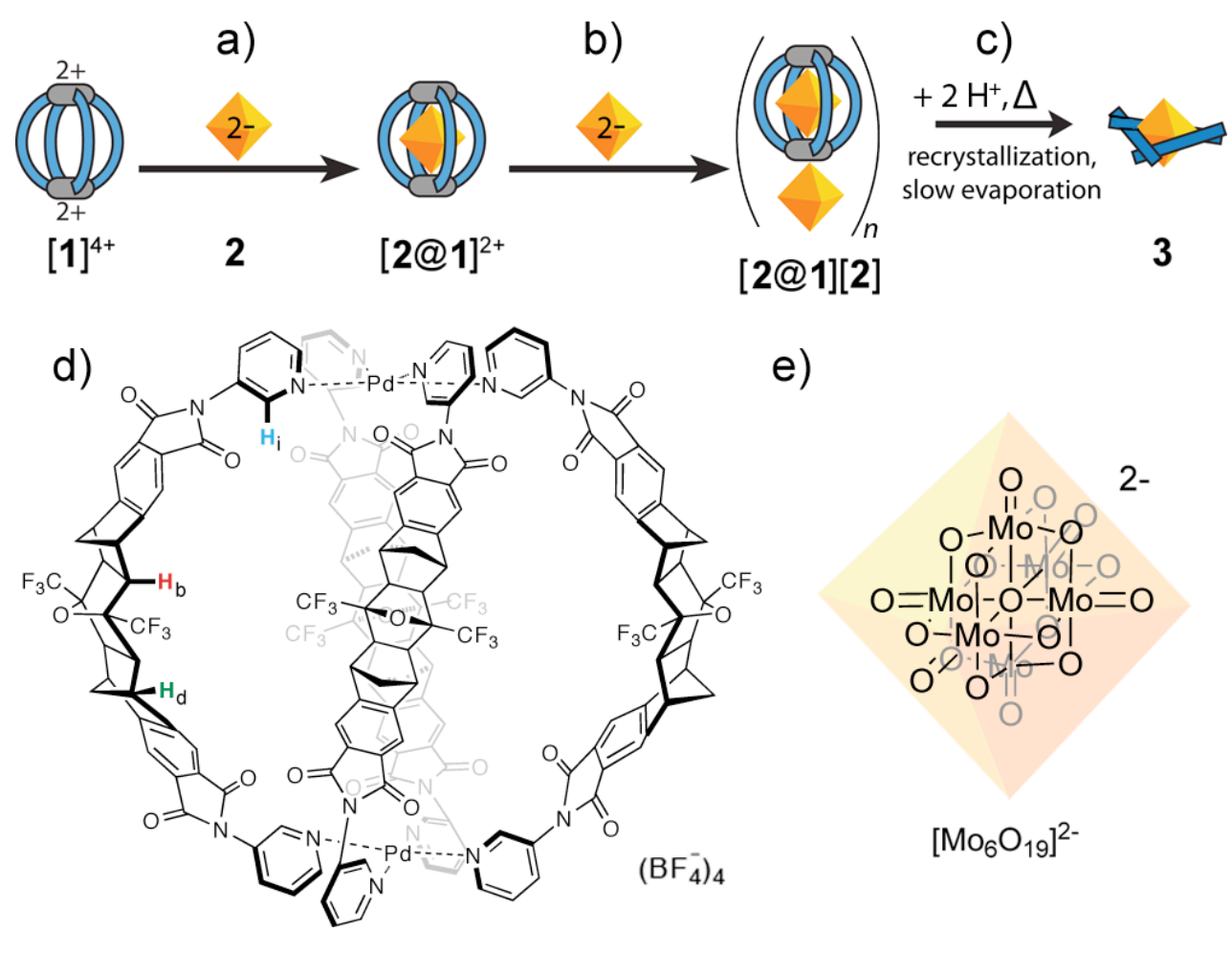

Figure 3-2 Strategy of the formation of (a) a soluble inclusion complex [2@1 $]^{2+}$ and (b) the precipitation [2@1][2] upon the addition of a second equivalent of 2. (c) Conversion upon recrystallization into the adduct $\left.\left\{\left[\mathrm{Mo}_{6} \mathrm{O}_{19}\right]^{2-@(l i g a n d)}\right)_{3}+2 \mathrm{H}^{+}\right\} 3$. Chemical structures of (d) cage 1 and (e) anionic hexamolybdate 2. Copyright (C) 2012, American Chemical Society.

\subsection{Formation of host-guest complex [2@1 $]^{2+}$}

The previous systematic study of the host-guest interactions of the coordination cage $\mathbf{1}$ revealed the host's ability to encapsulate of guests with suitable size and charge. The octahedral hexamolybdate $\left[\mathrm{Mo}_{6} \mathrm{O}_{19}\right]^{2-} 2$ (Figure 3-2e) with a spherical delocalized negative charge, was selected in order to further probe the binding properties of the coordination cage $\mathbf{1}$.

In the master thesis of Wataru Kawamura, the NMR spectroscopic titration was carried out.[54] Formation of inclusion complex [2@1 $]^{2+}$ was determined by ${ }^{1} \mathrm{H}$ NMR titration of guest $\left({ }^{n} \mathrm{Bu}_{4} \mathrm{~N}\right)_{2}\left[\mathrm{Mo}_{6} \mathrm{O}_{19}\right]$, tetra- $n$-butylammonium salt of 2 , into a $0.7 \mathrm{mM}$ acetonitrile solution of cage 1 . The guest solution 2 was added stepwise, in a sequence of $0.25,0.5$, $0.75,1,00,1.50,2.00$ equivalents (Figure 3-3a-g). Interestingly, the signal assigned the endo norbornene backbone proton $\mathrm{H}_{\mathrm{b}}$ (red in Figure 3-3), which pointed inside to the cage cavity, exhibits a downfield shift upon addition of 1.0 equivalent of guest 2 . A slight upfield shift of the $\mathrm{H}_{\mathrm{d}}$ proton (green in Figure 3-3) in 1 was also observed. After the addition of the first equivalent of guest 2 , no further signal shifts of both protons $\mathrm{H}_{\mathrm{b}}$ and $\mathrm{H}_{\mathrm{d}}$ 
were observed, which could be clearly seen from the plotting of $\Delta \delta$ against the added equivalents of 2 (Figure 3-3h), suggesting that the all cages were saturated with guest 2 after the addition 1 equivalent. In particular, no significant shift of the inward pointing pyridine proton $\mathrm{H}_{\mathrm{i}}$ (blue in Figure 3-3), which is located adjacent to the $\mathrm{Pd}^{\mathrm{II}}$ ions, was observed during the addition of guest 2 .

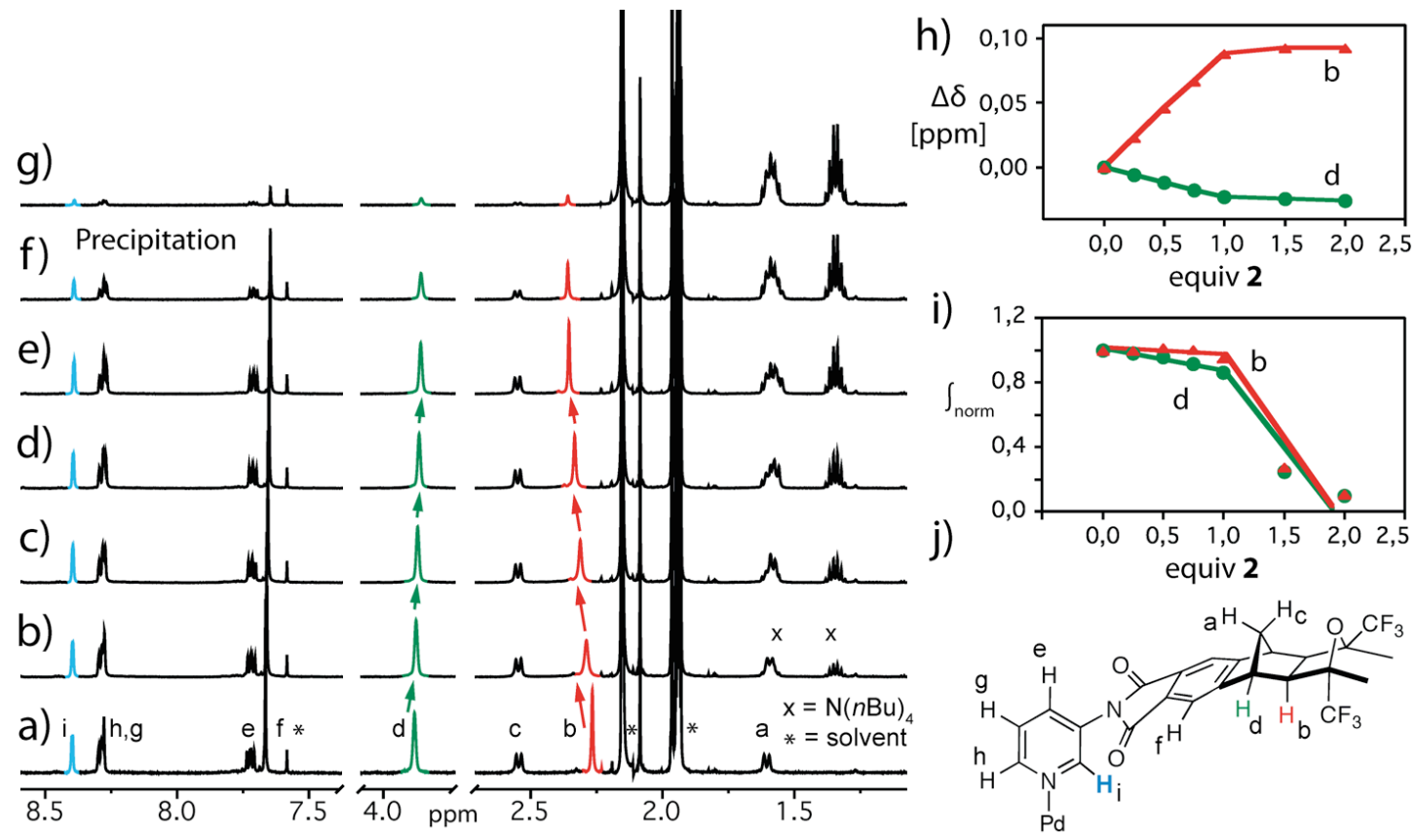

Figure 3-3 (a)-(g) ${ }^{1} \mathrm{H}$ NMR titration $\left(500 \mathrm{MHz}, \mathrm{CD}_{3} \mathrm{CN}, 298 \mathrm{~K}\right)$ of a solution of cage 1 in $\mathrm{CD}_{3} \mathrm{CN}$ with increasing equivalents of guest $2\left({ }^{n} \mathrm{Bu}_{4} \mathrm{~N}^{+}\right.$signals at 0.9 and $3.1 \mathrm{ppm}$ omitted). The plotting of (h) the relative change of chemical shift $\Delta \delta$ and (i) the relative integral values of the protons $\mathrm{H}_{\mathrm{b}}$ and $\mathrm{H}_{\mathrm{d}}$ shows the sharp transition between guest uptake and guest-induced precipitation after the addition of 1 equivalent of 2. (j) Signal assignment to the structure of cage $1 .{ }^{2}$ Copyright (C) 2012, American Chemical Society.

To gain further insight into the structure, a semi-empirical structure optimization of the inclusion complex [2@1 $]^{2+}$ was performed (Figure 3-4). Molecular simulation indicated that the shortest distance between the inward pointing pyridine proton $\mathrm{H}_{\mathrm{i}}$ and a terminal oxygen atom of guest 2 is $4.40 \AA$, and the shortest distance between the ligand backbone proton $\mathrm{H}_{\mathrm{b}}$ and another terminal oxygen atom of $\mathbf{2}$ is $2.04 \AA$ (Figure $\mathbf{3 - 4 b}$.). This comparison of the two distances supports the signal shift tendencies observed for protons $\mathrm{H}_{\mathrm{i}}$ and $\mathrm{H}_{\mathrm{b}}$. The spherical hexamolybdate guest molecule is much closer to the $\mathrm{H}_{\mathrm{b}}$ atoms of

2 NMR titration was recorded by Wataru Kawamura. Shionoya Lab, Tokyo University. 
the norbornene backbone than the $\mathrm{H}_{\mathrm{i}}$ of the pyridine binding site, which had a strong effect on $\mathrm{H}_{\mathrm{b}}$, and thus resulted in a stronger downfield shift. The shift of the proton $\mathrm{H}_{\mathrm{i}}$ signal could barely be observed during the encapsulation process, which is different from previously published work. ${ }^{[39]}$ The signal of $\mathrm{H}_{\mathrm{i}}$ always undergoes a remarkable downfield shift when the cage $\mathbf{1}$ encapsulates dianionic aromatic guests, such as 2,6-naphthyl disulfonate. ${ }^{[39]}$ As the sulfonate groups of the rod-like guests are oriented more or less directly towards to the hydrogen atom $\mathrm{H}_{\mathrm{i}}$ of the cage, this results in a notable signal shift of $\mathrm{H}_{\mathrm{i}}$
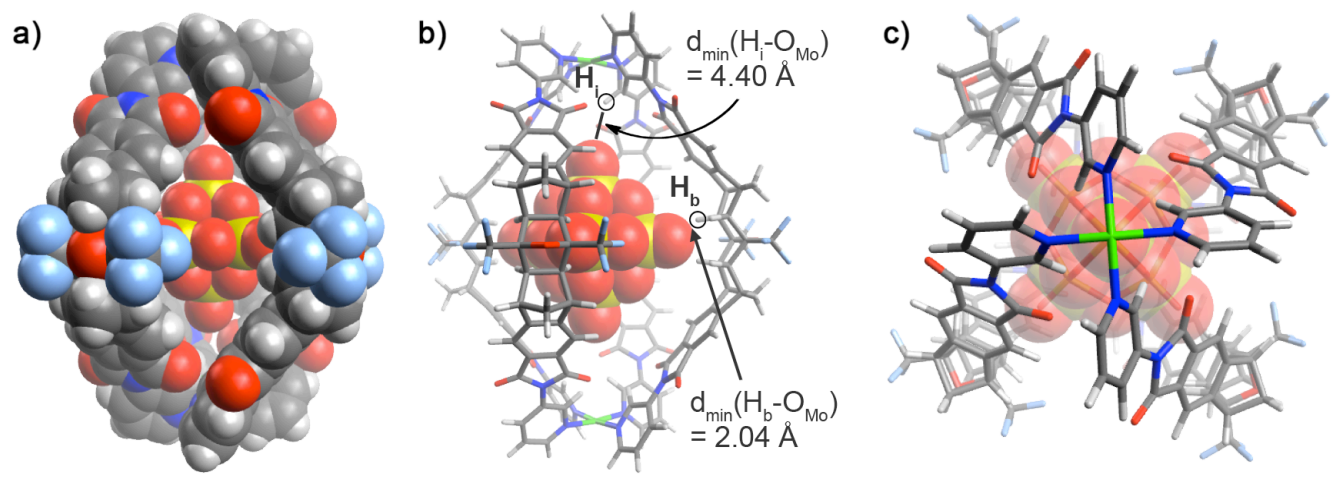

Figure 3-4 PM6 optimized structure of the host-guest complex [2@1 ${ }^{2+}$. a) Space-filling view showing guest $\mathbf{2}$ encapsulated in $\mathbf{1}$ with b) highlighting the minimum distances between protons $\mathrm{H}_{\mathrm{i}}$ and $\mathrm{H}_{\mathrm{b}}$ with oxygen atom of $\mathbf{2}$. c) Top view of [2@1 $]^{2+}$.

Electrospray ionization (ESI) mass spectrometry analysis was performed in order to provide further evidence of the 1:1 formation of host-guest complex (Figure 3-5). In the mass spectrum of [2@1] $\left(\mathrm{BF}_{4}\right)_{2}$, three prominent peaks at $m / z=2055.4,807.7$, and 755.3 were assigned to $[\mathbf{2} @ \mathbf{1}]^{2+},[\mathbf{1}]^{4+}$ and $[\text { Ligand }+\mathrm{H}]^{+}$, respectively.

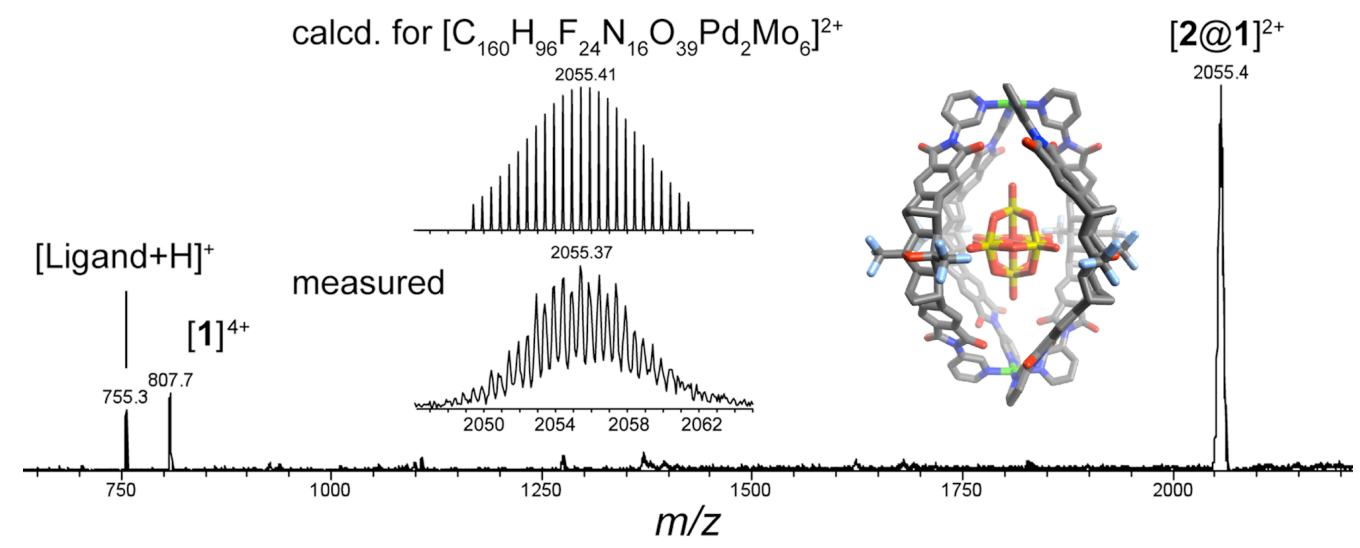

Figure 3-5 ESI-MS spectrum of the host-guest complex [2@1] $\left(\mathrm{BF}_{4}\right)_{2}$ (inset: corresponding PM6 optimized structure). Copyright (c) 2012, American Chemical Society. 


\subsection{Palladium-free assembly}

Once the 1:1 host-guest complex [2@1 $]^{2+}$ was formed, further addition of guest $\mathbf{2}$ leads immediately to a precipitation, which could be due to the formation of a [2@1][2] insoluble salt. The aggregation caused a slow intensity decrease of the proton signals in ${ }^{1} \mathrm{H}$ NMR spectra. The cage signals disappeared completely after 2 equivalents of guest 2 were added to the solution (Figure 3-3f-g), and no additional precipitation occurred by further addition of 2 . Single crystals of the inclusion complex [2@1 $]^{2+}$ were formed on slow evaporation of the acetonitrile solvent at $318 \mathrm{~K}$ after several days.

\subsubsection{X-ray analysis}

a)

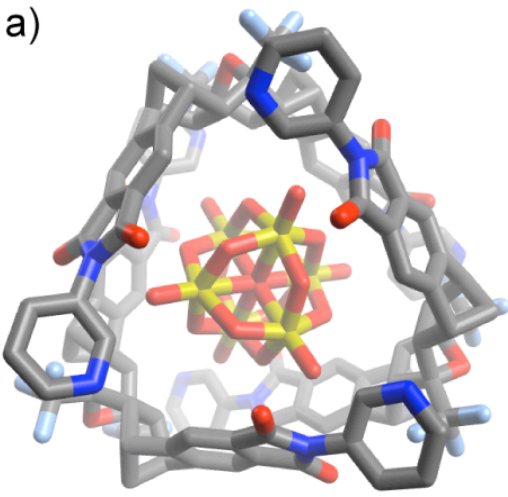

c)

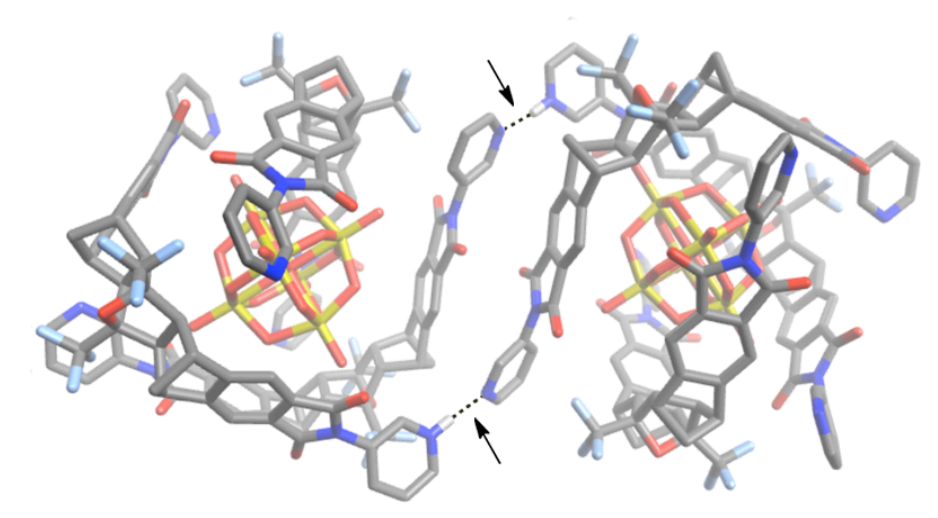

b)

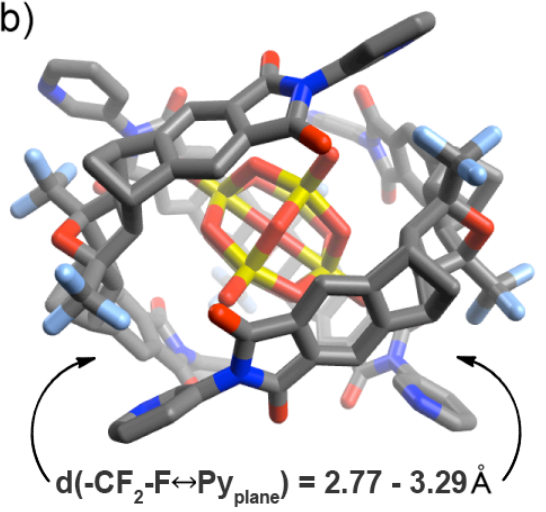

Figure 3-6 Structure of $\mathbf{3}$ in the crystal: (a) top and (b) side view highlighting two of the six close $\mathrm{CF}_{3}$-pyridine contacts. (c) Two neighbouring aggregates of $\mathbf{3}$ interact via $\mathrm{N}_{\mathrm{Py}}-\mathrm{H} \cdots \mathrm{N}_{\mathrm{Py}}$ hydrogen bonds (indicated by arrows). Other hydrogen atoms and solvent molecules have been omitted for clarity. C, gray; N, dark blue; O, red; F, light blue; Mo, orange. Copyright (C) 2012, American Chemical Society.

The crystallographic analysis of the species did not yield the anticipated molecular structure of [2@1][2], but instead revealed an unexpected result. All attempts to crystallize this compound resulted in the breakdown of the cage $\mathbf{1}$ and the loss of 
palladium cations. The compound was transformed to a new $\left\{2 @\right.$ (ligand) $\left.{ }_{3}+2 \mathrm{H}^{+}\right\}$assembly 3 (Figure 3-2), in which the hexamolybdate anion was surrounded by three ligands in a cyclic, chiral fashion (Figure 3-6), with an approximate $D_{3}$ symmetry. Among the six nitrogen atoms of the three ligands, two were found to be protonated. The residual water present, and subsequent formation of $\mathrm{Pd}(\mathrm{OH})_{2}$ was considered as a possible cause for the protonation of the ligands. The moieties are stabilized by intermolecular hydrogen bonds (Figure 3-6), which are formed between the protonated pyridine and the non-protonated pyridine of the neighbouring ligand strand $\left(\mathrm{N}_{\mathrm{Py}}-\mathrm{H} \cdots \mathrm{N}_{\mathrm{Py}}\right)$, as well as between the other protonated pyridine and an acetonitrile molecule $\left(\mathrm{N}_{\mathrm{Py}}-\mathrm{H} \cdots \mathrm{N}\right.$ ) (Figure 3-7). The other four nitrogen atoms of the remaining pyridines did not act as hydrogen bond donors, either because of the lack of possible acceptors in an appropriate distance or because the nitrogen atoms acted as acceptors themselves. Surprisingly, no $\mathrm{N}_{\mathrm{Py}}-\mathrm{H} \cdots \mathrm{O}$ hydrogen bonds were observed between the protonated pyridine and the hexamolybdate cluster.

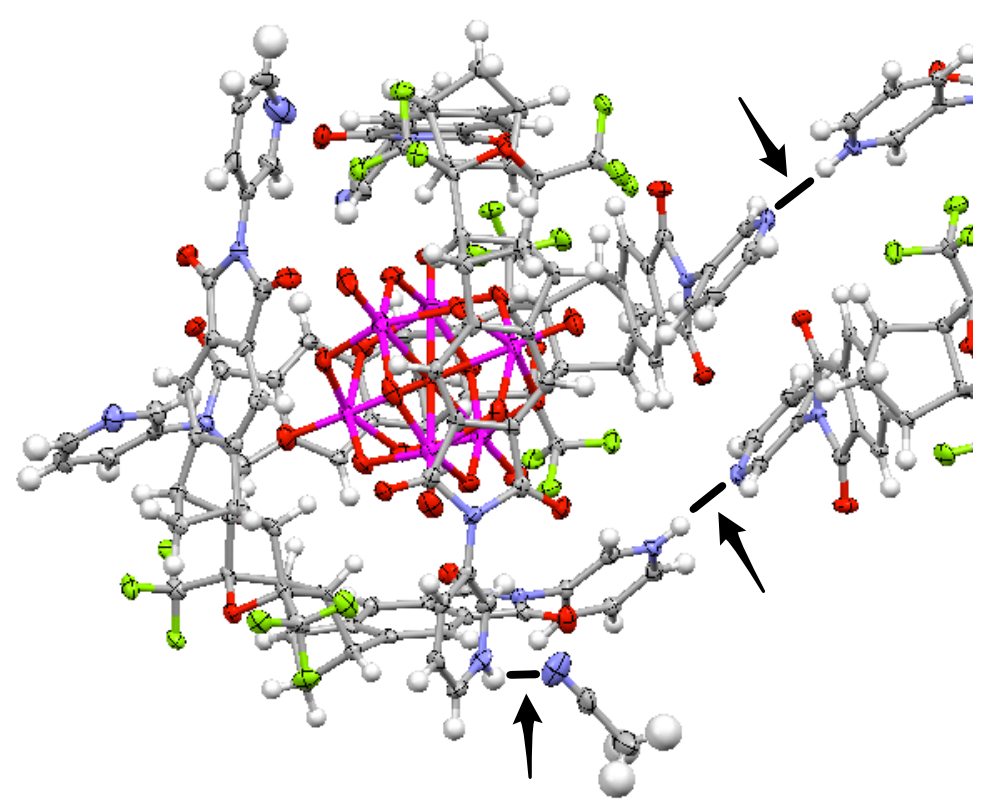

Figure 3-7 Ellipsoid drawing of the X-ray structure of 3. The hydrogen bonds are shown. Solvent molecules except for one MeCN are omitted for clarity.

Besides the existence of the intermolecular hydrogen bonds, several close $\mathrm{C}-\mathrm{H} \cdots \mathrm{O}$ contacts were observed between the hydrogen atoms of the 7-oxabicyclo[2.2.1]heptane moieties of the ligands and the oxygen atoms of the hexamolybdate cluster. One third of the aggregate is presented in Figure 3-8, which contains only the central part of one ligand with a protonated pyridine and unprotonated pyridine parts from the other two ligands. 


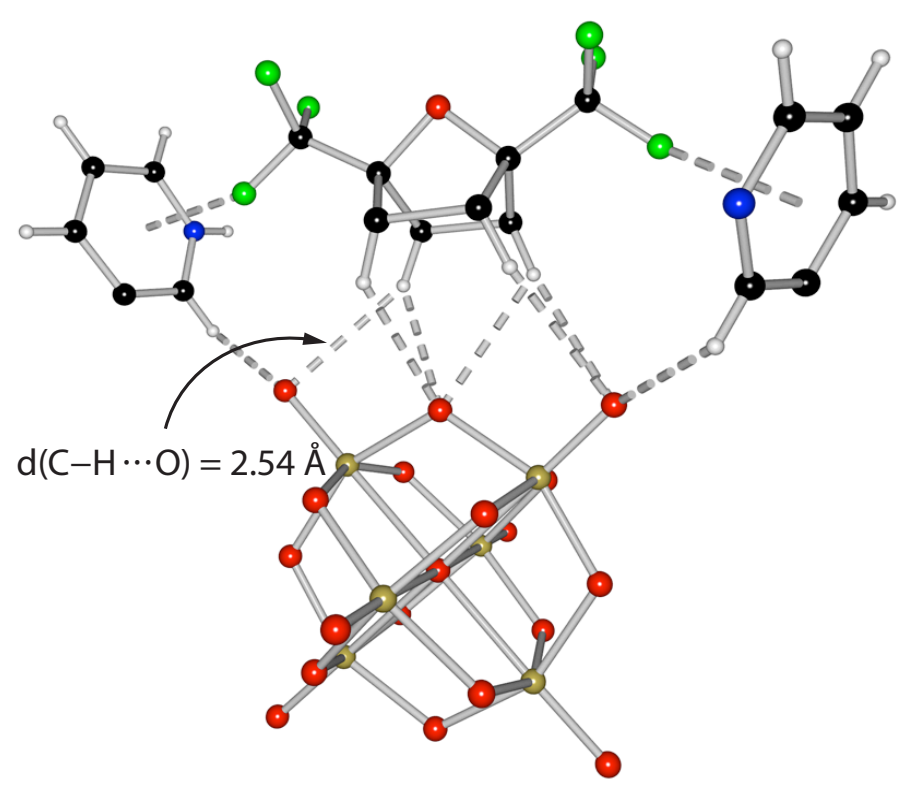

Figure 3-8 Ball-and-stick drawing of the observed $\mathrm{C}-\mathrm{H} \cdots \mathrm{O}$ and the $\mathrm{C}-\mathrm{F} \cdots$ Plane $\mathrm{Py}_{\mathrm{Py}}$ contacts (dashed lines).

It should be noted that the supramolecular aggregate $\mathbf{3}$ was probably not only stabilized by the hydrogen bonds as aforementioned, but also stabilized by the relative close $\mathrm{CF}_{3}$-pyridine plane contacts among all three ligands. All the interactions between the wrapping ligands with the hexamolybdate cluster are displayed in Figure 3-9. The $D_{3}$ symmetry can be recognized easily though this orientation, and all the $\mathrm{C}-\mathrm{H} \cdots \mathrm{O}$ and the C-F...Plane ${ }_{\text {Py }}$ contacts can be clearly seen. The shortest $\mathrm{H} \cdots \mathrm{O}$ distance is $2.54 \AA \AA^{,[43,44]}$ and the distances from the fluorine atom to its adjacent pyridine plane ranges from 2.77 to $3.29 \AA ̊$. The closest C-F...Plane $e_{\text {Py }}$ distance is $2.95 \AA$. 


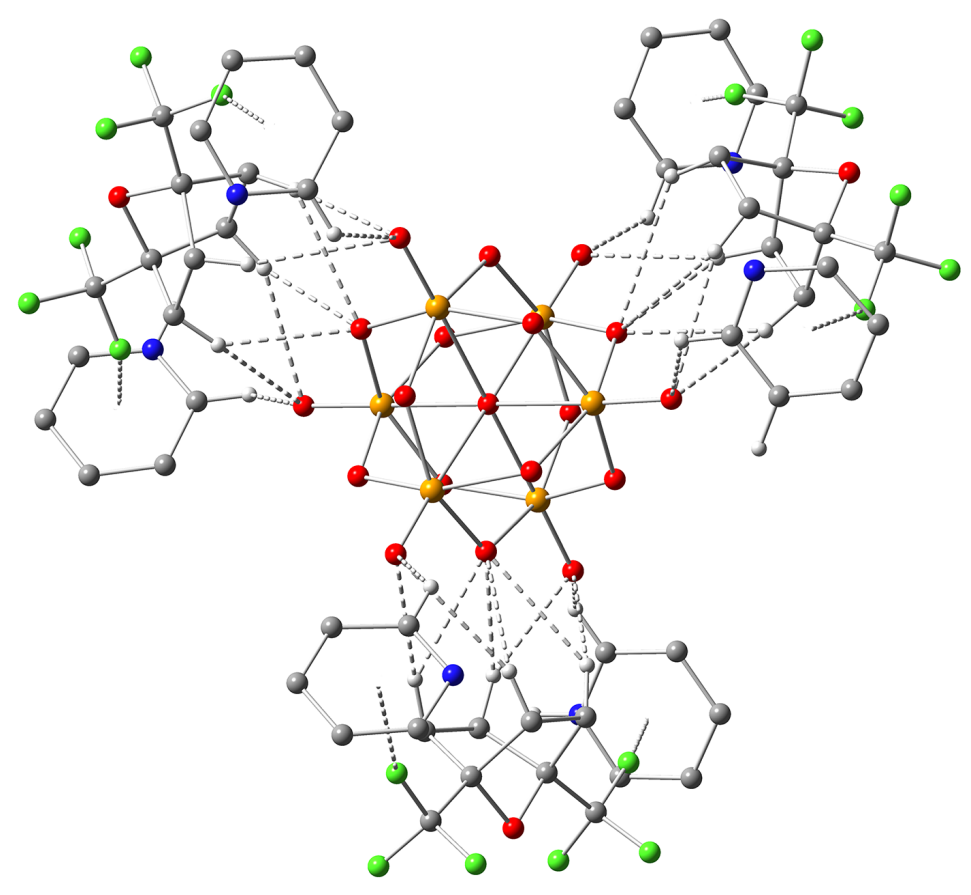

Figure 3-9 Graphical illustration of the hexamolybdate cluster and interacting parts of the surrounding ligands. The $\mathrm{C}-\mathrm{H} \cdots \mathrm{O}$ and the $\mathrm{C}-\mathrm{F} \cdots \mathrm{Plane}_{\mathrm{Py}}$ contacts are indicated (dashed lines), while for clarity, all ligand atoms except those of the pyridyl and the 7oxabicyclo[2.2.1] heptane moieties are omitted.

\subsubsection{Quantum mechanical calculation of the C-F...Plane $e_{\mathrm{Py}}$ interaction}

In order to calculate the $\mathrm{CF}_{3}-\pi$ interaction strength of among the six $\mathrm{CF}_{3}$-goups at the top of

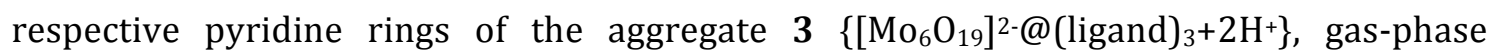
quantum mechanical $(\mathrm{QM})$ calculations were performed on the MP2/6-311++G(d,p) level of theory.

1,1,1-Trifluoroethane, pyridine, and $N$-protonated pyridinium were chosen and optimized by DFT calculation on the B3LYP/6-31G(d) level. The $\mathrm{CF}_{3}$ group was located above the pyridine or pyridinium ring plane, with one fluorine atom pointing towards the ring plane. The $\mathrm{C}_{\mathrm{CF} 3}-\mathrm{F}$ bond is perpendicular to the ring plane (parallel to the normal vector of the plane), and the $\mathrm{C}_{\mathrm{CH} 3}-\mathrm{C}_{\mathrm{CF} 3}$ bond forming a $180^{\circ}$ dihedral angle with the $\mathrm{N}-\mathrm{C}_{4}$ axis of the pyridine of the pyridinium ring. A rigid, 3-dimensional potential energy surface (PSE) scan was performed on the B3LYP/6-31G(d) level of theory by varying the in-plane coordinates $(x, y)$ and the F-ring distance $(z)$ in accordance with Figure 3-10. The energy minimum of the unprotonated pyridine deviated slightly from the center of the ring (position 13 in Figure 3-10a), with the coordinates $x=2.00, y=2.00, z=2.90$. The distance between the nearest fluorine atom of the $\mathrm{CF}_{3} \mathrm{CH}_{3}$ and the ring plane $(2.90 \AA)$ is well consistent with the distances found by X-ray structure. 
a)

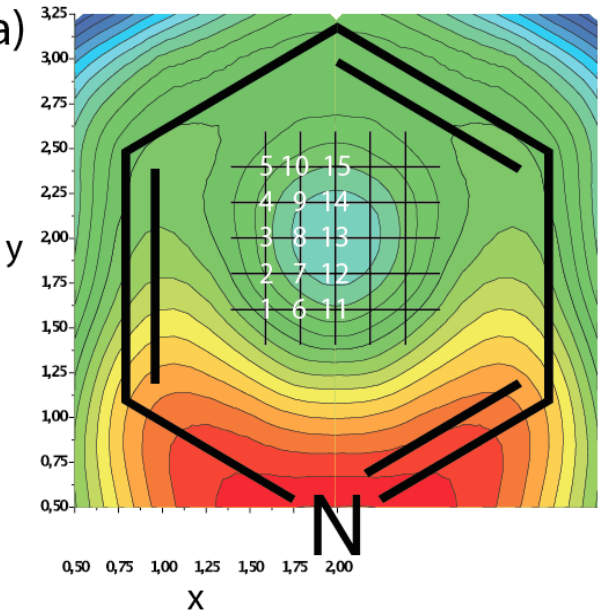

b)

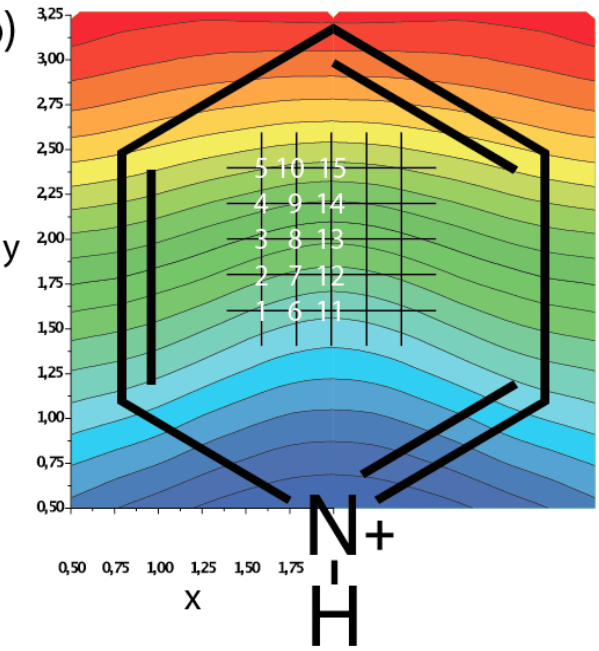

Figure 3-10 The PES scan result plots reflecting the interaction of 1,1,1-trifluoroethane respectively with a) pyridine and b) the N-protonated pyridinium cation. Slices at $z=3.00$ of the first PES scans at B3LYP/6-31G(d) level $(x=0.50-2.00$, incr. 0.25; $y=0.50-3.25$, incr. $0.25 ; z=2.00-7.00$, incr. 0.10 ; all distances in $\AA$ ). Red reflects high energy while blue for low energy. The grids marked with numbers represent the $x y$ positions that would be used for the second-round PES scan on a higher level of theory (see below).

A PES scan was performed on a higher level of theory (MP2/6-311++G(d,p)) to obtain the relative positions of the $\mathrm{CF}_{3} \mathrm{CH}_{3}$ with respect to both pridine and pyridinium planes. It presented a minimum on the potential energy surface at the distance of F-pyridine ring plane of $2.9 \AA$, providing an energetic stabilization of about $11.4 \mathrm{~kJ} \mathrm{~mol}^{-1}$ (Figure 3 -11a), and a minimum in a distance of $2.8 \AA$ for the protonated model about $20.4 \mathrm{~kJ} \mathrm{~mol}^{-1}$ (Figure 3-11b), although the enlarged view of it showed the position 11 was the energy minimum at first glance.

F-pyridine: $\mathrm{E}$ at position $13(x=2.00, y=2.00): \mathrm{E}(z=8.00)-\mathrm{E}(z=2.90)=\underline{11.4 \mathrm{~kJ} \mathrm{~mol}^{-1}}$ F-pyridinium: $\mathrm{E}$ at position $13(x=2.00, y=2.00): \mathrm{E}(z=8.00)-\mathrm{E}(z=2.80)=20.4 \mathrm{~kJ} \mathrm{~mol}^{-1}$ F-pyridinium: $\mathrm{E}$ at position $11(x=2.00, y=2.00): \mathrm{E}(z=8.00)-\mathrm{E}(z=2.80)=22.1 \mathrm{~kJ} \mathrm{~mol}^{-1}$ This result is consistent with the data reported previously including the structural analysis of X-ray and QM calculations of a variety of $\mathrm{C}-\mathrm{F} \cdots \pi$ contacts involving pyridine and other electron-deficient aromatic systems of this kind.[46,47] 
a)
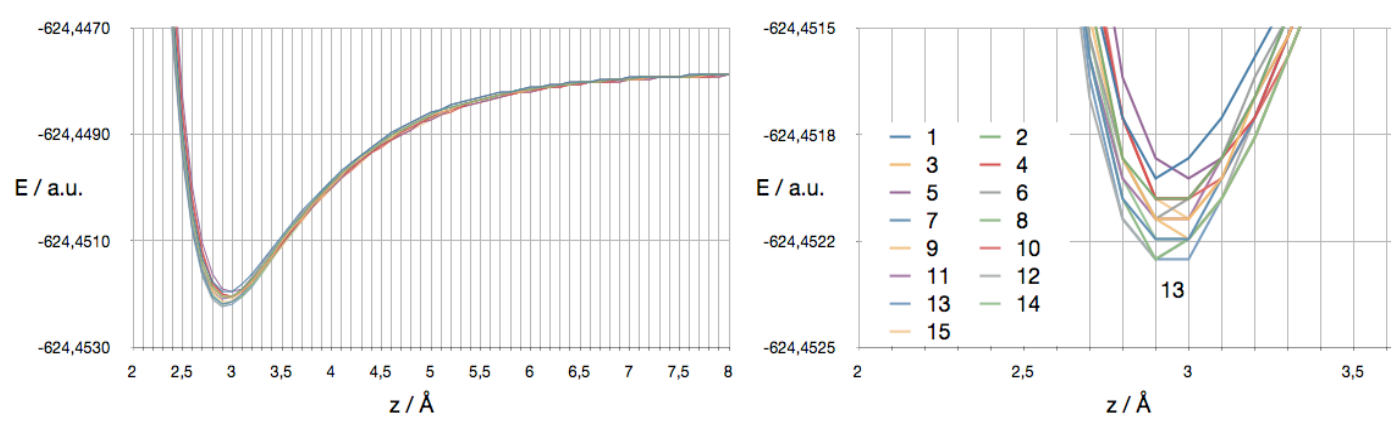

b)
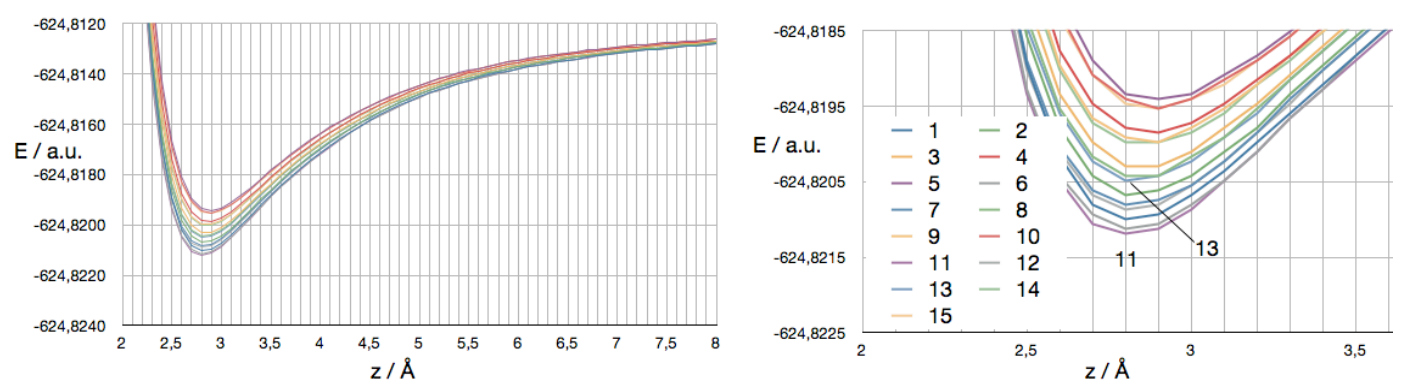

Figure 3-11 Second PES scan of a) pyridine- $\mathrm{CF}_{3} \mathrm{CH}_{3}$ and b) pyridinium- $\mathrm{CF}_{3} \mathrm{CH}_{3}$ at $\mathrm{MP} 2 / 6$ $311++G(d, p)$ level $(x=1.60-2.00$, incr. 0.20; $y=1.60-2.40$, incr. 0.20; $z=2.00-8.00$, incr. 0.10). Left: full view of all computed potential curves superimposed; right: detail around the calculated minima. The numbers in the detail on the right side refer to the xy positions shown in Figure 3-10.

From the gas-phase calculation on the simplified model system above, the enthalpic contribution of the six noncovalent F...pyridine/pyridinium interactions holding together the aggregate $\left.3\left\{\left[\mathrm{Mo}_{6} \mathrm{O}_{19}\right]^{2-@(l i g a n d}\right)_{3}+2 \mathrm{H}^{+}\right\}$could be roughly calculated: $4 \times \mathrm{F}$-pyridine + $2 \times \mathrm{F}-$ pyridinium $=4 \times 11.4 \mathrm{~kJ} \mathrm{~mol}^{-1}+2 \times 20.4 \mathrm{~kJ} \mathrm{~mol}^{-1}=86.4 \mathrm{~kJ} \mathrm{~mol}^{-1}$

Therefore, it is expect that, upon recrystallization, the structural conversion from [2@1][2] into 3 can be attributed to the cyclic arrangement of six of these noncovalent interactions and the multiple $\mathrm{C}-\mathrm{H} \cdots \mathrm{O}$ hydrogen bonds.

\subsection{Conclusion}

This chapter discussed the encapsulation of a hexamolybdate dianion, a Lindqvist type of POM, inside a discrete coordination host compound, and the host's structural conversion upon adding excess amount of guest molecules. The 1:1 host-guest compound [2@1] is 
anticipated to form microcrystalline [2@1][2] during addition of more than one equivalent of guest $\mathbf{2}$. This then undergoes a structureal conversion to form the aggregate

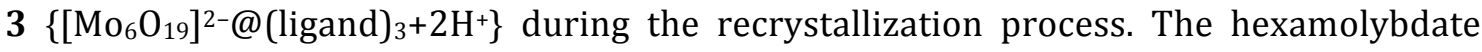
anion was wrapped by three ligands in the absence of any $\mathrm{Pd}^{\mathrm{II}}$ anions in a chiral, cyclic arrangement. This formation can be explained by intermolecular $\mathrm{C}-\mathrm{H} \cdots \mathrm{O}$ and C-F...Pyridine/pyridinium interactions. Moreover, the encapsulation of transition metal polyoxometalates in similar systems may help to stabilize unusual cluster compositions and structures based on templating and shielding effects imposed by the surrounding cage. This noncovalent approach of wrapping an organic shell around an inorganic cluster compound may lead to the generation of hybrid materials with new properties and functions.

\subsection{Experimental Section}

\subsubsection{Synthesis of [2@1] and [2@1][2]}

The bidentate pyridyl ligand $\left(\mathrm{C}_{40} \mathrm{H}_{24} \mathrm{~F}_{6} \mathrm{~N}_{4} \mathrm{O}_{5}, \mathbf{L}\right)$, cage $\mathbf{1}^{[38]}$ and the dianionic hexamolybdate salt $\left({ }^{n} \mathrm{Bu}_{4} \mathrm{~N}\right)_{2} \mathrm{Mo}_{6} \mathrm{O}_{19} 2^{[48]}$ were synthesized following the literature procedures.

Inclusion complex [2@1] $\left(\mathrm{BF}_{4}\right)_{2}$ and precipitate [2@1][2]:

$\left({ }^{n} \mathrm{Bu}_{4} \mathrm{~N}\right)_{2} \mathrm{Mo}_{6} \mathrm{O}_{19} 2$ (11.76 mg, $8.62 \mu \mathrm{mol}$ dissolved in $400 \mu \mathrm{L} \mathrm{CD}{ }_{3} \mathrm{CN}, 1$ equiv.) was added into a solution of cage 1 in $\mathrm{CD}_{3} \mathrm{CN}(1.0 \mathrm{~mL}, 4.31 \mathrm{mM})$. The quantitative formation of a soluble host-guest complex [2@1] $\left(\mathrm{BF}_{4}\right)_{2}$ occurred (confirmed by NMR spectroscopy and ESI mass spectrometry). Upon the addition of a second equivalent of 2 led to the gradual disappearing of the cage signals, while a thick, pale yellow precipitate [2@1][2] was formed. The sample was further stirred for $30 \mathrm{~min}$ to ensure completion of the reaction. The precipitate was washed several times with small portions of acetonitrile, and dried overnight in vacuo at room temperature (15.24 mg, 70\%).

${ }^{1} \mathbf{H}$ NMR of [2@1] $\left(\mathrm{BF}_{4}\right)_{2}\left(500 \mathrm{MHz}, 298 \mathrm{~K}, \mathrm{CD}_{3} \mathrm{CN}\right): \delta 1.60\left(8 \mathrm{H}, \mathrm{d},{ }^{2} J=10.4 \mathrm{~Hz}, \mathrm{H}_{\mathrm{a}}\right), 2.34$ $\left(16 \mathrm{H}, \mathrm{s}, \mathrm{H}_{\mathrm{b}}\right), 2.54\left(8 \mathrm{H}, \mathrm{d},{ }^{2} J=9.9 \mathrm{~Hz}, \mathrm{H}_{\mathrm{c}}\right), 3.84\left(16 \mathrm{H}, \mathrm{s}, \mathrm{H}_{\mathrm{d}}\right), 7.66\left(16 \mathrm{H}, \mathrm{s}, \mathrm{H}_{\mathrm{f}}\right), 7.71\left(8 \mathrm{H}, \mathrm{dd},{ }^{3} J=\right.$ $\left.8.3 \mathrm{~Hz},{ }^{4}=5.8 \mathrm{~Hz}, \mathrm{H}_{\mathrm{e}}\right), 8.25-8.32\left(16 \mathrm{H}, \mathrm{m}, \mathrm{H}_{\mathrm{g},} \mathrm{H}_{\mathrm{h}}\right), 8.41\left(8 \mathrm{H}, \mathrm{d},{ }^{4} J=1.8 \mathrm{~Hz}, \mathrm{H}_{\mathrm{i}}\right)$.

ESI-MS: $m / z\left([\mathbf{2} @ \mathbf{1}]^{2+}\right)$ calcd. $=2055.41$, found $=2055.37$.

\section{Elemental Analysis (\%):}

Calc. for $\mathrm{C}_{160} \mathrm{H}_{96} \mathrm{~F}_{24} \mathrm{Mo}_{12} \mathrm{~N}_{16} \mathrm{O}_{58} \mathrm{Pd}_{2}\left(\mathrm{H}_{2} \mathrm{O}\right)_{10}: \quad$ C 37.42, H 2.20, N 4.36;

Found:

C 37.17, H 2.38, N 4.38. 


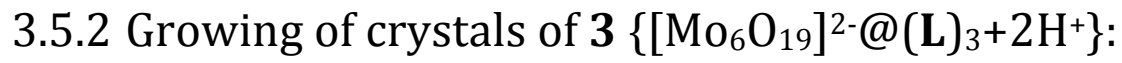

A suspension of the pale yellow precipitate of [2@1][2] in acetonitrile was allowed to evaporate slowly at $50{ }^{\circ} \mathrm{C}$. Within several days, light yellow crystals of 3 suitable for single crystal structure determination were formed and subjected to X-ray analysis as described in 2.5.4.

\subsubsection{Separate Pd-free synthesis of $\left.\left\{\left[\mathrm{Mo}_{6} \mathrm{O}_{19}\right]^{2-@(L)}\right)_{3}+2 \mathrm{H}^{+}\right\} \times 1.5$ PTSA}

In order to obtain more information about the aggregate $\mathbf{3}$ in solution, a parallel experiment was prepared by using $p$-toluenesulfonic acid monohydrate (PTSA) as protonation source. A solution of $\left({ }^{n} \mathrm{Bu}_{4} \mathrm{~N}\right)_{2} \mathrm{Mo}_{6} \mathrm{O}_{19}$ (9.04 mg, $6.63 \mu \mathrm{mol}, 1$ equiv.) in MeCN $(300 \mu \mathrm{L})$ was added dropwise to a mixture of the ligand (15 mg, $19.88 \mu \mathrm{mol}, 3$ equiv.) and PTSA (2.52 mg, $13.25 \mu \mathrm{mol}, 2$ equiv.) in $\mathrm{MeCN}(500 \mu \mathrm{L})$. The resulting precipitate was collected, washed with $\mathrm{MeCN}$, and dried in vacuo to give 3 co-precipitated with 1.5 equiv. of PTSA as an off-white powder (16.67 mg, 74\%).

${ }^{1} \mathrm{H}$ NMR spectroscopy and elemental analysis confirmed the co-precipitation of 1.5 equiv. of PTSA. The sample was obtained by complete dissolution of the precipitate in DMSO- $d_{6}$

1H NMR (300 MHz, $\left.298 \mathrm{~K}, \mathrm{DMSO}-d_{6}\right) \delta 1.59\left(6 \mathrm{H}, \mathrm{d},{ }^{2} J=10.2 \mathrm{~Hz}, \mathrm{H}_{\mathrm{a}}\right), 2.29\left(12 \mathrm{H}, \mathrm{s}, \mathrm{H}_{\mathrm{b}}\right), 2.46$ $\left(6 \mathrm{H}, \mathrm{d},{ }^{2} J=10.0 \mathrm{~Hz}, \mathrm{H}_{\mathrm{c}}\right), 3.89\left(12 \mathrm{H}, \mathrm{s}, \mathrm{H}_{\mathrm{d}}\right), 7.12\left(3 \mathrm{H}, \mathrm{d},{ }^{3} \mathrm{~J}=8.4 \mathrm{~Hz}, \quad \mathrm{H}_{\text {arom, TsOH }}\right), 7.47\left(3 \mathrm{H}, \mathrm{d},{ }^{3} \mathrm{~J}\right.$ $\left.=8.4 \mathrm{~Hz}, \mathrm{H}_{\text {arom, } \mathrm{TsOH}}\right), 7.57\left(6 \mathrm{H}, \mathrm{dd},{ }^{3} J=7.8,4 J=4.2 \mathrm{~Hz}, \mathrm{H}_{\mathrm{e}}\right), 7.87\left(6 \mathrm{H}, \mathrm{m}, \mathrm{H}_{\mathrm{g}}\right), 7.98(12 \mathrm{H}, \mathrm{s}$, $\left.\mathrm{H}_{\mathrm{f}}\right), 8.60\left(6 \mathrm{H}, \mathrm{dd}, 3 J=4.8 \mathrm{~Hz}, 4 J=1.7 \mathrm{~Hz}, \mathrm{H}_{\mathrm{h}}\right), 8.64\left(6 \mathrm{H}, \mathrm{d},{ }^{4} J=1.8 \mathrm{~Hz}, \mathrm{H}_{\mathrm{i}}\right)$.

\section{Elemental Analysis (\%):}

Calc. for $\mathrm{C}_{130.5} \mathrm{H}_{86} \mathrm{~F}_{18} \mathrm{Mo}_{6} \mathrm{~N}_{12} \mathrm{O}_{38.5} \mathrm{~S}_{1.5}: \quad \mathrm{C} 46.05, \mathrm{H} 2.55, \mathrm{~N}$ 4.94;

Found:

C 46.37, H 2.77, N 5.05 .

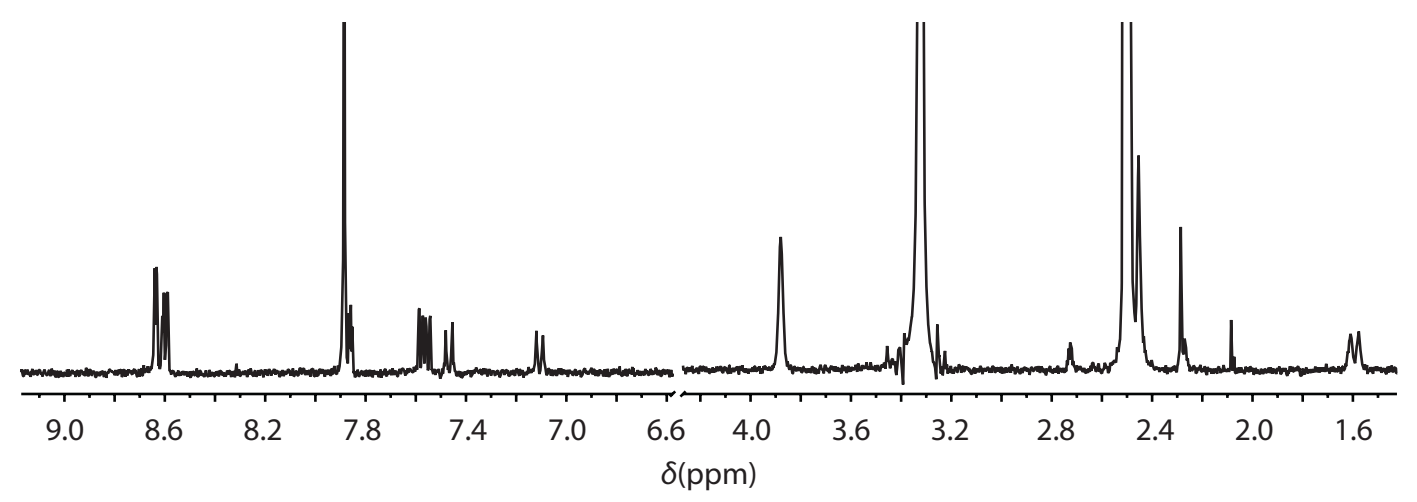

Figure 3-12 ${ }^{1} \mathrm{H}$ NMR $\left(300 \mathrm{MHz}\right.$, DMSO- $\left.d_{6}\right)$ spectrum of compound $\left\{\left[\mathrm{Mo}_{6} \mathrm{O}_{19}\right]^{2-}\right.$ $\left.@(\mathbf{L})_{3}+2 \mathrm{H}^{+}\right\} \times 1.5$ PTSA. 


\subsubsection{X-ray structure analysis of 3}

The X-ray structure was measured, solved and refined by Dr. Jakob Hey.

Table 3-1 Crystal data and structure refinement

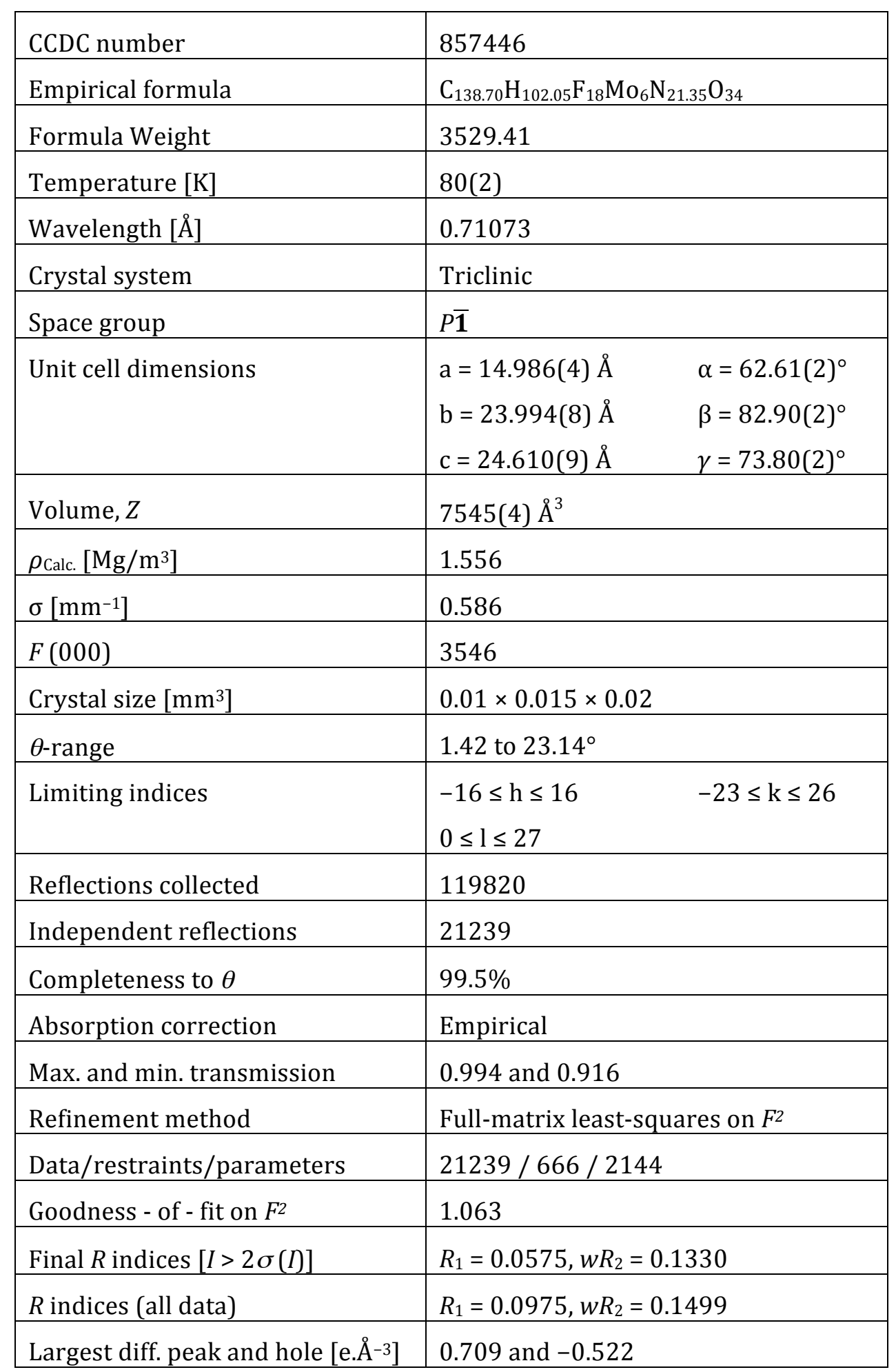


A crystal of a size of $0.01 \times 0.015 \times 0.02 \mathrm{~mm}^{3}$ was selected from a batch of crystals using the X-TEMP 2 device.[49,50] The crystal was mounted on top of glass fibre using inert perfluorinated polyether oil and placed in the cold gas stream of a Bruker Kryoflex 2 lowtemperature device with a cold stream at $80(2) \mathrm{K}$ on a Bruker three circle diffractometer. The diffractometer was equipped with a SMART APEX 2 CCD detector and a rotating anode source (Mo-K $\alpha ; \lambda=0.71073 \AA$ ).

The crystal used for data collection was very small. It could be seen that the crystal in fact was a split crystal, thus consisting of two domains with similar orientation. However, the crystal quality appeared to be above that of the remaining crystals in the batch and the split crystal was thus used for data collection.

Data was collected in omega-scan mode at different detector $2 \theta$ angles of $10^{\circ}$ and $20^{\circ}$ and several different $\varphi$ angles varying from scan to scan. The scan width was set to $0.3^{\circ}$.

Data integration was completed using SAINT 7.68A software.[51] The integration was done using both domains of the crystal. Data scaling, absorption correction and merging of the data were done with TWINABS 2008/4.[52] Two HKL files were written containing data in HKLF4 and HKLF5 format, respectively. The latter contained reflections from the stronger of the two domains as well as composite reflections.

The space group was determined using XPREP on the HKLF4 data. The structure was solved by direct methods using SHELXS-97 on the HKLF4 data. Refinement by full-matrix least-squares procedures was done with SHELXL-97 using the HKLF5 data.[53] The two observed pyridinium $\mathrm{N}-\underline{\mathrm{H}}$ hydrogen positions were picked from the Fourier difference map. The remaining four pyridyl nitrogen atoms could not be identified as possible hydrogen bond donors, either because of the lack of possible acceptors in any meaningful distance or because the nitrogen atoms acted as acceptors themselves.

\subsection{References}

[1] D.-L. Long, E. Burkholder, L. Cronin, Chem. Soc. Rev. 2006, 36, 105.

[2] J. T. Rhule, C. L. Hill, D. A. Judd, Chem. Rev. 1998, 98, 327.

[3] C. L. Hill (Ed.), J. Mol. Catal. A: Chem. 2007, 262, 1-242. (special issue on POM-based catalysis)

[4] E. Coronado, C. J. Gómez-García, Chem. Rev. 1998, 98, 273.

[5] W. G. Klemperer, C. G. Wall, Chem. Rev. 1998, 98, 297.

[6] M. T. Pope, A. Müller, Angew. Chem. Int. Ed. 1991, 30, 34.

[7] A. Proust, R. Thouvenot, P. Gouzerh, Chem. Commun. 2008, 1837.

[8] D.-L. Long, R. Tsunashima, L. Cronin, Angew. Chem. Int. Ed. 2010, 49, 1736. 
[9] A. Müller, E. Krickemeyer, J. Meyer, H. Bögge, F. Peters, W. Plass, E. Diemann, S. Dillinger, F. Nonnenbruch, M. Randerath, C. Menke, Angew. Chem. Int. Ed. 1995, 34, 2122.

[10] A. Müller, E. Krickemeyer, H. Bögge, M. Schmidtmann, F. Peters, Angew. Chem. Int. Ed. 1998, 37, 3360.

[11] A. Müller, E. Beckmann, H. Bögge, M. Schmidtmann, A. Dress, Angew. Chem. Int. Ed. 2002, 41, 1162.

[12] Z. Peng, Angew. Chem. Int. Ed. 2004, 43, 930.

[13] H. Kang, J. Zubieta, Chem. Commun. 1988, 1192.

[14] H. Kwen, V. C. Young, E. A. Maatta, Angew. Chem. Int. Ed. 1999, 38, 1145.

[15] Y. Wei, B. Xu, C. L. Barnes, Z. Peng, J. Am. Chem. Soc. 2001, 123, 4083.

[16] B. Xu, Z. Peng, Y. Wei, D. R. Powell, Chem. Commun. 2003, 2562.

[17] S. Bareyt, S. Piligkos, B. Hasenknopf, P. Gouzerh, E. Lacôte, S. Thorimbert, M. Malacria, J. Am. Chem. Soc. 2005, 127, 6788.

[18] S.-T. Zheng,; J. Zhang, X.-X. Li, W.-H. Fang, G.-Y. Yang, J. Am. Chem. Soc. 2010, 132, 15102.

[19] A. M. Todea, A. Merca, H. Bögge, T. Glaser, J. M. Pigga, M. L. K. Langston, T. Liu, R. Prozorov, M. Luban, C. Schröder, W. H. Casey, A. Müller, Angew. Chem. Int. Ed. 2010, 49, 514.

[20] C. Ritchie, E. Burkholder, P. Kögerler, L. Cronin, Dalton Trans. 2006, 1712.

[21] M. Grabau, J. Forster, K. Heussner, C. Streb, Eur. J. Inorg. Chem. 2011, 1719.

[22] H. Zeng, G. R. Newkome, C. L. Hill, Angew. Chem. Int. Ed. 2000, 39, 1771.

[23] C. Ritchie, C. Streb, J. Thiel, S. G. Mitchell, H. N. Miras, D.-L. Long, T. Boyd, R. D. Peacock, T. McGlone, L. Cronin, Angew. Chem. Int. Ed. 2008, 47, 6881.

[24] T. McGlone, C. Streb, M. B. Fite, J. Yan, D. Gabb, D.-L. Long, L. Cronin, Cryst. Growth Des. 2011, 11, 2471.

[25] Y. Sakai, S. Ohta, Y. Shintoyo, S. Yoshida, Y. Taguchi, Y. Matsuki, S. Matsunaga, K. Nomiya, Inorg. Chem. 2011, 50, 6575.

[26] C. Streb, D.-L. Long, L. Cronin, Chem. Commun. 2007, 471.

[27] S. Favette, B. Hasenknopf, J. Vaissermann, P. Gouzerh, C. Roux, Chem. Commun. 2003, 2664.

[28] F. Odobel, M. Severac, Y. Pellegrin, E. Blart, C. Fosse, C. Cannizzo, C. R. Mayer, K. J. Elliott, A. Harriman, Chem. Eur. J. 2009, 15, 3130.

[29] C.-F. Lee, D. A. Leigh, R. G. Pritchard, D. Schultz, S. J. Teat, G. A. Timco, R. E. P. Winpenny, Nature 2009, 458, 314.

[30] J. Zhang, J. Hao, Y. Wei, F. Xiao, P. Yin, L. Wang, J. Am. Chem. Soc. 2010, 132, 14.

[31] X. Fang, P. Kögerler, L. Isaacs, S. Uchida, N. Mizuno, J. Am. Chem. Soc. 2009, 131, 432.

[32] T. Chatterjee, M. Sarma, S. K. Das, Cryst. Growth Des. 2010, 10, 3149.

[33] X. Kuang, X.-Y. Wu, J. Zhanga, C.-Z. Lu, Chem. Commun. 2011, 4150.

[34] J. M. Knaust, C. Inman, S. W. Keller, Chem. Commun. 2004, 492.

[35] X. Kuang, X.-Y. Wu, R. Yu, J. P. Donahue, J. Huang, C.-Z. Lu, Nat. Chem. 2010, 2, 461.

[36] Y. Liu, C. Hu, A. Comotti, M. D. Ward, Science 2011, 333, 436. 
[37] K. Uehara, T. Oishi, T. Hirose, N. Mizuno, Inorg. Chem. 2013, 52, 11200.

[38] G. H. Clever, S. Tashiro, M. Shionoya, Angew. Chem. Int. Ed. 2009, 48, 7010.

[39] G. H. Clever, W. Kawamura, M. Shionoya, Inorg. Chem. 2011, 50, 4689.

[40] G. H. Clever, S. Tashiro, M. Shionoya, J. Am. Chem. Soc. 2010, 132, 9973.

[41] G. H. Clever, M. Shionoya, Chem. Eur. J. 2010, 16, 11792.

[42] G. H. Clever; W. Kawamura, S. Tashiro, M. Shiro, M. Shionoya, Angew. Chem. Int. Ed. 2012, 51, 2606.

[43] G. R. Desiraju, T. Steiner, The Weak Hydrogen Bond in Structural Chemistry and Biology; Oxford University Press: Oxford, U.K., 2001.

[44] G. R. Desiraju, Chem. Commun. 2005, 2995.

[45] Gaussian 09, M. J. Frisch, G. W. Trucks, H. B. Schlegel, G. E. Scuseria, M. A. Robb, J. R. Cheeseman, G. Scalmani, V. Barone, B. Mennucci, G. A. Petersson, H. Nakatsuji, M. Caricato, X. Li, H. P. Hratchian, A. F. Izmaylov, J. Bloino, G. Zheng, J. L. Sonnenberg, M. Hada, M. Ehara, K. Toyota, R. Fukuda, J. Hasegawa, M. Ishida, T. Nakajima, Y. Honda, O. Kitao, H. Nakai, T. Vreven, J. A. Montgomery, Jr., J. E. Peralta, F. Ogliaro, M. Bearpark, J. J. Heyd, E. Brothers, K. N. Kudin, V. N. Staroverov, R. Kobayashi, J. Normand, K. Raghavachari, A. Rendell, J. C. Burant, S. S. Iyengar, J. Tomasi, M. Cossi, N. Rega, J. M. Millam, M. Klene, J. E. Knox, J. B. Cross, V. Bakken, C. Adamo, J. Jaramillo, R. Gomperts, R. E. Stratmann, O. Yazyev, A. J. Austin, R. Cammi, C. Pomelli, J. W. Ochterski, R. L. Martin, K. Morokuma, V. G. Zakrzewski, G. A. Voth, P. Salvador, J. J. Dannenberg, S. Dapprich, A. D. Daniels, O. Farkas, J. B. Foresman, J. V. Ortiz, J. Cioslowski and D. J. Fox, Gaussian, Inc., Wallingford CT, 2009.

[46] T. V. Rybalova, I. Y. Bagryanskaya, J. Struct. Chem. 2009, 50, 741.

[47] S. Kawahara, S. Tsuzuki, T. Uchimaru, J. Phys. Chem. A 2004, 108, 6744.

[48] N. H. Hur, W. G. Klemperer, R. C. Wang, Inorg. Synth. 1990, 27, 77.

[49] T. Kottke, D. Stalke, J. Appl. Crystallogr. 1993, 26, 615.

[50] D. Stalke, Chem. Soc. Rev. 1998, 27, 171.

[51] Bruker, SAINT v7.68A, Madison, 2009.

[52] G. M. Sheldrick, TWINABS 2008/4, Göttingen, 2008.

[53] G. M. Sheldrick, Acta Crystallogr., Sect. A. 2008, 64, 112-122.

[54] W. Kawamura, Master Thesis, 2011, The Shionoya Lab, The University of Tokyo. 


\section{RATIONAL DESIGN OF A FACE- CENTRED SQUARE-CUBOID COORDINATION CAGE ${ }^{3}$}

In this chapter, the formation of a highly symmetric cubic cage $\operatorname{Pd}_{6} \mathbf{L}_{12}$ and a face-centred square cuboid $\operatorname{Pd}_{6} \mathbf{L}^{2}{ }_{8}$ will be described. Both of the ligands employed were synthesized from a dibenzosuberone backbone, as this style of ligand has been successfully used in the formation of interpenetrated coordination cages within the Clever group. The symmetry reduction from a cubic cage $(a=b=c)$ to a face-centred square cuboid $(a=b \neq c)$ cage will be discussed, and the special topological coordination environments of the face-centred cuboid will be explained.

\subsection{Introduction}

Although great efforts on defining synthetic methodologies and assembly strategies have been made in order to achieve specific supramolecular structures with unusual topologies or important functionalities, the construction of supramolecular architectures has to follow a range of restrictions imposed by the rules of physics and chemistry.[1] Some common limitations must be overcome during the design of the supramolecular self-

\footnotetext{
3 The work presented in this chapter has been published: M. Han, R. Michel, G. H. Clever, Chem. Eur. J. 2014, 34, 10640.
} 
assemblies, such as steric and electronic effects, stereochemical interactions, backbone flexibility, and favoured conformations. Moreover, other influential factors should be considered as well, like selection of solvents, neighbouring molecules, size of the counteranions.

Using supramolecular self-assembly strategies, chemists have overcome the limitations imposed by multistep covalent synthesis, being able to form complex structures from simple building blocks.[2,3] In particular, the quantitative formation of discrete well-defined three-dimensional metal-organic compounds such as helicates, knots, links,[4-6] rotaxanes, ${ }^{[7,8]}$ and coordination cages ${ }^{[9-15]}$ has been developed immensely.

Discrete assemblies constructed out of only one type of metal ion and identical ligands, which correspond to Platonic and Archimedean solids, are often highly symmetric.[16] Designing and quantitatively synthesising self-assembled polyhedra can be challenging, especially when a polyhedron with unequal faces is desired. In order to develop welldefined nanoscopic cavities which functions in selective recognition, transportation and catalysis,[17] a rational process to reduce the symmetry of the cage seems to be necessary.

Heteronuclear self-assemblies are one possible means to achieve reduced symmetry cages, although little research has been done on this topic. Raymond and Wong reported a heteronuclear trigonal prism using $\mathrm{Ti}^{\mathrm{IV}}$ and $\mathrm{Pd}^{\mathrm{II}}$ with phosphanylcatechol ligands, ${ }^{[18]}$ and soon afterwards, a similar system containing the same metal centres but pyridyl-catechol ligands was introduced by Hiraoka et al..[19] Stang and co-workers presented a trigonal bipyramidal cage based on octahedral $\mathrm{Ga}^{\mathrm{III}}$ or $\mathrm{Al}^{\mathrm{III}}$ and square-planar $\mathrm{Pt}^{\mathrm{II}}{ }^{[20]}{ }^{\text {Yoshizawa }}$ et al. described a trigonal prism-shaped molecule constructed of methyl substituted bipyridine and unsubstituted bipyridine with cis-protected $\mathrm{Pd}^{\mathrm{II}}$ by a heteroleptic coordination.[21] Plenty of homonuclear, heteroleptic prisms have been reported.[22-28] A few tetragonal prisms using a heteroleptic arrangement of ligands with homonulearic metal ions have been reported.[29] Fujita and co-workers developed square tubes by linking the end-capped $\mathrm{Pd}^{\mathrm{II}}$ cations with tetrakis-pyridyl ligands.[30,31] Besides, Wang et al. reported a tetragonal prism via coordination-driven self-assembly from combination of tetraphenylethylene based tetradentate ligands, linear dipyridines and cis-capped $\mathrm{Pd}^{\mathrm{II}}$.[32] Even the coordination of zinc porphyrin based panels with ruthenium as connectors was used to build a cubic shaped cage quantitatively.[33]

The formation of these low symmetric self-assemblies, which is always, achieved using either more than one kind of metal connectors or heteroleptic coordination environment. However, a great number of homoleptic, highly symmetric cages is built on the $\operatorname{Pd}(\text { pyridine })_{4}$ motifs.[34] Lately, some self-assembled structures also based on Pd(pyridine $)_{4}$ motifs with rather complicated topologies were introduced. By careful 
ligand design, the self-assembled structures, such as a giant sphere-in-sphere molecule[35] and a stellated cuboctahedron,[36] were formed quantitatively without leading to misassembled side products. To best of our knowledge, a square-cuboid self-assembly, in which all metal centres are the same and surrounded by identical pyridyl ligands, has not been reported.

The precise design and synthesis of a cubic cage $\mathrm{Pd}_{6} \mathrm{~L}_{12}$ and an unusual face-centred square-cuboid coordination cage $\mathrm{M}_{6} \mathrm{~L}_{8}$, based on Pd(pyridine) ${ }_{4}$ motifs, will be discussed in this chapter (Figure 4-1). The formation of cubic cages based on face-centred squareplanar metal cations and almost right-angled carboxylate ligands[37,38] or bis-pyridyl ligands ${ }^{[39]}$ was reported previously (Figure 4-1a).[40-43]

a)

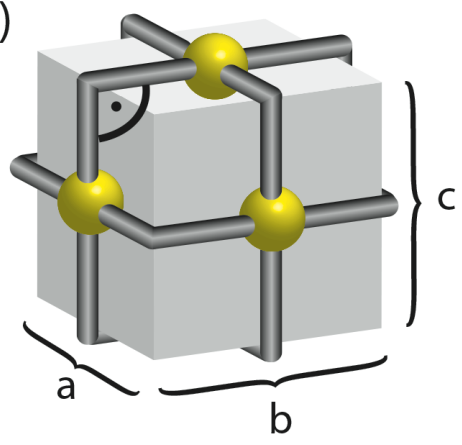

$\mathrm{a}=\mathrm{b}=\mathrm{c}$ b)

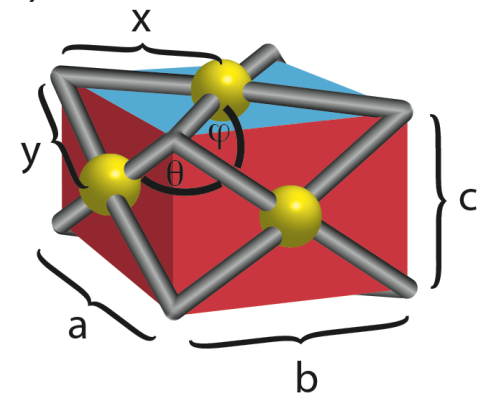

$a=b \neq c$

Figure 4-1 Schematic models of a) a cube based on right-angled bis-monodentate ligands and face-centred square-planar metal nodes (all side lengths are same and all faces are equal) and b) a square-cuboid cage composed of eight tripodal ligands, in which the side lengths of the top and bottom faces (blue) are equal and the lengths of the ligand arms are indicated with $x$ and $y$ and the angles between the arms with $\varphi$ and $\theta$. Copyright (C) 2014 WILEY-VCH Verlag GmbH \& Co.

\subsection{Derivation of cage geometry and mathematical modelling}

Starting from an $O_{h}$-symmetric cubic structure, whose side lengths are equal $(a=b=c)$, the symmetry can be lowered by altering only one side length and retaining the other two sides $(a=b \neq c)$ to achieve a square-cuboid cage with $D_{4 h}$ symmetry (Figure 4-1b).

It was found that the suitable shape and appropriate donor site orientation of a tripodal trispyridyl ligand could be used to construct a face-centred self-assembly as depicted in Figure 4-1b. Such tripodal ligands sit on all eight corners of the square-cuboid structure and coordinate with square-planar metal centres. The tripodal ligand has one central arm with length of $x$ and two equal long side arms with length of $y$, and the angle between the central arm and one side is $\varphi$, between the two side arms is $\theta$. In order to find the 
relationships between the side lengths $a$ and $c$ of the square-cuboid structure and the lengths and angles of the ligand building blocks, a derivation of the cage geometry was applied.

Each tripodal ligand can be considered by using one line segment $\overline{A O}$, which indicates the middle ligand arm, and two segments $\overline{A G}$ and $\overline{A F}$ for the two equal side arms. The angles $\angle O A F$ and $\angle O A G$ are defined between the central $\overline{A O}$ arm and each side arm $\overline{A G}$ and $\overline{A F}$. $\angle G A F$ presents the angle between the two side arms (Figure 4-2).

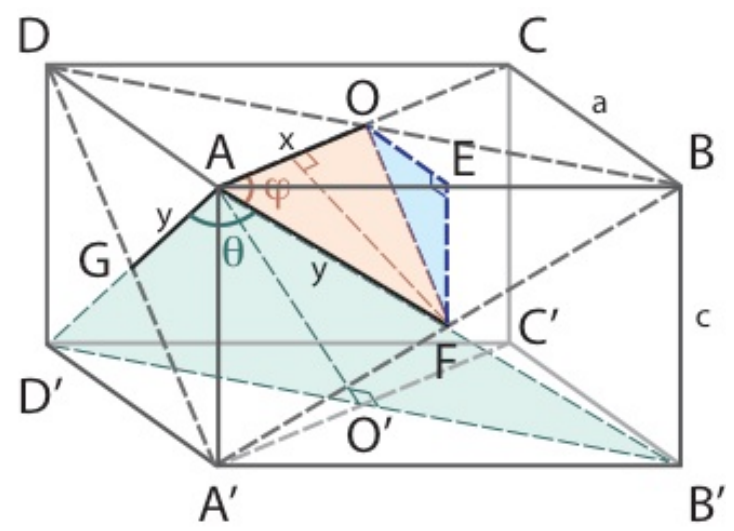

Figure 4-2 Geometry diagram of face-centred square-cuboid structure. Side lengths and angles are depicted.

Any tripodal ligand must match the following conditions, in order to form a face-centred $\operatorname{Pd}_{6} \mathbf{L}_{8}$ square-cuboid cage:

The bases of a square-cuboid are $A B C D$ and $A^{\prime} B^{\prime} C^{\prime} D^{\prime}$, with

$$
\begin{gathered}
\overline{A B}=\overline{B C}=\overline{C D}=\overline{D A}=a \\
\overline{A A^{\prime}}=\overline{B B^{\prime}}=\overline{C C^{\prime}}=\overline{D D^{\prime}}=c \\
\overline{O A}=x, \overline{A F}=\overline{A G}=y \\
\angle D^{\prime} A B^{\prime}=\theta, \quad \angle O A F=\angle O A G=\varphi
\end{gathered}
$$

$\mathrm{O}^{\prime}$ is the midpoint of $\overline{B^{\prime} D^{\prime}}$, draw $\mathrm{AD}^{\prime}, \mathrm{AB}^{\prime}, \mathrm{AO}^{\prime}, \overline{A O^{\prime}} \perp \overline{B^{\prime} D^{\prime}}$,

$$
\begin{gathered}
\overline{A D^{\prime}}=\overline{A B^{\prime}}=2 y \\
\left(\overline{B^{\prime} D^{\prime}}\right)^{2}=(2 x)^{2}=\left(\overline{D^{\prime} A^{\prime}}\right)^{2}+\left(\overline{A^{\prime} B^{\prime}}\right)^{2}=(a)^{2}+(a)^{2}=2 a^{2} \\
\overline{B^{\prime} D^{\prime}}=2 x=\sqrt{2} a \\
\sin \left(\frac{\theta}{2}\right)=\frac{\overline{B^{\prime} O^{\prime}}}{\overline{A^{\prime} B^{\prime}}}=\frac{x}{2 y}
\end{gathered}
$$




$$
\theta=2 \sin ^{-1}\left(\frac{x}{2 y}\right)
$$

Let $\mathrm{E}$ be the midpoint of $\mathrm{AB}$, then connect $O E, E F, F O$, and draw $\overline{O E} \perp \overline{E F}$,

$$
\begin{gathered}
\overline{O E}=\frac{a}{2}, \quad E F=\frac{c}{2} \\
(\overline{O F})^{2}=\left(\frac{a}{2}\right)^{2}+\left(\frac{c}{2}\right)^{2}=(\overline{A F})^{2}=y^{2}
\end{gathered}
$$

So the triangle $\triangle A F O$ is isosceles.

$$
\begin{gathered}
\cos \varphi=\frac{\overline{A O}}{2 \overline{A F}}=\frac{x}{2 y} \\
\varphi=\cos ^{-1}\left(\frac{x}{2 y}\right)
\end{gathered}
$$

The mathematical analysis reveals that there is a single combination of the ratio of the angles $\varphi / \theta$ and the ratio of the arm lengths $x / y$ for every possible face-centred squarecuboid cage structure.

$$
\frac{\varphi}{\theta}=\frac{\cos ^{-1}\left(\frac{1}{2} \cdot \frac{x}{y}\right)}{2 \sin ^{-1}\left(\frac{1}{2} \cdot \frac{x}{y}\right)}
$$

Then $\varphi / \theta$ is then plotted over $x / y$ based on this function (Figure 4-3a) while obeying the following boundary conditions:

1. The graph in the negative domain will not be considered.

2. Due to geometric considerations, the $\varphi / \theta$ and $x / y$ ratios must take values in the following ranges:

$$
\begin{gathered}
0.5<\frac{\varphi}{\theta}<\infty \\
0<\frac{x}{y}<\sqrt{2} \\
0<\frac{a}{c}<\infty
\end{gathered}
$$




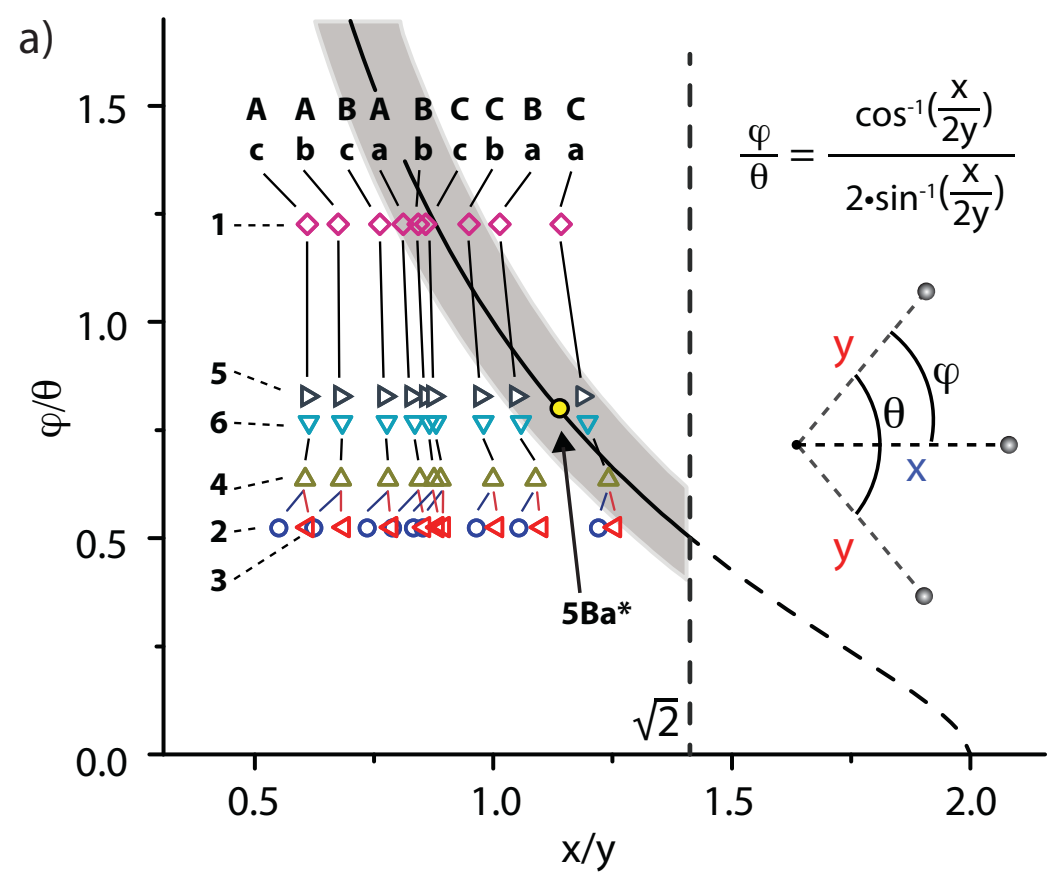

b)

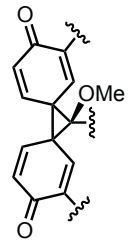

1

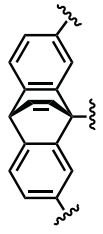

4

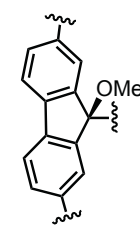

2

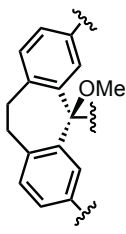

5

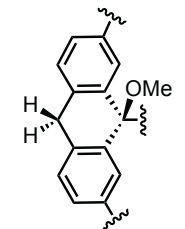

3

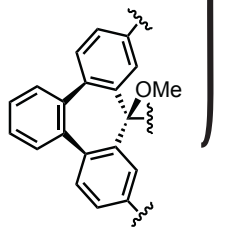

6

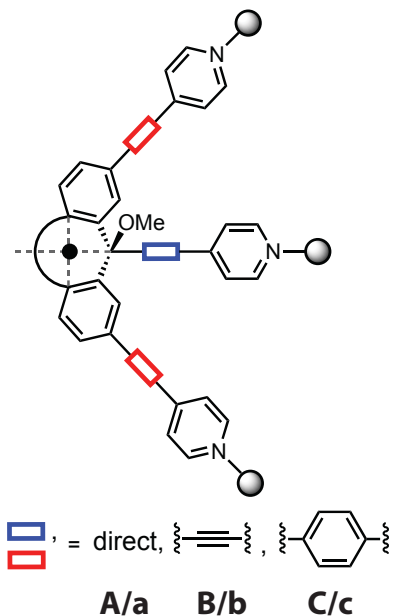

A/a B/b C/c

Figure 4-3 a) Plot of the function $\varphi / \theta=\mathrm{f}(x / y)$, which describes the ideal combination of arm lengths-ratio $x / y$ and angles-ratio $\varphi / \theta$ for the formation of any square-cuboid box with side lengths-ratio $a / c$. The upper limit $x / y=\sqrt{2}$ is marked by a dashed line. b) Selection of tripodal ligands under alteration of the arm lengths $(x$ and $y)$ and the angles $(\varphi$ and $\theta$ ). The backbones 1-6, $x$-arms A-C, and $y$-arms a-c constitute 54 possible ligand structures with different $\varphi / \theta ; x / y$ combinations (marked by different symbols in a). Value 5Ba* has been extracted from a DFT model of the square-cuboid box based on ligand 5Ba $=\mathbf{L}^{2}$. Copyright (C) 2014 WILEY-VCH Verlag GmbH \& Co.

Next, computational screening on a variety of synthetically plausible tripodal ligand structures was carried out, which contain different chemical functionalities at different positions, giving independent variation of the $x / y$ and the $\varphi / \theta$ values (Figure 4-3b). All the ligand backbones were formulated from a tricyclic system with different central ring 
sizes (three-, five-, six-, or seven-membered ring). The pyridine donor sites were linked to the tricyclic system without any linker or with an alkynyl- or with 1,4-phenylene spacer to achieve the different arm lengths. A density functional theory (DFT) geometry optimization of each structure in Figure 4-3b was conducted at B3LYP/6-31G(d,p) level to obtain the $x, y, \varphi$ and $\theta$ values (3.6.6.2).

a)

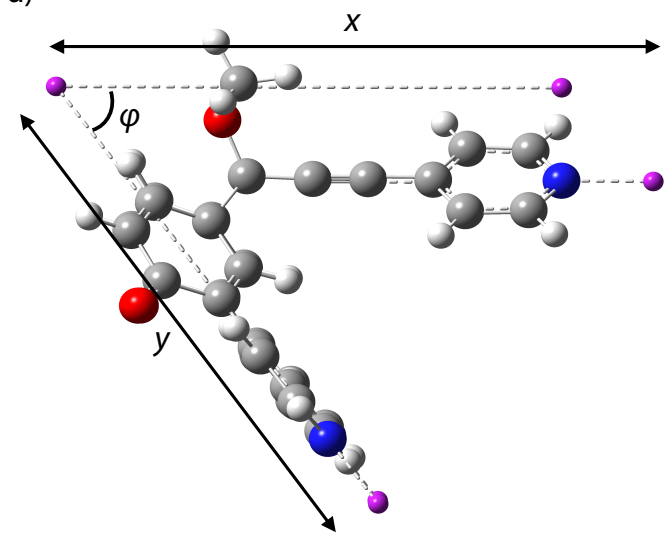

c)

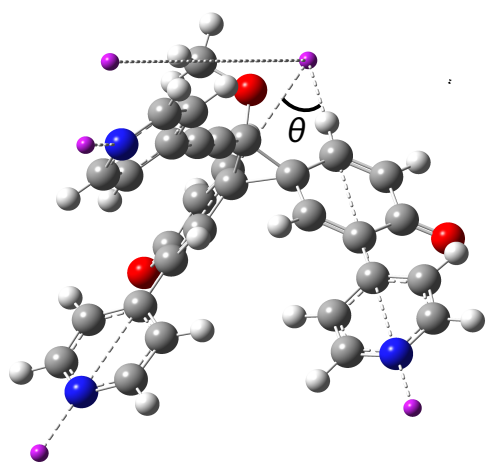

b)

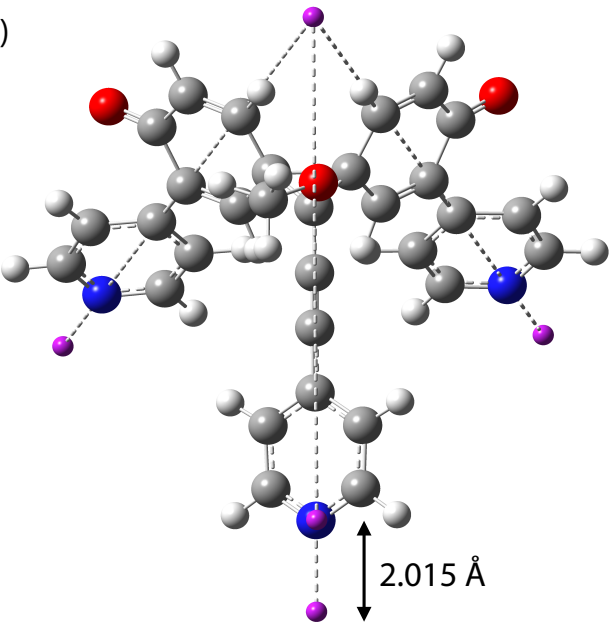

d)

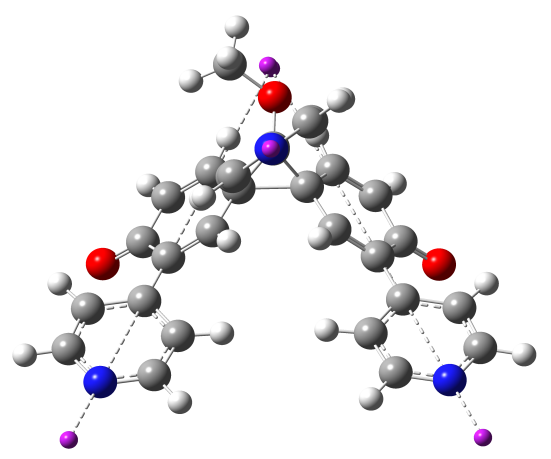

Figure 4-4 Example (1Ba) of the modelling of ligands Backbone 1-6 $_{\text {X }} X$ arm $_{\mathrm{A}-\mathrm{c}} Y$-arma-c. Dummy atoms are depicted in purple. The dummy atoms connected directly to the $\mathrm{N}$-atom in pyridine rings are placed at the position of PdII cation. The distances $x$ and $y$, the angles $\varphi$ and $\theta$ are showed.

A dummy atom was set at the crossing point of the two lines, which extended along the $\mathrm{N}-\mathrm{C}_{\text {ipso }}$ of the pyridine rings of both side arms. The $y$-value was extracted by adding a N-Pd distance $(2.015 \AA)$ to the distance from the dummy atom to the pyridine- $\mathrm{N}$ atom of the side arm (Figure 4-4a,b). $\Theta$ was the angle between the two side arms originated from the vertex (dummy atom). The line along $\mathrm{N}-\mathrm{C}_{\text {ipso }}$ of the central-arm pyridine ring did not intersect with the dummy atom, rather it was then shifted parallel till it meet with the other two lines at the dummy atom. The $x$-value was calculated through addition of the distance between the pyridine- $\mathrm{N}$ atom of the central arm and the dummy atom plus a same N-Pd distance $2.015 \AA . \varphi$ is the angle between the line along one side-arm and the 
line parallel to the central-arm (Figure 4-4c). The combinations $(x / y ; \varphi / \theta)$ of all calculated structures were placed into the graph (Figure 4-3a). 10 of the 54 tentative ligands exhibit possible $x / y ; \varphi / \theta$ combinations located closed to the curve within an arbitrarily chosen range (gray region in Figure 4-3a), which are suitable for the square-cuboid cage formation. We decided to pay particular attention to the experimental work on the sevenmembered dibenzosuberone backbone $\mathbf{5}$, because of the synthetic familiarity to our previous work.[44-47]

\subsection{Ligand design and synthesis}

First, a simple bis-pyridyl ligand $\mathbf{L}^{\mathbf{1}}$ was designed, in which two donor binding sites are arranged at an angle of approximately $90^{\circ}$ (Figure 4-5). As expected, the reaction of the ligand $\mathbf{L}^{1}$ with square-planar coordinating $\left[\mathrm{Pd}\left(\mathrm{CH}_{3} \mathrm{CN}\right)_{4}\right]\left(\mathrm{BF}_{4}\right)_{2}$ in $\mathrm{CD}_{3} \mathrm{CN}$ at a $2: 1$ molar ratio resulted in the quantitative formation of a face-centred cubic $\operatorname{Pd}_{6} \mathbf{L}_{12}$ cage (Figure 4-1a).
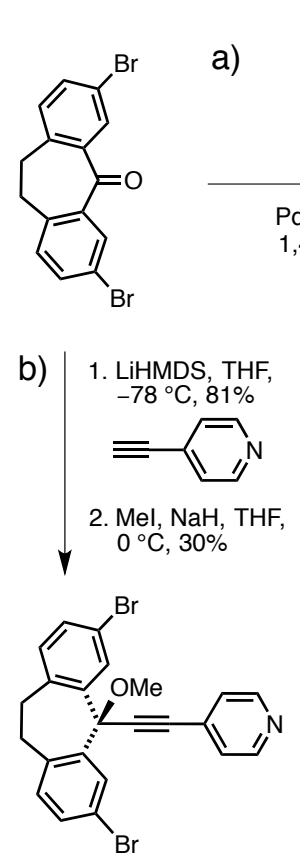

a)

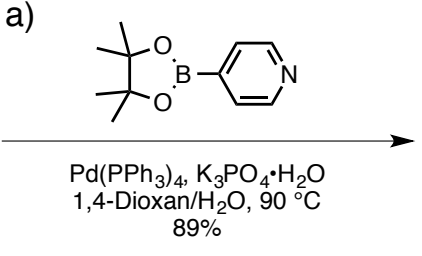

$89 \%$

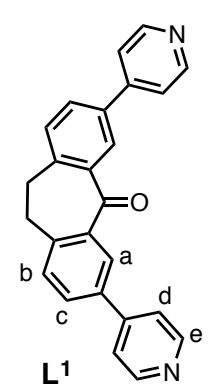

$L^{1}$

rar


FTICR mass spectrum of the coordination cage contained peaks corresponding to the species $\left[\mathrm{Pd}_{6} \mathbf{L}_{12}+n B F_{4}\right]^{(12-n)+}(n=5-7)$ (Figure 4-6). The experimentally observed isotope patterns of the peaks are consistent with the respective calculated values.
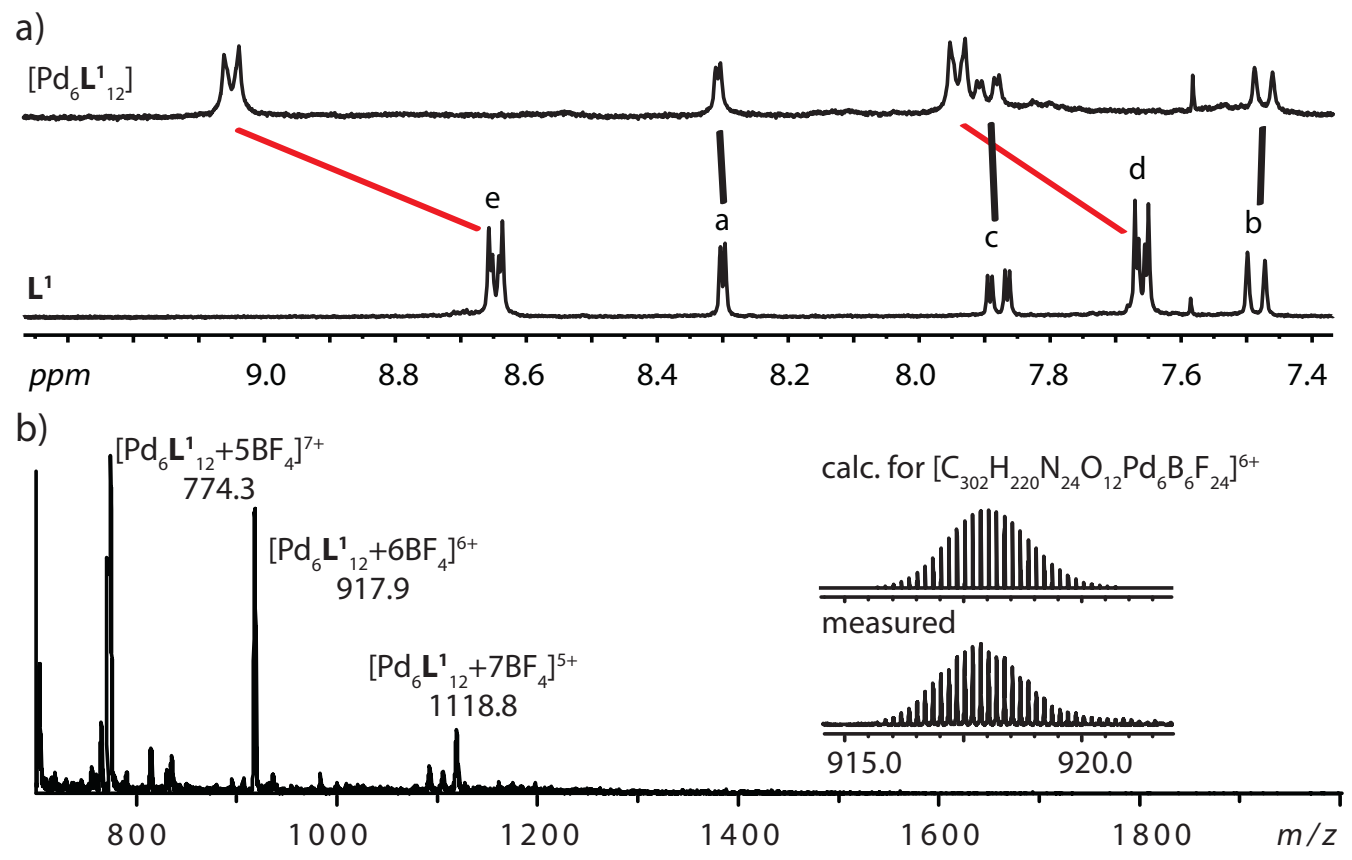

Figure 4-6 a) ${ }^{1} \mathrm{H}$ NMR spectra $\left(300 \mathrm{MHz}, \mathrm{CD}_{3} \mathrm{CN}, 298 \mathrm{~K}\right)$ of ligand $\mathbf{L}^{1}$ and the cubic coordination cage $\operatorname{Pd}_{6} \mathbf{L}_{12}$ and b) ESI-FTICR mass spectrum of the cubic cage $\operatorname{Pd}_{6} \mathbf{L}_{12}$.

Following this success, a third arm was attached to obtain a tripodal ligand $\mathbf{L}^{2}$ (structure 5Ba in Figure 4-5b). 4-ethynylpyridine was first deprotonated using lithium hexamethyldisilazide (LiHMDS) and introduced to 3,7-dibromodibenzosuberone (Figure 4-5b). Alkylation with methyl iodide was performed to protect the resulting tertiary alcohol before a Suzuki cross-coupling was employed to attach the pyridine side arms.

The ${ }^{1} \mathrm{H}$ NMR spectrum of ligand $\mathbf{L}^{2}$ shows recognizable signal shifts for the side arm pyridine protons $\left(\mathrm{H}_{\mathrm{d}}\right.$ and $\left.\mathrm{H}_{\mathrm{e}}\right)$ and the central arm pyridine protons $\left(\mathrm{H}_{\mathrm{f}}\right.$ and $\left.\mathrm{H}_{\mathrm{g}}\right)$ in an integral ratio of $2: 1$ (Figure 4-7a). The ligand $\mathbf{L}^{2}$ was treated with 0.75 equivalents of the same palladium salt $\left[\mathrm{Pd}\left(\mathrm{CH}_{3} \mathrm{CN}\right)_{4}\right]\left(\mathrm{BF}_{4}\right)_{2}$ in $\mathrm{CD}_{3} \mathrm{CN}$, a single species was formed in which all pyridine proton signals were significantly shifted downfield, which is characteristic of metal-ligand coordination. No further signal splitting was observed, which indicates all the donor sites of the tripodal ligand $\mathbf{L}^{2}$ and all the ligands are coordinated with $\mathrm{Pd}^{\mathrm{II}}$, no pyridine donors are dangling uncoordinated. No desymmetrization occurred between the ligand arms, because all the ligands adopt equal positions during the assembly process. The high resolution ESI-MS spectrum further supports the structure of the square-cuboid $\mathrm{Pd}_{6} \mathbf{L}^{2}{ }_{8}$ by revealing a series of dominant peaks corresponding to $\left[\mathrm{Pd}_{6} \mathbf{L}^{2}{ }_{8}+n B F_{4}\right]^{(12-n)+}(n=$ 
5-8). These peaks were isotopically resolved and agreed very well with their calculated distribution (Figure 4-7b).

a)

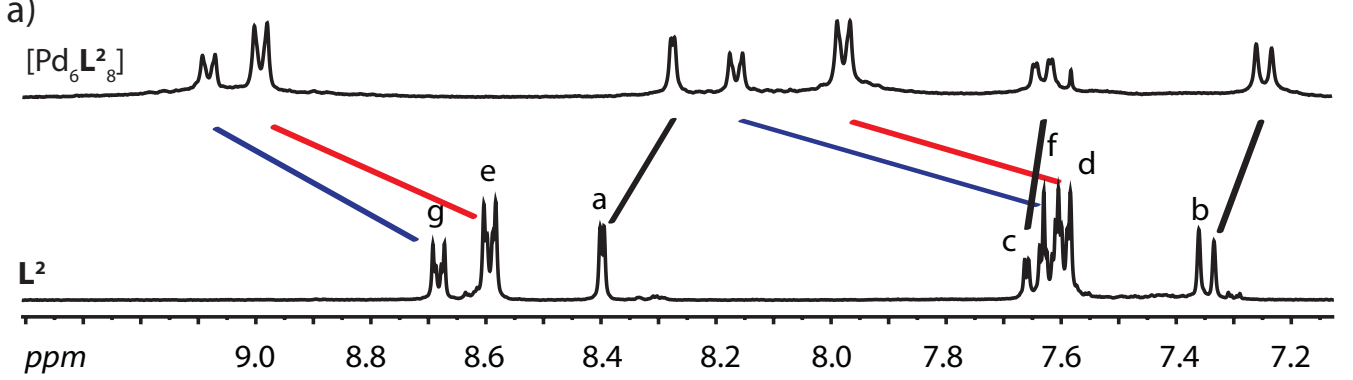

b)

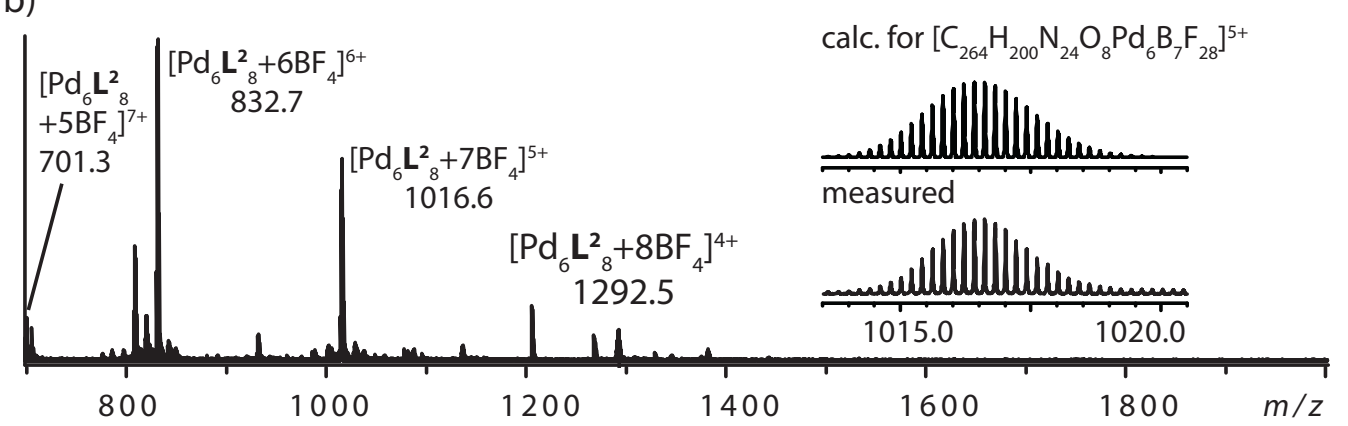

Figure 4-7 a) ${ }^{1} \mathrm{H}$ NMR spectra (300 MHz, $298 \mathrm{~K}$ ) of ligand $\mathbf{L}^{2}$ and the square-cuboid coordination cage $\operatorname{Pd}_{6} \mathbf{L}_{8}$ and b) ESI-MS spectrum of the square-cuboid $\operatorname{Pd}_{6} \mathbf{L}_{8}$ in $\mathrm{CD}_{3} \mathrm{CN}$.

It is notable that a stepwise addition of the $\mathrm{Pd}^{\mathrm{II}}$ salt was required to obtain the best results in terms of solubility and purity of the reaction solution as observed by NMR spectroscopy. A clean NMR spectrum of the complex $\operatorname{Pd}_{6} \mathbf{L}_{8}{ }_{8}$ could then be attained by first adding 0.5 equivalents of $\mathrm{Pd}^{\text {II }}$ to the tripodal ligand $\mathbf{L}^{2}$ solution to form a $\mathrm{Pd}_{4} \mathbf{L}^{2}{ }_{8}$ intermediate complex, followed by another 0.25 equivalents of $\mathrm{Pd} \mathrm{d}^{\mathrm{II}}$ (Figure 4-8).

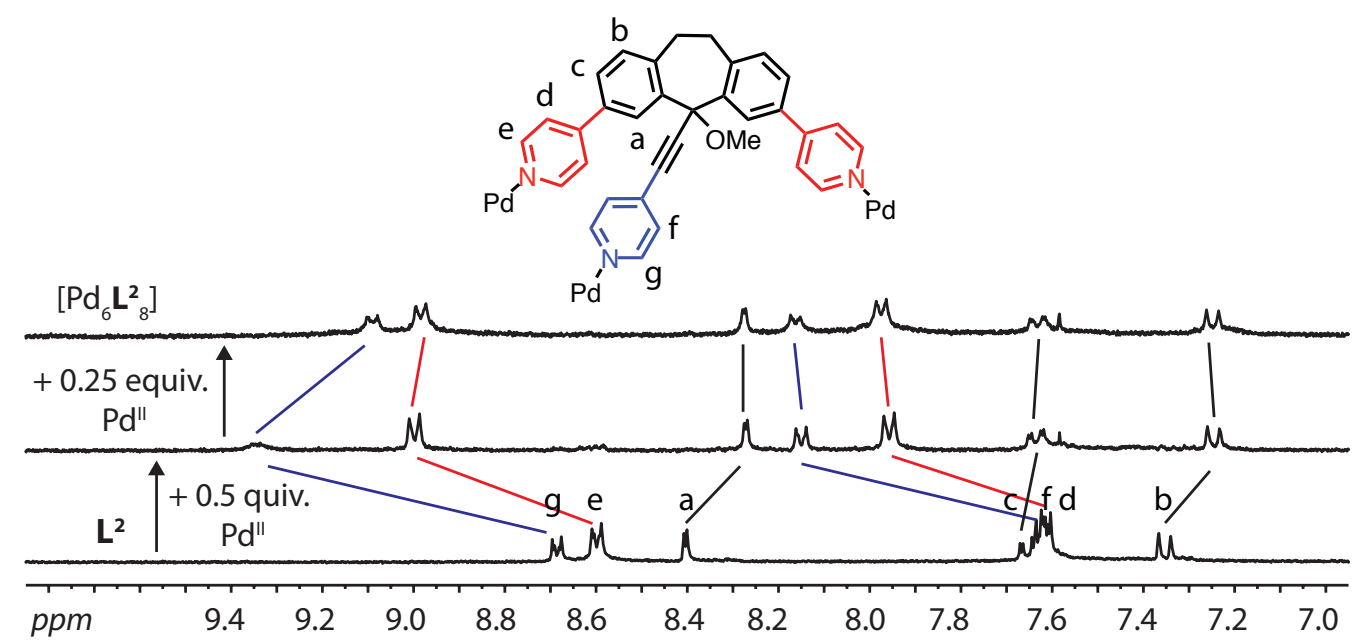

Figure 4-8 $\mathrm{The}^{1} \mathrm{H}$ NMR spectra $\left(300 \mathrm{MHz}, \mathrm{CD}_{3} \mathrm{CN}\right)$ of the stepwise addition of $\mathrm{Pd}^{\mathrm{II}}$ into the ligand $\mathbf{L}^{2}$. 


\subsection{Molecular modelling}

Unfortunately, efforts to grow single crystals of the cage $\mathrm{Pd}_{6} \mathbf{L}^{2}{ }_{8}$ were unsuccessful. However, the single crystals of the free tripodal ligand $\mathbf{L}^{2}$ were obtained by slow evaporation of a solution of $\mathbf{L}^{2}$ in acetonitrile at room temperature (Figure 4-9a). The ligand itself does not form a perfect tripod, as the strained seven-membered ring core leaves the in peripheral pyridine arms and the central pyridine arm slightly distorted. In particular, the central alkynylpyridine arm of $\mathbf{L}^{2}$ is found in a pseudo-equatorial position, which is important for the formation of the $\mathrm{Pd}_{6} \mathbf{L}_{8}{ }_{8}$ assembly, while the methoxy group is pseudo-axially oriented. Comparing the conformation of ligand $\mathbf{L}^{2}$ to our previously reported ligand $\mathbf{L}^{3,[46]}$ which was constructed with the same seven-membered dibenzosuberone backbone, but contains a central methoxylated aryl substituent, instead of the akynylpyridine and a hydroxyl group instead of methoxy group (Figure 4-9b). In this case, the methoxylated aryl arm was found pseudo-axial and the hydroxyl group in a pseudo-equatorial position. DFT calculations on the B3LYP/6-31G+(d) level of theory[64] were carried out of both ligands $\mathbf{L}^{2}$ and $\mathbf{L}^{3}$ to support our experimental discovery. Both ligand structures were simplified before running the calculation. Ethynyl was used as the $x$-arm for $\mathbf{L}^{2}$ instead of ethynylpyridine, and a simple phenyl ring was used to replace the methoxylated aryl x-arm. Side arms were not considered. The relative conformer energies of both ligands were calculated:

$$
\begin{aligned}
& E\left(\mathbf{L}^{2}{ }_{\mathrm{OH}, \text { x arm-eq }}\right)-E\left(\mathbf{L}^{2}{ }_{\mathrm{OH}, \text { x arm-ax }}\right)=+3.3 \mathrm{~kJ} \cdot \mathrm{mol}^{-1} \\
& E\left(\mathbf{L}^{2}{ }_{\text {OMe, } x \text { arm-eq }}\right)-E\left(\mathbf{L}^{2} \text { OMe, } x \text { arm-ax }\right)=-4.1 \mathrm{~kJ} \cdot \mathrm{mol}^{-1} \\
& E\left(\mathbf{L}^{3}{ }_{\mathrm{OH}, \text { x arm-eq }}\right)-E\left(\mathbf{L}^{3} \mathrm{OH}, \text { x arm-ax }\right)=+18.0 \mathrm{~kJ} \cdot \mathrm{mol}^{-1} \\
& E\left(\mathbf{L}^{3}{ }_{\text {OMe, } x \text { arm-eq }}\right)-E\left(\mathbf{L}^{3} \text { OMe, } \text { x arm-ax }\right)=+13.0 \mathrm{~kJ} \cdot \mathrm{mol}^{-1}
\end{aligned}
$$

(The subscripts eq and ax indicate the position of the alkynyl-substituent.) 

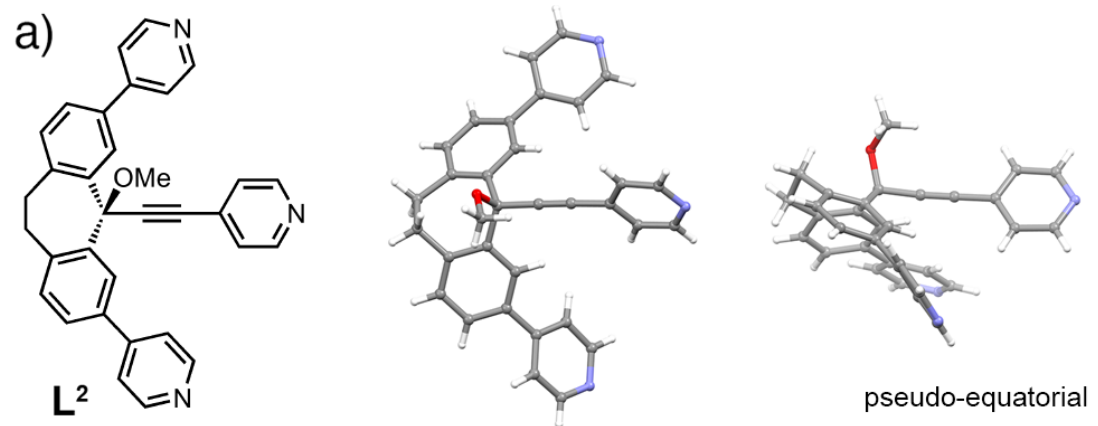

pseudo-equatorial
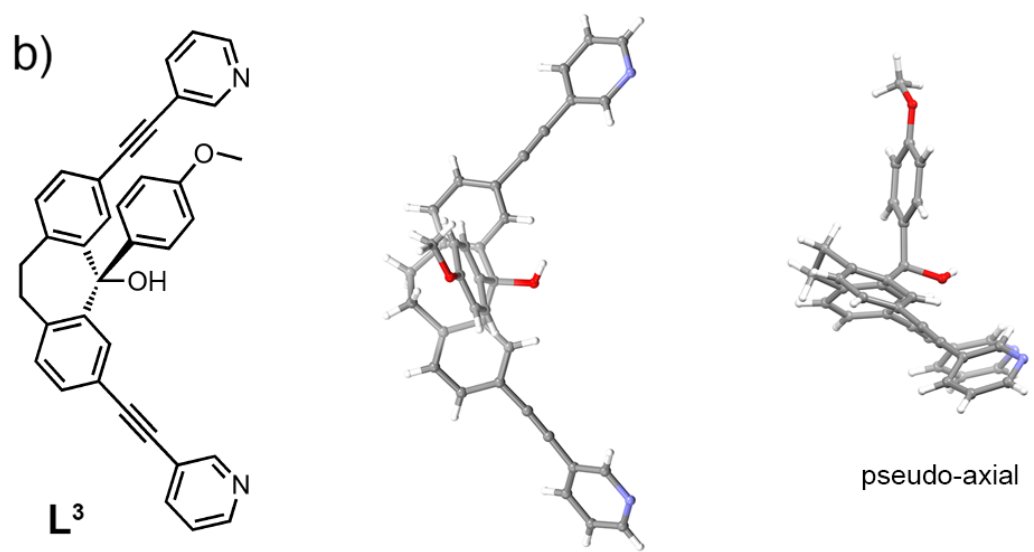

Figure 4-9 X-ray structures of a) ligand $\mathbf{L}^{2}$ with the central alkynyl substituent in a pseudo-equatorial position and b) the related ligand structure $\mathbf{L}^{3}$ in which the central aryl substituent adopts a pseudo-axial conformation (the structure of $\mathbf{L}^{3}$ was extracted from the X-ray structure of the previously reported double cage). Copyright (C) 2014 WILEYVCH Verlag GmbH \& Co.

The theoretical calculations were in good agreement with the experimental results. The calculated results reveal that the pseudo-equatorial position of the alkynyl substituent in $\mathbf{L}^{2}$ is more stable than the pseudo-axial position by $4.1 \mathrm{~kJ} \cdot \mathrm{mol}^{-1}$ whereas the pseudo-axial position of the aryl substituent in $\mathbf{L}^{3}$ is $18.0 \mathrm{~kJ} \cdot \mathrm{mol}^{-1}$ lower in energy and thus the favoured conformation. The DFT calculations also indicate that the oxygen-alkylation has almost no influence on the formation of the conformer. In contrast, only the pseudo-equatorial isomer of oxygen-alkylated ligand $\mathbf{L}^{2}$ are energetically favourable, unalkylated $\mathbf{L}^{\mathbf{2}}$ presents the opposite priority of isomer formation. 
a)

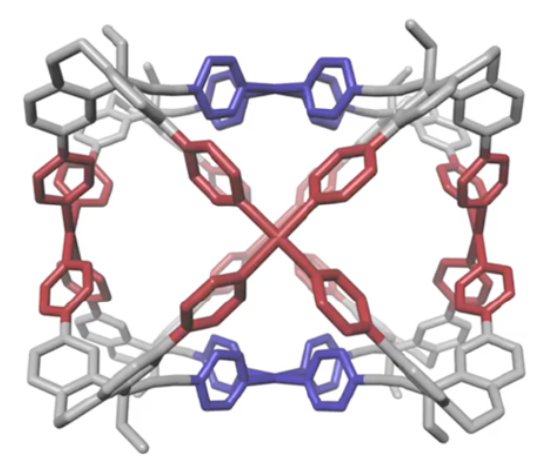

c)

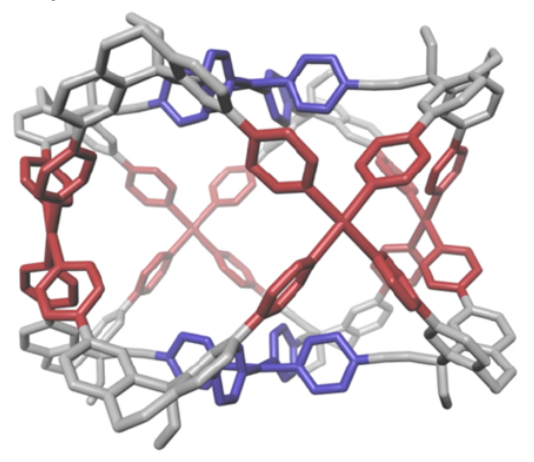

b)

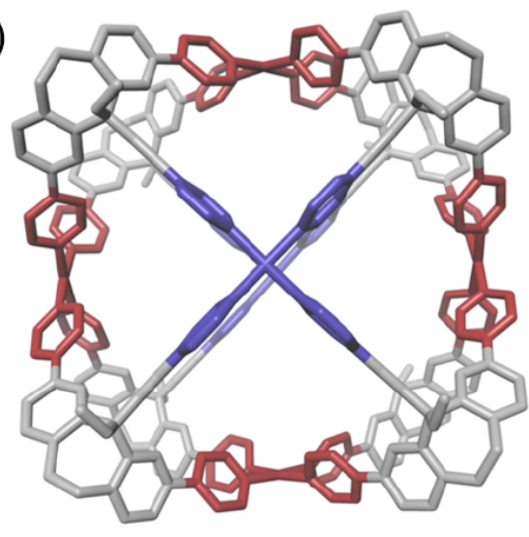

d)

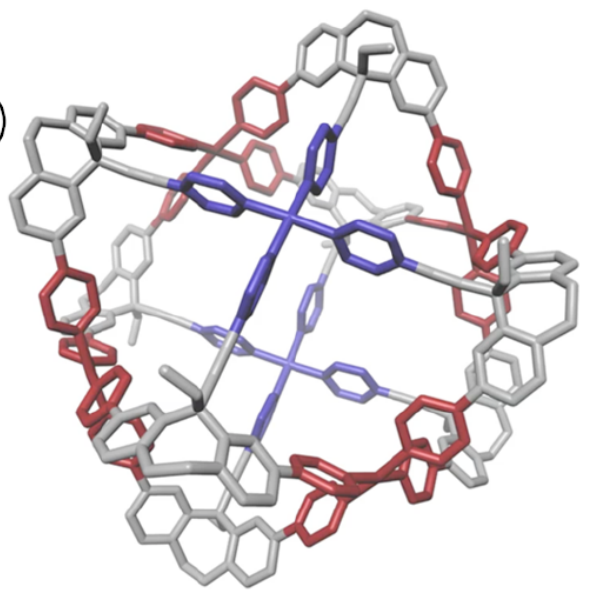

Figure 4-10 Different views of the structure calculated for coordination cage $\left[\operatorname{Pd}_{6} \mathbf{L}_{8}{ }_{8}\right]$. a) Side view, b) top/bottom view, c) and d) arbitrary perspectives of the square-cuboid cage structure. Copyright (C) 2014 WILEY-VCH Verlag GmbH \& Co.

After performing a semiempirical PM6 geometry optimization, the molecular structure of cage $\operatorname{Pd}_{6} \mathbf{L}_{8}{ }_{8}$ was modelled using DFT calculation on a B3LYP/LANL2DZ level of theory.[64] The optimized structures from both methods were compared, however the difference between them was not distinct (Table 4-1):

Table 4-1 Extracted values from PM6 and DFT (B3LYP/LANL2DZ) calculations.

\begin{tabular}{|c|c|c|c|c|}
\hline & $\begin{array}{c}\text { Pd-Pd-distance } \\
\text { along } \boldsymbol{a}(\boldsymbol{b})\end{array}$ & $\begin{array}{c}\text { Pd-Pd-distance } \\
\text { along } \boldsymbol{c}\end{array}$ & $\begin{array}{c}\text { N-Pd-N angle on } \\
\text { red faces }\end{array}$ & $\begin{array}{c}\text { N-Pd-N angle on } \\
\text { blue faces }\end{array}$ \\
\hline PM6 & $19.32 \AA$ & $13.83 \AA$ & $175.8^{\circ}$ & $174.9^{\circ}$ \\
\hline DFT & $19.04 \AA$ & $13.85 \AA$ & $177.1^{\circ}$ & $175.7^{\circ}$ \\
\hline
\end{tabular}


From the DFT calculation, all the ligands sit at the corner of the perfectly formed squarecuboid $\operatorname{Pd}_{6} \mathbf{L}_{8}{ }_{8}$ structure, whose central arm stays in a pseudo-equatorial position. The cage assembly provides two square top and bottom faces (blue in Figure 4-10), which are parallel to each other, and four rectangular side faces (red in Figure 4-10). The values of angles $\varphi$ and $\theta$ extracted directly from DFT optimization exhibit a slight deviation from the values of the calculated free ligand $\mathbf{L}^{2}$ (5Ba in Figure 4-3) as well as from the X-ray structure of free ligand $\mathbf{L}^{2}$ (Figure 4-9a). The probable explanation for this is that the seven-membered ring of the tricyclic backbone could be slightly distorted or twisted because of its flexibility upon assembly of the cage structure. As expected, the arm-length and angle ratio combination $x / y ; \varphi / \theta(x / y=1.14$ and $\varphi / \theta=0.80)$, which were extracted from the DFT optimized structure, lie exactly on the curve we generated according to the mathematical deviation (yellow dot in Figure 4-3a). Through the $x / y ; \varphi / \theta$ combination, the side lengths of the square-cuboid cage $\operatorname{Pd}_{6} \mathbf{L}^{2}, a=b=19.2 \AA$ and $c=14.1 \AA$, could be easily obtained. A self-assembled cage complex with these dimensions should be capable of encapsulating large guests. Therefore a variety of molecules were examined as possible guests (Table 4-4 in 3.6.6), however, no encapsulation was observed by ${ }^{1} \mathrm{H}$ NMR spectroscopy and ESI-MS with any of the investigated molecules. Although the cavity of the square-cuboid should be large enough to fit some guests inside its cavity, as can be inferred from here, the large pores of the cuboid reduce drastically the total solventaccessible surface area, which should be covered upon guest binding, as the amount of solvent accessible surface is a important factor that would interfere the binding strength with host-guest complexation. ${ }^{[48]}$

\subsection{Conclusion}

The mathematical derivation of the dimensions (arm lengths and angles) revealed which conditions a tripodal ligand should obey in order to lead to the desired face-centred square-cuboid cage. We have synthesized a $90^{\circ}$-angled bis-pyridyl ligand $\mathbf{L}^{1}$ which gave rise to a cubic coordination cage $\operatorname{Pd}_{6} \mathbf{L}_{12}$. Following the same geometric considerations a tripodal tris-pyridyl ligand $\mathbf{L}^{2}$ was designed and synthesized from a common dibenzosuberone precursor, and the structure of $\mathbf{L}^{\mathbf{2}}$ was confirmed by X-ray crystallography. Although no single crystal X-ray data was obtained for both cage complexes, 1D and 2D NMR spectroscopy, ESI mass spectrometry, and DFT modelling all supported the formation of the proposed structures.

Interestingly, some face-centred coordination cages with same metal-ligand ratio $\mathbf{M}_{6} \mathbf{L}_{8}$ have been published, ${ }^{[49-51]}$ although these examples were built using $C_{3}$-symmetric pyramidal shaped ligands, where the three binding sites of these ligands are not 
distinguishable, meaning $x / y=1$, and $\varphi / \theta=1$ (Figure 4-3). However, as designed, we were able to generate the first example of a homonuclear $D_{4 h}$-symmetric square-cuboid coordination cage $\operatorname{Pd}_{6} \mathbf{L}^{2}{ }_{8}$, which was composed by eight $C_{s}$-symmetric tripodal ligands and six square planar PdII cations. All six faces (two square top and bottom faces, and four rectangular side faces) were constructed using simple Pd(pyridine) ${ }_{4}$ complexes, without any heteronuclear or heteroleptic coordination approach.

We anticipate that the information gleaned from the design and analysis of the assembly processes presented here will be promising for the rational synthesis of complex reduced symmetry molecular architectures.[52]

\subsection{Experimental section}

\subsubsection{Synthesis of ligand $\mathbf{L}^{\mathbf{1}}$ and $\mathbf{L}^{\mathbf{2}}$}

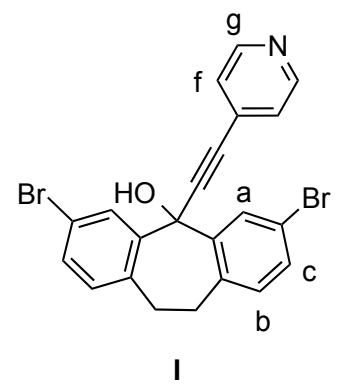

A 1 M solution of LiHMDS in dry THF ( $5.5 \mathrm{~mL}, 5.46 \mathrm{mmol}$, 2 equiv.) was added dropwise to a solution of 4-ethynylpyridine ${ }^{[53]}(0.71 \mathrm{~g}, 6.83 \mathrm{mmol}, 2.5$ equiv.) in dry THF (40 mL) under vigorous stirring at $-78^{\circ} \mathrm{C}$. The mixture was stirred for $2 \mathrm{~h}$ at this temperature before 3,7-dibromodibenzo-suberone[54] (1.00 g, $2.73 \mathrm{mmol}, 1$ equiv.) in dry THF (15 mL) was added slowly. The solution temperature was maintained at $-78{ }^{\circ} \mathrm{C}$ for 30 min then allowed to warm to room temperature and stirred overnight. The reaction was quenched by the addition of water and extracted with diethyl ether. The organic layer was collected, dried over $\mathrm{Na}_{2} \mathrm{SO}_{4}$, filtered and concentrated. The residue was purified by column chromatography (silica gel) using $\mathrm{CHCl}_{3} / \mathrm{MeOH}$ as eluents to give $\mathbf{I}(1.04 \mathrm{~g}, 81 \%)$ as a brown solid.

${ }^{1} \mathbf{H}-\mathbf{N M R}\left(300 \mathrm{MHz}, \mathrm{CDCl}_{3}\right) \delta 3.07-3.17\left(2 \mathrm{H}, \mathrm{m}, \mathrm{CH}_{2}\right), 3.61(1 \mathrm{H}, \mathrm{s}, \mathrm{OH}), 3.59-3.70(2 \mathrm{H}, \mathrm{m}$, $\left.\mathrm{CH}_{2}\right), 7.03\left(2 \mathrm{H}, \mathrm{d}, 3 \mathrm{~J}=8.1 \mathrm{~Hz}, \mathrm{H}_{\mathrm{b}}\right), 7.28\left(2 \mathrm{H}, \mathrm{dd}, 3 J=6.0 \mathrm{~Hz}, 4 J=1.5 \mathrm{~Hz}, \mathrm{H}_{\mathrm{f}}\right), 7.37(2 \mathrm{H}, \mathrm{dd}, 3 J=$ $\left.8.1 \mathrm{~Hz},{ }^{4} J=2.1 \mathrm{~Hz}, \mathrm{H}_{\mathrm{c}}\right), 8.12\left(2 \mathrm{H}, \mathrm{d},{ }^{4} J=2.1 \mathrm{~Hz}, \mathrm{H}_{\mathrm{a}}\right), 8.57\left(2 \mathrm{H}, \mathrm{dd},{ }^{3} J=6.0 \mathrm{~Hz},{ }^{4} J=1.5 \mathrm{~Hz}, \mathrm{H}_{\mathrm{g}}\right)$. 


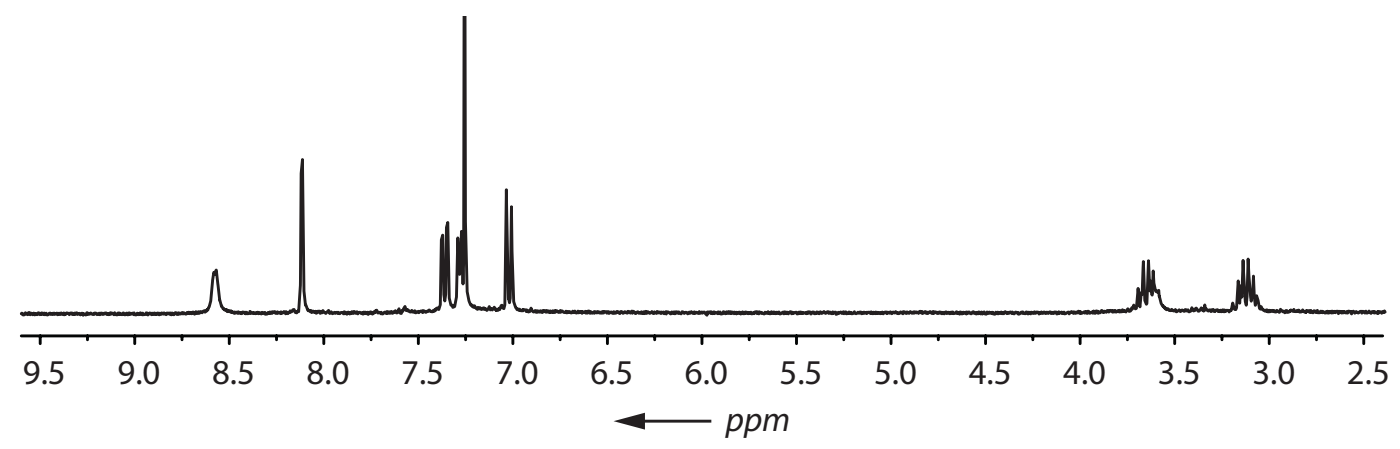

${ }^{13}$ C-NMR $\left(125 \mathrm{MHz}, \mathrm{CDCl}_{3}\right) \delta 31.71,71.01,84.51,95.46,120.26,125.67,127.87,130.24$, $131.54,132.70,136.53,142.55,149.71$.

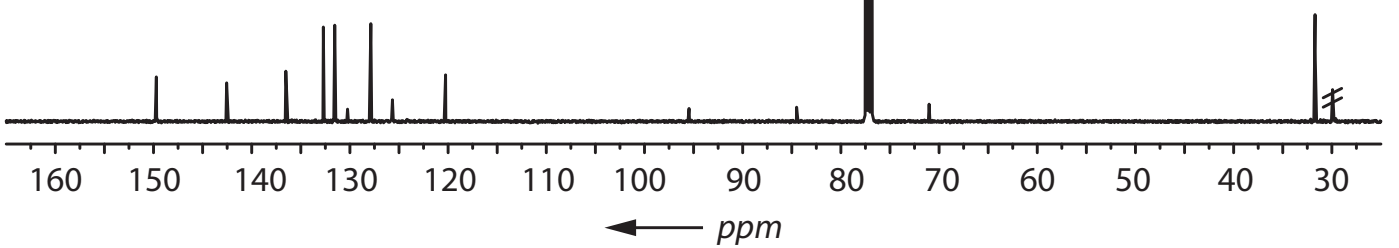

ESI-MS calculated for $\mathrm{C}_{22} \mathrm{H}_{15} \mathrm{Br}_{2} \mathrm{NO}[\mathrm{M}+\mathrm{Na}]^{+} \mathrm{m} / z 491.9$ found $m / z 491.9$.

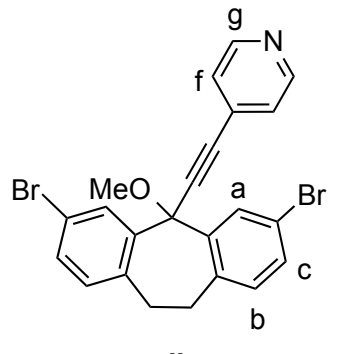

II

A mixture of $60 \% \mathrm{NaH}(0.26 \mathrm{~g}, 6.46 \mathrm{mmol}, 3$ equiv., pre-washed with dry THF to remove mineral oil) and I (1.01 g, 2.15 mmol, 1 equiv., in $15 \mathrm{~mL}$ dry THF) in dry THF (5 mL) was stirred for $2 \mathrm{~h}$ under nitrogen. To the pale brown solution was added Mel $(3.05 \mathrm{~g}$, $21.5 \mathrm{mmol}, 1.35 \mathrm{~mL}, 10$ equiv.) slowly. The mixture was allowed to warm to room temperature, and stirred overnight. Water was added to quench the residual $\mathrm{NaH}$, and the organics were extracted with $\mathrm{CHCl}_{3}$, dried over $\mathrm{MgSO}_{4}$, and the solvent was removed in vacuo. The residue was subjected to column chromatography (silica, $\mathrm{CHCl}_{3} / \mathrm{MeOH}$ ) to afford a brown solid (0.32 g, 30\%).

1H-NMR $\left(300 \mathrm{MHz}, \mathrm{CDCl}_{3}\right) \delta 2.92-3.10\left(2 \mathrm{H}, \mathrm{m}, \mathrm{CH}_{2}\right), 3.40\left(3 \mathrm{H}, \mathrm{s}, \mathrm{OCH}_{3}\right), 3.54-3.69(2 \mathrm{H}, \mathrm{m}$, $\left.\mathrm{CH}_{2}\right), 7.02\left(2 \mathrm{H}, \mathrm{d},{ }^{3} J=8.1 \mathrm{~Hz}, \mathrm{H}_{\mathrm{b}}\right), 7.37\left(2 \mathrm{H}, \mathrm{dd},{ }^{3} J=8.1 \mathrm{~Hz},{ }^{4} J=2.1 \mathrm{~Hz}, \mathrm{H}_{\mathrm{c}}\right), 7.52\left(2 \mathrm{H}, \mathrm{dd},{ }^{3} J=\right.$ $\left.6.0 \mathrm{~Hz},{ }^{4} J=1.5 \mathrm{~Hz}, \mathrm{H}_{\mathrm{f}}\right), 8.11\left(2 \mathrm{H}, \mathrm{d},{ }^{4} J=2.1 \mathrm{~Hz}, \mathrm{H}_{\mathrm{a}}\right), 8.71\left(2 \mathrm{H}, \mathrm{dd},{ }^{3} J=6.0 \mathrm{~Hz},{ }^{4} J=1.5 \mathrm{~Hz}, \mathrm{H}_{\mathrm{g}}\right)$. 


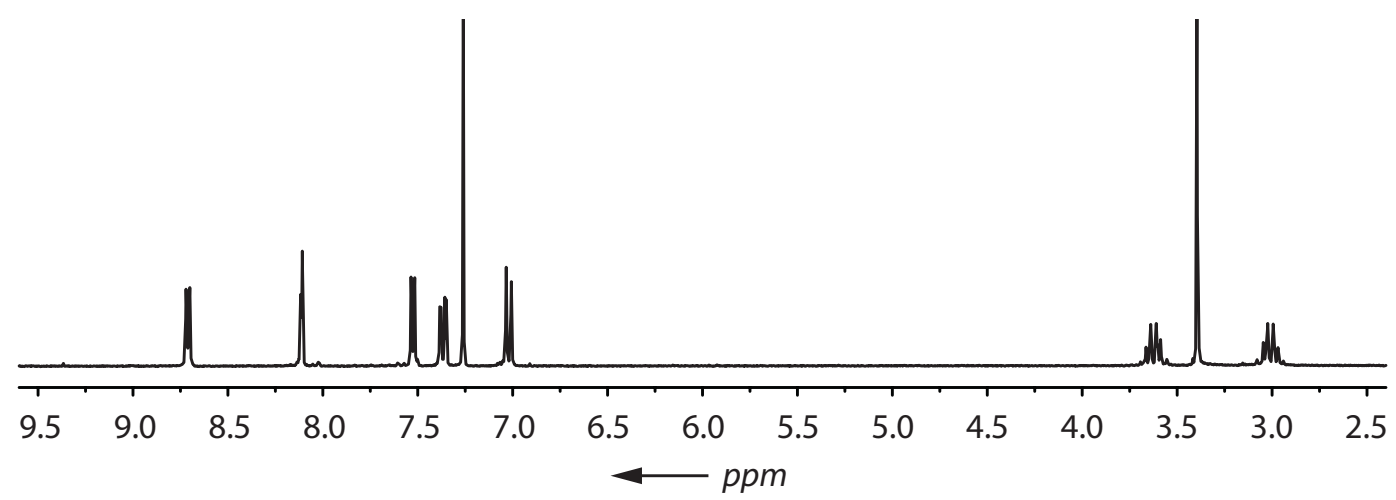

13C-NMR $\left(75 \mathrm{MHz}, \mathrm{CDCl}_{3}\right) \delta 31.54,52.70,83.08,90.44,91.18,119.76,125.91,130.09$, $130.65,132.03,133.32,137.35,139.96,150.19$.

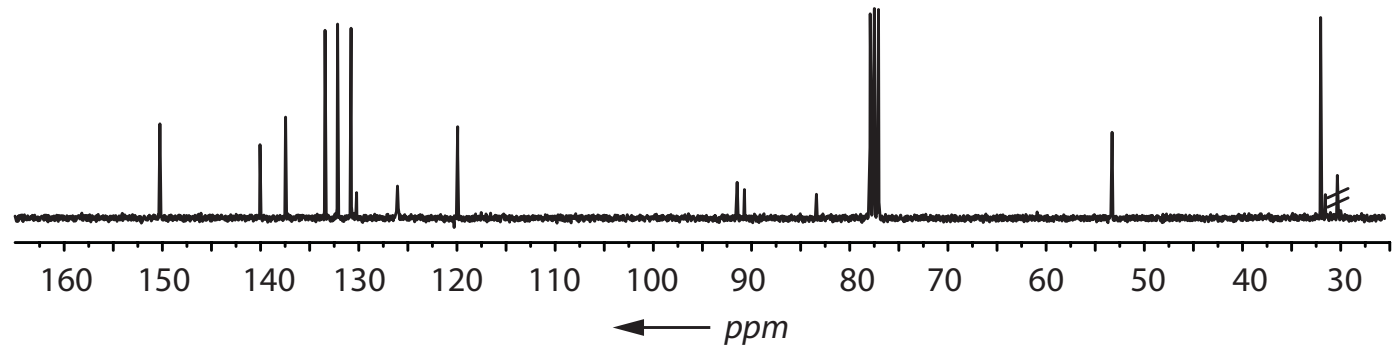

ESI-MS calculated for $\mathrm{C}_{23} \mathrm{H}_{17} \mathrm{Br}_{2} \mathrm{NO}[\mathrm{M}+\mathrm{Na}]^{+} m / z 506.0$ found $m / z 506.0$.

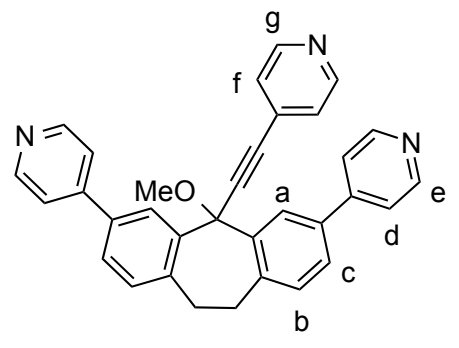

$\mathrm{L}^{2}$

To an oven-dried Schlenk tube was added $\mathrm{Pd}\left(\mathrm{PPh}_{3}\right)_{4}(57.9 \mathrm{mg}, 0.05 \mathrm{mmol}, 10 \mathrm{~mol} \%)$, 4pyridineboronic acid pinacol ester (308.7 mg, $1.51 \mathrm{mmol}, 3$ equiv.) and $\mathrm{K}_{3} \mathrm{PO}_{4} \cdot \mathrm{H}_{2} \mathrm{O}(1.73 \mathrm{~g}$, $7.53 \mathrm{mmol}, 15$ equiv.). The Schlenk tube was evacuated and backfilled with nitrogen (three times) to which degassed distilled water $(2 \mathrm{~mL})$ and 1,4-dioxane $(6 \mathrm{~mL})$ were degassed and added sequentially. The resulting mixture was stirred for $3 \mathrm{~min}$ at room temperature. A solution of II (242.5 mg, $0.50 \mathrm{mmol}, 1$ equiv.) in 1,4-dioxane ( $6 \mathrm{~mL}$ ) was added. The mixture was heated at $90{ }^{\circ} \mathrm{C}$ overnight. Once the conversion was complete (by TLC), the reaction mixture was allowed to cool to room temperature, then the solvent was evaporated. The residue was washed with $\mathrm{CHCl}_{3}$ and $\mathrm{H}_{2} \mathrm{O}$, the organic phase was dried over anhydrous $\mathrm{MgSO}_{4}$ and concentrated in vacuo. The crude product mixture was separated by silica gel column chromatography using $\mathrm{CHCl}_{3} / \mathrm{MeOH}$ (gradient) as eluents to give $\mathbf{L}^{2}$ (208.6 $\mathrm{mg}, 87 \%$ ) as a pale yellow solid. 
1H-NMR (300 MHz, $\left.\mathrm{CDCl}_{3}\right) \delta 3.10-3.27\left(2 \mathrm{H}, \mathrm{m}, \mathrm{CH}_{2}\right), 3.47\left(3 \mathrm{H}, \mathrm{s}, \mathrm{OCH}_{3}\right), 3.72-3.88(2 \mathrm{H}, \mathrm{m}$, $\left.\mathrm{CH}_{2}\right), 7.31\left(2 \mathrm{H}, \mathrm{d}, 3 \mathrm{~J}=7.8 \mathrm{~Hz}, \mathrm{H}_{\mathrm{b}}\right), 7.46-7.52\left(6 \mathrm{H}, \mathrm{m}, \mathrm{H}_{\mathrm{f}}, \mathrm{H}_{\mathrm{d}}\right), 7.54\left(2 \mathrm{H}, \mathrm{dd}, 3 \mathrm{~J}=7.8 \mathrm{~Hz},{ }^{4} \mathrm{~J}=2.1\right.$ $\left.\mathrm{Hz}, \mathrm{H}_{\mathrm{c}}\right), 8.33\left(2 \mathrm{H}, \mathrm{d}, 4 J=2.1 \mathrm{~Hz}, \mathrm{H}_{\mathrm{a}}\right), 8.63\left(4 \mathrm{H}, \mathrm{dd}, 3 J=6.0 \mathrm{~Hz}, 4 J=1.5 \mathrm{~Hz}, \mathrm{H}_{\mathrm{e}}\right), 8.71\left(2 \mathrm{H}, \mathrm{dd},{ }^{3} J\right.$ $=6.0 \mathrm{~Hz},{ }^{4}=1.5 \mathrm{~Hz}, \mathrm{H}_{\mathrm{g}}$ ).

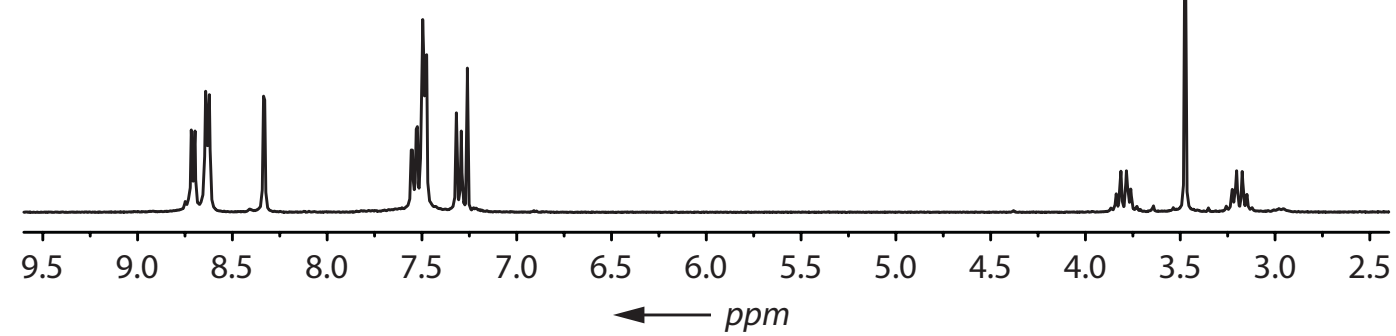

${ }^{13}$ C-NMR $\left(75 \mathrm{MHz}, \mathrm{CDCl}_{3}\right) \delta 32.05,52.87,84.16,90.44,92.04,121.45,125.71,126.34$, $127.46,130.20,132.63,135.97,139.03,139.80,148.03,150.33,150.43$.

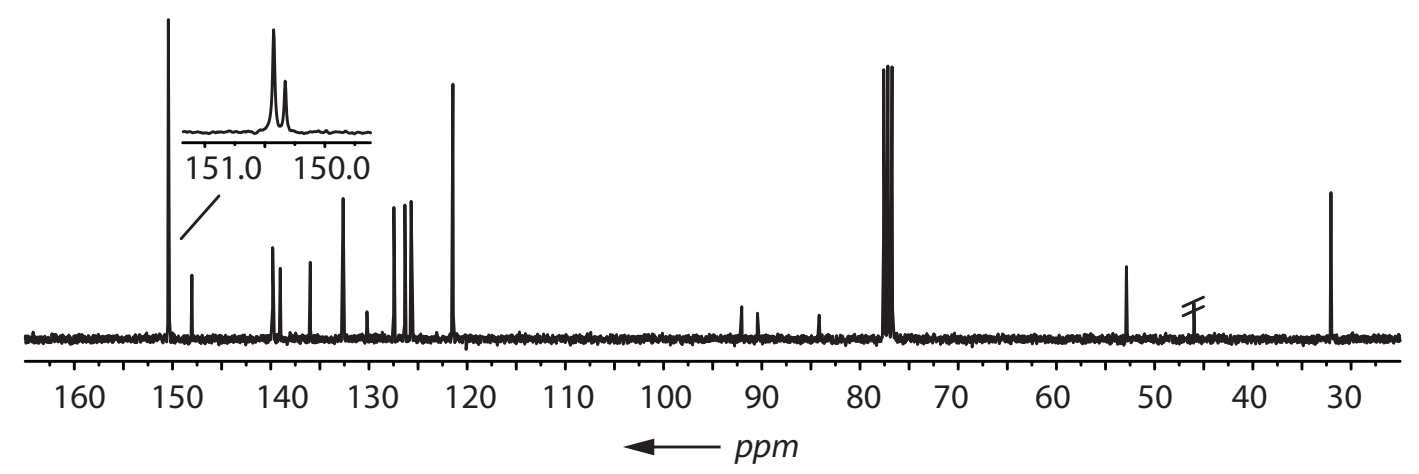

ESI-MS calculated for $\mathrm{C}_{33} \mathrm{H}_{25} \mathrm{~N}_{3} \mathrm{O}[\mathrm{M}+\mathrm{H}]+m / z 480.2$ found $m / z$ 480.2.

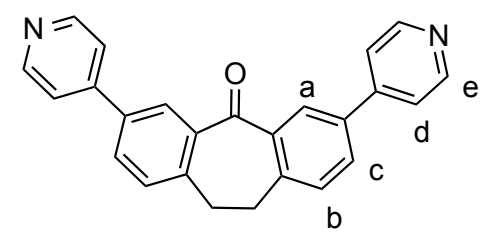

$L^{1}$

Following the procedure of $\mathbf{L}^{2}$, a mixture of $\mathrm{Pd}_{\left(\mathrm{PPh}_{3}\right)_{4}}(69.5 \mathrm{mg}, 10 \mathrm{~mol} \%), 4$ pyridineboronic acid pinacol ester (369.7 mg, $1.82 \mathrm{mmol}, 3$ equiv.), $\mathrm{K}_{3} \mathrm{PO}_{4} \cdot \mathrm{H}_{2} \mathrm{O}$ (2.1 g, $9.01 \mathrm{mmol}, 15$ equiv.) and 3,7-dibromodibenzosuberone (220 mg, $0.60 \mathrm{mmol}, 1$ equiv., in 1,4-dioxane), degassed distilled water $(1.5 \mathrm{~mL})$ and 1,4-dioxane $(7 \mathrm{~mL})$ was stirred at $90{ }^{\circ} \mathrm{C}$ overnight under nitrogen. The product was purified by column chromatography (silica) using $\mathrm{CHCl}_{3} / \mathrm{MeOH}$ (gradient) as eluents to afford $\mathbf{L}^{\mathbf{1}}$ (193.5 $\mathrm{mg}, 89 \%$ ) as a light yellow solid. 
1H-NMR (300 MHz, CD $\left.{ }_{3} \mathrm{CN}\right) \delta 3.31\left(4 \mathrm{H}, \mathrm{s}, \mathrm{CH}_{2}-\mathrm{CH}_{2}\right), 7.49\left(2 \mathrm{H}, \mathrm{d},{ }^{3} J=7.8 \mathrm{~Hz}, \mathrm{H}_{\mathrm{b}}\right), 7.66(4 \mathrm{H}$, $\left.\mathrm{dd}, 3 J=6.0 \mathrm{~Hz},{ }^{4} J=1.5 \mathrm{~Hz}, \mathrm{H}_{\mathrm{d}}\right), 7.88\left(2 \mathrm{H}, \mathrm{dd},{ }^{3} J=7.8 \mathrm{~Hz},{ }^{4} J=2.1 \mathrm{~Hz}, \mathrm{H}_{\mathrm{c}}\right), 8.30\left(2 \mathrm{H}, \mathrm{d},{ }^{4} J=2.1\right.$ $\left.\mathrm{Hz}, \mathrm{H}_{\mathrm{a}}\right), 8.65\left(4 \mathrm{H}, \mathrm{dd}, 3 \mathrm{~J}=6.0 \mathrm{~Hz},{ }^{4} J=1.5 \mathrm{~Hz}, \mathrm{H}_{\mathrm{e}}\right)$.

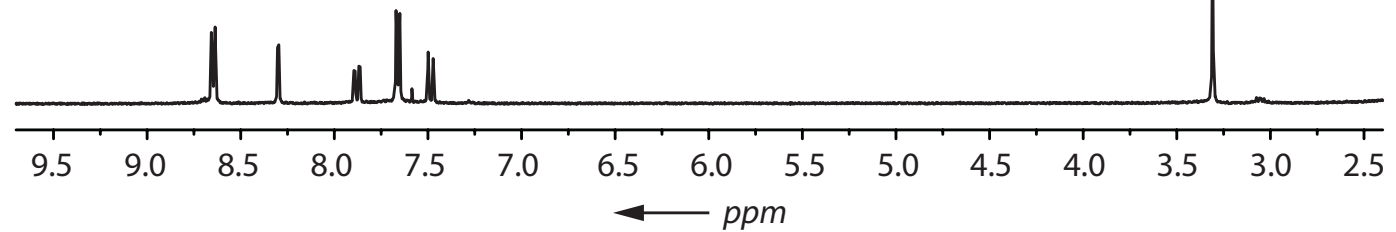

${ }^{13} \mathrm{C}-\mathrm{NMR}\left(125 \mathrm{MHz}, \mathrm{CD}_{3} \mathrm{CN}\right) \delta 35.11,122.28,129.55,131.71,131.78,137.10,139.89$, $144.32,147.79,151.11,195.23$.

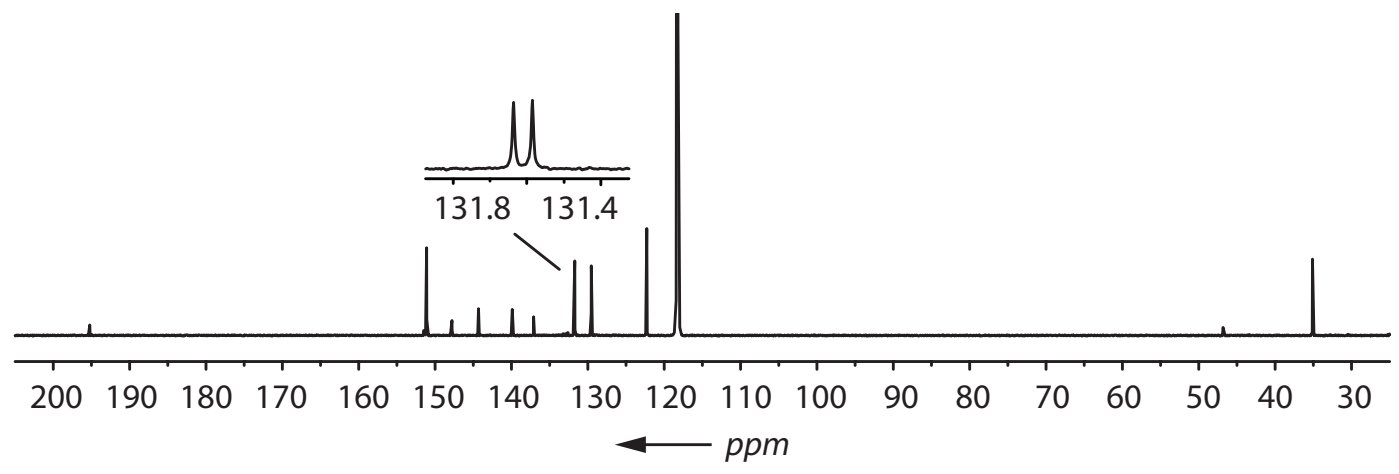

ESI-MS calculated for $\mathrm{C}_{25} \mathrm{H}_{18} \mathrm{~N}_{2} \mathrm{O}[\mathrm{M}+\mathrm{H}]^{+} m / z 363.1$ found $m / z 363.1$.

\subsubsection{Synthesis of $\left[\mathrm{Pd}_{6} \mathbf{L}^{\mathbf{1}_{12}}\right]\left(\mathrm{BF}_{4}\right)_{12}$}

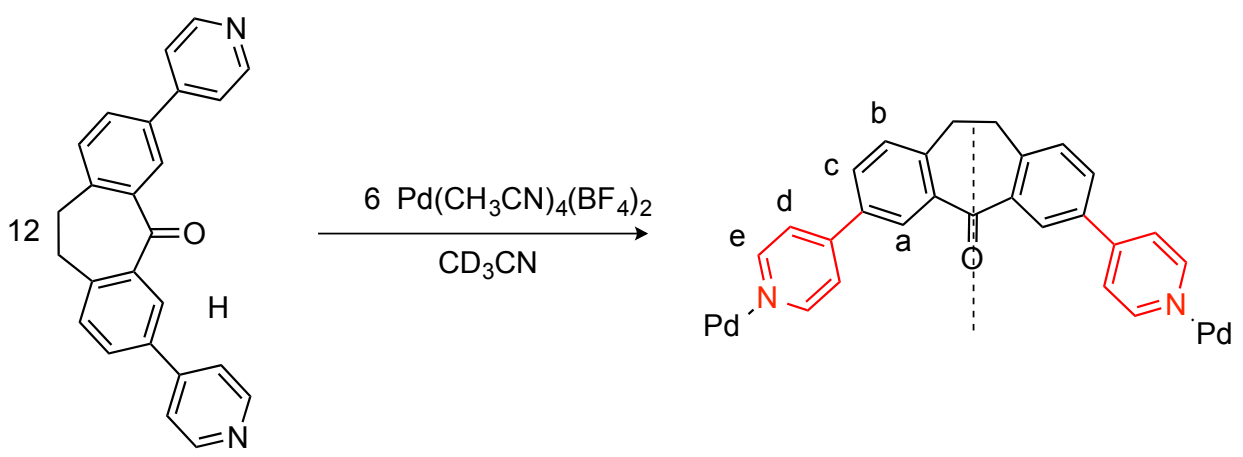

The $\operatorname{Pd}_{6} \mathbf{L}_{12}$ cage compound was prepared by heating a mixture of the ligand $\mathbf{L}^{\mathbf{1}}$ (3.6 $\mu$ mol, $120 \mu \mathrm{L}$ of a $30 \mathrm{~mm}$ stock solution in $\left.\mathrm{CD}_{3} \mathrm{CN}\right)$ and $\left[\mathrm{Pd}\left(\mathrm{CH}_{3} \mathrm{CN}\right)_{4}\right]\left(\mathrm{BF}_{4}\right)_{2}(1.8 \mu \mathrm{mol}, 60 \mu \mathrm{L}$ of a $30 \mathrm{~mm}$ stock solution in $\mathrm{CD}_{3} \mathrm{CN}$ ) in $420 \mu \mathrm{L} \mathrm{CD}{ }_{3} \mathrm{CN}$ at $70{ }^{\circ} \mathrm{C}$ for $1 \mathrm{~h}$ in a closed vial to yield $600 \mu \mathrm{L}$ of a $0.5 \mathrm{mM}$ solution of the cage compound quantitatively.

1H-NMR (300 MHz, CD $\left.{ }_{3} \mathrm{CN}\right) \delta 3.26\left(48 \mathrm{H}, \mathrm{s}, \mathrm{CH}_{2}-\mathrm{CH}_{2}\right), 7.47\left(24 \mathrm{H}, \mathrm{d}, 3 \mathrm{~J}=8.2 \mathrm{~Hz}, \mathrm{H}_{\mathrm{b}}\right), 7.89$ $\left(24 \mathrm{H}, \mathrm{dd}, 3 J=8.2 \mathrm{~Hz},{ }^{4} J=2.1 \mathrm{~Hz}, \mathrm{H}_{\mathrm{c}}\right), 7.94\left(48 \mathrm{H}, \mathrm{dd},{ }^{3} J=6.9 \mathrm{~Hz},{ }^{4} J=1.5 \mathrm{~Hz}, \mathrm{H}_{\mathrm{d}}\right), 8.31(24 \mathrm{H}$, d, $\left.4 J=2.1 \mathrm{~Hz}, \mathrm{H}_{\mathrm{a}}\right), 9.05\left(48 \mathrm{H}, \mathrm{dd}, 3 J=6.9 \mathrm{~Hz},{ }^{4} J=1.5 \mathrm{~Hz}, \mathrm{H}_{\mathrm{e}}\right)$. 


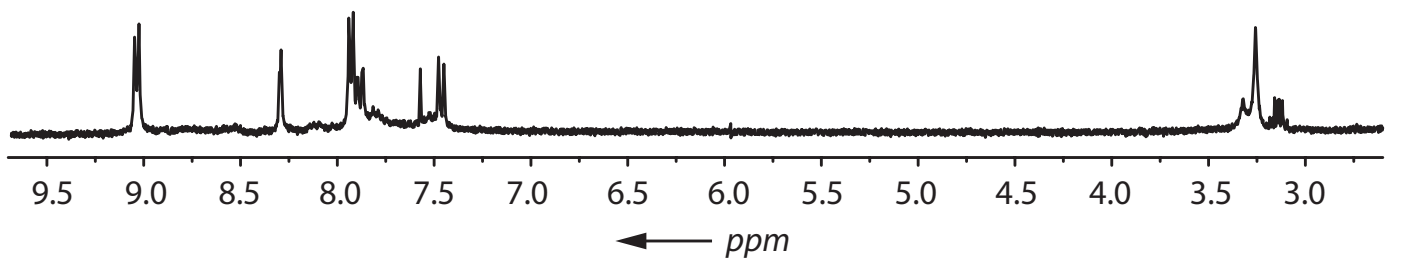

${ }^{13}$ C-NMR $\left(125 \mathrm{MHz}, \mathrm{CD}_{3} \mathrm{CN}\right) \delta 34.78,125.20,129.93,131.85,132.17,134.05,140.06$, 145.96, 151.61, 152.21, 194.93 .

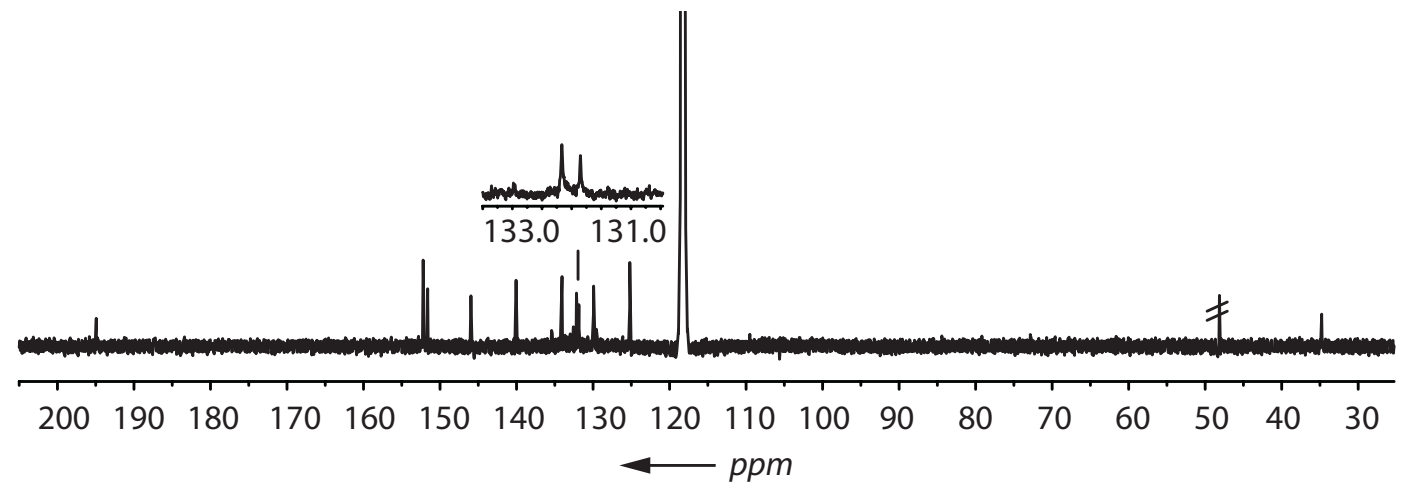

HRMS (ESI, pos.) $m / z\left(\left[\operatorname{Pd}_{6} \mathbf{L}_{12}+7 \mathrm{BF}_{4}\right]^{5+}\right)$ calcd. $=1118.8316$, found $=1118.8323$.

\subsubsection{Synthesis of $\left[\mathrm{Pd}_{6} \mathbf{L}^{2}{ }_{8}\right]\left(\mathrm{BF}_{4}\right)_{12}$}<smiles>[B]C1Cc2ccc(-c3ccncc3)cc2C(C)(C#Cc2ccncc2)c2cc(-c3ccncc3)ccc21</smiles>

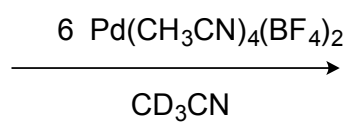<smiles></smiles>

The $\operatorname{Pd}_{6} \mathbf{L}_{8}{ }_{8}$ cage compound was synthesized by first heating a mixture of the ligand $\mathbf{L}^{2}$ $\left(12 \mu \mathrm{mol}, 400 \mu \mathrm{L}\right.$ of a $30 \mathrm{~mm}$ stock solution in $\left.\mathrm{CD}_{3} \mathrm{CN}\right)$ with $\left[\mathrm{Pd}\left(\mathrm{CH}_{3} \mathrm{CN}\right)_{4}\right]\left(\mathrm{BF}_{4}\right)_{2}(6 \mu \mathrm{mol}$, $200 \mu \mathrm{L}$ of a $30 \mathrm{~mm}$ stock solution in $\mathrm{CD}_{3} \mathrm{CN}$ ) in $2300 \mu \mathrm{L} \mathrm{CD} \mathrm{CN}_{3}$ at $70{ }^{\circ} \mathrm{C}$ for $10 \mathrm{~min}$, and then additional $\left[\mathrm{Pd}\left(\mathrm{CH}_{3} \mathrm{CN}\right)_{4}\right]\left(\mathrm{BF}_{4}\right)_{2}\left(3 \mu \mathrm{mol}, 100 \mu \mathrm{L}\right.$ of a $30 \mathrm{~mm}$ stock solution in $\left.\mathrm{CD}_{3} \mathrm{CN}\right)$ was added into the mixture. After $50 \mathrm{~min}$ heating at the same temperature, ${ }^{1} \mathrm{H}$ NMR spectroscopy confirmed the quantitative formation of the cage compound.

1H-NMR $\left(300 \mathrm{MHz}, \mathrm{CD}_{3} \mathrm{CN}\right) \delta 2.98-3.11\left(16 \mathrm{H}, \mathrm{m}, \mathrm{CH}_{2}\right), 3.45\left(24 \mathrm{H}, \mathrm{s}, \mathrm{OCH}_{3}\right), 3.70-3.83(16 \mathrm{H}$, $\left.\mathrm{m}, \mathrm{CH}_{2}\right), 7.25\left(16 \mathrm{H}, \mathrm{d},{ }^{3} \mathrm{~J}=7.8 \mathrm{~Hz}, \mathrm{H}_{\mathrm{b}}\right), 7.63\left(16 \mathrm{H}, \mathrm{dd},{ }^{3} \mathrm{~J}=7.8 \mathrm{~Hz},{ }^{4} \mathrm{~J}=1.8 \mathrm{~Hz}, \mathrm{H}_{\mathrm{c}}\right), 7.98(32 \mathrm{H}$, $\left.\mathrm{dd},{ }^{3} J=6.9 \mathrm{~Hz},{ }^{4} J=1.2 \mathrm{~Hz}, \mathrm{H}_{\mathrm{d}}\right), 8.16\left(16 \mathrm{H}, \mathrm{dd},{ }^{3} J=6.9 \mathrm{~Hz},{ }^{4} J=1,5 \mathrm{~Hz}, \mathrm{H}_{\mathrm{f}}\right), 8.28\left(16 \mathrm{H}, \mathrm{d},{ }^{4} J=\right.$ $\left.1.8 \mathrm{~Hz}, \mathrm{H}_{\mathrm{a}}\right), 8.99\left(32 \mathrm{H}, \mathrm{dd},{ }^{3} J=6.9 \mathrm{~Hz},{ }^{4} J=1.2 \mathrm{~Hz}, \mathrm{H}_{\mathrm{e}}\right), 9.07\left(16 \mathrm{H}, \mathrm{dd},{ }^{3} J=6.9 \mathrm{~Hz},{ }^{4} J=1.5 \mathrm{~Hz}\right.$, $\mathrm{H}_{\mathrm{g}}$. 


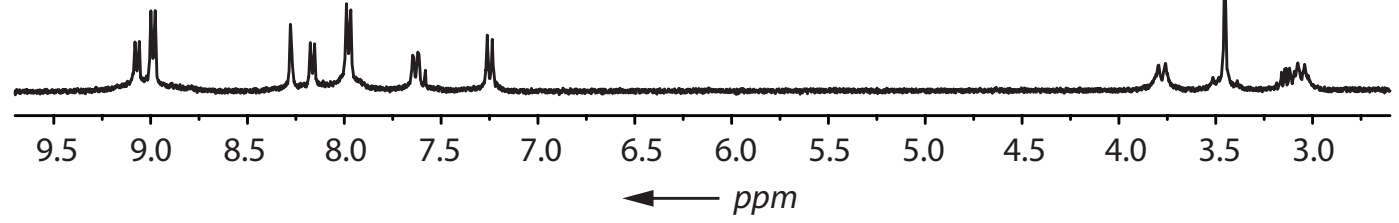

${ }^{13}$ C-NMR (125 MHz, CD $\left.{ }_{3} \mathrm{CN}\right) \delta 31.33,53.84,86.04,88.97,96.62,124.94,125.48,128.71$, $130.98,133.20,134.54,136.08,141.26,141.45,151.49,151.98,152.18$.

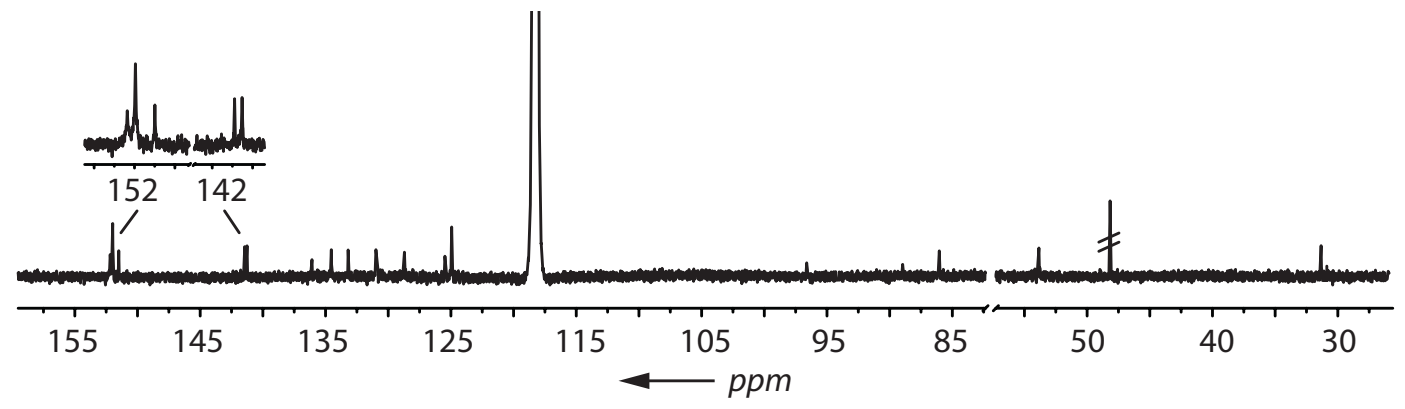

HRMS (ESI, pos.) $m / z\left(\left[\mathrm{Pd}_{6} \mathbf{L}^{2}{ }_{8}+7 \mathrm{BF}_{4}\right]^{5+}\right)$ calcd. $=1016.6109$, found $=1016.6119$.

As described in the cage synthesis of $\operatorname{Pd}_{6} \mathbf{L}_{8}{ }^{2}$, the ligand $\mathbf{L}^{2}$ was treated with 0.5 equivalents of $\mathrm{Pd}$ II and heated at $70{ }^{\circ} \mathrm{C}$ for 10 min to give intermediate $\operatorname{Pd}_{4} \mathbf{L}_{8}{ }_{8}$, and the solution was immediately subjected to NMR spectroscopic measurements (Figure 4-7 and Figure 4-11). Then, a further 0.25 equivalents of $\mathrm{Pd}^{\mathrm{II}}$ was added to the solution of $\operatorname{Pd}_{4} \mathbf{L}^{2}{ }_{8}$ and heated for another $50 \mathrm{~min}$. 1D and 2D ${ }^{1} \mathrm{H}$ NMR spectra demonstrated the complete formation of $\operatorname{Pd}_{6} \mathbf{L}_{8}$ (Figure 4-8 and Figure 4-12).

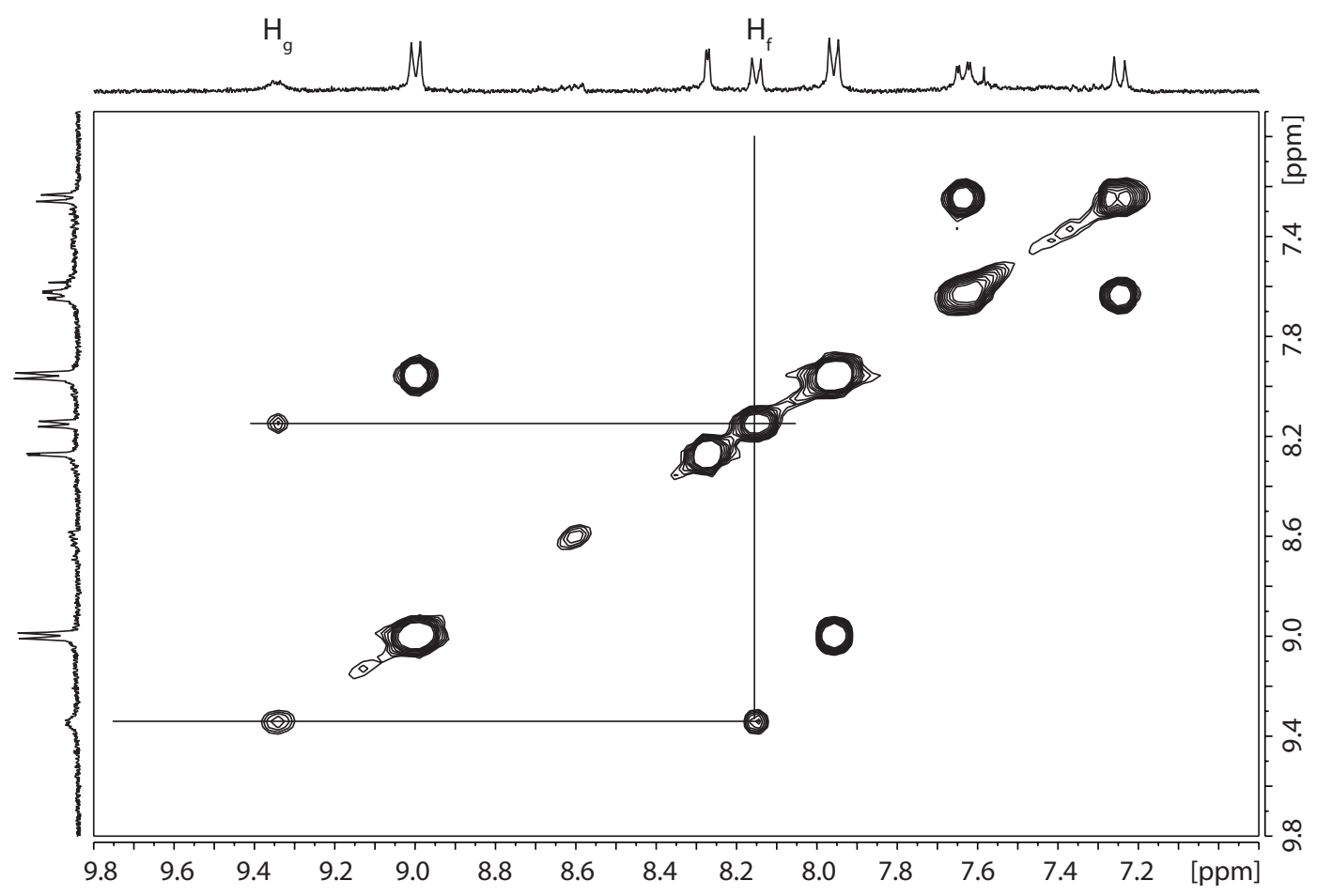

Figure 4-11 The ${ }^{1} \mathrm{H}-{ }^{1} \mathrm{H}$ COSY spectrum of the intermediate $\mathrm{Pd}_{4} \mathrm{~L}_{8}^{2}$ in $\mathrm{CD}_{3} \mathrm{CN}$. 


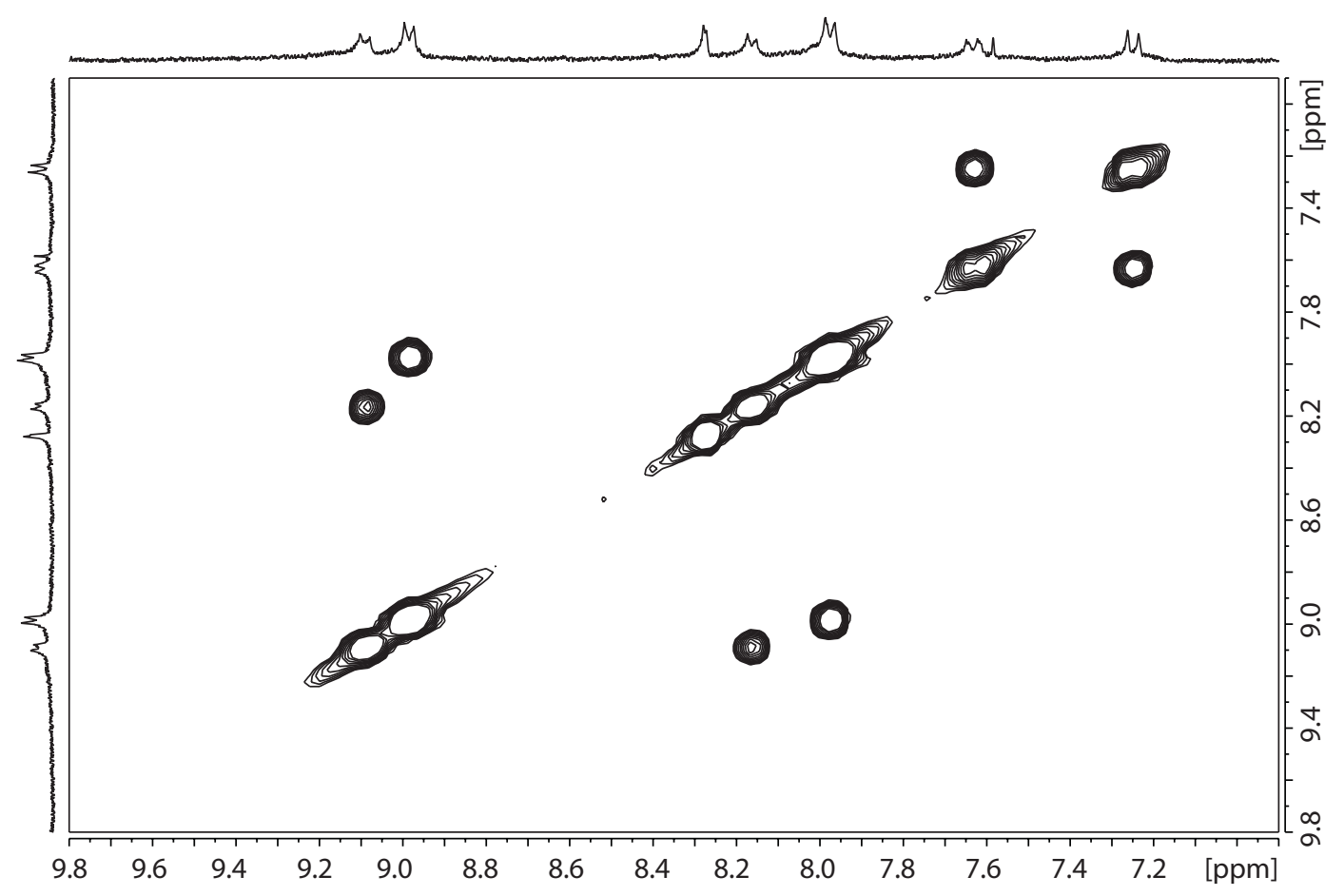

Figure 4-12 The ${ }^{1} \mathrm{H}-{ }^{-1} \mathrm{H}$ COSY spectrum of $\mathrm{Pd}_{6} \mathbf{L}^{2}{ }_{8}$ obtained by adding 0.25 equiv. of $\mathrm{Pd}^{\text {II }}$ into $\mathrm{Pd}_{4} \mathbf{L}_{8}{ }_{8}$ in $\mathrm{CD}_{3} \mathrm{CN}$.

\subsubsection{DOSY comparison of both cages}

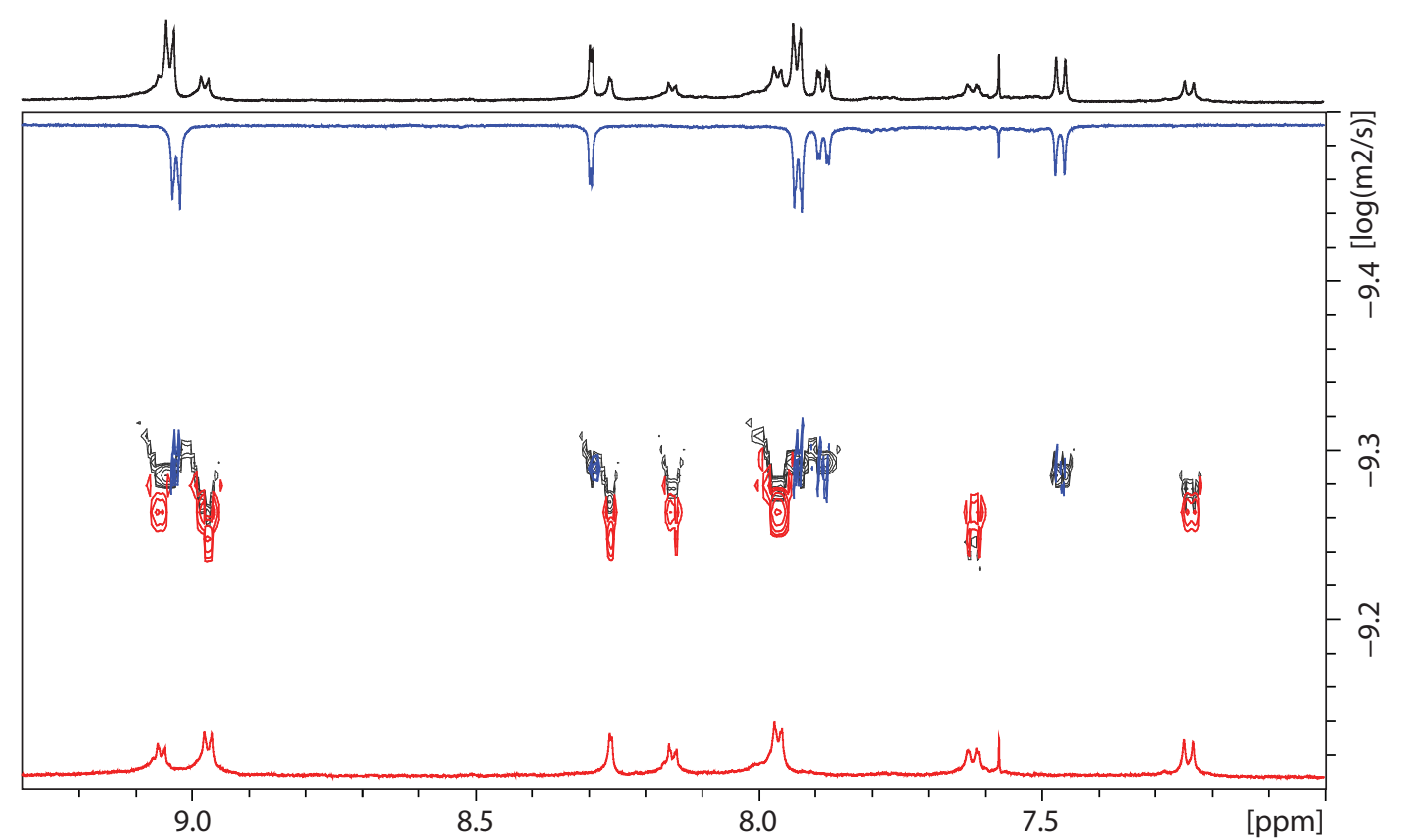

Figure 4-13 Superposition of DOSY spectra (500 MHz, $\mathrm{CD}_{3} \mathrm{CN}, 298 \mathrm{~K}$ ) of cage complexes $\operatorname{Pd}_{6} \mathbf{L}_{12}$ (blue), $\operatorname{Pd}_{6} \mathbf{L}_{8}$ (red), and the 1:1 mixture of both cages (black). 
The hydrodynamic radii can be calculated from the diffusion coefficients using the StokesEinstein equation:

$$
r=\frac{k \cdot T}{6 \cdot \pi \cdot \eta \cdot D}
$$

With $r$ = radius, $k=$ Boltzmann const., $T=$ temperature, $\eta=$ dynamic viscosity of $\mathrm{CD}_{3} \mathrm{CN}$ $\left(3.69 \times 10^{-4} \mathrm{~Pa} / \mathrm{s}\right)^{[55]}$ and $D=$ diffusion coefficients estimated by the DOSY experiment.

Table 4-2 Calculated hydrodynamic radii for cage complexes and host-guest complexes.

\begin{tabular}{|c|c|c|}
\hline complex & $\operatorname{Pd}_{6} \mathbf{L}_{12}$ & $\operatorname{Pd}_{6} \mathbf{L}^{2}$ \\
\hline $\log D$ & -9.29 & -9.26 \\
\hline$D\left[\times 10^{-10} \mathrm{~m}^{2} \mathrm{~s}^{-1}\right]$ & 5.17 & 5.51 \\
\hline$r[\AA]$ & 11.5 & 10.8 \\
\hline
\end{tabular}




\subsubsection{X-ray data of ligand $\mathbf{L}^{2}$}

The X-ray structure was measured, solved and refined by Reent Michel.

Table 4-3 Crystal data and structure refinement of ligand $\mathbf{L}^{2}$.

\begin{tabular}{|c|c|}
\hline Empirical formula & $\mathrm{C}_{33} \mathrm{H}_{25} \mathrm{~N}_{3} \mathrm{O}$ \\
\hline Formula Weight [gmol-1] & 479.56 \\
\hline Temperature $[\mathrm{K}]$ & $100(2)$ \\
\hline Wavelength $[\AA ̊]$ & 0.71073 \\
\hline Crystal system & Triclinic \\
\hline Space group & $P \overline{\mathbf{1}}$ \\
\hline Unit cell dimension & $\begin{array}{ll}\mathrm{a}=10.424(2) \AA & \alpha=65.60(2)^{\circ} \\
\mathrm{b}=11.196(2) \AA & \beta=65.60(2)^{\circ} \\
\mathrm{c}=12.161(2) \AA & \gamma=65.60(2)^{\circ}\end{array}$ \\
\hline Volume $\left[\AA^{3}\right]$ & $1222.1(5)$ \\
\hline $\mathrm{Z}$ & 2 \\
\hline$r_{\text {calc }}\left[\mathrm{Mg} / \mathrm{m}^{3}\right]$ & 1.303 \\
\hline$\sigma\left[\mathrm{mm}^{-1}\right]$ & 0.080 \\
\hline$F(000)$ & 504 \\
\hline Crystal size $\left[\mathrm{mm}^{3}\right]$ & $0.1 \times 0.1 \times 0.1$ \\
\hline$\theta_{\min } / \theta_{\max }$ & $1.929 / 27.874^{\circ}$ \\
\hline Index ranges & $\begin{array}{l}-13 \leq h \leq 13 \\
-14 \leq k \leq 13\end{array}$ \\
\hline Reflections collected & 26776 \\
\hline Independent reflections & $5807\left[R_{\text {int }}=3.38 \%\right]$ \\
\hline Completeness to $\theta=25.242^{\circ}$ & $99.9 \%$ \\
\hline Absorption correction & Semiempirical from equivalents \\
\hline Max. and min. transmission & 0.7456 and 0.6919 \\
\hline Refinement method & Full-matrix least-squares on $F^{2}$ \\
\hline Data / restraints / parameters & $5807 / 0 / 336$ \\
\hline GooF on $F^{2}$ & 1.051 \\
\hline Final $R$ indices $[I>2 \sigma(I)]$ & $R_{1}=4.47 \%, w R_{2}=10.32 \%$ \\
\hline$R$ indices (all data) & $R_{1}=6.11 \%, w R_{2}=11.29 \%$ \\
\hline Extinction coefficient & $0.0045(13)$ \\
\hline Largest diff. peak and hole $\left[\mathrm{e} \AA^{-3}\right]$ & 0.334 and -0.235 \\
\hline
\end{tabular}


The single crystal was mounted in an inert oil.[56,57] The X-ray data set was collected at 100(2) K on a Bruker APEX II Quazar diffractometer, equipped with an INCOATEC Microfocus source (ImS) for mirror-monochromated MoK $\alpha$ radiation $(\lambda=0.71073 \AA$ ). Data were processed using Smart Apex II CCD detector from Bruker, and a multiscan absorption correction was applied using SADABS.[58]

The structure was solved using direct methods with SHELXT (G. Sheldrick, Göttingen 2012) and refined by full-matrix least-squares on $F^{2}$ (the squared structure factor), using SHELXL-2013/4[59-62] program. Non-hydrogen atoms were refined with anisotropic displacement parameters. Hydrogen atoms were included in calculated positions and refined using a riding-model. 


\subsubsection{Host-guest studies}

In the following table, an overview of titrated guest molecules is listed. None of the guest molecules have showed any interactions with the cage $\left[\mathrm{Pd}_{6} \mathbf{L}_{8}\right]\left(\mathrm{BF}_{4}\right)_{12}$ by $\mathrm{NMR}$ spectroscopy or ESI mass spectrometry.

Table 4-4 List of investigated guest molecules.

\begin{tabular}{|c|c|}
\hline Guests & Guests \\
\hline 4'-Hydroxyacetaanilide & Cobalt(II) tetraphenylporphyrin \\
\hline$(\mathrm{S})-(+)$-Clopidogrel sulfate & $\begin{array}{l}\text { Cobalt(III) biscyano } \\
\text { tetraphenylporphyrin (TBA salt) }\end{array}$ \\
\hline Carboplatin & $\begin{array}{l}\text { Tetraphenylporphyrin } \\
\text { tetrasulfonate (TBA salt) }\end{array}$ \\
\hline Caffeine & Guanine \\
\hline Sodium N-Cyclohexylsulfamate & Guanosine \\
\hline$o$-Benzoic sulfimide & $\begin{array}{c}\text { Guanosine Monophosphate } \\
\text { (disodium salt) }\end{array}$ \\
\hline Nickel Dimethylglyoxime & $\left(\mathrm{NBu}_{4}\right)_{2} \mathrm{~B}_{12} \mathrm{~F}_{12}$ \\
\hline D-(+)-Glucose & $\mathrm{C}_{60}$ \\
\hline Ferrocene & $\mathrm{K}_{4}\left[\mathrm{Au}_{4} \mathrm{O}_{4}\left(\mathrm{SeO}_{3}\right)_{4}\right] \cdot 6 \mathrm{H}_{2} \mathrm{O}$ \\
\hline$\left(\mathrm{NBu}_{4}\right)_{2} \mathrm{Mo}_{6} \mathrm{O}_{19}$ & $\mathrm{Na}_{6}\left[\mathrm{Pd}_{13}(\mathrm{AsPh})_{8} \mathrm{O}_{32}\right]_{3} \cdot 17 \mathrm{H}_{2} \mathrm{O}$ \\
\hline Tetraphenylporphyrin & $\mathrm{Na}_{8}\left[\mathrm{Pd}_{13} \mathrm{As}_{8} \mathrm{O}_{34}(\mathrm{OH})_{6}\right] \cdot 42 \mathrm{H}_{2} \mathrm{O}$ \\
\hline
\end{tabular}




\subsubsection{Molecular modelling results}

\subsubsection{PM6 modelling of cubic coordination cage $\operatorname{Pd}_{6} \mathbf{L}^{\mathbf{1}}{ }_{12}$}

The structure of cage $\operatorname{Pd}_{6} \mathbf{L}_{12}$ was optimized on the semiempiric PM6 level of theory.[64] The result is shown in Figure 4-14. All faces constructed by Pd(pyridine) ${ }_{4}$ are equal and are marked with purple, dibenzosuberone backbones are in gray, oxygen atoms in red.

a)

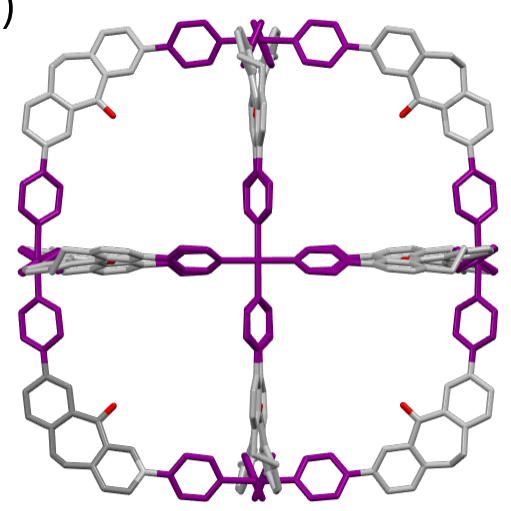

C)

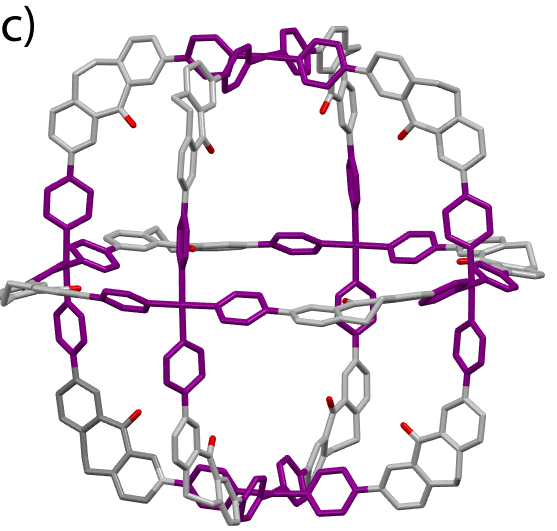

b)

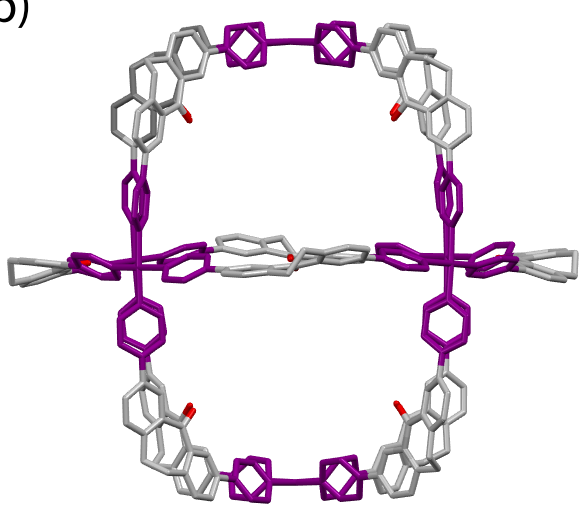

d)

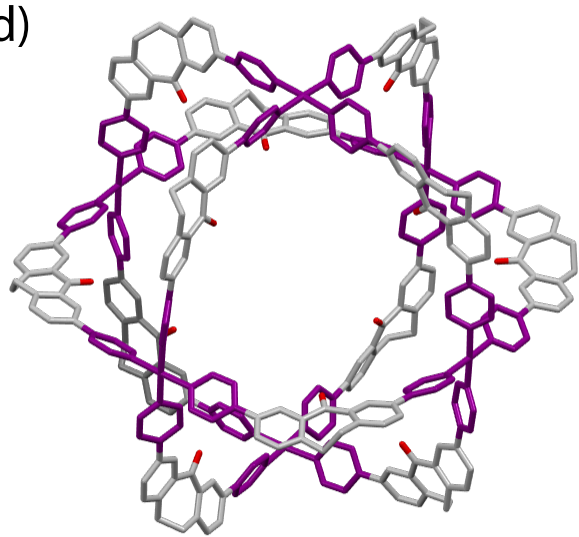

Figure 4-14 Different views of the PM6 structure of cubic coordination cage $\left[\operatorname{Pd}_{6} \mathbf{L}^{\mathbf{1}_{12}}\right]^{12+}$.

\subsubsection{Data extracted from the ligands Backbone ${ }_{1-6} X-a_{\text {arm }} \mathrm{A} Y$-arma-c modelling}

All 54 possible ligand structures with the form Backbone 1-6 $_{-6} X-\operatorname{arm}_{\mathrm{A}-\mathrm{c}} Y$-arma-c, which are chemically plausible and synthetically relative easy to achieve, were geometry optimized at DFT B3LYP/6-31G(d,p) level of theory. The arm-length values $x, y$ and the angle $\varphi, \theta$ were extracted from the models. Lengths are given in angstroms $(\AA)$, and angles are measured in degrees. The $x / y$ and $\varphi / \theta$ combinations were inserted into the plotting graph of the function $\varphi / \theta=\mathrm{f}(x / y)$. 


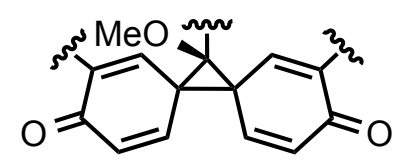

Table 4-5 Values of arm-lengths and angles extracted from model of $\mathbf{1} X$-arm $\mathrm{A-c}$ Y-arma-c.

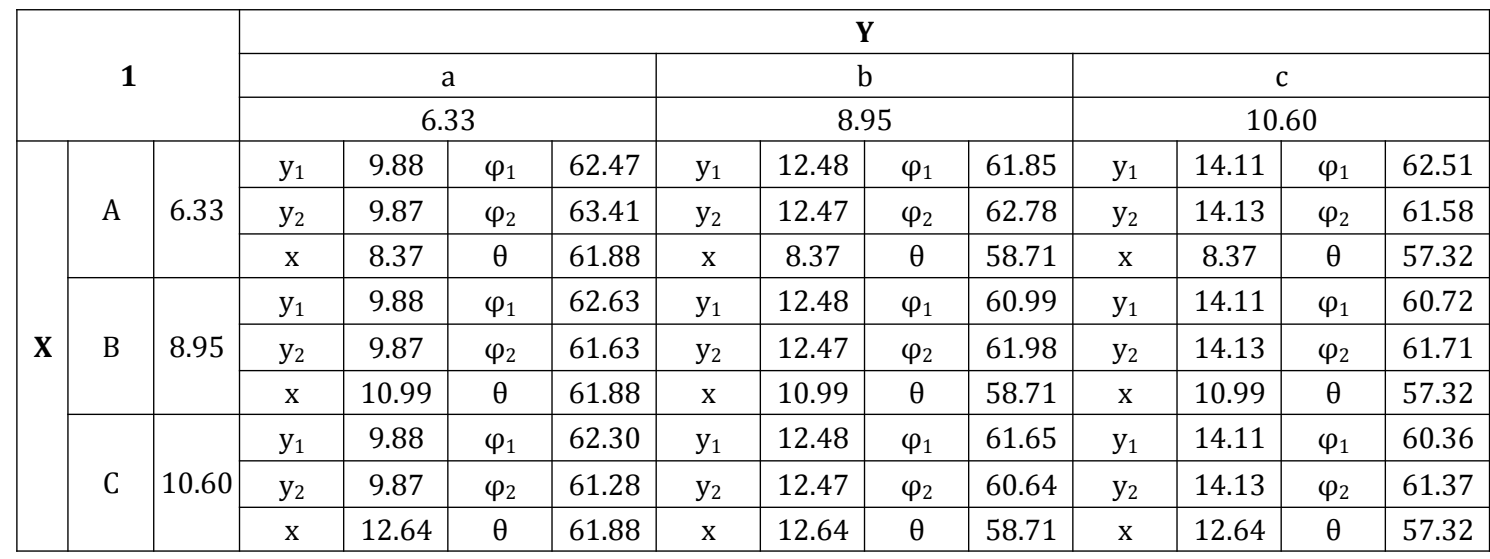

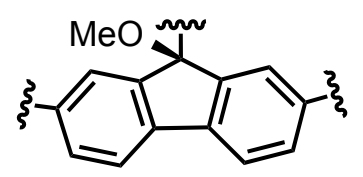

Table 4-6 Values of arm-lengths and angles extracted from model of $2 X$ - $\operatorname{arm}_{\mathrm{A}-\mathrm{c}} Y$-arma-c.

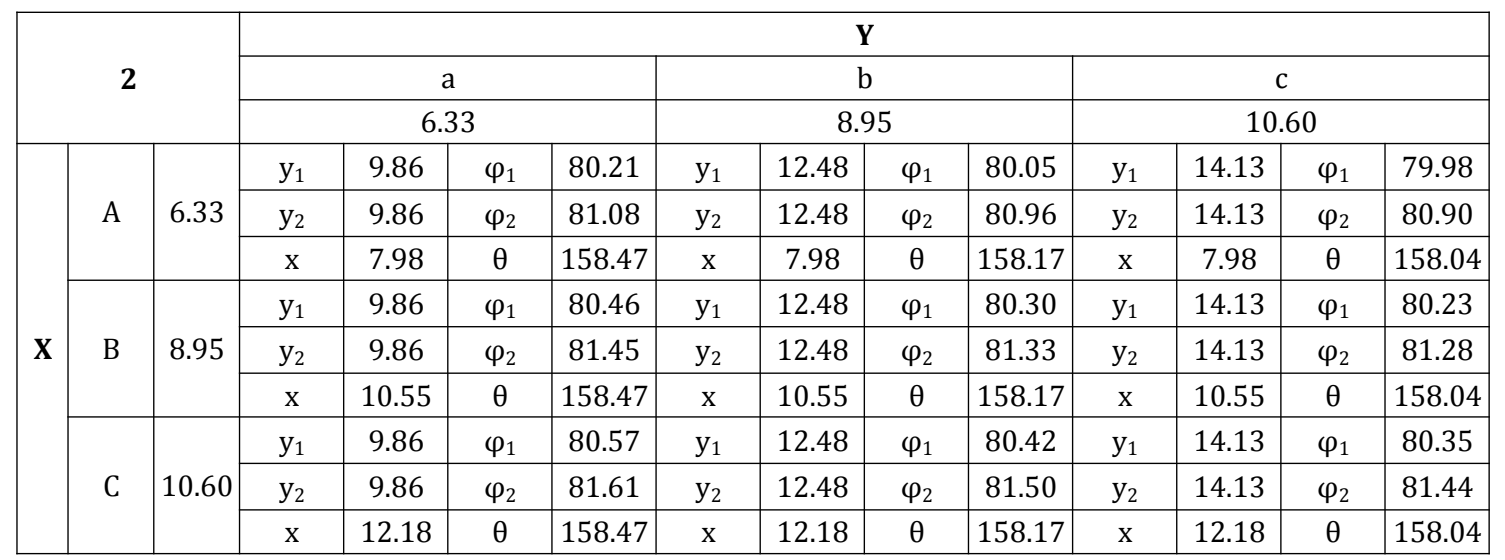




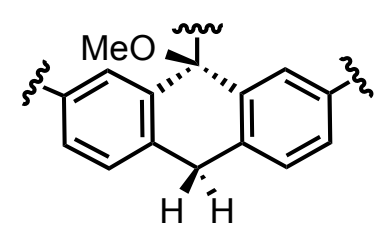

Table 4-7 Values of arm-lengths and angles extracted from model of $3 X$-arm $\mathrm{A-c} Y$ - $\operatorname{arm}_{\mathrm{a}-\mathrm{c}}$.

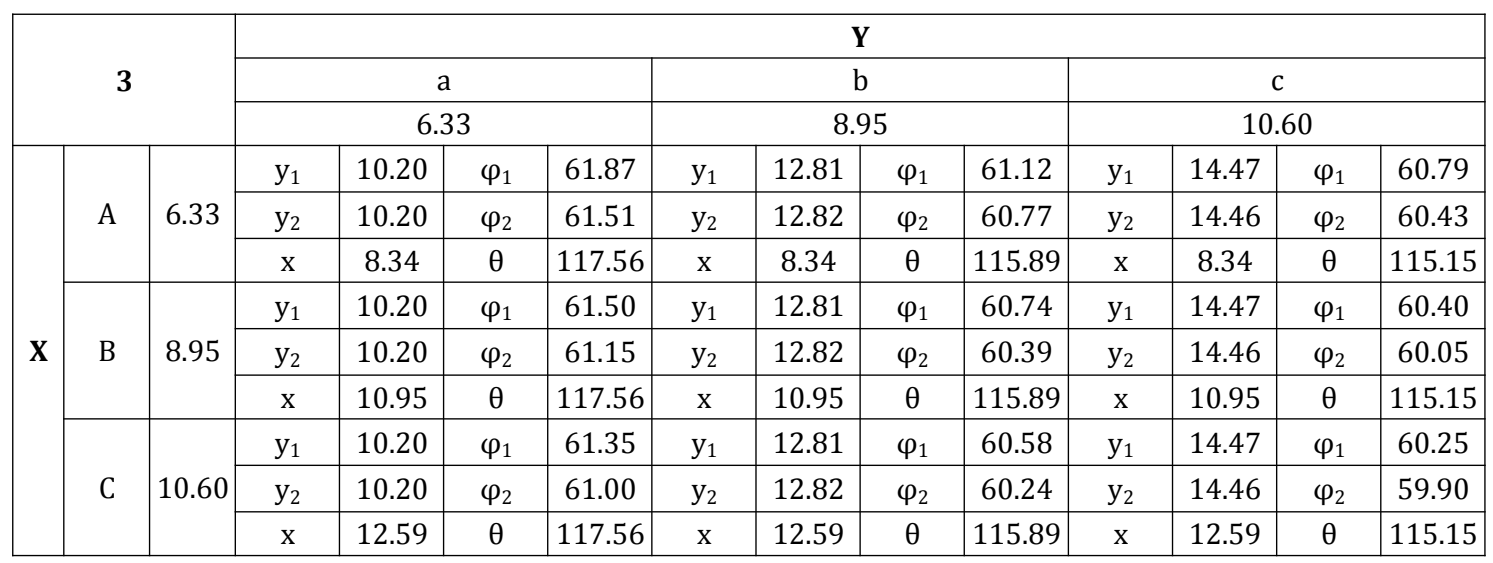

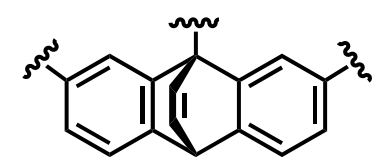

Table 4-8 Values of arm-lengths and angles extracted from model of $\mathbf{4} X-\operatorname{arm}_{\mathrm{A}-\mathrm{c}} Y$ - $\operatorname{arm}_{\mathrm{a}-\mathrm{c}}$.

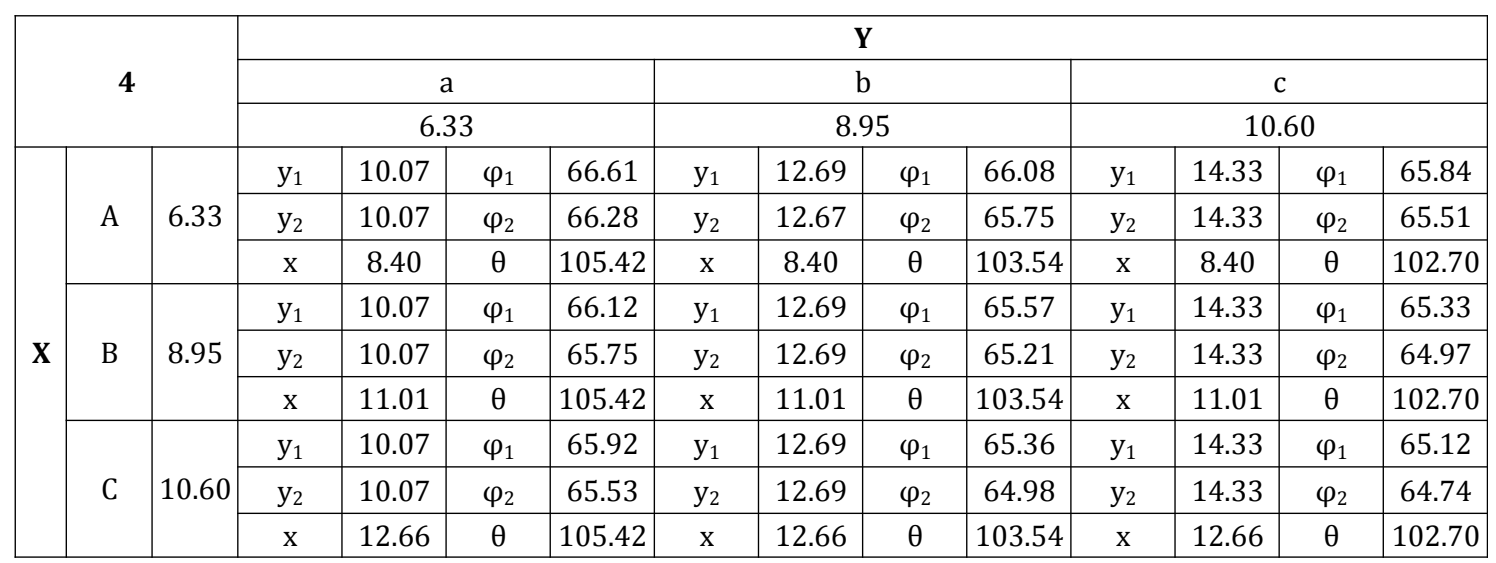




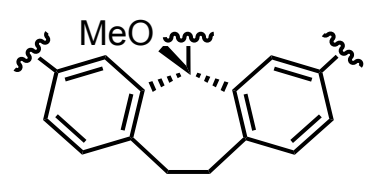

Table 4-9 Values of arm-lengths and angles extracted from model of $5 X$-arm $\mathrm{A-c} Y$-arma-c.

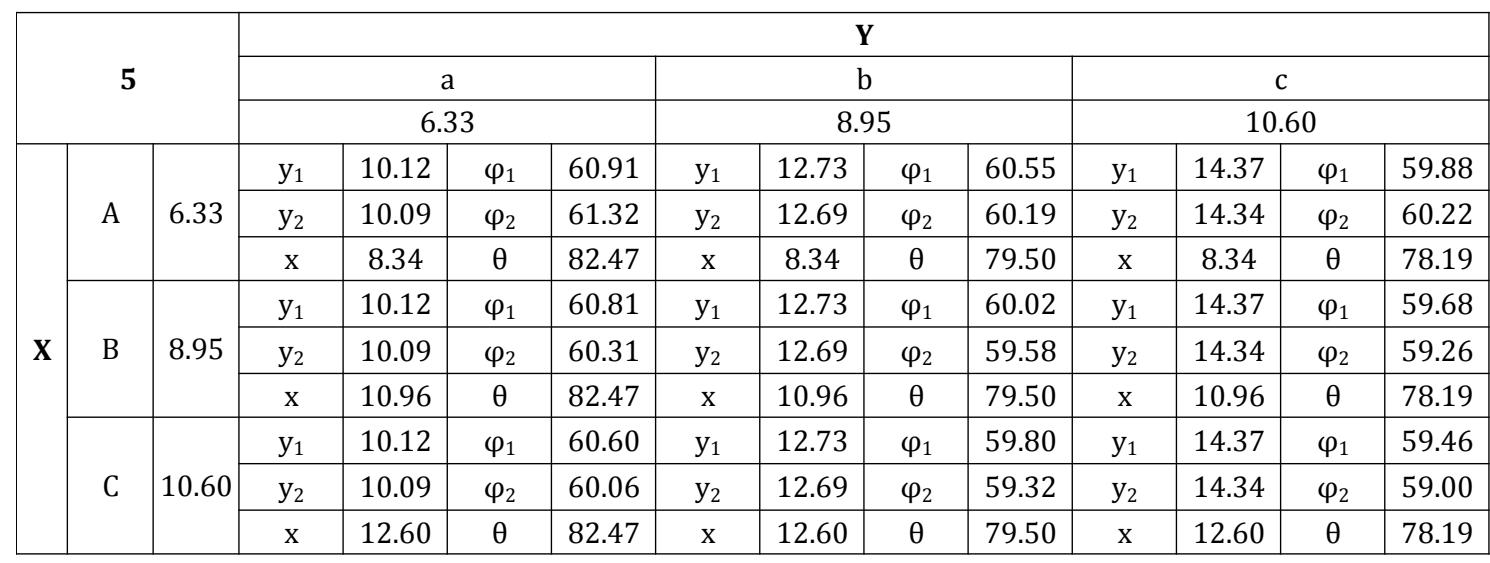

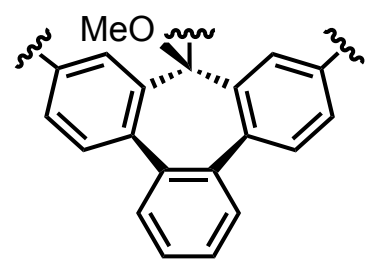

Table 4-10 Values of arm-lengths and angles extracted from model of $6 \mathrm{X}$ - $\operatorname{arm}_{\mathrm{A}-\mathrm{c}} Y$-arma-c.

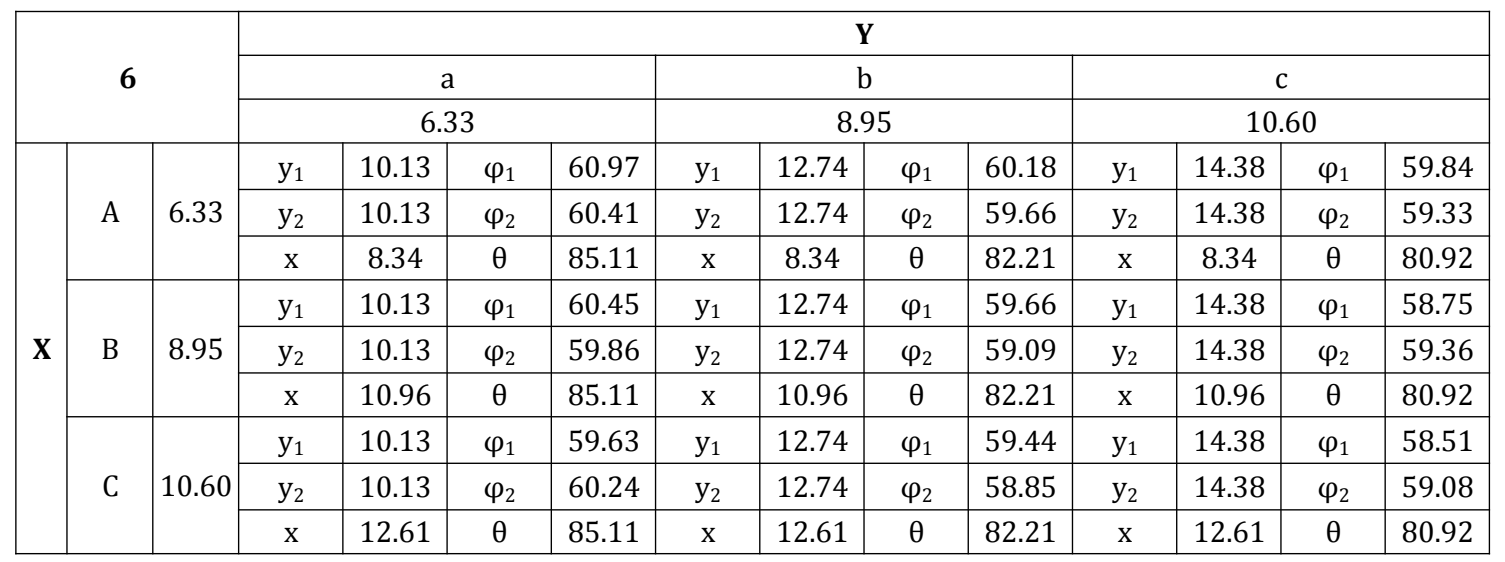




\subsection{References}

[1] M. M. J. Smulders, I. A. Riddell, C. Browne, J. R. Nitschke, Chem. Soc. Rev. 2012, 42, 1728.

[2] J. W. Steed, J. L. Atwood, Supramolecular Chemistry, WILEY, 2nd Ed. 2009.

[3] M. D. Ward, P. R. Raithby, Chem. Soc. Rev. 2013, 42, 1619.

[4] R. S. Forgan, J.-P. Sauvage, J. F. Stoddart, Chem. Rev. 2011, 111, 5434.

[5] J. E. Beves, B. A. Blight, C. J. Campbell, D. A. Leigh, R. T. McBurney, Angew. Chem. Int. Ed. 2011, 50, 9260.

[6] D. M. Engelhard, S. Freye, K. Grohe, M. John, G. H. Clever, Angew. Chem. Int. Ed. 2012, $51,4747$.

[7] C.-F. Lee, D. A. Leigh, R. G. Pritchard, D. Schultz, S. J. Teat, G. A. Timco, R. E. P. Winpenny, Nature, 2009, 458, 314.

[8] G. H. Clever, M. Shionoya, Chem. Eur. J. 2010, 16, 11792.

[9] M. D. Pluth, K. N. Raymond, Chem. Soc. Rev. 2007, 36, 161.

[10] S. J. Dalgarno, N. P. Power, J. L. Atwood, Coord. Chem. Rev. 2008, 252, 825.

[11] D. J. Tranchemontagne, Z. Ni, M. O’Keeffe, O. M. Yaghi, Angew. Chem. Int. Ed. 2008, 47, 5136.

[12] R. Chakrabarty, P. S. Mukherjee, P. J. Stang, Chem. Rev. 2011, 111, 6810.

[13] T. K. Ronson, S. Zarra, S. P. Black, J. R. Nitschke, Chem. Commun. 2013, 49, 2476.

[14] S. Kubik, Top. Curr. Chem. 2012, 319, 1.

[15] T. Nakamura, H. Ube, M. Shionoya, Chem. Lett. 2013, 42, 328.

[16] S. Torquato, Y. Jiao, Nature, 2009, 460, 876.

[17] Y.-R. Zheng, W.-J. Lan, M. Wang, T. R. Cook, P. J. Stang, J. Am. Chem. Soc. 2011, 133, 17045.

[18] X. Sun, D. W. Johnson, D. L. Caulder, R. E. Powers, K. N. Raymond, E. H. Wong, Angew. Chem. Int. Ed. 1999, 38, 1303.

[19] S. Hiraoka, Y. Sakata, M. Shionoya, J. Am. Chem. Soc. 2008, 130, 10058.

[20] M. Wang, V. Vajpayee, S. Shanmugaraju, Y.-R. Zheng, Z. Zhao, H. Kim, P. S. Mukherjee, K.-W. Chi, P. J. Stang, Inorg. Chem. 2011, 50, 1506.

[21] M. Yoshizawa, M. Nagao, K. Kumazawa, M. Fujita, J. Organomet. Chem. 2005, 690, 5383.

[22] P. N. W. Baxter, J.-M. Lehn, G. Baum, D. Fenske, Chem. Eur. J. 1999, 5, 102.

[23] B. Therrien, G. Süss-Fink, P. Govindaswamy, A. K. Renfrew, P. J. Dyson, Angew. Chem. Int. Ed. 2008, 47, 3773.

[24] P. J. Lusby, P. Müller, S. J. Pike, A. M. Z. Slawin, J. Am. Chem. Soc. 2009, 131, 16398.

[25] Y.-R. Zheng, Z. Zhao, M. Wang, K. Ghosh, J. B. Pollock, T. R. Cook, P. J. Stang, J. Am. Chem. Soc. 2010, 132, 16873.

[26] A. K. Bar, S. Mohapatra, E. Zangrando, P. S. Mukherjee, Chem. Eur. J. 2012, 18, 9571.

[27] S. Bivaud, J.-Y. Balandier, M. Chas, M. Allain, S. Goeb, M. Sallé, J. Am. Chem. Soc. 2012, $134,11968$. 
[28] S. Neogi, Y. Lorenz, M. Engeser, D. Samanta, M. Schmittel, Inorg. Chem. 2013, 52, 6975.

[29] F. Schmitt, J. Freudenreich, N. P. E. Barry, L. Juillerat-Jeanneret, G. Süss-Fink, B. Therrien, J. Am. Chem. Soc. 2012, 134, 754.

[30] Y. Yamanoi, Y. Sakamoto, T. Kusukawa, M. Fujita, S. Sakamoto, K. Yamaguchi, J. Am. Chem. Soc. 2001, 123, 980.

[31] M. Aoyagi, K. Biradha, M. Fujita, J. Am. Chem. Soc. 1999, 121, 7457.

[32] M. Wang, Y.-R. Zheng, K. Ghosh, P. J. Stang, J. Am. Chem. Soc. 2010, 132, 6282.

[33] E. Alessio, M. Casanova, E. Zangrando, E. Iengo, Chem. Commun. 2012, 48, 5112.

[34] Q. F. Sun, J. Iwasa, D. Ogawa, Y. Ishido, S. Sato, T. Ozeki, Y. Sei, K. Yamaguchi, M. Fujita, Science 2010, 328, 1144.

[35] Q.-F. Sun, T. Murase, S. Sato, M. Fujita, Angew. Chem. Int. Ed. 2011, 50, 10318.

[36] Q.-F. Sun, S. Sato, M. Fujita, Nat. Chem. 2012, 4, 330.

[37] J.-R. Li, D. J. Timmons, H.-C. Zhou, J. Am. Chem. Soc. 2009, 131, 6368.

[38] J.-R. Li, A. A. Yakovenko, W. Lu, D. J. Timmons, W. Zhuang, D. Yuan, H.-C. Zhou, J. Am. Chem. Soc. 2010, 132, 17599.

[39] K. Suzuki, M. Tominaga, M. Kawano, M. Fujita, Chem. Commun. 2009, 1638.

For examples of edge- and vertex-centred cubic coordination cages see [40-43]:

[40] M. M. J. Smulders, A. Jiménez, J. R. Nitschke, Angew. Chem. Int. Ed. 2012, 51, 6681.

[41] C. Browne, S. Brenet, J. K. Clegg, J. R. Nitschke, Angew. Chem. Int. Ed. 2013, 52, 1944.

[42] W. Meng, B. Breiner, K. Rissanen, J. D. Thoburn, J. K. Clegg, J. R. Nitschke, Angew. Chem. Int. Ed. 2011, 50, 3479.

[43] S. C. Johannessen, R. G. Brisbois, J. P. Fischer, P. A. Grieco, A. E. Counterman, D. E. Clemmer, J. Am. Chem. Soc. 2001, 123, 3818.

[44] S. Freye, J. Hey, A. Torras Galán, D. Stalke, R. Herbst Irmer, M. John, G. H. Clever, Angew. Chem. Int. Ed. 2012, 51, 2191.

[45] S. Freye, D. M. Engelhard, M. John, G. H. Clever, Chem. Eur. J. 2013, 19, 2114.

[46] S. Freye, R. Michel, D. Stalke, M. Pawliczek, H. Frauendorf, G. H. Clever, J. Am. Chem. Soc. 2013, 135, 8476.

[47] M. Frank, J. Hey, I. Balcioglu, Y.-S. Chen, D. Stalke, T. Suenobu, S. Fukuzumi, H. Frauendorf, G. H. Clever, Angew. Chem. Int. Ed. 2013, 52, 10102.

[48] K. N. Houk, A. G. Leach, S. P. Kim, X. Zhang, Angew. Chem. Int. Ed. 2003, 42, 4872.

[49] M. Hong, Y. Zhao, W. Su, R. Cao, M. Fujita, Z. Zhou, A. S. C. Chan, J. Am. Chem. Soc. 2000, $122,4819$.

[50] D. Moon, S. Kang, J. Park, K. Lee, R. P. John, H. Won, G. H. Seong, Y. S. Kim, G. H. Kim, H. Rhee, M. S. Lah, J. Am. Chem. Soc. 2006, 128, 3530.

[51] T. K. Ronson, J. Fisher, L. P. Harding, M. J. Hardie, Angew. Chem, Int. Ed. 2007, 46, 9086.

[52] Which might find application in accommodating the NanoPutians: S. H. Chanteau J. M. Tour, J. Org. Chem. 2003, 68, 8750.

[53] B. T. Holmes, W. T. Pennington, T. W. Hanks, Syn. Comm. 2003, 33, 2447.

[54] Y. Wei, C-T. Chen, J. Am. Chem. Soc. 2007, 129, 7478. 
[55] David R. Lide, Ed., CRC Handbook of Chemistry and Physics, 89th Edition (Internet Version 2009), CRC Press/Taylor and Francis, Boca Raton, FL.

[56] T. Kottke, D. Stalke, J. Appl. Crystallogr. 1993, 26, 615-619.

[57] D. Stalke, Chem. Soc. Rev. 1998, 27, 171-178.

[58] G. M. Sheldrick, SADABS 20012/1, Göttingen, Germany, 2012.

[59] G. M. Sheldrick, SHELXT, Göttingen, Germany, 2012.

[60] G. M. Sheldrick, SHELXL-2013/4, Göttingen, Germany, 2013.

[61] G. M. Sheldrick, Acta Crystallogr., Sect. A. 2008, 64, 112.

[62] P. Müller, R. Herbst-Irmer, A. L. Spek, T. R. Schneider, M. R. Sawaya, Crystal Structure Refinement - A Crystallographer's Guide to SHELXL in IUCr Texts on Crystallography, Vol. 8 (Ed.: P. Müller), Oxford University Press, Oxford (England), 2006.

[63] A. L. Spek, Acta Crystallogr., Sect. D. 2009, D65, 148-155.

[64] Gaussian 09, M. J. Frisch, G. W. Trucks, H. B. Schlegel, G. E. Scuseria, M. A. Robb, J. R. Cheeseman, G. Scalmani, V. Barone, B. Mennucci, G. A. Petersson, H. Nakatsuji, M. Caricato, X. Li, H. P. Hratchian, A. F. Izmaylov, J. Bloino, G. Zheng, J. L. Sonnenberg, M. Hada, M. Ehara, K. Toyota, R. Fukuda, J. Hasegawa, M. Ishida, T. Nakajima, Y. Honda, O. Kitao, H. Nakai, T. Vreven, J. A. Montgomery, Jr., J. E. Peralta, F. Ogliaro, M. Bearpark, J. J. Heyd, E. Brothers, K. N. Kudin, V. N. Staroverov, R. Kobayashi, J. Normand, K. Raghavachari, A. Rendell, J. C. Burant, S. S. Iyengar, J. Tomasi, M. Cossi, N. Rega, J. M. Millam, M. Klene, J. E. Knox, J. B. Cross, V. Bakken, C. Adamo, J. Jaramillo, R. Gomperts, R. E. Stratmann, O. Yazyev, A. J. Austin, R. Cammi, C. Pomelli, J. W. Ochterski, R. L. Martin, K. Morokuma, V. G. Zakrzewski, G. A. Voth, P. Salvador, J. J. Dannenberg, S. Dapprich, A. D. Daniels, O. Farkas, J. B. Foresman, J. V. Ortiz, J. Cioslowski and D. J. Fox, Gaussian, Inc., Wallingford CT, 2009. 


\section{LIGHT-TRIGGERED GUEST UPTAKE AND RELEASE BY A PHOTOCHROMIC CAGE ${ }^{4}$}

In this chapter, a light-triggered photochromic coordination cage $\operatorname{Pd}_{2} \mathrm{~L}_{4}$ based on photoswitchable dithienylethene (DTE) units and square-planar-coordinated PdII ions is introduced. All four ligands exhibit reversible interconversion between a flexible "openring" form and a rigid "closed-ring" form under alternating irradiation wavelengths. This light-driven interconversion of the cages provides full dynamic and reversible control over the uptake and release of suitable guests, such as the spherical anion dodecafluorododecaborate $\left[\mathrm{B}_{12} \mathrm{~F}_{12}\right]^{2-}$.

\subsection{Introduction}

Diarylethene derivatives with heterocyclic aryl groups are well known as thermally irreversible photochromic compounds. The most important feature of these compounds is their high sensitivity and fatigue resistance. The reversible photochromic conversion between open- and closed-ring form of diarylethenes can be achieved by irradiation with

${ }^{4}$ This work presented in this chapter has been published: M. Han, R. Michel, B. He, Y.-S. Chen, D. Stalke, M. John, G. H. Clever, Angew. Chem. Int. Ed. 2013, 52, 1319. (“Hot Paper”) 
light of an appropriate wavelength (Figure 5-1).[1] Dithienylethene (DTE) derivatives are one of the most promising classes of compounds and have been widely applied.[2][3]

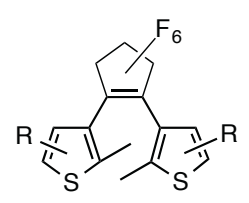

DTE-O

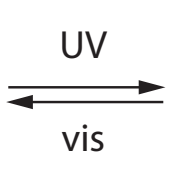

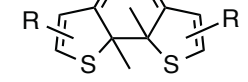

DTE-C

Figure 5-1 Photochromism of dithienylethene (DTE) derivatives.

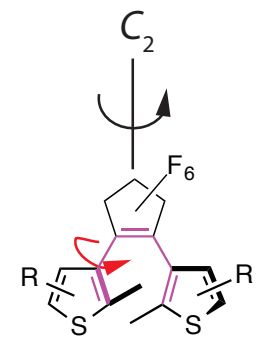

DTE-o

anti-parallel

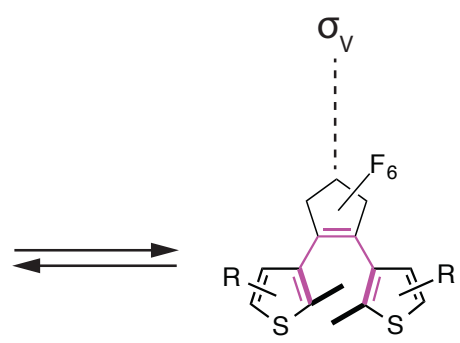

DTE-O

parallel

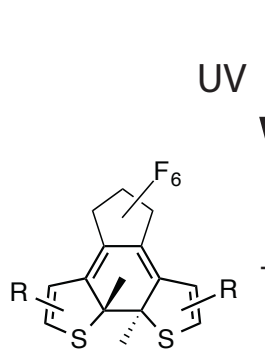

$(R, R)$

DTE-c

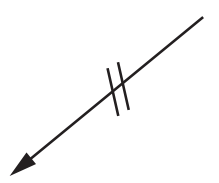

$\mathrm{R}$

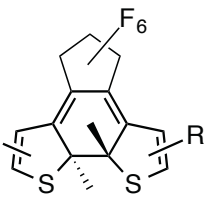

$(S, S)$

Figure 5-2 Conformations of dithienylethenes. The photoinactive parallel DTE-o possesses a mirror plane, and the photoactive anti-parallel DTE-o owns a $C_{2}$ axis across the molecule. It can form two enantiomers of DTE-c upon irradiation.

In the open-ring isomer, the molecule can rotate through the single $\mathrm{C}-\mathrm{C}$ bonds between the thiophene and cyclopenthene moieties. Therefore, the open-ring isomer adopts a bent conformation, and can exist in two major conformations: (i) the parallel conformation with the two thiophene rings in mirror symmetry and (ii) the anti-parallel conformation with a $C_{2}$ axis (Figure 5-2).[4] The photocyclization can only occur from the anti-parallel conformation upon UV irradiation, according to the Woodward-Hoffman rules.[5] The 1,3,5-hexatriene moiety (purple in Figure 5-2) can undergo both photo-chemically cyclization in the conrotatory mode and thermally in the disrotatory fashion. However, 
due to the steric hindrance of the methyl groups, the ring cyclization could not proceed thermally.

The closed-ring isomer DTE-c is nearly planar and exist as two enantiomers $(R, R)$ - and $(S, S)$ (Figure 5-2). While in the DTE-c isomer the $\pi$-electrons are delocalized through the whole molecule, in the DTE-c isomer the $\pi$-electrons are localized in the two thiophene rings, which results the differences in the physical properties. ${ }^{[6]}$

As discussed in Chapter 1, photoactive compounds, such as diarylethenes and azobenzenes can be used to introduce function into supramolecular host-guest systems. Discrete, molecular cages with guest encapsulating abilities, have been widely used in purification and separation, ${ }^{77,8]}$ the stabilization of reactive compounds, ${ }^{[9-17]}$ realization of sensing systems ${ }^{[18-22]}$, and capturing of hazardous chemicals.[23,24] For all these applications, a strong association between the host compound and guest molecules is required. Some systems require spatial and temporal control over the localization of the guest compounds either inside or outside of the host cavity to achieve dynamic control over the strength of the host-guest interaction. They were designed for the delivery, uptake and release of compounds [25-29], such as drugs or site-specific markers for bio-imaging. Furthermore, the dynamic control of the host-guest interaction could help to facilitate catalyst turnover and prevent the product inhibition,[30-35] which is one of the central limitations of supramolecular catalysis in confined cavities. Due to the significant advantages of light as an external stimulus, the interest grows steadily to develop light-responsive cage systems, ${ }^{[36-39]}$ which are able to control the uptake or ejection of guests in their cavities.

Light has been used as a trigger to reversibly control the encapsulation processes of specially designed guests[40-42] based on photoswitchable compounds.[43] Preparing the host compounds with a light-switchable component allows the application of lightresponsive guest uptake and release to a wide scope of guest species.[44] Starting with the pioneering work of Shinkai, ${ }^{[45]}$ Irie,[46] and Erlanger, ${ }^{[47]}$ a great deal of excellent work has been described. A large number of cyclodextrin-,[48] or calixarene-based[49] lightswitchable hosts, as well as supramolecular tweezers ${ }^{[50]}$ and ring compounds ${ }^{[51-53]}$ have been designed and studied. However, a light-switchable coordination cage system has not been introduced.[54]

In this chapter, a new metal-organic coordination cage[55-57] will be described, which was constructed with two square-planar-coordinated PdII ions and four photoswitchable bismonodentate dithienylethenyl (DTE) pyridyl ligands.[58-66] 

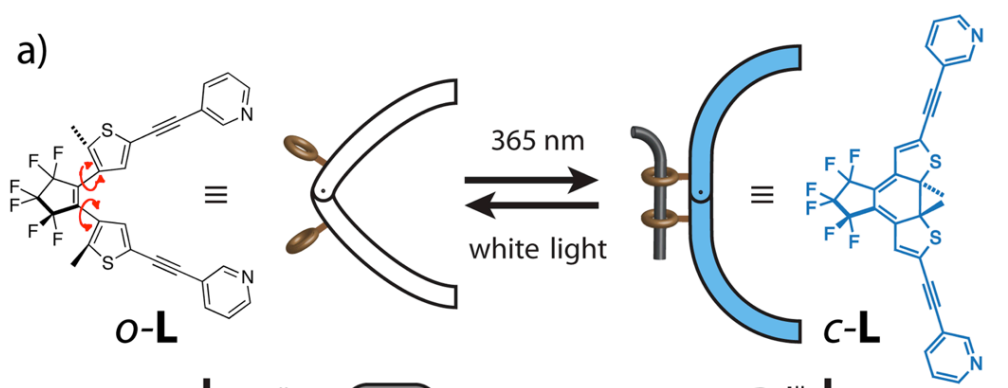

$\downarrow \mathrm{Pd}^{\prime \prime} \equiv \longrightarrow$

b)

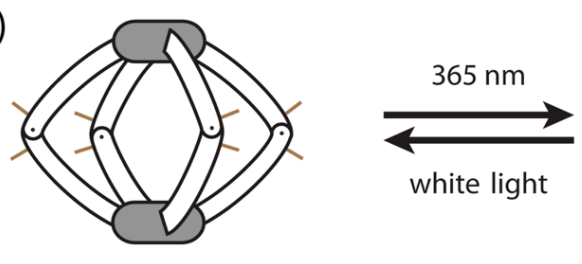

c)

O-C
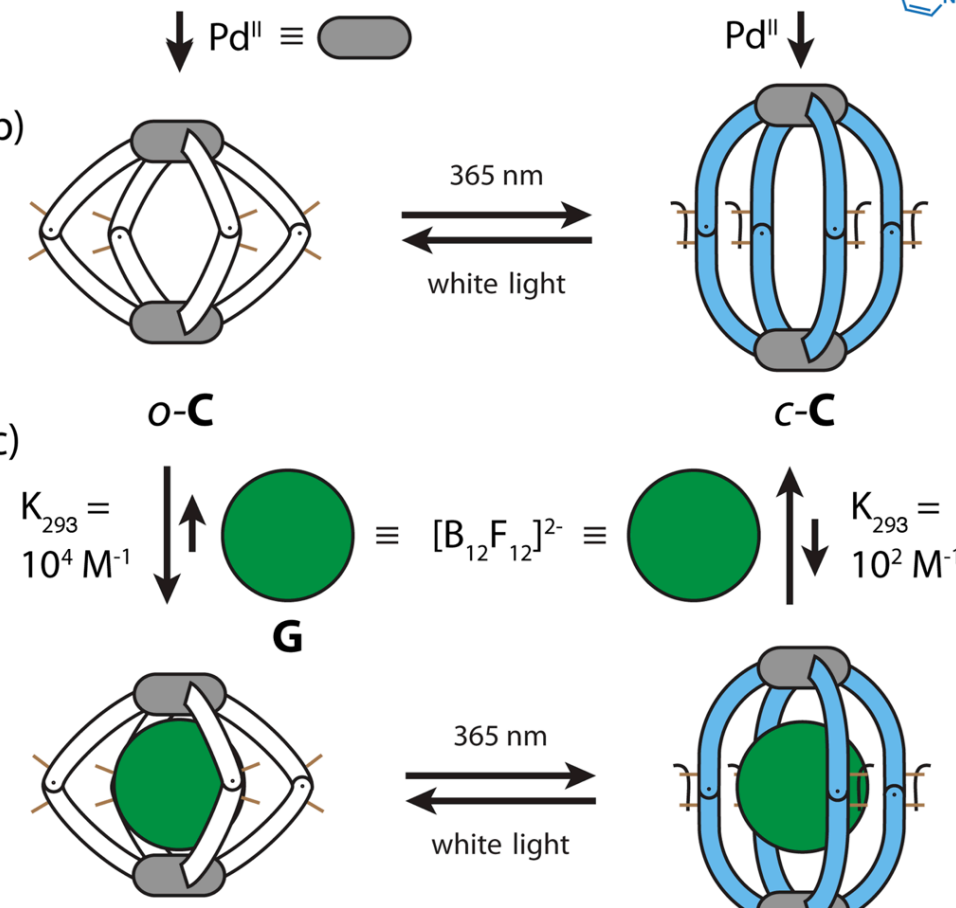

G@o-C
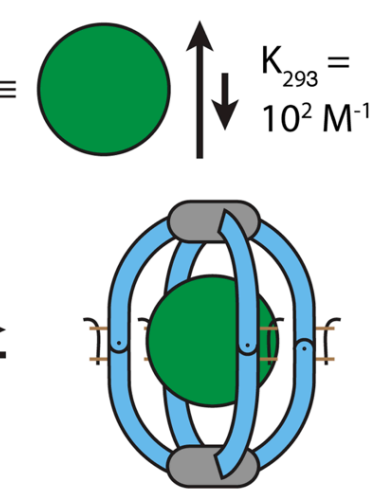

G@c-C

Figure 5-3 a) Upon irradiation at $365 \mathrm{~nm}$, the conformationally flexible dithienylethenyl pyridyl ligand $o$-L is converted into its rigid closed-ring isomer $c$-L. By irradiation with white light the process can be fully reversed back. b) Quantitative formation of both cages $o-\mathbf{C}=\left[\mathrm{Pd}_{2}(o-\mathbf{L})_{4}\right]\left(\mathrm{BF}_{4}\right)_{4}$ and $c-\mathbf{C}=\left[\mathrm{Pd}_{2}(c-\mathbf{L})_{4}\right]\left(\mathrm{BF}_{4}\right)_{4}$ by adding $\left[\mathrm{Pd}\left(\mathrm{CH}_{3} \mathrm{CN}\right)_{4}\right]\left(\mathrm{BF}_{4}\right)_{2}$. The above-mentioned photochemical processes can trigger the interconversion between two cage complexes as well. c) Spherical guest $\mathbf{G}=\left[\mathrm{B}_{12} \mathrm{~F}_{12}\right]^{2-}$ can be encapsulated in both cage isomers. Irradiation of the host-guest complexes results in the reversible uptake and release of the guest. Complex G@o-C is formed with much higher yield than $\mathbf{G} @ c-\mathbf{C}$ is formed from the rigid host $c$-C. Copyright (C) 2013 WILEY-VCH Verlag GmbH \& Co.

\subsection{Synthesis}

Ligand $o$-L was synthesized in a single step from perfluoro-1,2-bis(2-iodo-5-methylthien4-yl)cyclopentene ${ }^{[67,68]}$ and 3-ethinylpyridine by a Sonogashira cross-coupling procedure. The open-ring form ligand $(o-\mathbf{L})$, which is conformationally flexible, can be converted by irradiation with UV light $(365 \mathrm{~nm})$ to a rigid closed-ring form $(c-\mathbf{L})$, which could, in turn, 
be reconverted back to the open-ring form with white light (Figure 5-3a). The photocyclization of $o$-L in $\mathrm{CD}_{3} \mathrm{CN}$ could be followed by ${ }^{1} \mathrm{H}$ NMR spectroscopy, as a significant upfield shift $\left(\Delta \delta=-0.73 \mathrm{ppm}\right.$, Figure 5-4) of the thiophene proton signal $\mathrm{H}_{\mathrm{e}}$ was observed, as well as a less noticeable downfield shift ( $\Delta \delta=0.20 \mathrm{ppm}$, Figure 5-5) of the $\mathrm{H}_{\mathrm{f}}$ methyl singlet.

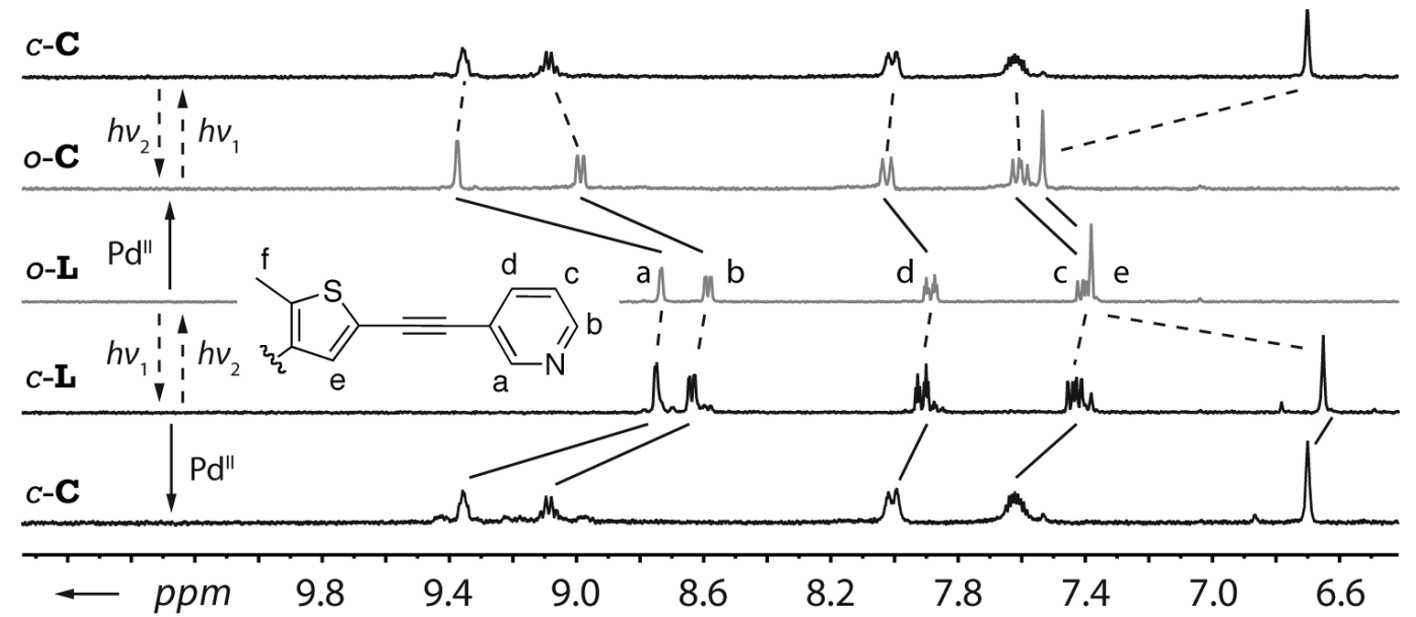

Figure 5-4 ${ }^{1} \mathrm{H}$ NMR spectra $\left(300 \mathrm{MHz}, \mathrm{CD}_{3} \mathrm{CN}\right.$ ) of ligands $o$-L, $c$-L and cages $o-\mathbf{C}, c$-C, aromatic region only. Copyright (C) 2013 WILEY-VCH Verlag GmbH \& Co.

When ligand $o$ - $\mathbf{L}$ or $c$ - $\mathbf{L}$ and $\left[\mathrm{Pd}\left(\mathrm{CH}_{3} \mathrm{CN}\right)_{4}\right]\left(\mathrm{BF}_{4}\right)_{2}$ at a $2: 1$ stoichiometry were heated in $\mathrm{CD}_{3} \mathrm{CN}$ at $70{ }^{\circ} \mathrm{C}$ for $1 \mathrm{~h}$, evidence for the formation of $\left[\mathrm{Pd}_{2}(o-\mathbf{L})_{4}\right]\left(\mathrm{BF}_{4}\right)_{4}(o-\mathbf{C})$ and $\left[\mathrm{Pd}_{2}(c-\mathbf{L})_{4}\right]\left(\mathrm{BF}_{4}\right)_{4}(c-\mathbf{C})$ was observed by the ${ }^{1} \mathrm{H}$ NMR spectroscopy (Figure 5-4 and Figure 5-5), in which both pyridine and thiophene signals shifted considerably downfield.

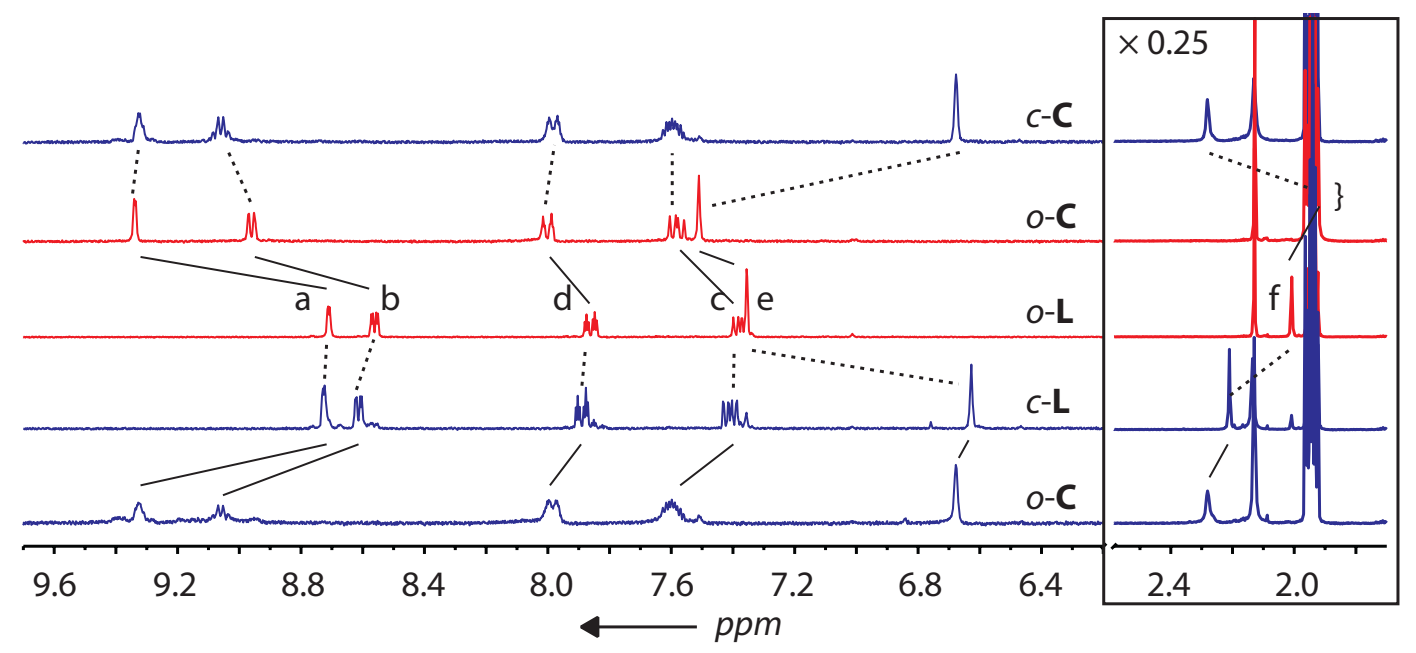

Figure 5-5 ${ }^{1} \mathrm{H}$ NMR spectra $\left(300 \mathrm{MHz}, \mathrm{CD}_{3} \mathrm{CN}\right)$ of $o$-L, $o$-C, $c$ - $\mathbf{L}$ and $c$-C including the aliphatic region. 


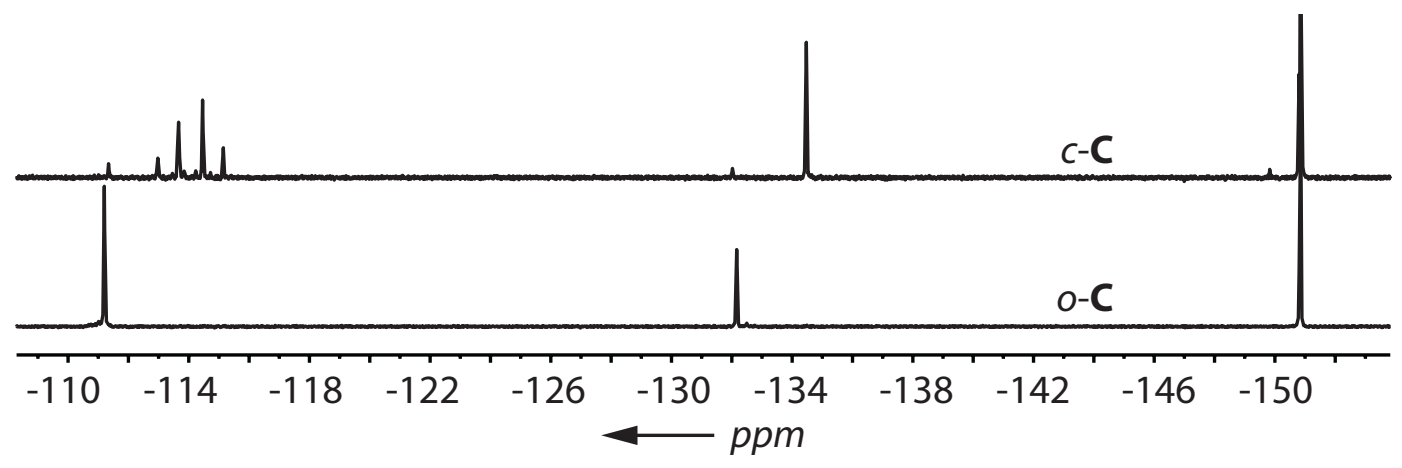

Figure 5-6 ${ }^{19} \mathrm{~F}$ NMR spectra $\left(376 \mathrm{MHz}, \mathrm{CD}_{3} \mathrm{CN}, 300 \mathrm{~K}\right)$ of $o-\mathrm{C}$ and $c$-C.

Similar to the photochemical behaviour of the free ligand, the cage compounds $o-\mathbf{C}$ and $c$-C can be also be interconverted reversibly by altering the wavelengths of the irradiation source. ${ }^{1 \mathrm{H}}$ and ${ }^{19} \mathrm{~F}$ NMR spectroscopy revealed evidence for the smooth isomerization processes between $\boldsymbol{o}-\mathbf{C}$ and $c$-C (Figure 5-4 and Figure 5-6). The ${ }^{19} \mathrm{~F}$ spectrum of $o-\mathbf{C}$ showed a peak at $\delta=-150.82 \mathrm{ppm}$ which was assigned to the $\mathrm{BF}_{4^{-}}$counter-anions, while two slightly broadened peaks (as a consequence of a fast exchange process) at $\delta=-132.14$ and $-111.17 \mathrm{ppm}$ in a 1:2 ratio were assigned to the fluorine atoms attached to the central and the two flanking $\mathrm{CF}_{2}$ groups per ligand. In contrast, the spectrum of the photoswitched closed-ring isomer $c$-C shows two doublets around $\delta=-114 \mathrm{ppm}$ for the flanking $\mathrm{CF}_{2}$ groups, which indicated an $\mathrm{AB}$-system in a non-exchanging, rigid system of a $C_{2}$-symmetric ligand clearly.

The UV-Vis absorption spectra were obtained to provide further evidence that structural interconversion is occurring in both the ligand and cage (Figure 5-7). Ligand $o$-L showed absorption maximum at $308 \mathrm{~nm}$, with a shoulder at $325 \mathrm{~nm}$, changes to the UV-Vis absorption spectrum of $o$-L were observed upon irradiation at $365 \mathrm{~nm}$. New absorbance appeared at $\lambda=240 \mathrm{~nm}$, and $594 \mathrm{~nm}$, with a decrease in absorbance at $325 \mathrm{~nm}$. Upon complexation of free ligand $o$-L with $\mathrm{Pd}$ II, the cage compound $o$-C gives two sharp absorption peaks at 256 and $314 \mathrm{~nm}$. Upon irradiation at $365 \mathrm{~nm}$ of $o$-C, the absorption at $256 \mathrm{~nm}$ disappeared, and the absorption of $c$ - $\mathbf{C}$ appeared as a shoulder band in the region between $369-440 \mathrm{~nm}$, together with a characteristic band for the ring-cyclization at 586 $\mathrm{nm}$. The intensity of the band at $314 \mathrm{~nm}$ was found to be decreased. 


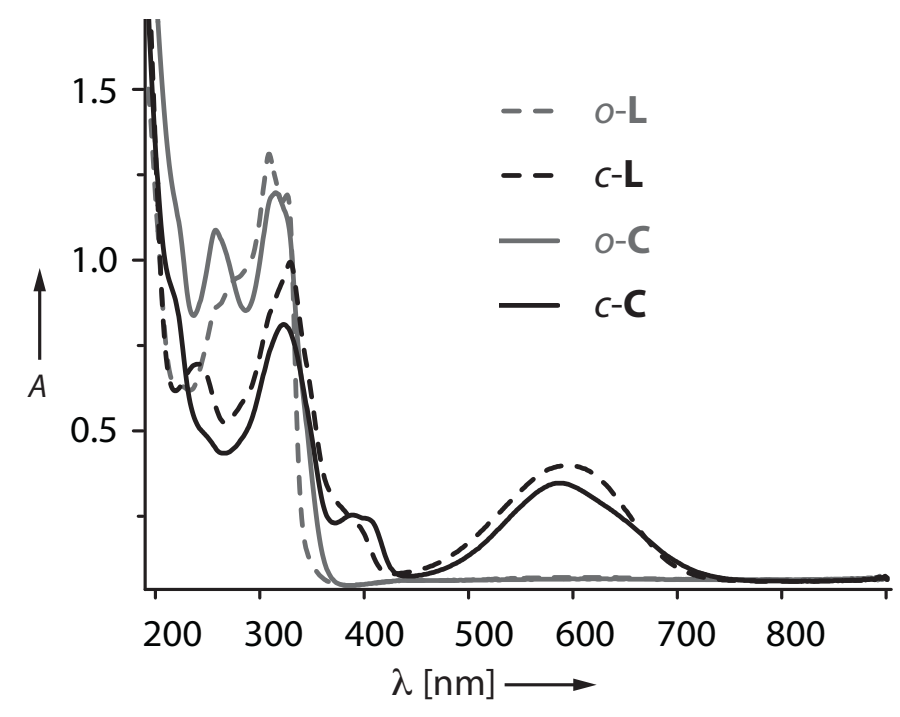

Figure 5-7 UV-Vis spectra of $o$-L, $c$-L, $o$-C and $c$-C. Copyright (C) 2013 WILEY-VCH Verlag GmbH \& Co.

The formation of $o-\mathrm{C}$ was also confirmed by FTICR ESI mass spectrometry. The signals of cage complex $o-C$ are centred around $m / z$ 623.8, 860.7 and 1334.0, which correspond to the differently charged cationic $[o-\mathrm{C}]^{4+},\left[o-\mathbf{C}+\mathrm{BF}_{4}\right]^{3+}$ and $\left[o-\mathrm{C}+2 \mathrm{BF}_{4}\right]^{2+}$ species. Their isotopically resolved patterns showed consistency with the formulation $\left[o-\mathbf{C}+n \mathrm{BF}_{4}\right]^{(4-n)+}$ $(n=0-2)$ along with peaks due to the fragmentation of the cage complexes (Figure 5-8).

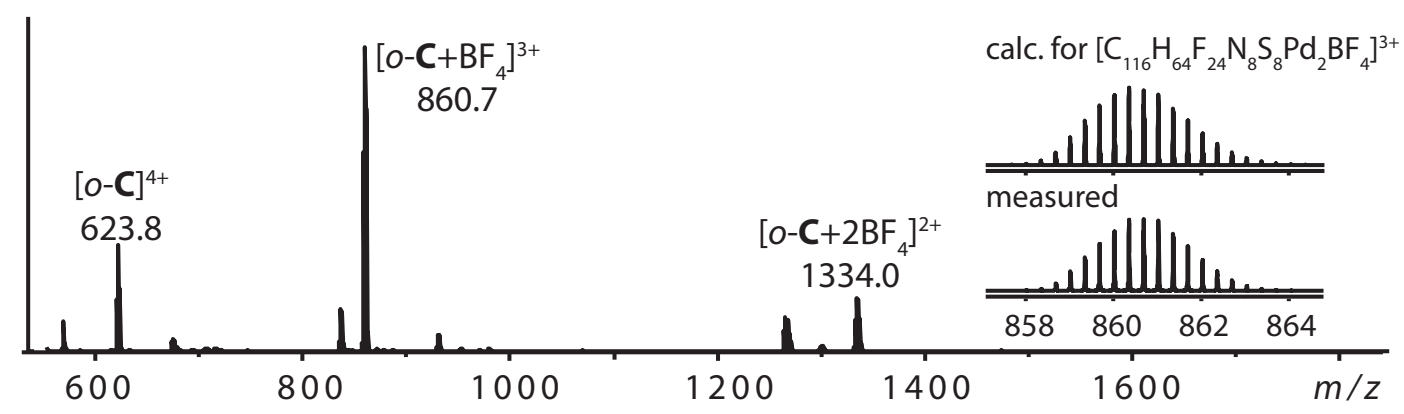

Figure 5-8 ESI mass spectra of $o$-C. Copyright (C) 2013 WILEY-VCH Verlag GmbH \& Co.

Interestingly, the ${ }^{1} \mathrm{H}-\mathrm{NMR}$ spectroscopic analysis revealed that the photocyclization conversion of $o$-C was slightly higher (> $96 \%$ ) than that of $o$-L (83\%) after equal irradiation times. In the cage complex $o$-C, all four ligands should adopt a $C_{2}$-symmetrical conformation, which brings the methyl-substituted thiophene carbon atoms into a favourable conformational position for the light-induced C-C bond formation. However, the same thiophene carbon atoms are not constrained in the free ligand $o$-L, so the equilibria between favourable and photo-inactive conformations are reported to affect the photocyclization rate.[69] 


\subsection{Symmetry interpretation}

\subsubsection{Symmetry-related interpretation of the NMR spectra}

Besides the notable chemical shifts upon the formation of closed-form cage compound $c$-C, additional splitting of each of the pyridyl signals was observed in the ${ }^{1} \mathrm{H}$ NMR spectrum. Protons $H_{a}, H_{b}, H_{c}$, and $H_{d}$ were split into three sets of signals with integral ratio $1: 2: 1$ (Figure 5-4 and Figure 5-9). The additional signal splitting could only be observed in the rigid $c$-C spectrum, but not in the flexible $o$-C spectrum, even when it was measured using a $900 \mathrm{MHz}$ spectrometer.
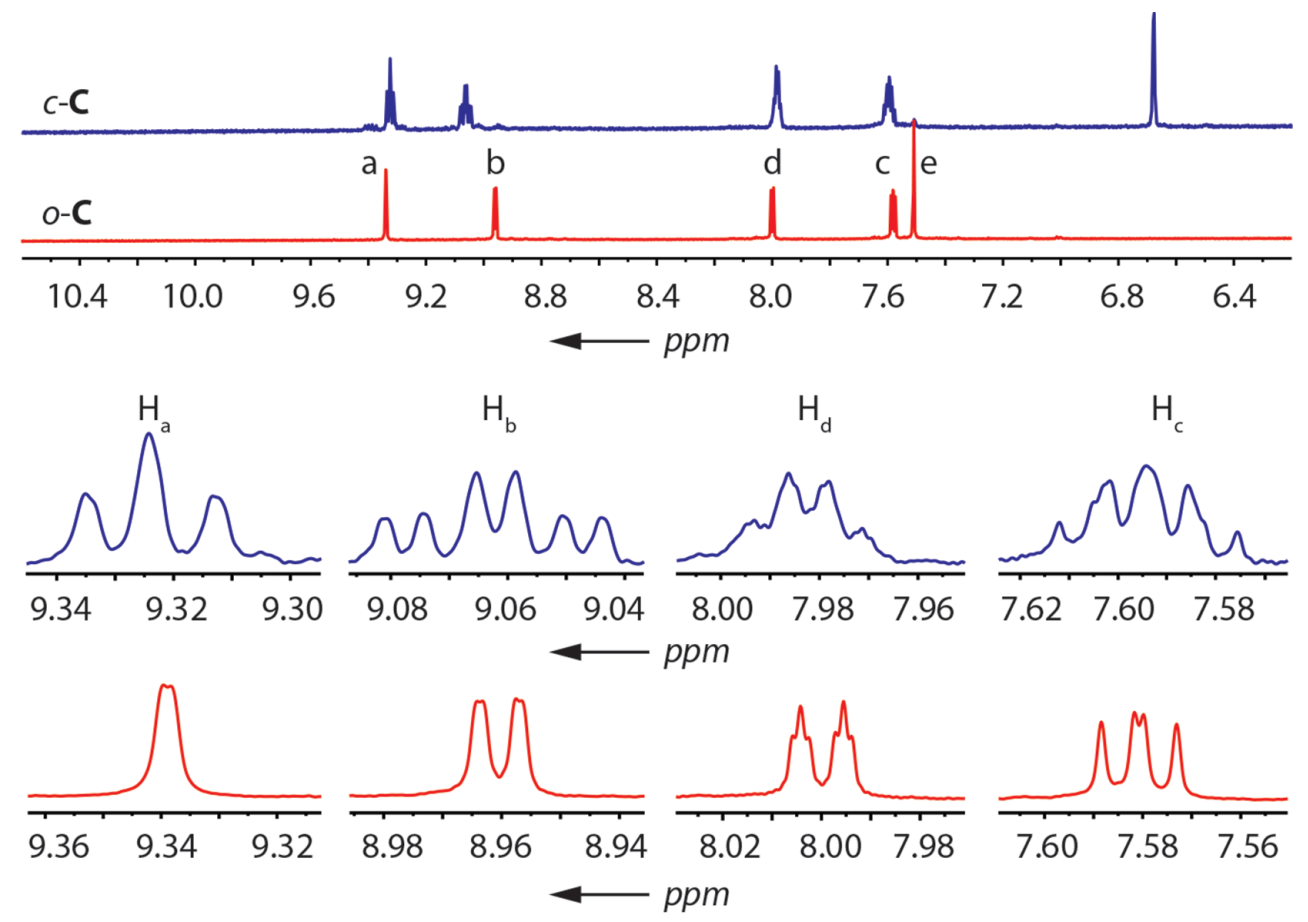

Figure 5-9 Top: ${ }^{1} \mathrm{H}$ NMR spectra $\left(900 \mathrm{MHz}, \mathrm{CD}_{3} \mathrm{CN}\right)$ of $o-\mathrm{C}$ (red) and $c$-C (blue) in the aromatic region. Bottom: enlarged view of the four pyridyl proton signals with the observed signal splitting of $c$-C.

In addition, the ${ }^{13} \mathrm{C}$ NMR spectrum allowed the differentiation of at least five peaks for some of the pyridine carbon atoms (Figure 5-10). In contrast to the signals of $o$-C, the pyridine signals of $c$-C show a splitting as expected for the non-interconverting set of stereoisomers. The ${ }^{1} \mathrm{H},{ }^{13} \mathrm{C}$ HSQC spectrum of $c$-C (Figure 5-11) showed that a quite large signal splitting (of more than three) exists actually in the ${ }^{1} \mathrm{H}$ NMR although it could not be clearly enough observed on a $900 \mathrm{MHz}$ spectrometer. 


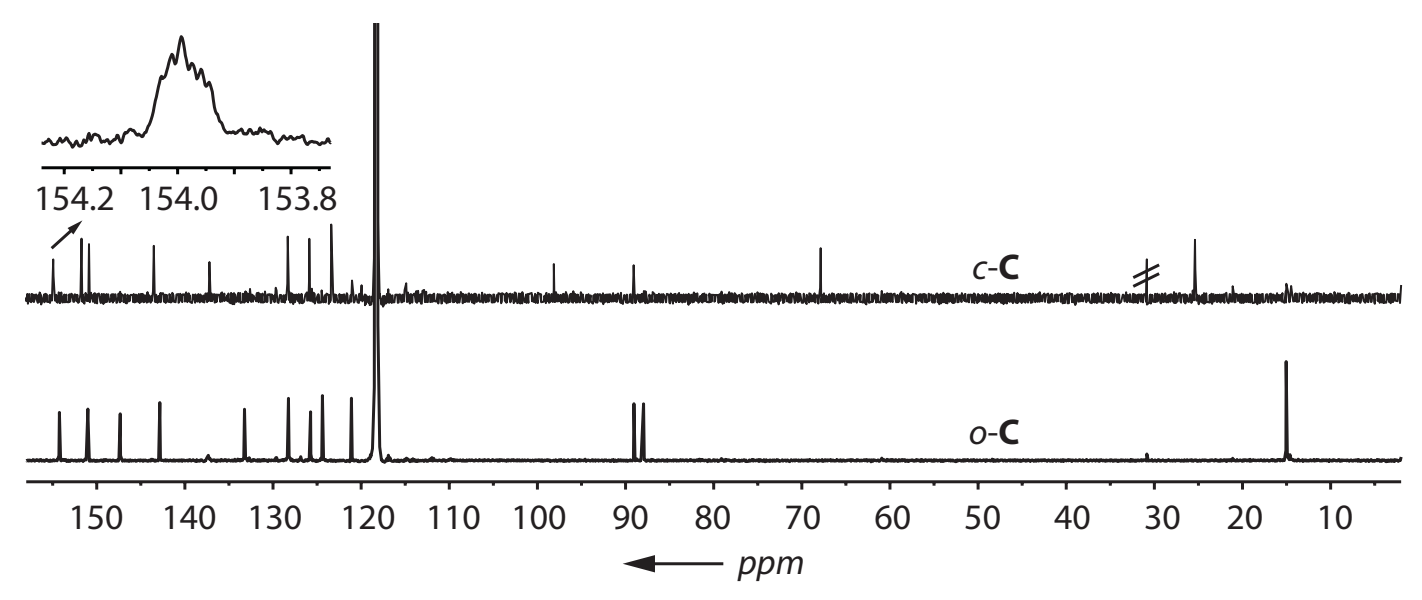

Figure 5-10 ${ }^{13} \mathrm{C}$ NMR spectra $\left(125 \mathrm{MHz}, \mathrm{CD}_{3} \mathrm{CN}, 300 \mathrm{~K}\right.$; inset: $\left.226 \mathrm{MHz}\right)$ of $o-\mathbf{C}$ and $c$-C.

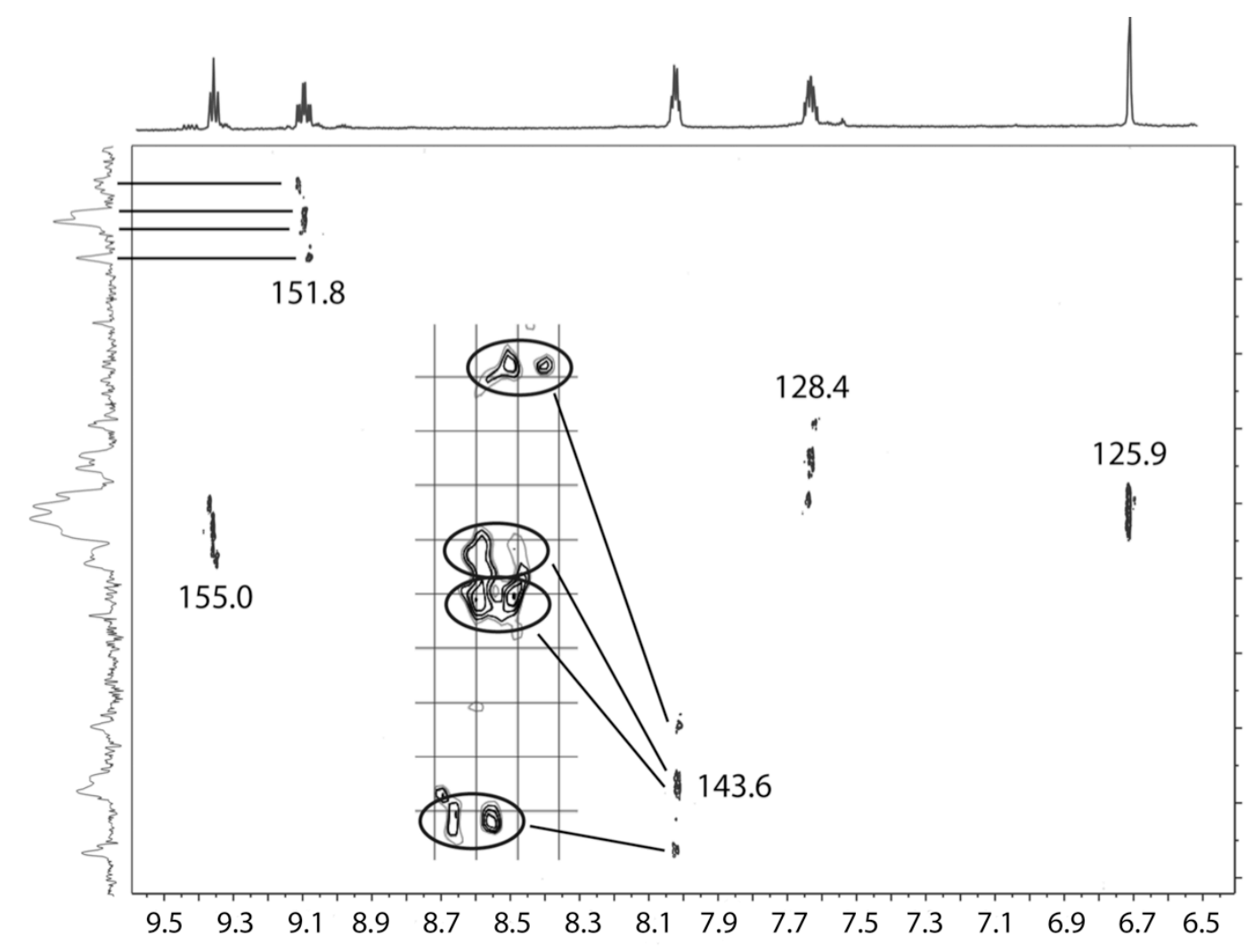

Figure 5-11 ${ }^{1} \mathrm{H}-13 \mathrm{C}$ HSQC spectrum $\left(900 \mathrm{MHz}, \mathrm{CD}_{3} \mathrm{CN}\right.$, aliased onto a spectral width of $0.5 \mathrm{ppm}$ ) of $c-\mathrm{C}$ with splitting of each ${ }^{13} \mathrm{C}$ signal (ppm assignments of the undistorted ${ }^{13} \mathrm{C}$ NMR spectrum are given next to the signal contours).

The ${ }^{1} \mathrm{H}$ NOESY spectrum showed that the pyridine protons, which gave the pseudo-triplets are in close contact with each other (Figure 5-12), which indicated that the signal splitting did not belong to three distinguishable cage isomers of $c$-C complex with 1:2:1 ratio. Rather, they belong to similar proton environments that are found throughout all possible cage diastereomers. 


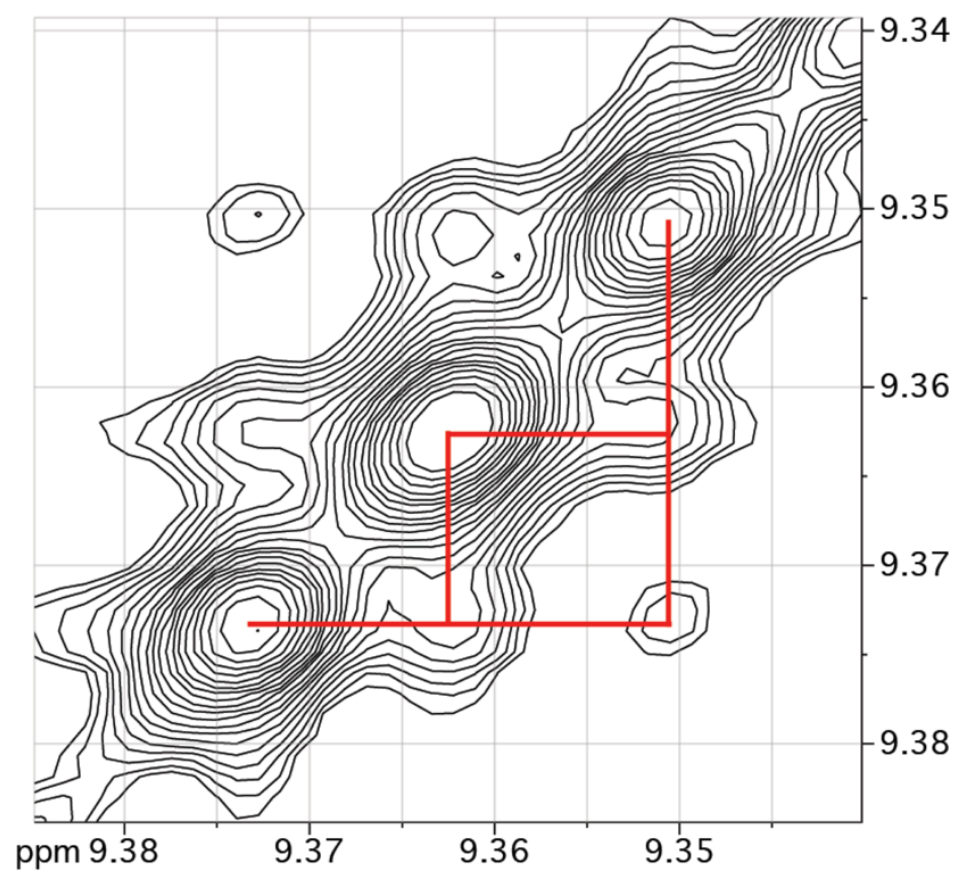

Figure 5-12 ${ }^{1} \mathrm{H}$ NOESY spectrum $\left(900 \mathrm{MHz}, \mathrm{CD}_{3} \mathrm{CN}\right)$ of $c$-C.

The most reasonable explanation for all the NMR spectroscopic observations is as follows:

1. The high flexibility of open cage $\boldsymbol{o}$-C structure enables the possibility to form different cage isomers (schematically depicted in Figure 5-13) in solution, which can interconvert between each other quickly by rotating along the cyclopentenethiophene single bonds. This is why only a single clean signal of each proton-, carbon-, and fluorine-atom was detected in the ${ }^{1} \mathrm{H},{ }^{13} \mathrm{C}$, and ${ }^{19} \mathrm{~F}$ NMR spectra.

2. The four closed-form $c$-L ligands around the Pd-centre might have different helical chiralities $\left(P\right.$ or $M$ ) because of the $C_{2}$-symmetry of the ligand. Possible stereoisomer included: two pairs of enantiomers (I) PPPP/MMMM; (II) $P M M M / M P P P$, two meso-forms (III) PMPM and (IV) PPMM. All the cage isomers, which have comparable energy (calculation in 4.3.2), are all populated in the solution at room temperature. According to a statistical analysis related to the assumption, the ratio between the four types of stereoisomers is:

(I) : (II) : (II) $:($ IV) $=(1+1):(4+4): 2: 4(\Sigma=16)$.

3. The pyridyl rings of the ligand, which coordinate around the palladium centres, must experience different chemical environment, therefore only the signals of these pyridyl rings presented remarkable splitting in the ${ }^{1} \mathrm{H}$ and ${ }^{13} \mathrm{C}$ NMR spectra. The splitting with the 1:2:1 ratio in the ${ }^{1} \mathrm{H}$ NMR and NOESY spectra encourages us to make a "next neighbour analysis" of all the pyridyl rings. The colour marked area in Figure 5-13 presents three chemical environments (a)-(c) respectively, 
which distinguished the stereochemical relationship between the central pyridyl ring and its neighbouring pyridyl rings.

(a) Both neighbouring ligands have the same chirality as the centre ligand

(b) Both neighbouring ligands have the opposite chirality as the centre ligand

(c) One neighbouring ligand has the same chirality, and the other shows the opposite

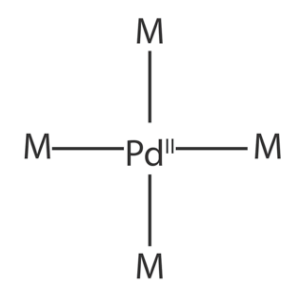

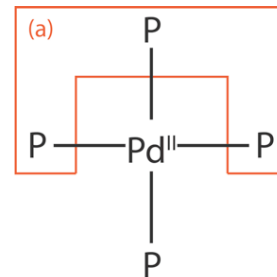

(I)

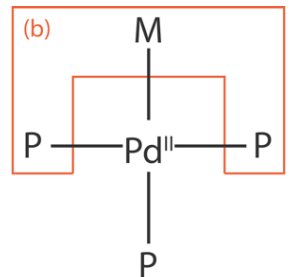

(II)

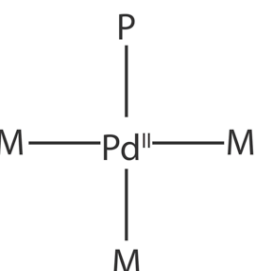

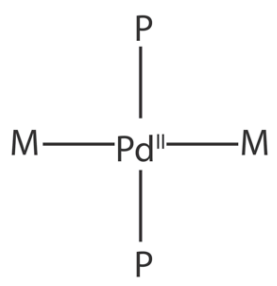

(III)

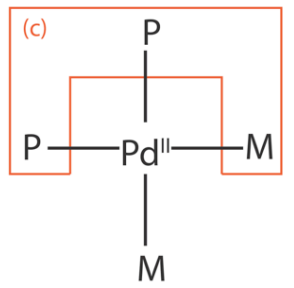

(IV)

Figure 5-13 Simplified illustration of the possible isomers of cage compounds $c$-C. The arrangement of the four ligands around the $\mathrm{Pd}^{I I}$ centres leads to the following cage stereoisomers: (I) PPPP and MMMM, (II) MPPP and PMMM, (III) meso-PMPM and (IV) meso-PPMM. Depicted is the view from the top of the cage molecule down the Pd-Pd-axis; colour marked areas show the different chemical environments around the Pd centre.

The three chemical environments (a)-(c) are the main factor, which affect the distinguishable peak splitting for each of the pyridyl ring signal in the ${ }^{1} \mathrm{H}$ NMR spectrum. The weak stereochemical influence of the last pyridyl ligand that is located opposite to the central ligand in the coloured area is responsible for the splitting observed in the ${ }^{13} \mathrm{C}$ and HSQC spectra. Taking into consideration the distribution of the chemical environments (a)-(c) including all the cage isomers and the statistical distribution of the isomers (I)-(IV), the signal intensities could be calculated.

- All sites of (I) and one quarter of each (II) structure share the same chemical environment (a): $1+1+4 \times 1 / 4+4 \times 1 / 4=4$.

- One quarter of each (II) structure and half of (III) have the chemical environment (b): $4 \times 1 / 4+4 \times 1 / 4+2 \times 1 / 2+2 \times 1 / 2=4$.

- Half of each (II) structure and all sites of (IV) give the (c) environment: $4 \times 1 / 2+4 \times$ $1 / 2+4=8$. 
In conclusion, the calculated ratio of (a) : (b) : (c) is $4: 4: 8=1: 1: 2$ confirms the $1: 2: 1$ splitting that was observed from the ${ }^{1} \mathrm{H}$ NMR spectrum.

\subsubsection{Calculated structure results of cages $c-\mathbf{C}$ and $o-\mathbf{C}$}

All four possible cage diastereomers of cage $c$-C (two pairs of enantiomers $P M M M / M P P P$, two meso-forms PPMM and PMPM) were built using the software Spartan '08[78] and were optimized roughly using molecular mechanics methods (MMFF). Then, the first structural refinement of each cage isomer was optimized on semiempirical PM6 $(+4$ positive charged, spin multiplicity 1 , no constraints, default convergence criteria, no imaginary frequencies observed) and DFT (B3LYP/LANL2DZ) level of theory. ${ }^{[79]}$ All tetracationic $c$-C cage isomers are of comparable stability and energetically comparable, the energy difference between all isomers is approximate $3 \mathrm{~kJ} \mathrm{~mol}^{-1}$ (Figure 5-14).

For the corresponding isomeric structure of $o-\mathbf{C}$, similar results were obtained. Therefore we surmise that the $o$-C cage is composed of a mixture of all four isomers as well, which is exchanging fast on the NMR time scale. Since the disassembly and reassembly rate of the cage (between ligands and the palladium cations) in acetonitrile is on a longer time scale (several minutes) at room temperature,[70] we believe that the equilibrium is achieved by rotations around the carbon-carbon single bonds connecting the perfluorocyclopentene and the thiophene rings of $o-\mathbf{C}$ (red arrows show the rotation directions in Figure 5-3a).

The aforementioned bond rotation of $\boldsymbol{o}$-C will no longer be possible as soon as the photocyclization occurs upon the irradiation with UV $365 \mathrm{~nm}$. Once the closed-ring form cage $c$-C was formed, the individual cage isomers (I) - (IV) turn out to be distinguishable in the NMR measurements.

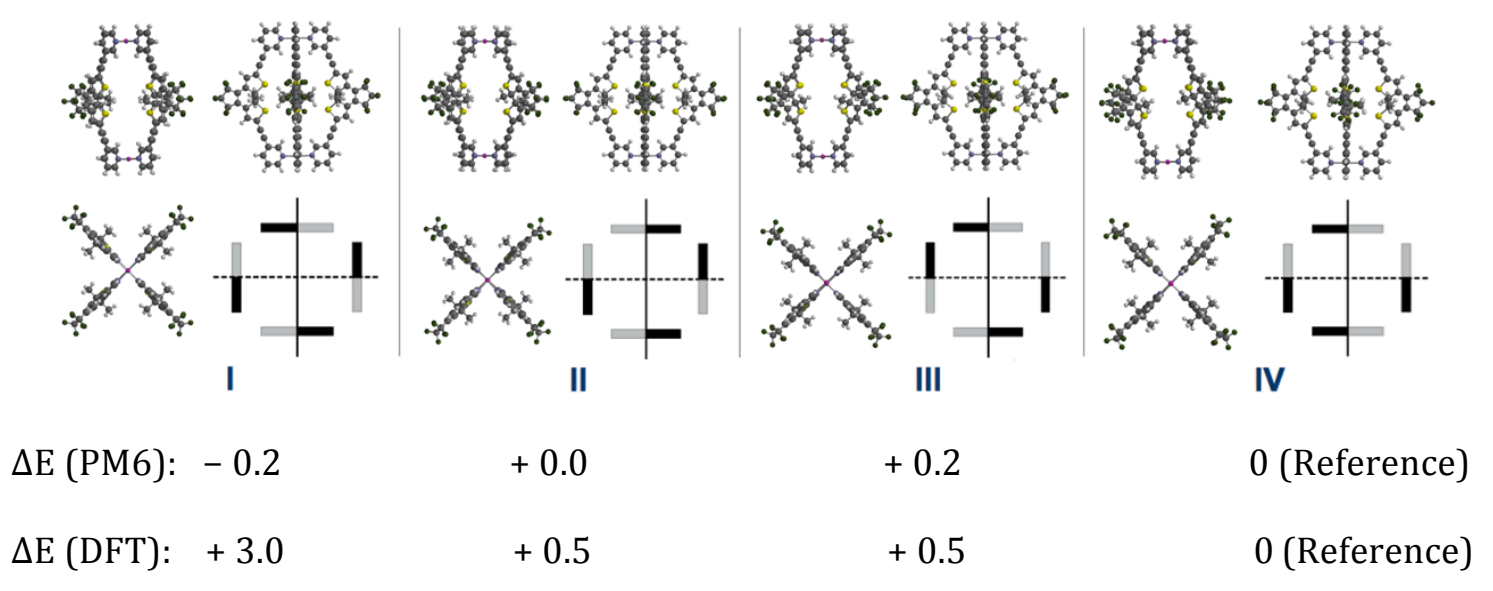

Figure 5-14 The DFT (B3LYP/LANL2DZ) geometry optimization of the four possible cage isomers, (I) PPPP, (II) PPPM, (III) PMPM and (IV) $P P M M$. Energies are given in $\mathrm{kJ} \mathrm{mol}^{-1}$ relative to isomer IV. 


\subsubsection{X-ray data of the cage $o-\mathrm{C}$}

The X-ray structure was measured by Dr. Jakob Hey, solved and refined by Reent Michel.

Suitable single crystals of the $\mathrm{BF}_{4}^{-}$salt of $o$-C were obtained through solvent diffusion of $o$ dichlorobenzene into an acetonitrile solution of $o$-C (Figure 5-15). The crystal covered with inert polyether oil[80-82] and placed on a Bruker D8 fixed-angle microcrystal diffractometer, which equipped with a liquid helium Oxford Cryostems and an APEXII CCD detector. The single crystal data was collected at the ChemMatCARS Beamline (15-ID-B) at the Advanced Photon Source (APS). Data integration and absorption correction were undertaken with the software SAINT[83] and SADABS.[84] Each independent hkl-file was combined together and the space group was determined with the program XPREP. The structure was solved by using SHELXS-97 then was refined and extended with SHELXL2012/4 beta test version.[85]


Figure 5-15 a)-c) X-ray structure of cage $o$-C shown from different perspectives (C: grey, H: white, N: blue, B: brown, F: green, S: yellow, Pd: purple. Solvent molecules omitted). d) Schematic depiction of the stereochemical relationships between the four ligands viewed from top (black/grey bars: close/remote methyl groups). Copyright (C 2013 WILEY-VCH Verlag GmbH \& Co. 
Interestingly, only one of the four possible stereoisomers was crystalized. The structure was confirmed by two separate measurements with two individual crystalline samples of $o$-C. The only structure we observed in the solid-state is the meso-isomer PPMM (IV) consisting both of the enantiomeric ligands, which consists a total $C_{2 \mathrm{~h}}$ symmetry, that means the resulting cage structure is not chiral and the crystallization undergoes a chiral self-discrimination process. ${ }^{[75,76]}$

Indeed, two cells are possible for further integration and refinement. The lattice exceptions for the larger cell $17.3 \AA, 19.3 \AA$, $31.3 \AA, 91.52^{\circ}, 90.77^{\circ}, 91.94^{\circ}$ revealed a typical A-centered unit cell. After integrating the smaller cell, lower R-values were received after the structure refinement; hence the smaller transformed cell was preferred. A summary of data collection and refinement for the smaller cell of $\boldsymbol{o}$-C complex is presented in Table 5-1.

Table 5-1 Crystal data and structure refinement.

\begin{tabular}{|l|l|}
\hline Empirical formula & $\mathrm{C}_{206} \mathrm{H}_{124} \mathrm{~B}_{4} \mathrm{Cl}_{30} \mathrm{~F}_{40} \mathrm{~N}_{8} \mathrm{~S}_{8} \mathrm{Pd}_{2}$ \\
\hline $\mathrm{M}[\mathrm{g} / \mathrm{mol}]$ & 5047.14 \\
\hline $\mathrm{T}[\mathrm{K}]$ & $15(2)$ \\
\hline$\lambda[\AA ̊ \Omega]$ & 0.39360 \\
\hline Crystal system & Triclinic \\
\hline Space group & $P \overline{1}$ \\
\hline Unit cell dimensions & $a=17.314(2) \AA$ \\
\hline & $b=18.193(2) \AA$ \\
\hline & $c=18.612(3) \AA$ \\
\hline & $5236.3(12) \AA^{3}$ \\
\hline Volume & 1 \\
\hline $\mathrm{Z}$ & 1.601 \\
\hline$\rho_{\text {calc. }\left[\mathrm{Mg} / \mathrm{m}^{3}\right]}$ & 0.324 \\
\hline$\sigma\left[\mathrm{mm}{ }^{-1}\right]$ & 2526 \\
\hline$F(000)$ & $0.200 \times 91.705(2)^{\circ}$ \\
\hline Crystal size [mm $\left.{ }^{3}\right]$ & 0.969 to $15.246^{\circ}$ \\
\hline$\theta-$ range & $-23 \leq h \leq 23$ \\
\hline Index ranges & $-23 \leq l \leq 24$ \\
\hline Reflections collected & 92627 \\
\hline Independent reflections & $24981\left[R_{\text {Int. }}=5.33 \%\right]$ \\
\hline Completeness to $\theta=13.660^{\circ}$ & $96.9 \%$ \\
\hline
\end{tabular}




\begin{tabular}{|l|l|}
\hline Absorption correction & Semiempirical from equivalents \\
\hline Max. and min. transmission & 0.7437 and 0.6692 \\
\hline Refinement method & Full-matrix least-squares on $F^{2}$ \\
\hline Data / restraints / parameters & $24978 / 10822 / 2533$ \\
\hline Goodness-of-fit on $F^{2}$ & 1.019 \\
\hline Final $R$ indices $[I>2 \sigma(I)]$ & $R_{1}=0.0598, w R_{2}=0.1462$ \\
\hline$R$ indices (all data) & $R_{1}=0.0791, w R_{2}=0.1603$ \\
\hline Extinction coefficient & 0 \\
\hline Largest diff. peak and hole $[\mathrm{e} \AA-3]$ & 2.163 and -0.988 \\
\hline
\end{tabular}

All four palladium cations are square-planar coordinated by four pyridyl ligands with Pd-N distances from 2.017 to $2.106 \AA$. Two $\mathrm{BF}_{4}{ }^{-}$anions are encapsulated in the cage between the two palladium cations, and another two $\mathrm{BF}_{4}{ }^{-}$anions are located near the outer faces of the palladium cations. The shortest Pd-F distance from the palladium cation to the outer $\mathrm{BF}_{4^{-}}$is $3.047 \AA$, and to the inner $\mathrm{BF}_{4}^{-}$is $3.077 \AA$, which matches quite well with our previous work.[58-66] The distance between two palladium cations is $16.751 \AA$ A. The two $\operatorname{Pd}(\text { pyridine })_{4}$-planes are parallel to each other but are not directly overlapping (from Figure 5-15b). The crystal packing effects probably cause this skewed conformation of the cage structure. This also indicates the conformational flexibility of the ligand structure $o$-L. The two pairs of the opposite located ligands are in different conformations (Figure $\mathbf{5 - 1 5 c}$ ), one pair of the ligands is distorted. The dihedral angles between the $C$ - $C$ bonds of cyclopentene and thiophene rings are $42^{\circ}$ and $48^{\circ}$ of each pair of ligands in the X-ray structure. And this differs from the DFT optimized results of the corresponding closed-ring PPMM c-C isomer, which is rigid, and not skewed, the Pd-Pd axis orthogonal to both of the Pd(pyridine) 4 -planes (Figure 5-14). 

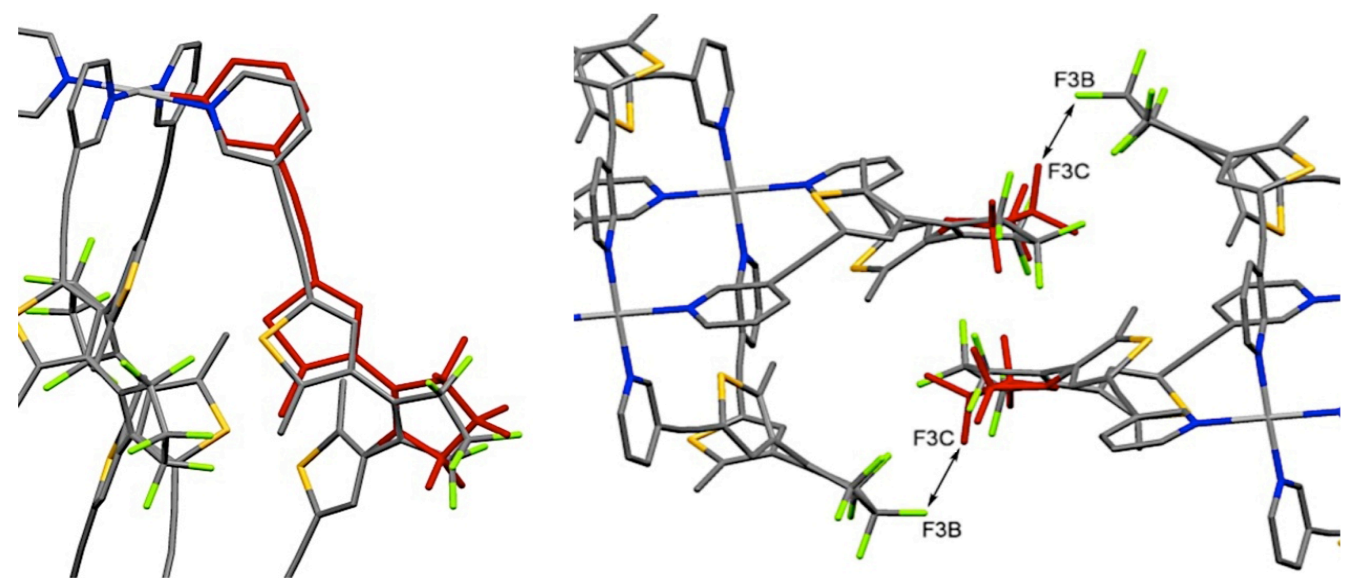

Figure 5-16 Left: disorder of the perfluorocyclopentene ring with one sidearm $440 \%$, drawn in red). Right: two neighbouring cage-structures, in which F3B and F3C were shown.

Interestingly, an unusually short distance (2.43 $\AA$ ) between two fluorine atoms (F3B and F3C in Figure 5-16) of two neighbouring cages was also observed. The asymmetric unit was constructed from one palladium cation ( $\mathrm{PdI}$ ) and two ligands, as well as lattice solvent and counteranions $\mathrm{BF}_{4}^{-}$. One of the ligands is disordered, which influences the perfluoropentene $\mathrm{C}_{5} \mathrm{~F}_{6}$-unit with one sidearm (marked in red, Figure 5-16) and caused it to distribute over two positions with a site occupation factor of 40 and $60 \%$. One cage structure interlocks the $\mathrm{C}_{5} \mathrm{~F}_{6}$-unit of the adjacent cage structure and leads to a close proximity of the fluorine atoms. Only the minor part of the disordered $\mathrm{C}_{5} \mathrm{~F}_{6}$-unit gets close to F3B of the not disordered ligand. Similar intermolecular F-F distances (between 2 and $2.43 \AA$ ) could also be found in the Cambridge Structure Database (CSD) ${ }^{[86]}$ in 349 hits (CSD version 5.33, Feb. 2012). Not surprisingly, a number of $o$-dichlorobenzene molecules that was used in the vapor diffusion crystallization setup were trapped in the crystals (Figure 5-17). 

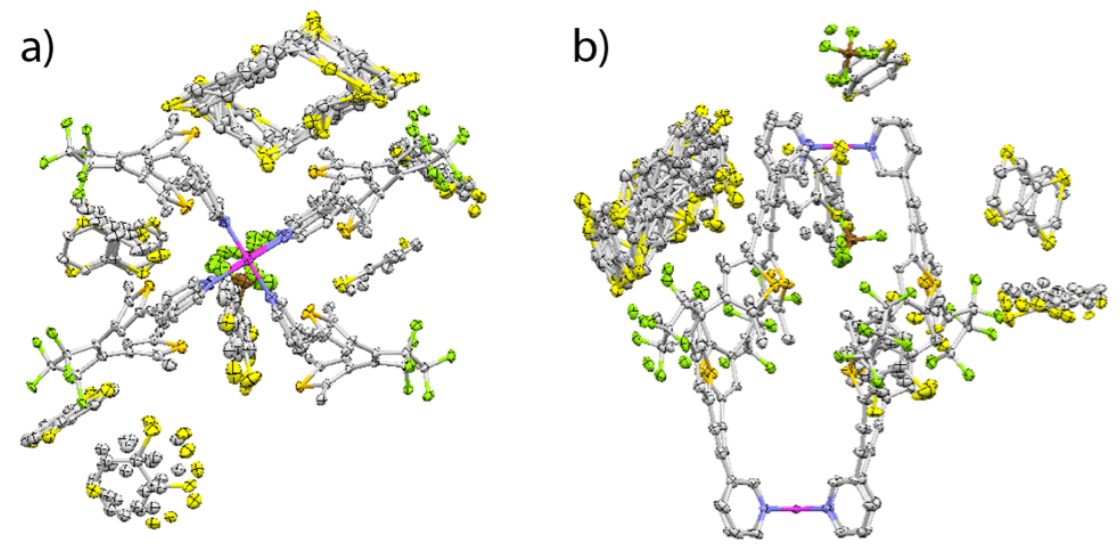

c)

d)
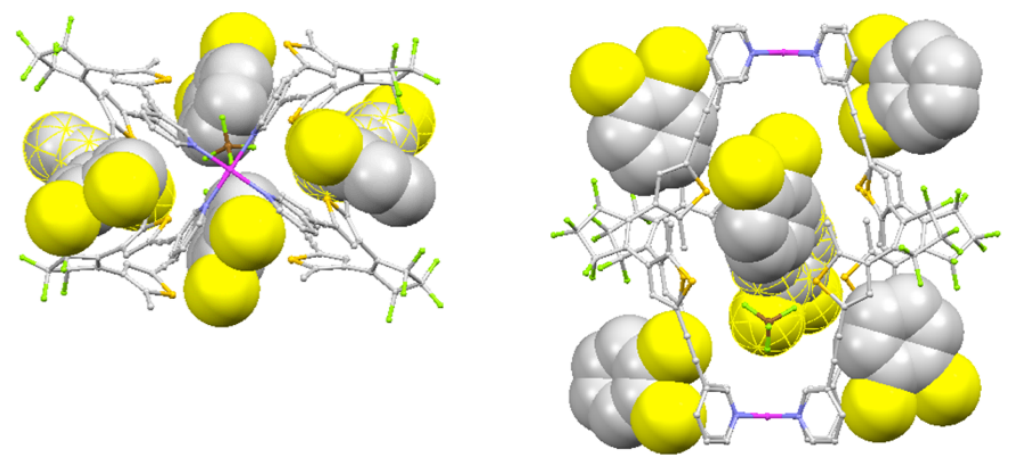

Figure 5-17 Structure of $o$-C, showing 50\% probability ellipsoids. a) top view and b) side view (H atoms are omitted for clarity. C: grey, N: blue, Pd: pink; S: orange; B: brown, F: green, $\mathrm{Cl}$ : yellow). c) top and d) side view of the structure with all the $o$-dichlorobenzene molecules highlighted as spacefilling representations that are in close contact to the cage structure (in case of overlapping, the more remote structure is indicated by a fine yellow pattern; most counter anions and surrounding solvent molecules are omitted).

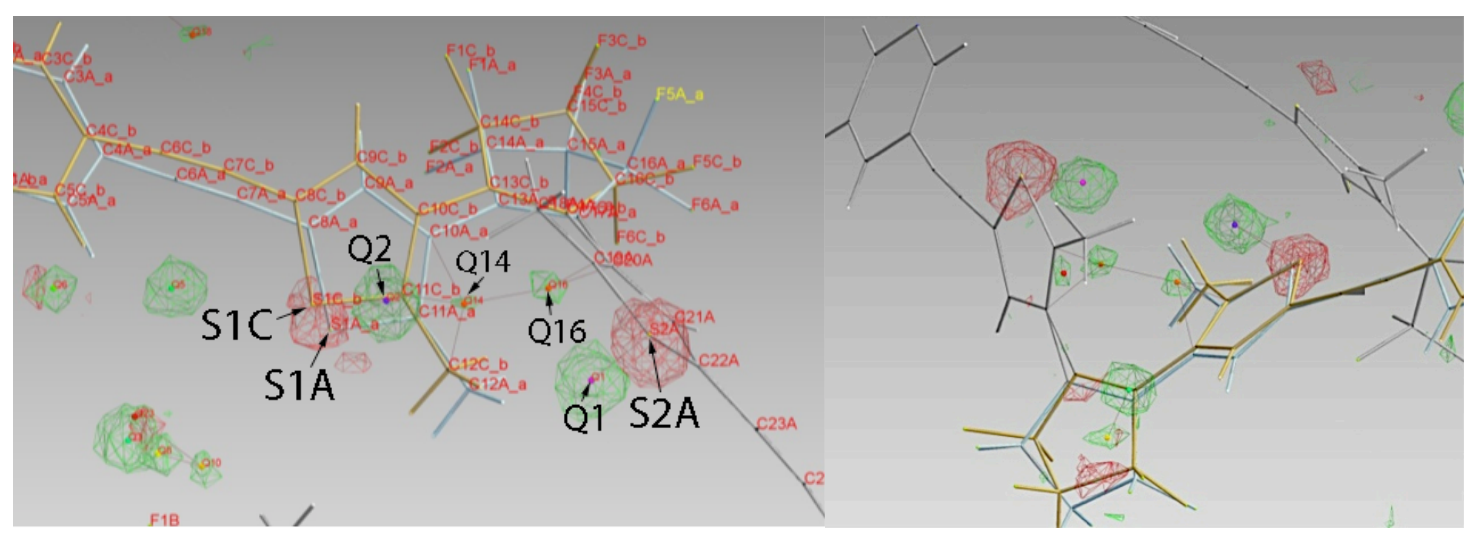

Figure 5-18 Photoswitch fragment with indication of disorder between the switched and unswitched form. The $F_{0}-F_{C}$ map visualizes positive residual electron density in green and negative in red. 
The two highest residual density peaks are found close to the sulphur atoms S2A and S1A/S1C (Figure 5-18). The electron density holes at the sulphur atoms demonstrate exactly the disorder of the thiophene parts. This disorder was caused by the coexistence of the switched and unswitched form of the ligand. The residual density peaks Q1 and Q2 show the position for the sulphur atoms of the thiophene ring and peaks Q14 and Q16 give the position of carbon atoms of the new-formed six-membered ring both after the photocyclization. Further refinement was not possible because of the low amount of describable residual electron density.

\subsection{Host-Guest interaction}

In order to understand the cage structures in the solution and the photoswitching processes better, diffusion-ordered NMR spectroscopy (DOSY) of both photoisomers (o-C and $c$-C) was measured (Figure 5-19). The DOSY spectra showed a single band of $o-\mathbf{C}$ at $\log D=-9.08$, which is clearly smaller than the other single band of $c-C$ with $\log D$ value of -9.16 in $\mathrm{CD}_{3} \mathrm{CN}$. This means upon the photocyclization of the ligand backbones, the two photoisomers $o-\mathbf{C}$ and $c$-C differed from each other in size (hydrodynamic radii $r_{\mathrm{h}}$, Figure $\mathbf{5 - 1 9}$ ). The conformational flexibility of the $o$ - $\mathbf{L}$ is again the explanation of the smaller size of open-ring cage complex $o-\mathbf{C}$ in contrast to the stretched rigid ring closure structure $c$-C.

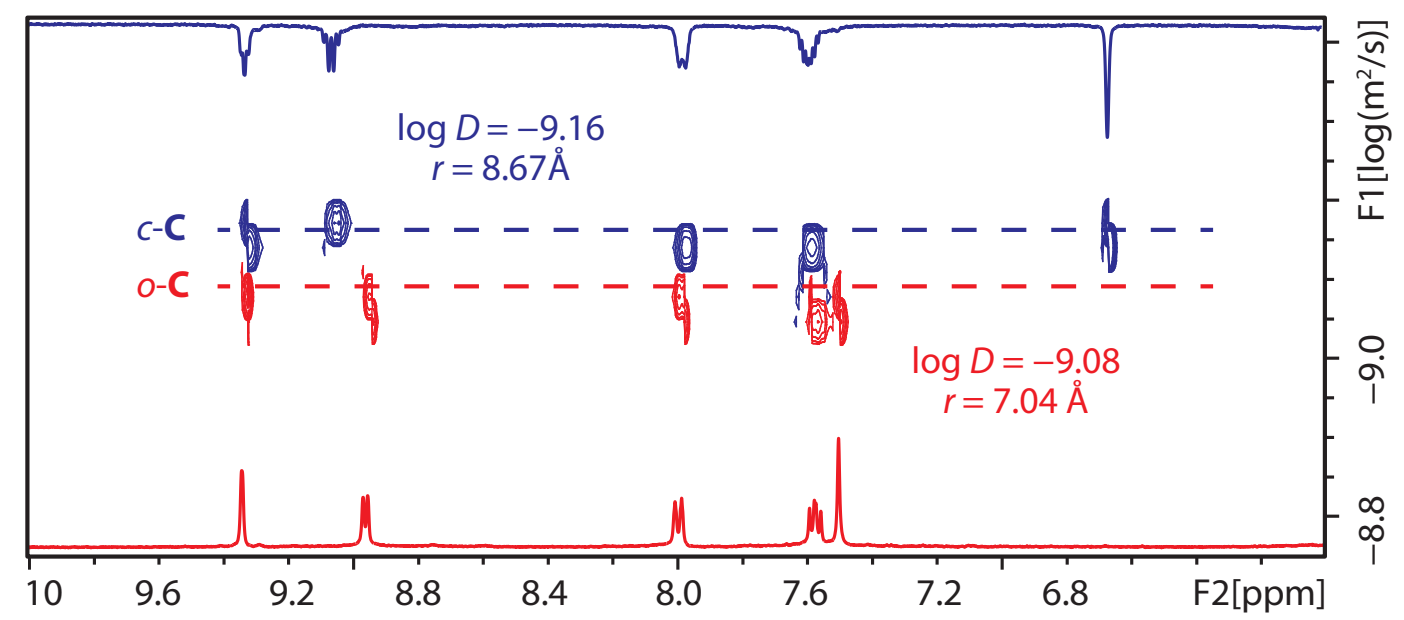

Figure 5-19 Superimposed 2D-DOSY spectra (400 MHz, $\left.\mathrm{CD}_{3} \mathrm{CN}\right)$ of $o-\mathbf{C}$ and $c$-C.

The reversible interconversion between the two cages with different sizes allows us to investigate the anion binding capability of the cages. We selected the spherical anion[73] dodecafluorododecaborate $\left[\mathrm{B}_{12} \mathrm{~F}_{12}\right]^{2-}$ as guest molecule $\mathbf{G}$, due to its appropriate size and the perfluorinated feature for sensitive ${ }^{19} \mathrm{~F}$ NMR measurement. 

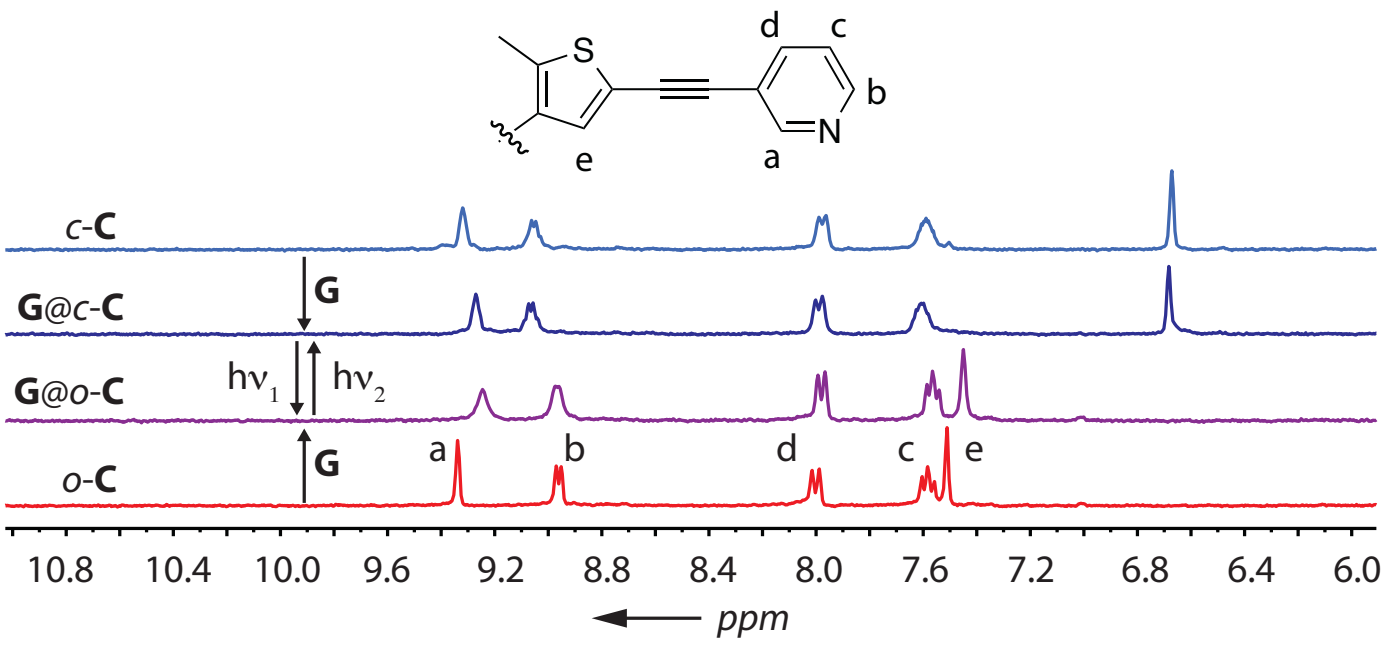

Figure 5-20 ${ }^{1} \mathrm{H}$ NMR spectra $\left(300 \mathrm{MHz}, \mathrm{CD}_{3} \mathrm{CN}\right)$ show the interconversion between $o-\mathrm{C}, c$ C, G@o-C, and G@c-C by irradiation and guest binding ( $h v_{1}=$ white light, $h v_{2}=365$ nm UV light).

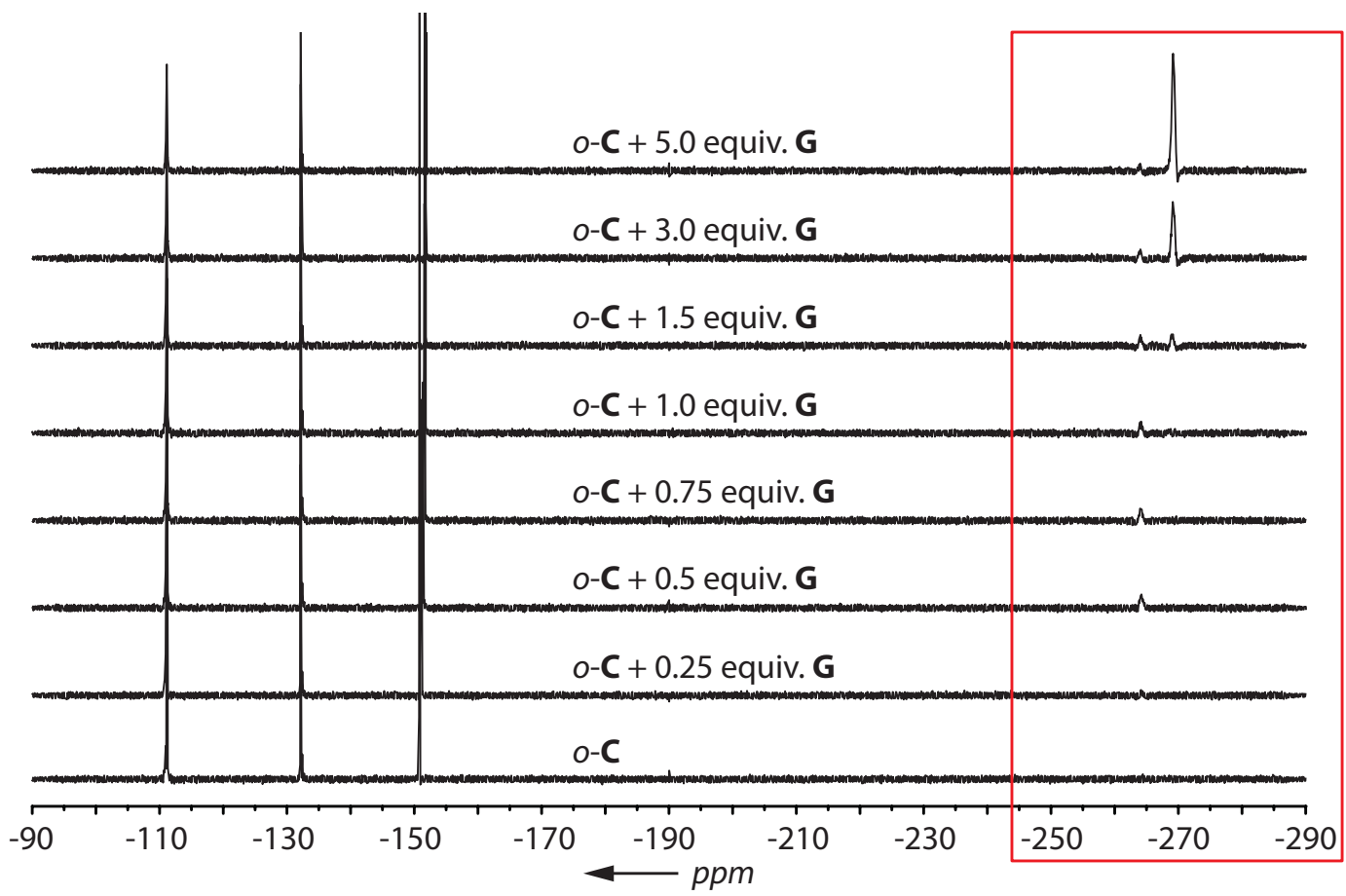

Figure 5-21 ${ }^{19} \mathrm{~F}$ NMR spectra of a simple titration of $\boldsymbol{o}$-C with $\mathbf{G}$. Encapsulated $\left[\mathrm{B}_{12} \mathrm{~F}_{12}\right]^{2-}$ signal and free $\left[\mathrm{B}_{12} \mathrm{~F}_{12}\right]^{2-}$ signal with an excess of guests were indicated in red square.

Adding the guest $\mathbf{G}$ solution to $o-\mathbf{C}$ or $c$ - $\mathbf{C}$ lead to significant signal shifts of the cage protons, especially the $\mathrm{H}_{a}$ protons of the pyridines, which were pointed inside the cage. However, the $\mathrm{H}_{\mathrm{a}}$ protons shifted upfield upon guest encapsulation (Figure 5-20), which differed from the previously reported downfield shifts of the pyridine $\mathrm{H}_{\mathrm{a}}$ protons in similar palladium pyridyl ligands-coordinated cages upon binding halides ${ }^{[66]}$ or sulphonates.[74] 
We suppose the hydrogen bonded solvent acetonitrile molecules were replaced by the large spherical guest anion that carries two delocalized negative charges, and is the reason for the upfield signal shift. The ${ }^{19} \mathrm{~F}$ NMR titration spectra of $o-\mathbf{C}$ with $\mathbf{G}\left[\mathrm{B}_{12} \mathrm{~F}_{12}\right]^{2-}$ presented a typical slow exchange of the encapsulated guest $\left[\mathrm{B}_{12} \mathrm{~F}_{12}\right]^{2-}$ (Figure 5-21). The slightly broadened signal of encapsulated $\left[\mathrm{B}_{12} \mathrm{~F}_{12}\right]^{2-}$ at $-264.2 \mathrm{ppm}$ showed a downfield shift $(\Delta \delta=$ $5.2 \mathrm{ppm}$ ) from the free $\left[\mathrm{B}_{12} \mathrm{~F}_{12}\right]^{2-}$ signal. The encapsulation process of the guest inside the cavity of both cages did not strongly influence to the photochemical interconversion between G@o-C and G@c-C.

Comparison of the hydrodynamic radii obtained from DOSY NMR spectra of all four complexes measured in $\mathrm{CD}_{3} \mathrm{CN}$ indicated an increase of the size each cage complex $o-\mathrm{C}$ and $c$-C after encapsulating the guest molecule G (Figure 5-22). Calculated hydrodynamic radii are listed in Table 5-2.

Hydrodynamic radii can be calculated from the diffusion values using the Stokes-Einstein equation:

$$
r=\frac{k \cdot T}{6 \cdot \pi \cdot \eta \cdot D}
$$

With $r=$ radius, $k=$ Boltzmann const., $T=$ temperature, $\eta=$ dynamic viscosity of $\mathrm{CD}_{3} \mathrm{CN}$ $\left(3.69 \times 10^{-4} \mathrm{~Pa} / \mathrm{s}\right)^{[87]}$ and $D=$ diffusion values estimated by the DOSY experiment (Figure 5-22).

Table 5-2 Calculated hydrodynamic radii for cage complexes and host-guest complexes.

\begin{tabular}{|c|c|c|c|c|}
\hline complex & $o-\mathbf{C}$ & $c-\mathbf{C}$ & G@o-C & G@c-C \\
\hline $\log D$ & -9.08 & -9.16 & -9.14 & -9.20 \\
\hline$D\left[\times 10^{-10} \mathrm{~m}^{2} \mathrm{~s}^{-1}\right]$ & $8.40 \pm 0.1$ & $6.80 \pm 0.1$ & $7.20 \pm 0.05$ & $6.25 \pm 0.05$ \\
\hline$r[\AA]$ & 7.04 & 8.67 & 8.16 & 9.33 \\
\hline
\end{tabular}




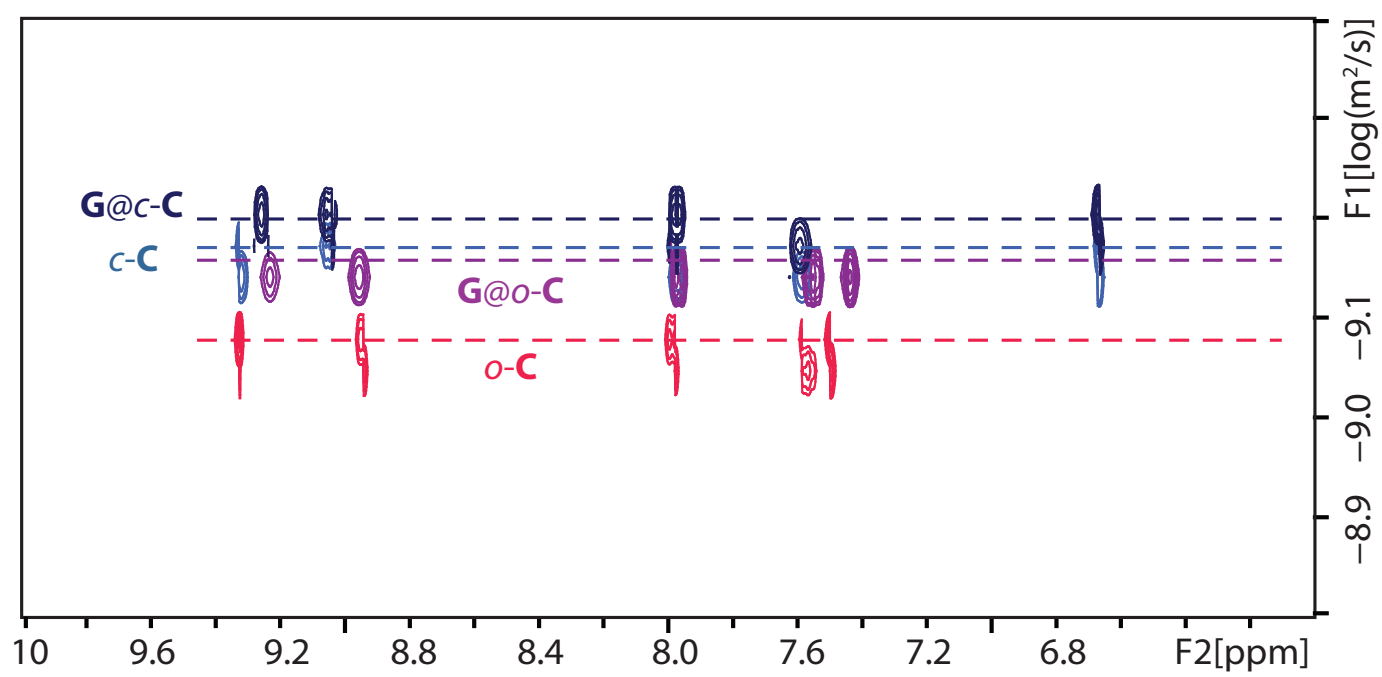

Figure 5-22 2D-DOSY spectra $\left(400 \mathrm{MHz}, \mathrm{CD}_{3} \mathrm{CN}\right)$ of cage complexes $o-\mathrm{C}, c$-C and hostguest complexes G@o-C, G@c-C.

The high resolution ESI mass spectrum also confirmed the encapsulation of G@o-C by observation of a peak at $m / z=1426.1$ resulting from $[\mathbf{G} @ o-\mathbf{C}]^{2+}$, which further supports the formation a $1: 1$ host-guest complex (Figure 5-23).

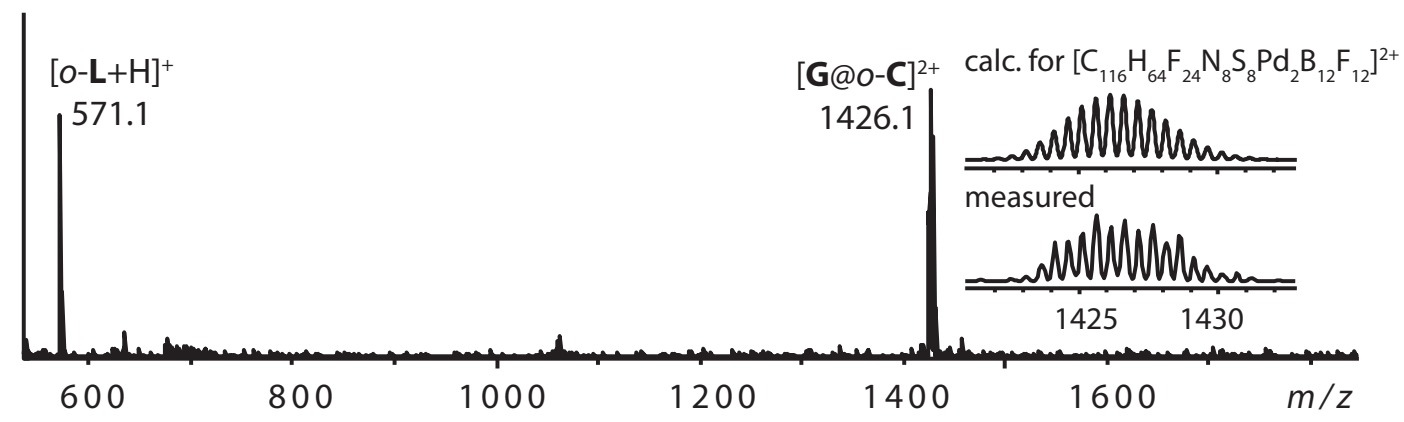

Figure 5-23 ESI mass spectra G@o-C.

According to the experimental results, both $1: 1$ encapsulation processes are showed as follows:

$$
\begin{aligned}
& o-\mathrm{C}+\mathrm{G} \rightleftharpoons \mathrm{G} @ o-\mathrm{C} \\
& c-\mathrm{C}+\mathrm{G} \rightleftharpoons \mathrm{G} @ c-\mathrm{C}
\end{aligned}
$$

In order to determine the binding constants of both host-guest complexes G@o-C and G@c-C, the encapsulation was monitored by ${ }^{1} \mathrm{H}$ NMR titrations, which were recorded at various temperatures $333 \mathrm{~K}, 313 \mathrm{~K}, 293 \mathrm{~K}, 273 \mathrm{~K}$. The binding constants of the host-guest complexes G@o-C and G@c-C were determined from the titration data by a non-linear regression approach using Thordarson's Matlab script[71] for fitting $1: 1$ equilibria to NMR data (Figure 5-24, Figure 5-25). 
a) +11 eq. $\mathbf{G}$

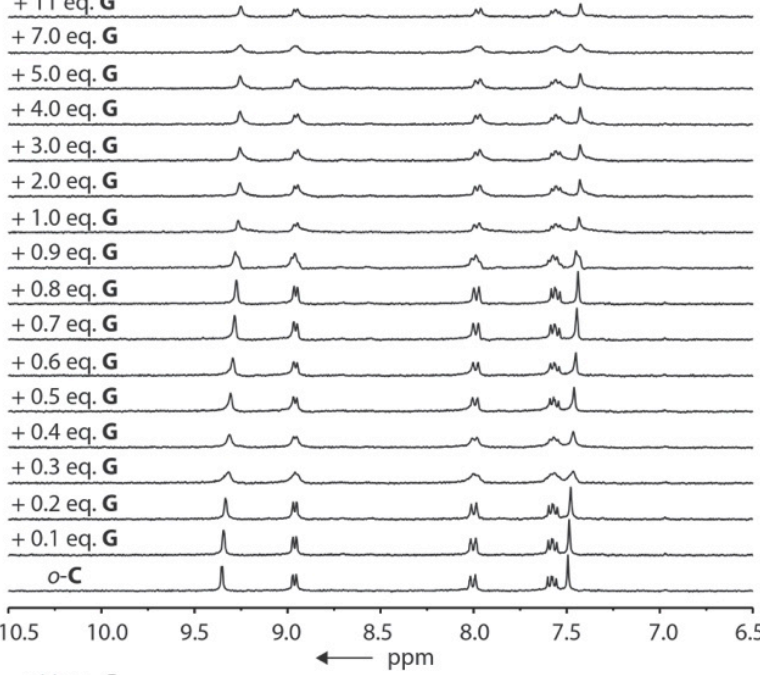

b) +11 eq. $\mathbf{G}$

+5.0 eq. G $\mathbf{G}$

+3.0 eq. G

+2.0 eq. $\mathbf{G}$

+1.0 eq. G

+0.9 eq. G

+0.8 eq. G

+0.7 eq. $\mathrm{G}$

+0.6 eq. $\mathbf{G}$

+0.5 eq. G

+0.4 eq. $\mathbf{G}$

+0.3 eq. G

+0.2 eq. $\mathbf{G}$

+0.1 eq. G

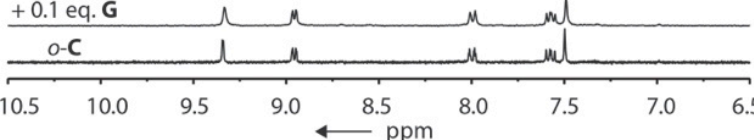

c) +11 eq. $\mathbf{G}$

+7.0 eq. G

+5.0 eq. G

+4.0 eq. G

+3.0 eq. G

+2.0 eq. G

+1.0 eq. G

+0.9 eq. G

+0.8 eq. $\mathbf{G}$

+0.7 eq. G

+0.6 eq. $\mathbf{G}$

+0.5 eq. $\mathbf{G}$

+0.4 eq. G

$+0.3 \mathrm{eg} . \mathrm{G}$

+0.2 eg. G

+0.1 eq. $\mathbf{G}$

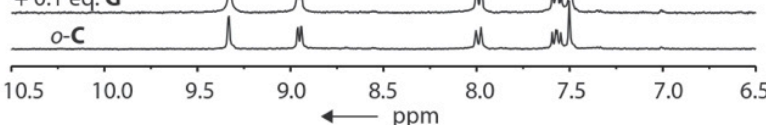
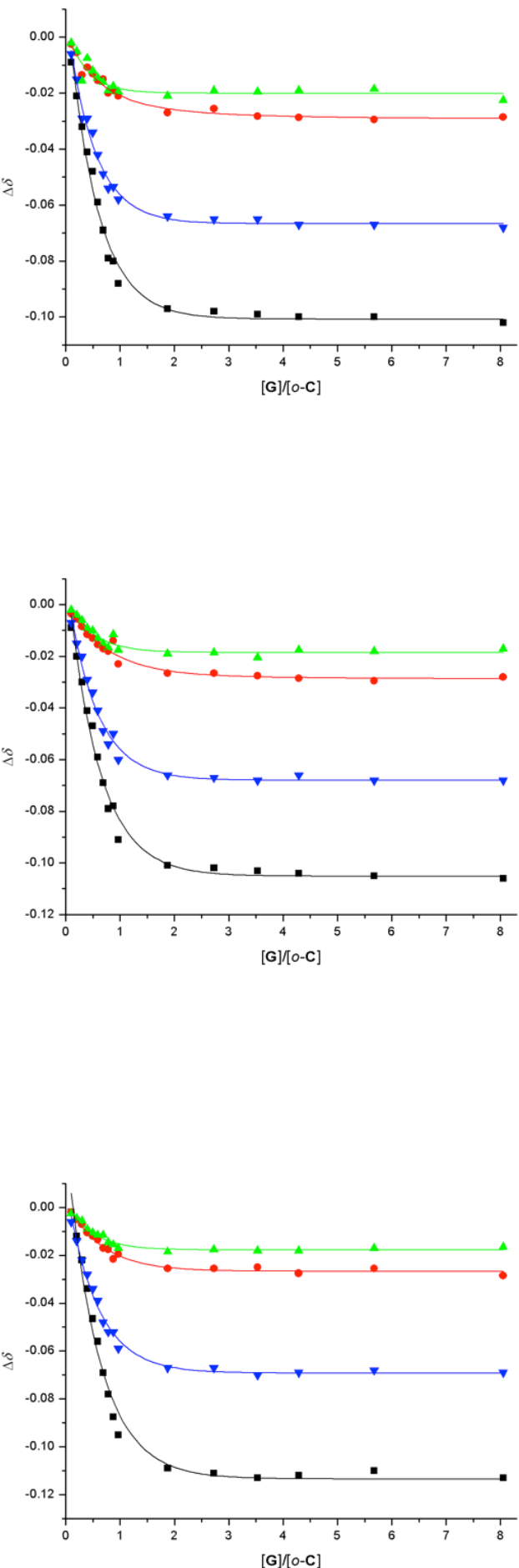

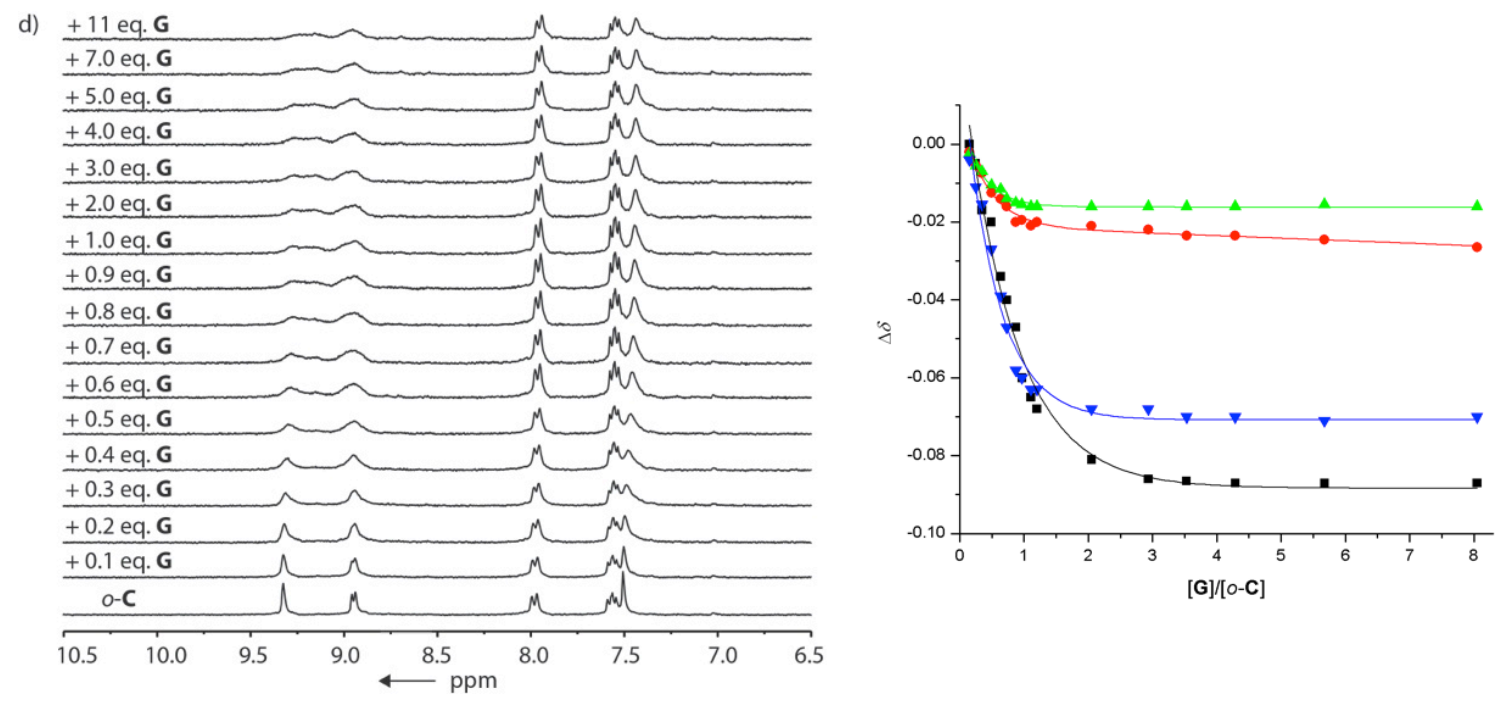

Figure 5-24 Left: ${ }^{1} \mathrm{H}$ NMR spectra $\left(300 \mathrm{MHz}, \mathrm{CD}_{3} \mathrm{CN}\right)$ of the titration of $o-\mathbf{C}(1 \mathrm{mM})$ with $\mathbf{G}$ at a) $333 \mathrm{~K}$; b) $313 \mathrm{~K}$; c) $293 \mathrm{~K}$; d) $273 \mathrm{~K}$; right: non-linear fitted binding isotherms for protons $\mathrm{H}_{\mathrm{a}}$ (black), $\mathrm{H}_{\mathrm{c}}$ (red), $\mathrm{H}_{\mathrm{d}}$ (green) and $\mathrm{H}_{\mathrm{e}}$ (blue).
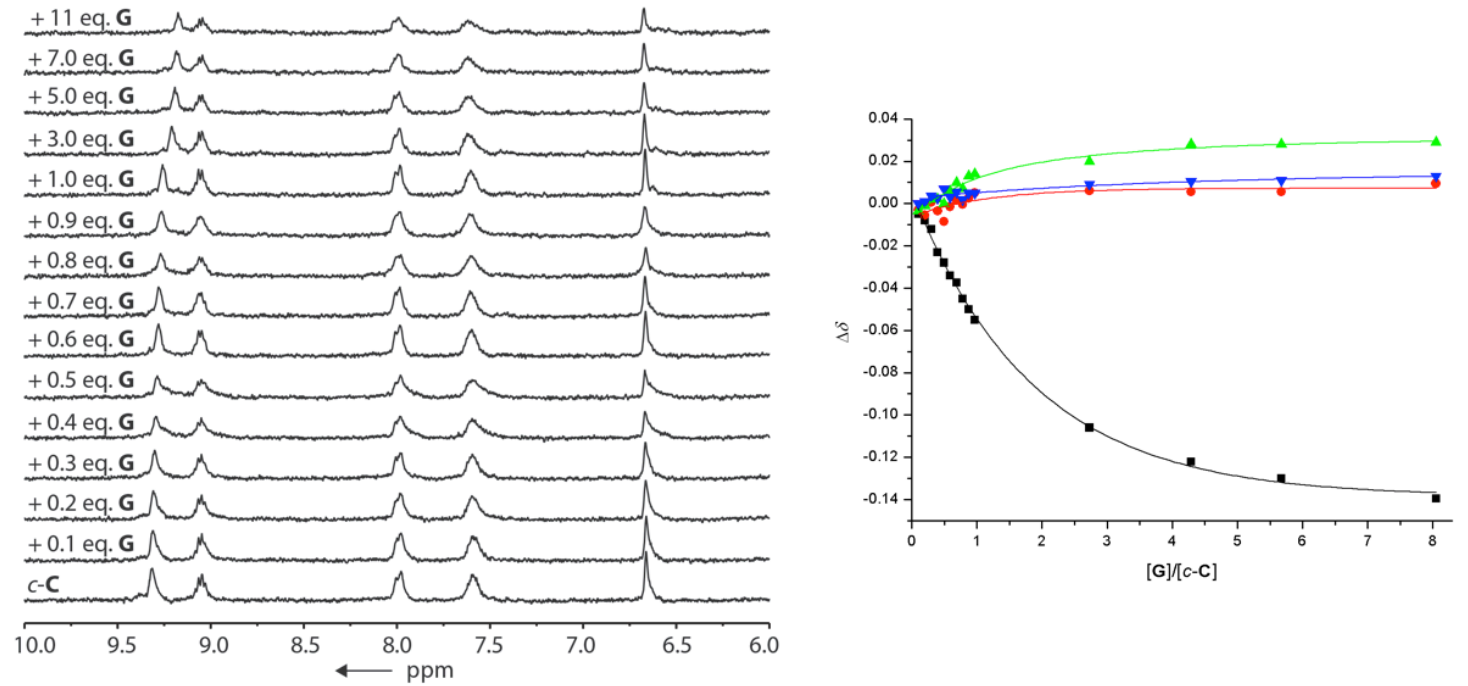

b)
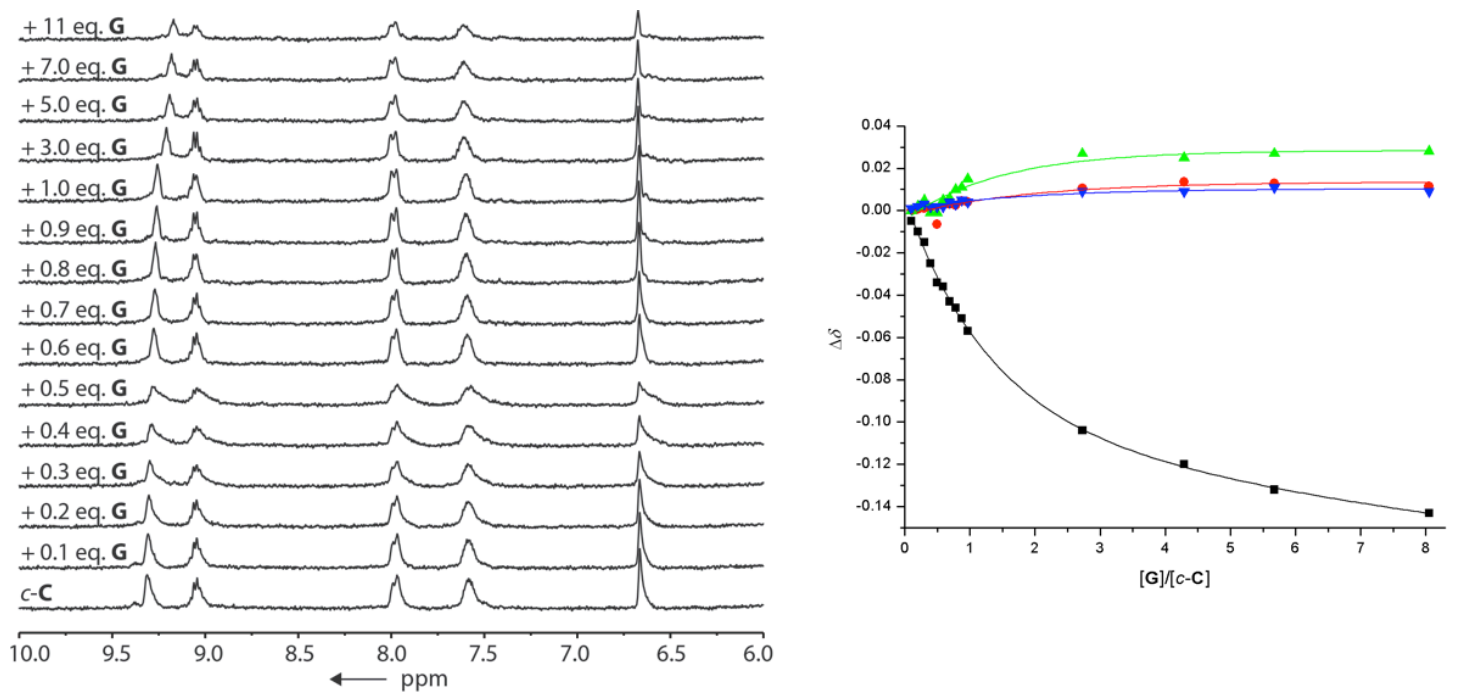

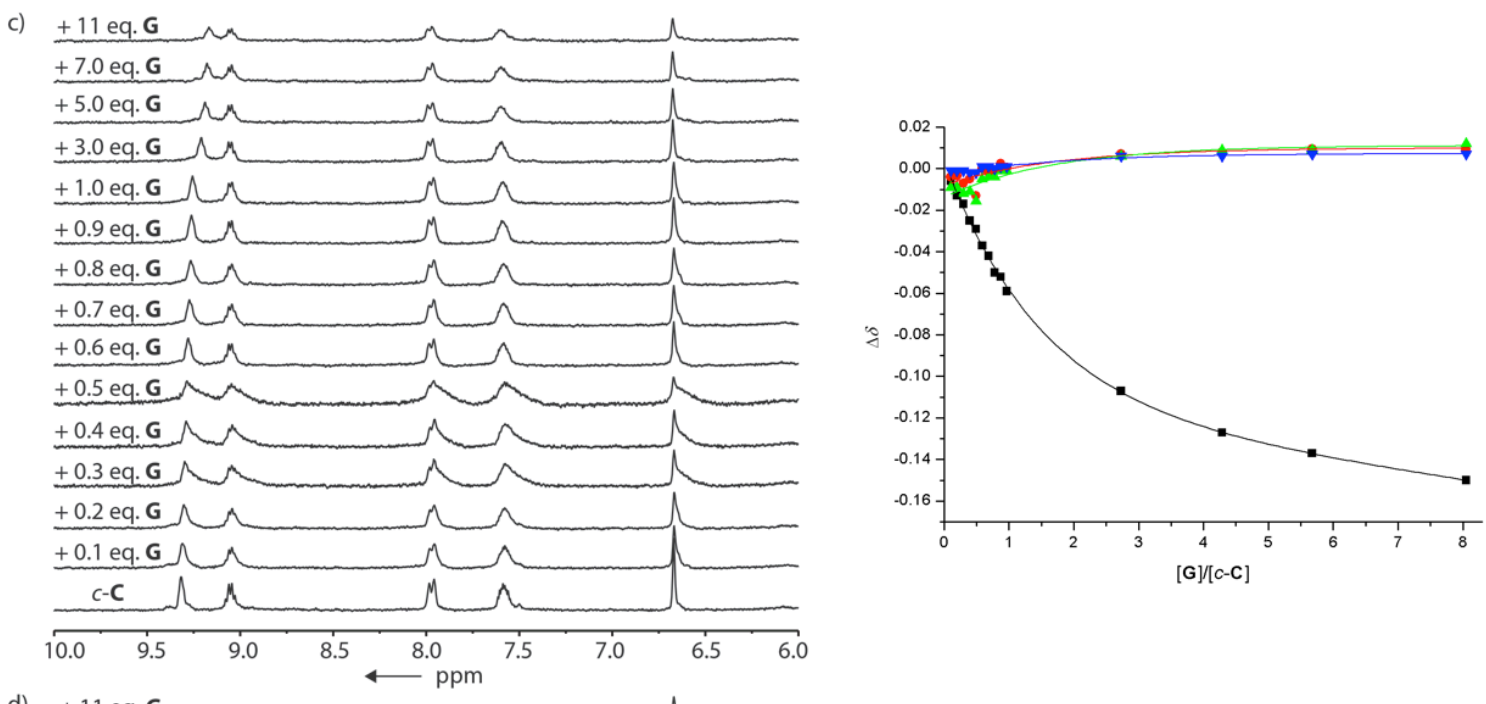

d)
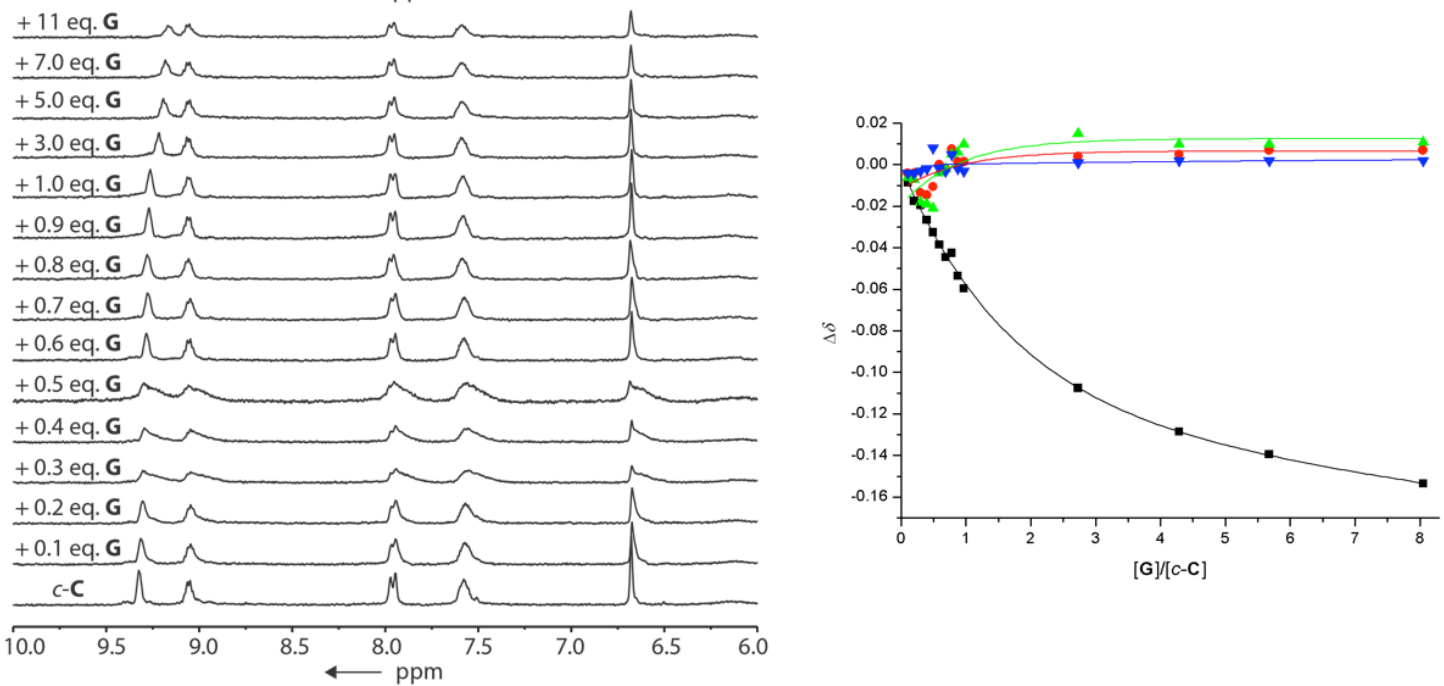

Figure 5-25 Left: ${ }^{1} \mathrm{H}$ NMR spectra $\left(300 \mathrm{MHz}, \mathrm{CD}_{3} \mathrm{CN}\right)$ of the titration of $c-\mathbf{C}(1 \mathrm{mM})$ with $\mathbf{G}$ at a) $333 \mathrm{~K}$; b) $313 \mathrm{~K}$; c) $293 \mathrm{~K}$; d) $273 \mathrm{~K}$; right: non-linear fitted binding isotherms for protons $\mathrm{H}_{\mathrm{a}}$ (black), $\mathrm{H}_{\mathrm{c}}$ (red), $\mathrm{H}_{\mathrm{d}}$ (green) and $\mathrm{H}_{\mathrm{e}}$ (blue).

Because of the structural flexibility of $o$-C, which enables the cage complex $o$-C to surround the spherical guest closely by an induced fit binding, there was a much stronger binding affinity of cage $o$-C with $\mathbf{G} K_{293, o-\mathbf{C}}=3.2 \times 10^{4} \mathrm{M}^{-1}$ than cage $c$-C with $\mathbf{G} K_{293, c-\mathbf{C}}=$ $6.7 \times 10^{2} \mathrm{M}^{-1}$ (Figure 5-26 and Table 5-3). 


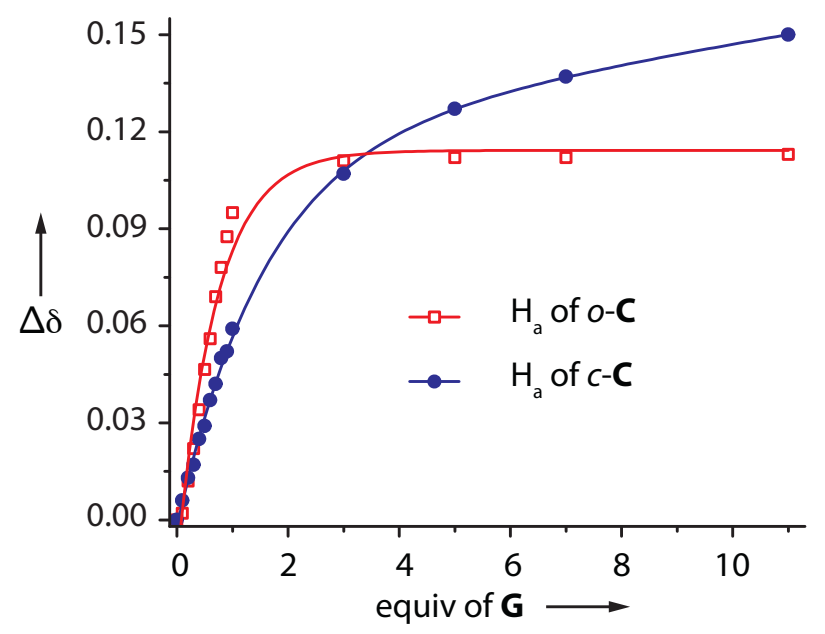

Figure 5-26 Binding isotherms at $293 \mathrm{~K}$ of the encapsulation of guest $\left[\mathrm{B}_{12} \mathrm{~F}_{12}\right]^{2-}(\mathbf{G})$ by $o-\mathrm{C}$ and $c$-C.

Table 5-3 Calculated binding constants for different temperatures.

\begin{tabular}{|c|c|c|c|c|c|c|}
\hline \multirow[b]{2}{*}{$\mathrm{T}(\mathrm{K})$} & \multicolumn{3}{|c|}{ G@o-C } & \multicolumn{3}{|c|}{$\mathbf{G} @ c-\mathbf{C}$} \\
\hline & $K_{a}\left(\mathrm{M}^{-1}\right)$ & $\begin{array}{c}\text { Standard error } \\
\text { of y-estimate } \\
(\mathrm{SEy})^{[71]}\end{array}$ & $\ln K_{a}$ & $K_{a}\left(\mathrm{M}^{-1}\right)$ & $\begin{array}{c}\text { Standard error } \\
\text { of y-estimate } \\
(\mathrm{SEy})^{[71]}\end{array}$ & $\ln K_{a}$ \\
\hline 333 & 95215.5 & $2.6 \times 10^{-3}$ & 11.46 & 673.86 & $3.0 \times 10^{-3}$ & 6.51 \\
\hline 313 & 50471.7 & $2.1 \times 10^{-3}$ & 10.83 & 746.14 & $2.5 \times 10^{-3}$ & 6.61 \\
\hline 293 & 32360.0 & $2.8 \times 10^{-3}$ & 10.38 & 671.38 & $5.1 \times 10^{-3}$ & 6.51 \\
\hline 273 & 7823.03 & $4.8 \times 10^{-3}$ & 8.96 & 665.27 & $6.8 \times 10^{-3}$ & 6.50 \\
\hline
\end{tabular}

Table 5-4 Calculated thermodynamic parameters for the encapsulation of $\mathbf{G}$ by $\boldsymbol{o}-\mathbf{C}$ and $c$-C (standard errors of the linear fit are given).

\begin{tabular}{|c|c|c|c|}
\hline \multicolumn{2}{|c|}{$\mathrm{G} @ o-\mathrm{C}$} & \multicolumn{2}{c|}{$\mathrm{G} @ c-\mathrm{C}$} \\
\hline$\Delta H^{\circ}\left(\mathrm{kJ} \mathrm{mol}^{-1}\right)$ & $\Delta S^{\circ}\left(\mathrm{J} \mathrm{K}^{-1} \mathrm{~mol}^{-1}\right)$ & $\Delta H^{\circ}\left(\mathrm{kJ} \mathrm{mol}^{-1}\right)$ & $\Delta S^{\circ}\left(\mathrm{J} \mathrm{K}^{-1} \mathrm{~mol}^{-1}\right)$ \\
\hline $30.37 \pm 4.60$ & $187.29 \pm 15.29$ & $0.60 \pm 1.03$ & $56.31 \pm 3.44$ \\
\hline
\end{tabular}

The calculated binding constant $K_{a}$ data can be plotted on a graph with $\ln K_{a}$ on the $y$-axis and $1 / T$ on the $x$-axis. And the linear relationship of the data can be found by fitting them using the linear form of the Van't Hoff equation (Figure 5-27 and Table 5-4). The encapsulation process of both $\mathbf{G} @ o-\mathbf{C}$ and $\mathbf{G} @ c-\mathbf{C}$ is obviously entropy-driven $\left(\Delta S^{\circ}{ }_{o-\mathrm{C}}=\right.$ 
$\left.187 \mathrm{~J} \mathrm{~K}^{-1} \mathrm{~mol}^{-1}, \Delta S_{c-\mathrm{C}}^{\circ}=56 \mathrm{~J} \mathrm{~K}^{-1} \mathrm{~mol}^{-1}\right)$ and endothermic $\left(\Delta H^{\circ}{ }_{o-\mathrm{C}}=30 \mathrm{~kJ} \mathrm{~mol}^{-1}, \Delta H^{\circ}{ }_{c-\mathrm{C}}=\right.$ $0.6 \mathrm{~kJ} \mathrm{~mol}^{-1}$ ), which must be a consequence of the release of solvent molecules from the interior of the cages by the large spherical guest molecule of the encapsulation process.

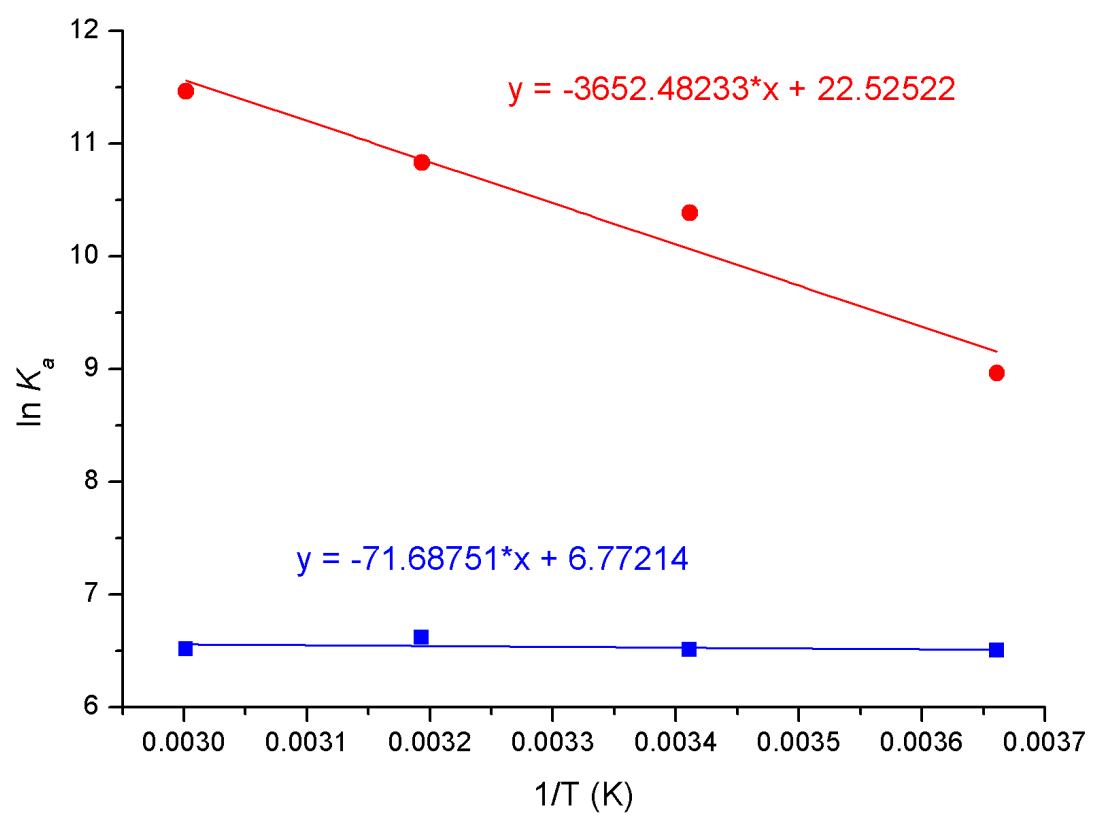

Figure 5-27 Van't Hoff plot analysis of the equilibria described by Equation 4-1 (red) and Equation 4-2 (blue).

\subsection{Conclusion}

In summary, photochromic coordination cage based on photoswitchable backbones was introduced, which can be quantitatively interconverted between a structurally flexible form $o-\mathbf{C}$ and a rigid form $c$ - $\mathbf{C}$ by altering the wavelengths of irradiation. The strongly distinguishable binding affinity for anionic guests of switched and unswitched cages has great potential to be applied in drug delivery (e.g. for boron neutron capture therapy, BNCT),[72] supramolecular catalysis, and functional constructs based on the control by external stimuli such as switchable receptors and molecular machines.[77] 


\subsection{Experimental section}

\subsubsection{Synthesis of the cage compounds}
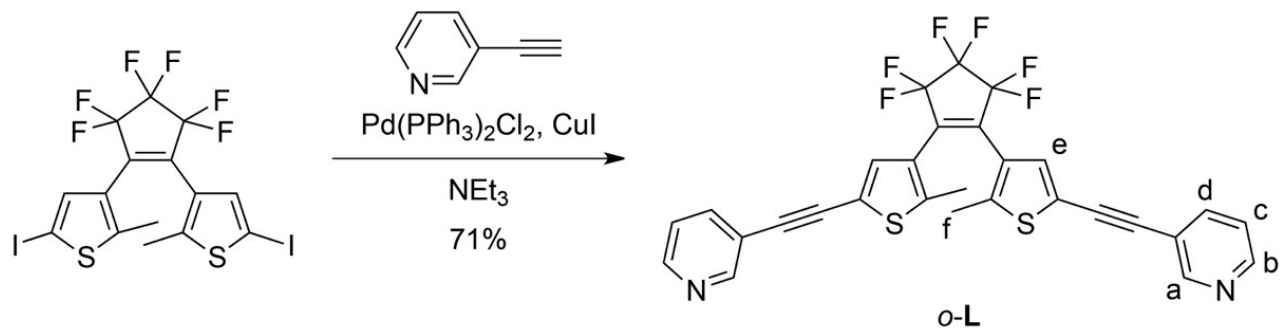

An oven dried Schlenk tube was charged with perfluoro-1,2-bis(2-iodo-5-methylthien-4yl)cyclopentene ${ }^{[67,68]}$ (300 mg, $0.51 \mathrm{mmol}$ ), 3-ethynylpyridine (158.9 mg, $1.54 \mathrm{mmol}$ ), CuI (6.84 mg, $0.036 \mathrm{mmol}), \mathrm{Pd}\left(\mathrm{PPh}_{3}\right)_{2} \mathrm{Cl}_{2}(18.0 \mathrm{mg}, 0.026 \mathrm{mmol})$ and degassed triethylamine $(9 \mathrm{~mL})$. The mixture was reflux with stirring under $\mathrm{N}_{2}$ for $12 \mathrm{~h}$ at $90^{\circ} \mathrm{C}$. Following removal of $\mathrm{NEt}_{3}, \mathrm{CHCl}_{3}$ was added and the solution was washed with water and brine, dried over anhydrous $\mathrm{MgSO}_{4}$ and concentrated in vacuo. The product was purified by column chromatography on silica gel using $\mathrm{CHCl}_{3} / \mathrm{CH}_{3} \mathrm{OH}(100: 1)$ as eluents and isolated $o$-L (206 $\mathrm{mg}, 0.36 \mathrm{mmol}$ ) as a bluish solid in $71 \%$ yield.

1H-NMR (300 MHz, CD $\left.{ }_{3} \mathrm{CN}\right): \delta 2.01\left(6 \mathrm{H}, \mathrm{s}, \mathrm{H}_{\mathrm{f}}\right), 7.35\left(2 \mathrm{H}, \mathrm{s}, \mathrm{H}_{\mathrm{e}}\right), 7.38\left(2 \mathrm{H}, \mathrm{dd},{ }^{3} \mathrm{~J}=7.9 \mathrm{~Hz}, 3 \mathrm{~J}=\right.$ $\left.4.9 \mathrm{~Hz}, \mathrm{H}_{\mathrm{c}}\right), 7.86\left(2 \mathrm{H}, \mathrm{ddd},{ }^{3} \mathrm{~J}=7.9 \mathrm{~Hz},{ }^{4} \mathrm{~J}=2.2 \mathrm{~Hz}, 1.7 \mathrm{~Hz}, \mathrm{H}_{\mathrm{d}}\right), 8.56\left(2 \mathrm{H}, \mathrm{dd},{ }^{3} \mathrm{~J}=4.9 \mathrm{~Hz},{ }^{4} \mathrm{~J}=\right.$ $\left.1.7 \mathrm{~Hz}, \mathrm{H}_{\mathrm{b}}\right), 8.71\left(2 \mathrm{H}, \mathrm{d}, 4 J=2.2 \mathrm{~Hz}, \mathrm{H}_{\mathrm{a}}\right)$.

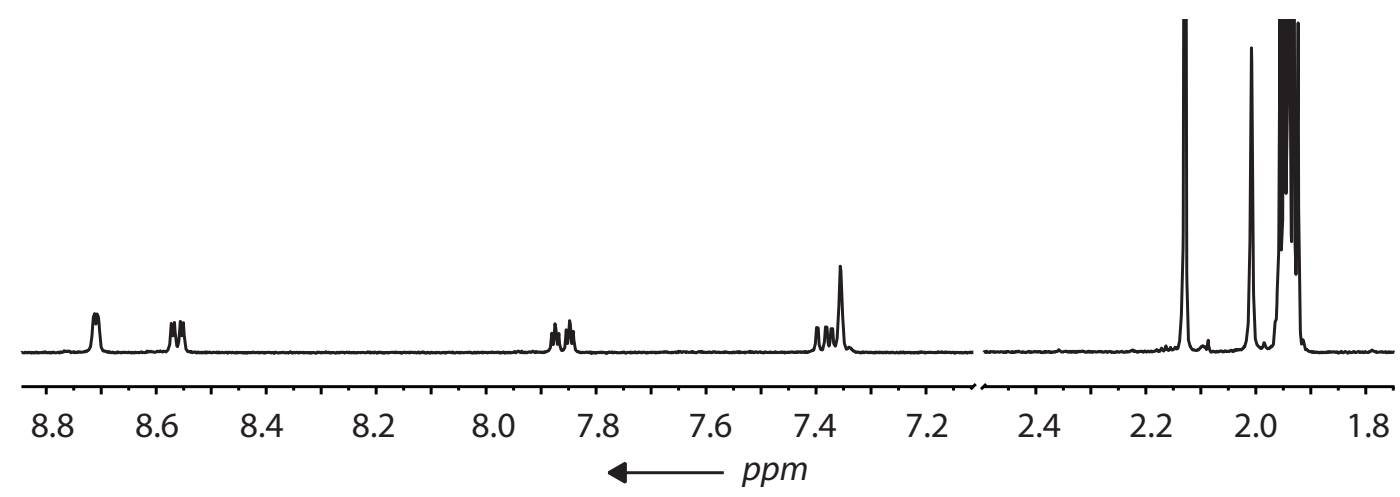

${ }^{13} \mathrm{C}-\mathrm{NMR}\left(75 \mathrm{MHz}, \mathrm{CD}_{3} \mathrm{CN}\right): \delta 14.73,84.81,91.41,112.03\left(\underline{\mathrm{CF}}_{2}\right), 116.97\left(\underline{\mathrm{CF}}_{2}\right), 120.22$, $121.82,124.35,125.50,133.12,137.37,139.17,146.02,150.21,152.64$ ( $\underline{C F}_{2}$ signals could be detected in the ${ }^{19} \mathrm{~F}$-decoupled ${ }^{13} \mathrm{C}$ spectrum, see inset in green). 


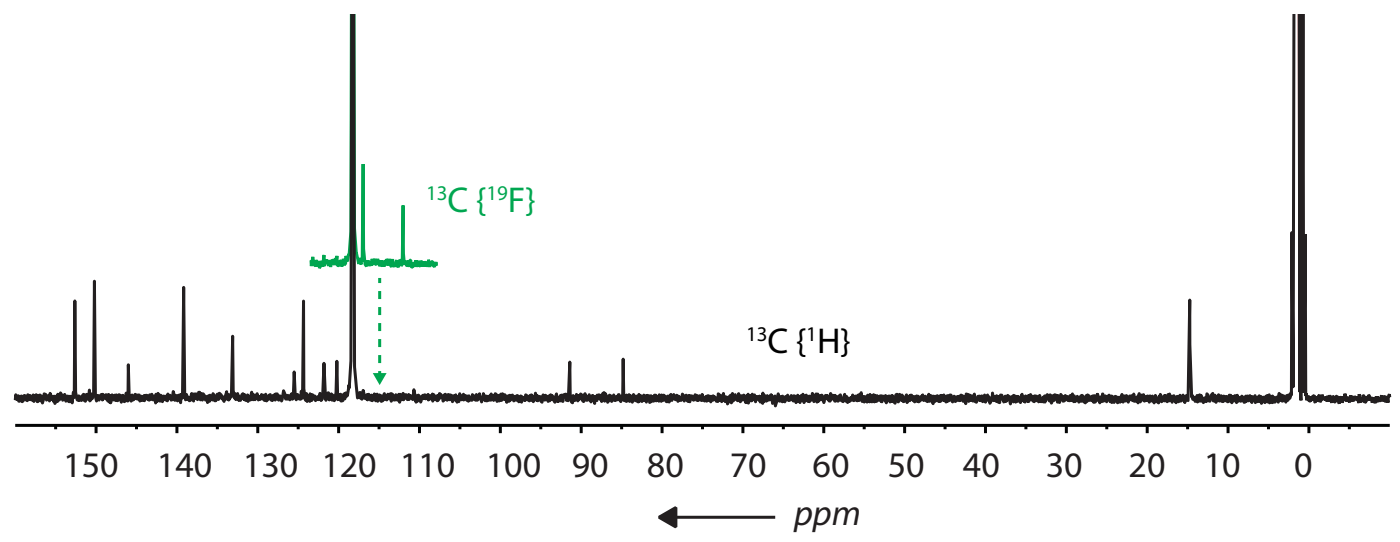

19F-NMR (282 MHz, $\left.\mathrm{CD}_{3} \mathrm{CN}\right): \delta-110.86(4 \mathrm{~F}, \mathrm{t}, 3 \mathrm{~J}=5.0 \mathrm{~Hz}),-132.41\left(2 \mathrm{~F}\right.$, quint, $\left.{ }^{3} J=5.2 \mathrm{~Hz}\right)$.

MS (ESI, pos.) $m / z=571.1[o-\mathbf{L}+\mathrm{H}]^{+}, 593.1[o-\mathbf{L}+\mathrm{Na}]^{+}$.

IR (ATR): 397, 524, 577, 631, 703, 739, 804, 858, 1022, 1047, 1121, 1192, 1275, 1338, $1406,1438,1485,1560,2211,3037 \mathrm{~cm}^{-1}$.

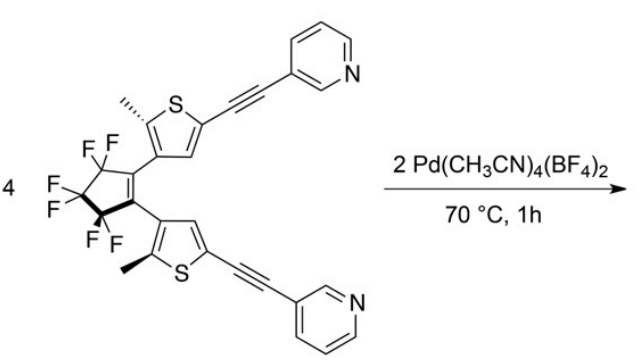

O-L

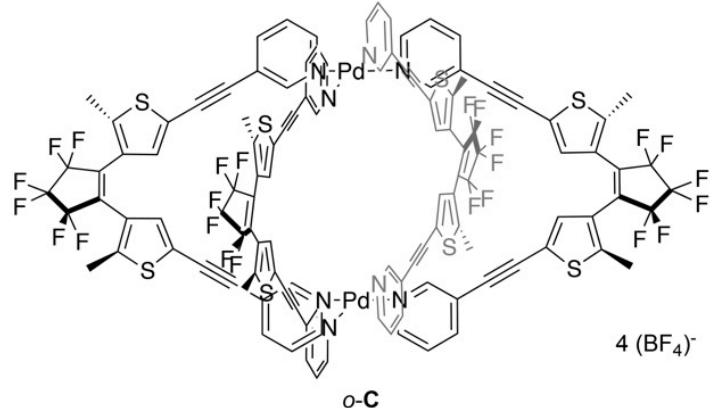

O-C

The open cage compound was synthesized by heating a mixture of the ligand $o$-L $(12 \mu \mathrm{mol}$, $400 \mu \mathrm{L}$ of a $30 \mathrm{mM}$ stock solution in $\left.\mathrm{CD}_{3} \mathrm{CN}\right)$ and $\mathrm{Pd}\left(\mathrm{CH}_{3} \mathrm{CN}\right)_{4}\left(\mathrm{BF}_{4}\right)_{2}(6 \mu \mathrm{mol}, 200 \mu \mathrm{L}$ of a $30 \mathrm{mM}$ stock solution in $\mathrm{CD}_{3} \mathrm{CN}$ ) in $2400 \mu \mathrm{L} \mathrm{CD}_{3} \mathrm{CN}$ at $70{ }^{\circ} \mathrm{C}$ for $1 \mathrm{~h}$ in a closed vial to yield $3000 \mu \mathrm{L}$ of a $1 \mathrm{mM}$ solution of the cage compound $o-\mathrm{C}$ in quantitative yield.

1H-NMR (300 MHz, CD $\left.{ }_{3} \mathrm{CN}\right): \delta 1.96\left(24 \mathrm{H}, \mathrm{s}, \mathrm{H}_{\mathrm{f}}\right), 7.51\left(8 \mathrm{H}, \mathrm{s}, \mathrm{H}_{\mathrm{e}}\right), 7.58\left(8 \mathrm{H}, \mathrm{dd},{ }^{3} \mathrm{~J}=8.1 \mathrm{~Hz},{ }^{3} \mathrm{~J}\right.$ $\left.=5.8 \mathrm{~Hz}, \mathrm{H}_{\mathrm{c}}\right), 8.00\left(8 \mathrm{H}, \mathrm{dt},{ }^{3} \mathrm{~J}=8.1 \mathrm{~Hz},{ }^{4} \mathrm{~J}=1.6 \mathrm{~Hz}, \mathrm{H}_{\mathrm{d}}\right), 8.96\left(8 \mathrm{H}, \mathrm{d}, 3 J=5.8 \mathrm{~Hz}, \mathrm{H}_{\mathrm{b}}\right), 9.34(8 \mathrm{H}$, d, $\left.4 J=1.8 \mathrm{~Hz}, \mathrm{H}_{\mathrm{a}}\right)$.

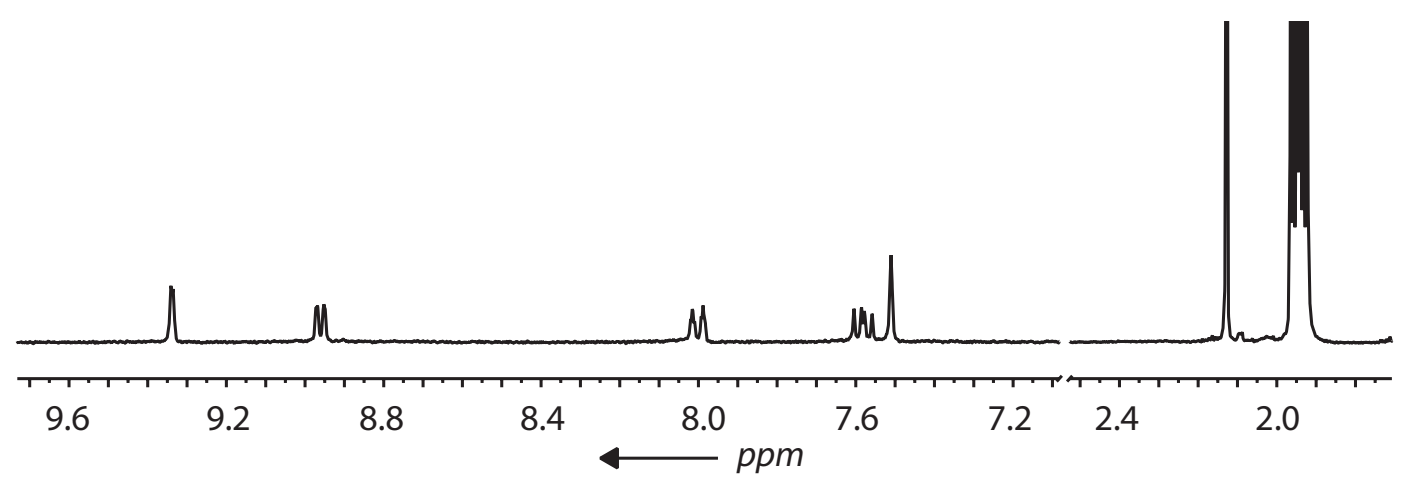


13C-NMR (125 MHz, CD $\left.{ }_{3} \mathrm{CN}, 300 \mathrm{~K}\right): \delta 15.05,87.96,89.05,121.10,124.39,125.74,128.25$, $133.24,142.84,147.34,151.01,154.24$ (Signals of perfluorocyclopentene ring not detected).

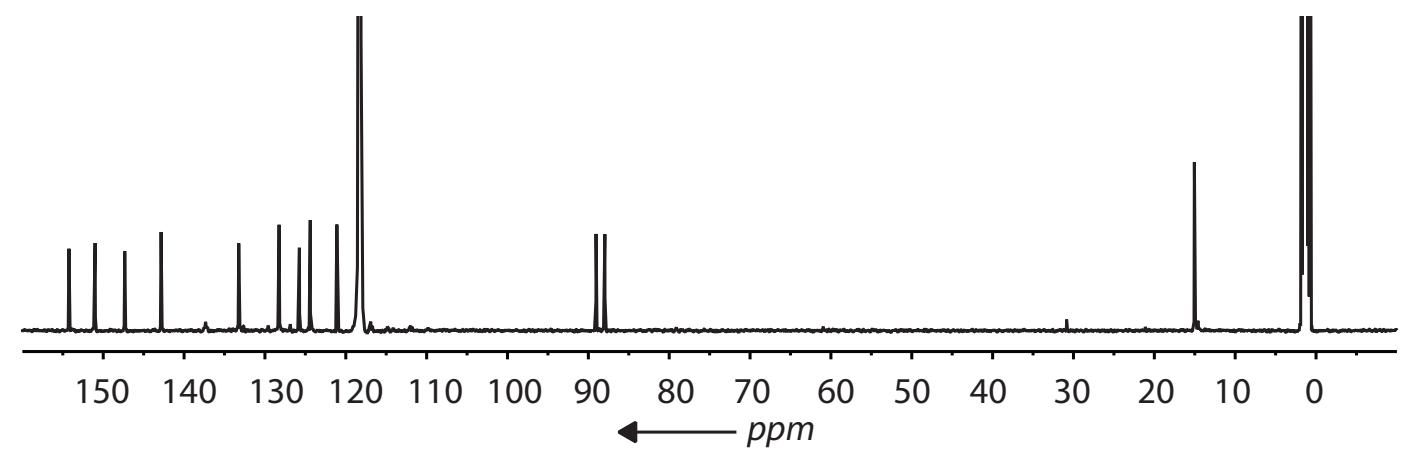

19F-NMR (282 MHz, CD $\left.{ }_{3} \mathrm{CN}\right): \delta-111.17(16 \mathrm{~F}, \mathrm{~m}),-132.14(8 \mathrm{~F}, \mathrm{~m}),-150.82\left(16 \mathrm{~F}, \mathrm{~s}, \mathrm{BF}_{4}^{-}\right)$.

MS (ESI, pos.) $m / z=623.8[o-\mathrm{C}]^{4+}, 860.7\left[o-\mathbf{C}+\mathrm{BF}_{4}\right]^{3+}, 1334.0\left[o-\mathbf{C}+2 \mathrm{BF}_{4}\right]^{2+}$

HRMS (ESI, pos.) $m / z\left(\left[o-C+B_{4}\right]^{3+}\right)$ calcd. $=860.3587$, found $=860.3581$.

IR (ATR): 398, 525, 576, 630, 695, 740, 816, 901, 984, 1043, 1194, 1275, 1339, 1421, 1489, $1574,1662,2214,3086 \mathrm{~cm}^{-1}$.

The closed cage compound was synthesized in quantitative yield by heating a mixture of the ligand $c$ - $\mathbf{L}\left(12 \mu \mathrm{mol}, 400 \mu \mathrm{L}\right.$ of a $30 \mathrm{mM}$ stock solution in $\left.\mathrm{CD}_{3} \mathrm{CN}\right)$ and $\mathrm{Pd}\left(\mathrm{CH}_{3} \mathrm{CN}\right)_{4}\left(\mathrm{BF}_{4}\right)_{2}$ ( $6 \mu \mathrm{mol}, 200 \mu \mathrm{L}$ of a $30 \mathrm{mM}$ stock solution in $\mathrm{CD}_{3} \mathrm{CN}$ ) in $2400 \mu \mathrm{L} \mathrm{CD}_{3} \mathrm{CN}$ at $70{ }^{\circ} \mathrm{C}$ for $1 \mathrm{~h}$ in a closed vial to yield $3000 \mu \mathrm{L}$ of a $1 \mathrm{mM}$ solution of the cage compound $c$-C.

1H-NMR (300 MHz, CD 3 CN): $\delta 2.28\left(24 \mathrm{H}, \mathrm{s}, \mathrm{H}_{\mathrm{f}}\right), 6.68\left(8 \mathrm{H}, \mathrm{s}, \mathrm{H}_{\mathrm{e}}\right), 7.60\left(8 \mathrm{H}, \mathrm{m}, \mathrm{H}_{\mathrm{c}}\right), 7.99(8 \mathrm{H}$, $\left.\mathrm{m}, \mathrm{H}_{\mathrm{d}}\right), 9.06\left(8 \mathrm{H}, \mathrm{m}, \mathrm{H}_{\mathrm{b}}\right), 9.33\left(\mathrm{~m}, 8 \mathrm{H}, \mathrm{H}_{\mathrm{a}}\right)$.

\subsubsection{Temperature dependent titrations of $o-\mathbf{C}$ and $c$ - $\mathbf{C}$ with $\left[\mathrm{B}_{12} \mathrm{~F}_{12}\right]^{2-}$ guest}

The guest compound was synthesized according to the literature[88] by adding an excess of $\left[\mathrm{NBu}_{4}\right] \mathrm{Br}$ to an aqueous solution of $\mathrm{K}_{2}\left[\mathrm{~B}_{12} \mathrm{~F}_{12}\right]$. The immediately formed white precipitation was separated by filtration and washed several times with water. After drying in vacuo the guest compound was obtained as a white powder.

1H-NMR (300 MHz, CD $\left.{ }_{3} \mathrm{CN}\right): \delta 0.97\left(24 \mathrm{H}, \mathrm{t},{ }^{3} J=7.3 \mathrm{~Hz}, \mathrm{CH}_{3}\right), 1.35(16 \mathrm{H}$, sextet, $3 J=7.3 \mathrm{~Hz}$, $\mathrm{C}_{2}-\mathrm{CH}_{3}$ ), 1.52-1.67 (16H, m, $\mathrm{CH}_{2} \mathrm{C}_{2} \mathrm{CH}_{2}$ ), 3.03-3.11 (16H, m, NCي $\underline{\mathrm{H}}_{2}$ ).

19F-NMR (282 MHz, $\left.\mathrm{CD}_{3} \mathrm{CN}\right): \delta-269.47$ (s).

11B-NMR (96 MHz, $\mathrm{CD}_{3} \mathrm{CN}$ ): $\delta-18.04$ (s). 
The host-guest complexes were prepared by titrating a stock solution of $\left(\mathrm{NBu}_{4}\right)_{2}\left[\mathrm{~B}_{12} \mathrm{~F}_{12}\right]$ $\left(30 \mathrm{mM}\right.$ in $\left.\mathrm{CD}_{3} \mathrm{CN}\right)$ stepwise into a cage solution $o-\mathrm{C}$ and $c$-C $(600 \mu \mathrm{L}, 1 \mathrm{mM}) .{ }^{1} \mathrm{H}$ NMR spectra were recorded at various temperatures (333 K, $313 \mathrm{~K}, 293 \mathrm{~K}, 273 \mathrm{~K}$ ). All the titrations of $c$-C were carried out in the dark.

\subsection{References}

[1] M. Irie, Chem. Rev. 2000, 100, 1685.

[2] H. Tian, S. Yang, Chem. Soc. Rev. 2004, 33, 85.

[3] O. Wenger, Chem. Soc. Rev. 2012, 41, 3772.

[4] K. Uchida, Y. Nakayama, M. Irie, Bull. Chem. Soc. Jpn. 1990, 63, 1311.

[5] S. Nakamura, M. Irie, J. Org. Chem. 1988, 53, 6136.

[6] J. Chauvin, T. Kawai, M. Irie, Jpn, J. Appl. Phys. 2001, 40, 2518.

[7] A. Rajbanshi, B. A. Moyer, R. Custelcean, Cryst. Growth Des. 2011, 11, 2702.

[8] W. Meng, B. Breiner, K. Rissanen, J. D. Thoburn, J. K. Clegg, J. R. Nitschke, Angew. Chem. Int. Ed. 2011, 50, 3479.

[9] D. Cram, M. Tanner, R. Thomas, Angew. Chem. Int. Ed. 1991, 30, 1024.

[10] K. Goto, R. Okazaki, Liebigs Ann./Recueil 1997, 2393.

[11] M. Ziegler, J. Brumaghim, K. Raymond, Angew. Chem. Int. Ed. 2000, 39, 4119.

[12] D. Fiedler, R. G. Bergman, K. N. Raymond, Angew. Chem. Int. Ed. 2006, 45, 745.

[13] M. Kawano, Y. Kobayashi, T. Ozeki, M. Fujita, J. Am. Chem. Soc. 2006, 128, 6558.

[14] F. Hapiot, S. Tilloy, E. Monflier, Chem. Rev. 2006, 106, 767.

[15] T. Iwasawa, R. J. Hooley, J. Rebek, Science 2007, 317, 493.

[16] R. J. Hooley, J. Rebek, Chem. Biol. 2009, 16, 255.

[17] C.-Y. Gao, L. Zhao, M.-X. Wang, J. Am. Chem. Soc. 2012, 134, 824.

[18] J. L. Sessler, P. Gale, W.-S. Cho, S. J. Rowan, Anion Receptor Chemistry (Monographs in Supramolecular Chemistry), Royal Society of Chemistry, 2006.

[19] R. Custelcean, J. Bosano, P. V. Bonnesen, V. Kertesz, B. P. Hay, Angew. Chem. Int. Ed. 2009, 48, 4025 .

[20] S. O. Kang, J. M. Llinares, V. W. Day, K. Bowman-James, Chem. Soc. Rev. 2010, 39, 3980.

[21] M. Wang, V. Vajpayee, S. Shanmugaraju, Y.-R. Zheng, Z. Zhao, H. Kim, P. S. Mukherjee, K.-W. Chi, P. J. Stang, Inorg. Chem. 2011, 50, 1506.

[22] J. A. Thomas, Dalton Trans. 2011, 12005.

[23] P. Mal, B. Breiner, K. Rissanen, J. R. Nitschke, Science 2009, 324, 1697.

[24] I. A. Riddell, M. M. J. Smulders, J. K. Clegg, J. R. Nitschke, Chem. Commun. 2011, 457.

[25] T. D. Nguyen, Y. Liu, S. Saha, K. C. F. Leung, J. F. Stoddart, J. I. Zink, J. Am. Chem. Soc. 2007, 129, 626.

[26] P. Mal, D. Schultz, K. Beyeh, K. Rissanen, J. R. Nitschke, Angew. Chem. Int. Ed. 2008, 47, 8297. 
[27] F. Schmitt, J. Freudenreich, N. P. E. Barry, L. Juillerat-Jeanneret, G. Süss-Fink, B. Therrien, J. Am. Chem. Soc. 2011, 134, 754.

[28] Z. Ma, B. Moulton, Coord. Chem. Rev. 2011, 255, 1623.

[29] J. E. M. Lewis, E. L. Gavey, S. A. Cameron, J. D. Crowley, Chem. Sci. 2012, 3, 778.

[30] D. Vriezema, M. Aragones, J. Elemans, J. Cornelissen, A. Rowan, R. Nolte, Chem. Rev. 2005, 105, 1445.

[31] M. Yoshizawa, J. K. Klosterman, M. Fujita, Angew. Chem. Int. Ed. 2009, 48, 3418.

[32] U. H. Brinker, J. Mieusset (Eds.), Molecular Encapsulation: Organic Reactions in Constrained Systems, Wiley, 2010.

[33] M. J. Wiester, P. A. Ulmann, C. A. Mirkin, Angew. Chem. Int. Ed. 2011, 50, 114.

For one resort from this dilemma see $[28,29]$ :

[34] Fiedler, D.; van Halbeek, H.; Bergman, R. G.; Raymond, K. N. J. Am. Chem. Soc. 2006, $128,10240$.

[35] C. J. Hastings, M. D. Pluth, R. G. Bergman, K. N. Raymond, J. Am. Chem. Soc. 2010, 132, 6938.

For other approaches towards light-triggered catalysis see [30-33]:

[36] D. Sud, T. B. Norsten, N. R. Branda, Angew. Chem. Int. Ed. 2005, 44, 2019;

[37] R. S. Stoll, S. Hecht, Angew. Chem. Int. Ed. 2010, 49, 5054;

[38] J. Wang, B. L. Feringa, Science 2011, 331, 1429;

[39] U. Lüning, Angew. Chem. Int. Ed. 2012, 51, 8163.

[40] G. H. Clever, S. Tashiro, M. Shionoya, J. Am. Chem. Soc. 2010, 132, 9973.

[41] H. Dube, D. Ajami, J. Rebek, Angew. Chem. Int. Ed. 2010, 49, 3192;

[42] H. Dube, J. Rebek, Angew. Chem. Int. Ed. 2012, 51, 3207.

[43] M. R. Banghart, A. Mourot, D. L. Fortin, J. Z. Yao, R. H. Kramer, D. Trauner, Angew. Chem. Int. Ed. 2009, 48, 9097.

[44] G. H. Clever, "Switchable host-guest interactions of supramolecular rings and cages", Molecules at Work (Ed.: B. Pignataro), Wiley-VCH, 2012.

[45] S. Shinkai, M. Ishihara, K. Ueda, O. Manabe, J. Incl. Phenom. Macro. 1984, 2, 111.

[46] M. Irie, M. Kato, J. Am. Chem. Soc. 1985, 107, 1024.

[47] M. Blank, L. Soo, N. Wassermann, B. Erlanger, Science 1981, 214, 70.

[48] A. Ueno, H. Yoshimura, R. Saka, T. Osa, J. Am. Chem. Soc. 1979, 101, 2779.

[49] R. Rojanathanes, T. Tuntulani, W. Bhanthumnavin, M. Sukwattanasinitt, Org. Lett. 2005, 7, 3401.

[50] H. Kai, S. Nara, K. Kinbara, T. Aida, J. Am. Chem. Soc. 2008, 130, 6725.

[51] R. Reuter, N. Hostettler, M. Neuburger, H. Wegner, Eur. J. Org. Chem. 2009, 5647.

[52] N. Iwasawa, H. Takahagi, K. Ono, K. Fujii, H. Uekusa, Chem. Commun. 2012, 7477.

[53] S. Chen, L.-J. Chen, H.-B. Yang, H. Tian, W. Zhu, J. Am. Chem. Soc. 2012, 134, 13596.

Covalently attached azobenzenes were used for switching the interior hydrophobicity (but not structure) of a coordination cage:

[54] T. Murase, S. Sato, M. Fujita, Angew. Chem. Int. Ed. 2007, 46, 5133. 
[55] M. Fujita, K. Umemoto, M. Yoshizawa, N. Fujita, T. Kusukawa, K. Biradha, Chem. Commun. 2001, 509.

[56] S. J. Dalgarno, N. P. Power, J. L. Atwood, Coord. Chem. Rev. 2008, 252, 825.

[57] D. Tranchemontagne, Z. Ni, M. O'Keeffe, O. Yaghi, Angew. Chem. Int. Ed. 2008, 47, 5136.

Several other examples of coordination cages obeying the general formula $\left[\mathrm{M}_{2} \mathrm{~L}_{4}\right]^{4+}$ where $\mathrm{M}$ is a square-planar coordinated metal ion such as $\mathrm{Pd}(\mathrm{II})$ or $\mathrm{Pt}(\mathrm{II})$ and $\mathrm{L}$ are bis-pyridyl ligands bridging the two metal centers have been reported. See for example [52-61]:

[58] D. A. McMorran, P. J. Steel, Angew. Chem. Int. Ed. 1998, 37, 3295.

[59] D. K. Chand, K. Biradha, M. Fujita, Chem. Commun. 2001, 1652.

[60] C. Su, Y. P. Cai, C. Chen, M. D. Smith, W. Kaim, H. C. zur Loye, J. Am. Chem. Soc. 2003, $125,8595$.

[61] N. L. S. Yue, D. J. Eisler, M. C. Jennings, R. J. Puddephatt, Inorg. Chem. 2004, 43, 7671.

[62] G. H. Clever, S. Tashiro, M. Shionoya, Angew. Chem. Int. Ed. 2009, 48, 7010.

[63] P. Liao, B. W. Langloss, A. M. Johnson, E. R. Knudsen, F. S. Tham, R. R. Julian, R. J. Hooley, Chem. Commun. 2010, 4932.

[64] N. Kishi, Z. Li, K. Yoza, M. Akita, M. Yoshizawa, J. Am. Chem. Soc. 2011, 133, 11438.

[65] G. H. Clever, W. Kawamura, S. Tashiro, M. Shiro, M. Shionoya, Angew. Chem. Int. Ed. 2012, 51, 2606.

[66] S. Freye, J. Hey, A. Torras Galán, D. Stalke, R. Herbst Irmer, M. John, G. H. Clever, Angew. Chem. Int. Ed. 2012, 51, 2191.

[66] D. M. Engelhard, S. Freye, K. Grohe, M. John, G. H. Clever, Angew. Chem. Int. Ed. 2012, $51,4747$.

[67] S. Fraysse, C. Coudret, J. P. Launay, Eur. J. Inorg. Chem. 2000, 1581.

[68] B. He, O. S. Wenger, J. Am. Chem. Soc. 2011, 133, 17027.

[69] Y. Ishibashi, M. Fujiwara, T. Umesato, H. Saito, S. Kobatake, M. Irie, H. Miyasaka, J. Phys. Chem. C 2011, 115, 4265.

[70] G. H. Clever, M. Shionoya, Chem. Eur. J. 2010, 16, 11792.

[71] T. Weilandt, U. Kiehne, J. Bunzen, G. Schnakenburg, A. Lützen, Chem. Eu. J. 2010, 16, 2418.

[72] N. Ousaka, J. K. Clegg; J. R. Nitschke, Angew. Chem. Int. Ed. 2012, 51, 1464.

[73] M. Han, J. Hey, W. Kawamura, D. Stalke, M. Shionoya, G. H. Clever, Inorg. Chem. 2012, $51,9574$.

[74] G. H. Clever, W. Kawamura, M. Shionoya, Inorg. Chem. 2011, 50, 4689.

[75] P. Thordarson, Chem. Soc. Rev. 2011, 40, 1305.

[76] R. F. Barth, J. A. Coderre, M. Vicente, T. E. Blue, Clin. Cancer Res. 2005, 11, 3987.

[77] E. R. Kay, D. A. Leigh, F. Zerbetto, Angew. Chem. Int. Ed. 2007, 46, 72.

[78] Spartan 08 Software, Wavefunction Inc., Irvine CA, 2008.

[79] Gaussian 09, M. J. Frisch, G. W. Trucks, H. B. Schlegel, G. E. Scuseria, M. A. Robb, J. R. Cheeseman, G. Scalmani, V. Barone, B. Mennucci, G. A. Petersson, H. Nakatsuji, M. Caricato, X. Li, H. P. Hratchian, A. F. Izmaylov, J. Bloino, G. Zheng, J. L. Sonnenberg, M. Hada, M. Ehara, K. Toyota, R. Fukuda, J. Hasegawa, M. Ishida, T. Nakajima, Y. Honda, 
O. Kitao, H. Nakai, T. Vreven, J. A. Montgomery, Jr., J. E. Peralta, F. Ogliaro, M. Bearpark, J. J. Heyd, E. Brothers, K. N. Kudin, V. N. Staroverov, R. Kobayashi, J. Normand, K. Raghavachari, A. Rendell, J. C. Burant, S. S. Iyengar, J. Tomasi, M. Cossi, N. Rega, J. M. Millam, M. Klene, J. E. Knox, J. B. Cross, V. Bakken, C. Adamo, J. Jaramillo, R. Gomperts, R. E. Stratmann, O. Yazyev, A. J. Austin, R. Cammi, C. Pomelli, J. W. Ochterski, R. L. Martin, K. Morokuma, V. G. Zakrzewski, G. A. Voth, P. Salvador, J. J. Dannenberg, S. Dapprich, A. D. Daniels, O. Farkas, J. B. Foresman, J. V. Ortiz, J. Cioslowski and D. J. Fox, Gaussian, Inc., Wallingford CT, 2009.

[80] T. Kottke, D. Stalke, J. Appl. Crystallogr. 1993, 26, 615.

[81] T. Kottke, R. J. Lagow, D. Stalke, J. Appl. Crystallogr. 1996, 29, 465.

[82] D. Stalke, Chem. Soc. Rev. 1998, 27, 171.

[83] Bruker, SAINT v7.68A, Madison, WI, USA, 2009.

[84] G. M. Sheldrick, SADABS 20012/1, Göttingen, 2012.

[85] G. M. Sheldrick, Acta Crystallogr., Sect. A. 2008, 64, 112.

[86] F. H. Allen, Acta Crystallogr. 2002, B58, 380.

[87] David R. Lide, Ed., CRC Handbook of Chemistry and Physics, 89th Edition (Internet Version 2009), CRC Press/Taylor and Francis, Boca Raton, FL.

[88] V. Geis, K. Guttsche, C. Knapp, H. Scherer and R. Uzum, Dalton Trans. 2009, 2687. 


\section{LIGHT-TRIGGERED CONVERSION BETWEEN A SELF-ASSEMBLED TRIANGLE AND SPHERE ${ }^{5}$}

In this chapter, another light-switchable ligand $\mathbf{L}^{\mathbf{2}}$ based on the same photochromic dithienylethene (DTE) backbones used in Chapter 4 was synthesized. A small $\operatorname{Pd}_{3}\left(o-\mathbf{L}^{2}\right)_{6}$ three-ring constructed from square-planar-coordinated $\mathrm{Pd}^{\mathrm{II}}$ ions and these bismonodentate ligands can be dramatically transformed into a rhombicuboctahedral $\operatorname{Pd}_{24}\left(c-\mathbf{L}^{2}\right)_{48}$ sphere with about $7 \mathrm{~nm}$ diameter upon light-irradiation. By altering the wavelength of the light, the interconversion can be fully reversed. Due to the significant differences between the photoswitched conformations, the kinetics of the interconversion between the triangular ring and rhombicuboctahedral sphere exhibit substantial dissimilarities.

5 The work presented in this chapter has been submitted: M. Han, Y. Luo, B. Damaschke, L. Gómez, X. Ribas, A. Jose, P. Peretzki, M. Seibt, G. H. Clever, Angew. Chem. Int. Ed. 2016, 55, 445. (VIP, Inside Cover) 


\subsection{Introduction}

As discussed in previous chapters, particular external stimuli such as light,[1] electrical fields, ${ }^{[2]}$ mechanical forces,[3] sound, ${ }^{[4]}$ temperature, ${ }^{[5]}$ electrical input (redox) ${ }^{[6]}$ or the addition of small molecules[7] (including acids and bases ${ }^{[8]}$ ) can be used to control the architectures of self-assemblies. Among these numerous external stimuli, light is of special interest undoubtedly because it can work cleanly, rapidly, remotely, and noninvasively. A plethora of self-assembled ring or cages has been reported, which is responsive to light. A light signal has been used to trigger structural changes of self-assembled rings ${ }^{[9]}$, to control over the gelation of nanocages,[10] to alter the hydrophobicity of the complex's cavity, ${ }^{[11]}$ and to modulate guest binding. ${ }^{[12-14]}$

In Chapter 4, a dithienylethene (DTE) ${ }^{[15][16]}$ photoswitch was introduced into pyridinebased ligand $\mathbf{L}^{\mathbf{1}}$, which upon addition of $\mathrm{Pd}^{\mathrm{II}}$ cation with stoichiometric amounts, selfassembles into $\operatorname{Pd}_{2} \mathbf{L}_{4}{ }_{4}$ cage.[17] When $\operatorname{Pd}_{2}\left(o-\mathbf{L}^{1}\right)_{4}$ was irradiated at $365 \mathrm{~nm}$ UV light, ring cyclisation of the photoswitches occurred, resulting all four ligands to rigidify. Both open$\mathrm{Pd}_{2}\left(o-\mathbf{L}^{\mathbf{1}}\right)_{4}$ and closed-form $\operatorname{Pd}_{2}\left(c-\mathbf{L}^{\mathbf{1}}\right)_{4}$ cage bind guest $\left[\mathrm{B}_{12} \mathrm{~F}_{12}\right]^{2-}$, but with totally different affinities. In this case, however, the metal-ligand ratio remained unchanged upon irradiation.
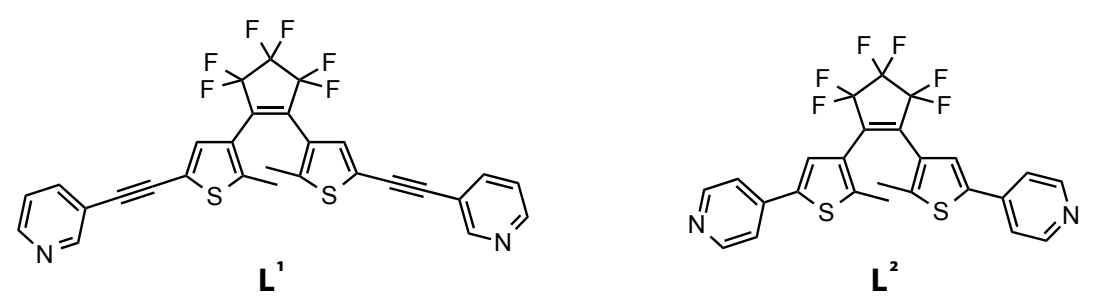

In this chapter, a light-triggered clean structural interconversion[18-20] between selfassemblies with different size, shape and nulearity will be discussed, in which a new derivative ligand $\mathbf{L}^{2}$, containing the same DTE photoswitch as in $\mathbf{L}^{\mathbf{1}}$ but carrying two parasubstituted pyridyl donors, was used. This ligand has been reported several times, which has been used as nondestructive readout photochemical devices,[21][22] as molecular switch with advanced logic functions ${ }^{[23]}$ and in $\mathrm{Pt}^{\mathrm{II}}$ complexes for photoactivated chemotherapy (PACT),[24] but not yet in the preparation of self-assembled structures so far. It is worth mentioning that the non-fluorinated variant of $\mathbf{L}^{2}$ has been used in photochromic metalorganic frameworks, for reversible control of oxygen generation[25] and for dynamic control of the energy transfer.[26] However, most of these applications benefit from the substantial change upon irradiation between a non-conjugated open form and a $\pi$ conjugated closed form. Applications make use of the great change of conformational flexibility/rigidity, has been barely exploited.[15][17][27][28] 


\subsection{Synthesis}

Bis-monodentate pyridyl ligand $o-\mathbf{L}^{2}$ based on a dithienylethene photoswitch was synthesized from perfluoro-1,2-bis(2-iodo-5-methylthien-4-yl)cyclopentene and 4pyridineboronic acid pinacol ester by a Suzuki cross-coupling reaction. The free ligand $o$ $\mathbf{L}^{2}$ could be converted from its open-form into the closed photoisomer $c-\mathbf{L}^{2}$ by irradiation with UV light of $313 \mathrm{~nm}$ wavelength within 5 min using a $500 \mathrm{~W}$ mercury pressure lamp (Figure 6-1). Reaction monitoring by ${ }^{1} \mathrm{H}$ NMR spectroscopy showed that the conversion of the yellowish $o-\mathbf{L}^{2}$ isomer into the intense blue closed-form $c-\mathbf{L}^{2}$ is high yielding $(>96 \%$; Figure 6-2). When the reverse isomerization was triggered by irradiation with light of $617 \mathrm{~nm}$ wavelength, $c$ - $\mathbf{L}^{2}$ was converted back into the open-form isomer $o$ - $\mathbf{L}^{2}$ completely (Figure 6-2).
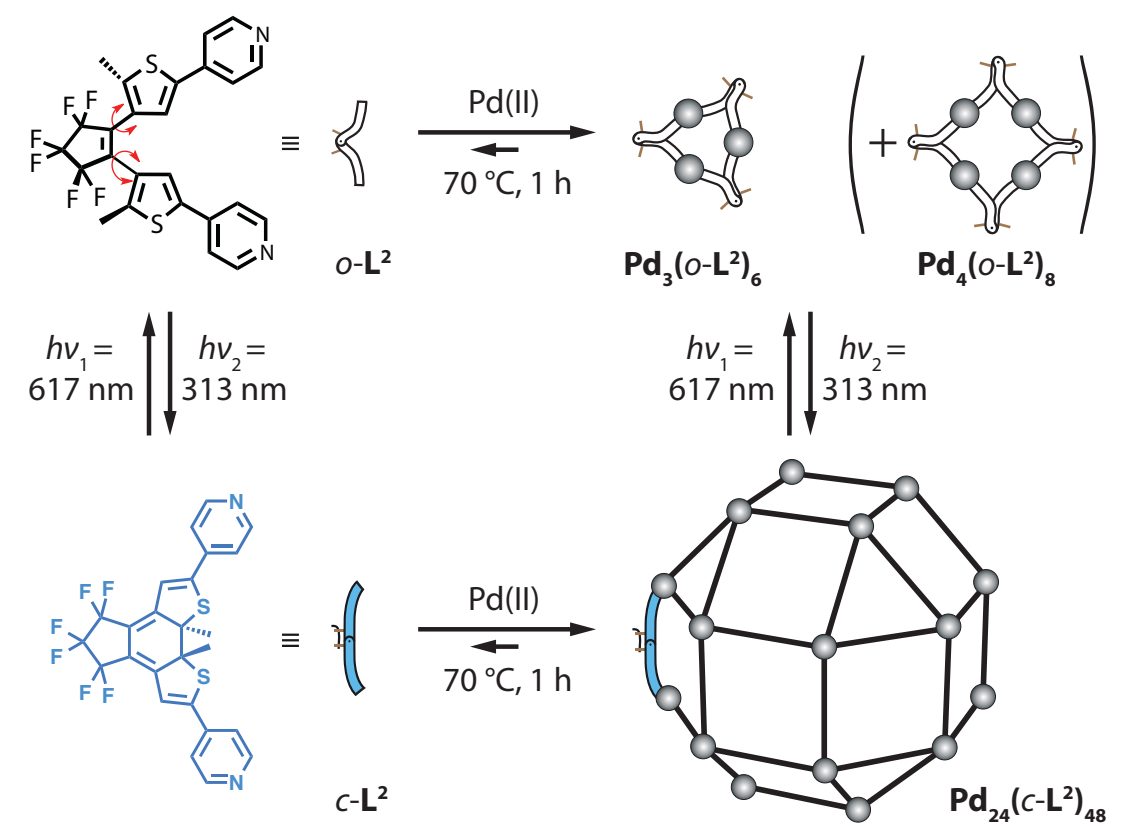

Figure 6-1 Photoisomerization and self-assembly of the ligand photoisomers into the triangular ring $\operatorname{Pd}_{3}\left(o-\mathbf{L}^{2}\right)_{6}$ and the rhombicuboctahedral sphere $\operatorname{Pd}_{24}\left(c-\mathbf{L}^{2}\right)_{48}$ and their structural interconversion upon light irradiation. Copyright (C) 2016 WILEY-VCH Verlag GmbH \& Co.

By treating a mixture of ligand $o-\mathbf{L}^{2}$ with $\left[\mathrm{Pd}\left(\mathrm{CH}_{3} \mathrm{CN}\right)_{4}\right]\left(\mathrm{BF}_{4}\right)_{2}$ in a $2: 1$ ratio in $\mathrm{CD}_{3} \mathrm{CN}$ followed by heating for $1 \mathrm{~h}$ at $70^{\circ} \mathrm{C}$, a mixture of two highly symmetric products was formed as evidenced by NMR (Figure 6-2). In the resulting ${ }^{1} \mathrm{H}$ NMR spectrum, the downfield shift of the pyridine signals was attributed to Pd-ligand complexation.[29] The signal of the thiophene rings was found to be shifted significantly upfield in the two species $(\Delta \delta=-0.25 \mathrm{ppm}$ and $-0.23 \mathrm{ppm}$, respectively). 


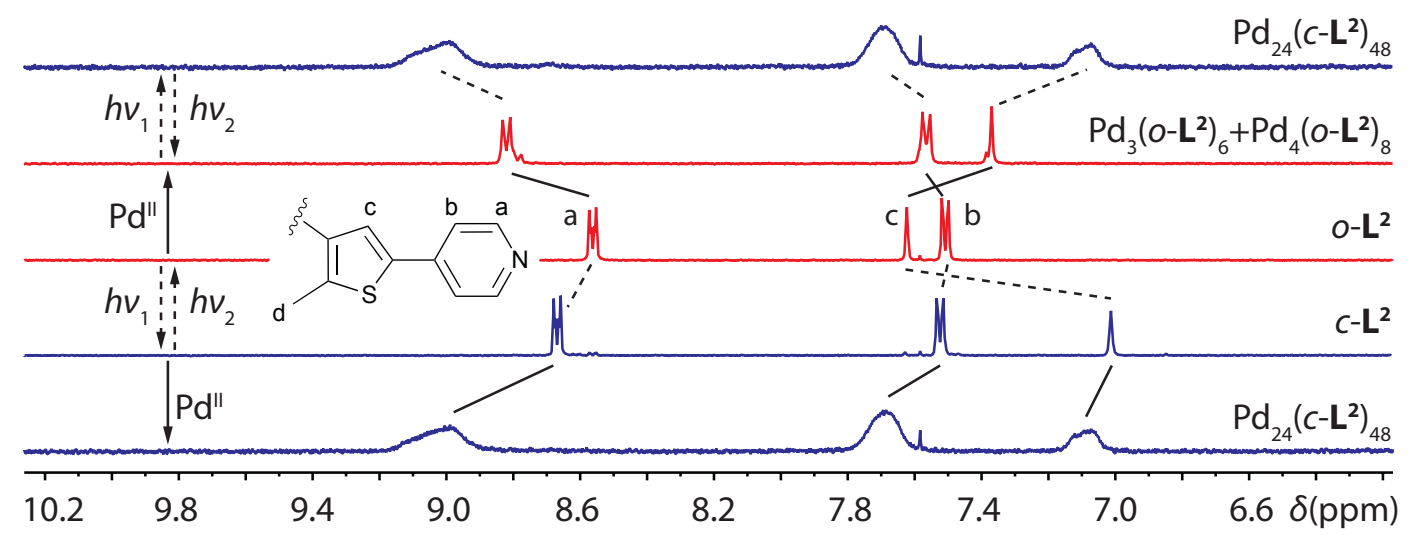

Figure 6-2 ${ }^{1} \mathrm{H}$ NMR spectra $\left(300 \mathrm{MHz}, \mathrm{CD}_{3} \mathrm{CN}, 300 \mathrm{~K}\right)$ of ligands $\boldsymbol{o}-\mathbf{L}^{2}, c-\mathbf{L}^{2}$ and selfassembled cages $\mathrm{Pd}_{3}\left(o-\mathbf{L}^{2}\right)_{6}, \mathrm{Pd}_{24}\left(c-\mathbf{L}^{2}\right)_{48}$. Copyright (C) 2016 WILEY-VCH Verlag GmbH \& Co.

From the ESI mass spectrum, the formation of a mixture of three- and four-membered rings $\left[\mathrm{Pd}_{3}\left(o-\mathbf{L}^{2}\right)_{6}\right]\left(\mathrm{BF}_{4}\right)_{6}$ and $\left[\mathrm{Pd}_{4}\left(o-\mathbf{L}^{2}\right)_{8}\right]\left(\mathrm{BF}_{4}\right)_{8}$ was confirmed through a series of prominent peaks, namely $\left[\mathrm{Pd}_{3}\left(o-\mathbf{L}^{2}\right)_{6}+n\left(\mathrm{BF}_{4}\right)\right]^{(6-n)+}(n=1-4)$ and $\left[\mathrm{Pd}_{4}\left(o-\mathbf{L}^{2}\right)_{8}+n\left(\mathrm{BF}_{4}\right)\right]^{(8-n)+}$ $(n=2-4) .[30-35]$

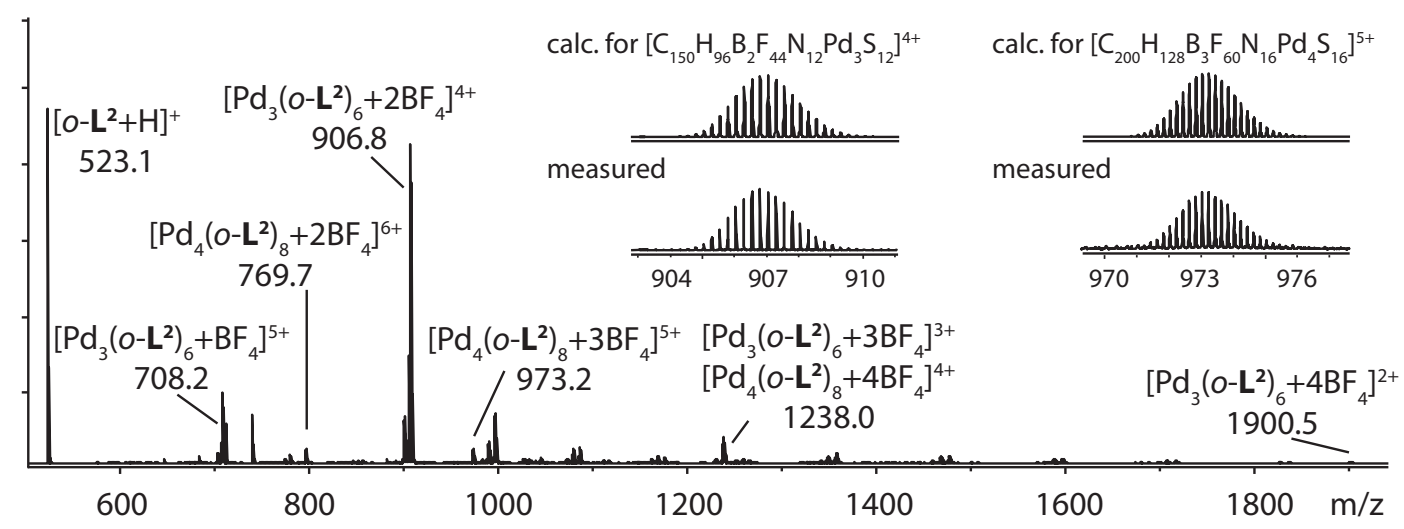

Figure 6-3 ESI mass spectra of open cage mixture $\operatorname{Pd}_{3}\left(o-\mathbf{L}^{2}\right)_{6}$ and $\operatorname{Pd}_{4}\left(o-\mathbf{L}^{2}\right)_{8}$ with $\mathrm{BF}_{4}{ }^{-}$as counteranions. The inset shows the simulated and observed isotopic patterns of $\left[\mathrm{Pd}_{3}\left(\mathrm{o}-\mathbf{L}^{2}\right)_{6}+2 \mathrm{BF}_{4}\right]^{4+}$ and $\left[\mathrm{Pd}_{4}\left(o-\mathbf{L}^{2}\right)_{8}+3 \mathrm{BF}_{4}\right]^{5+}$. Copyright (C) 2016 WILEY-VCH Verlag GmbH $\&$ Co.

The ratio of the two self-assembled products was determined to be $3: 1$ $\left(\mathrm{Pd}_{3}\left(o-\mathbf{L}^{2}\right)_{6}: \mathrm{Pd}_{4}\left(o-\mathbf{L}^{2}\right)_{8}\right)$ by the integral ratio extracted from the $400 \mathrm{MHz}$ and $500 \mathrm{MHz}$ NMR spectra (Figure 6-4). 

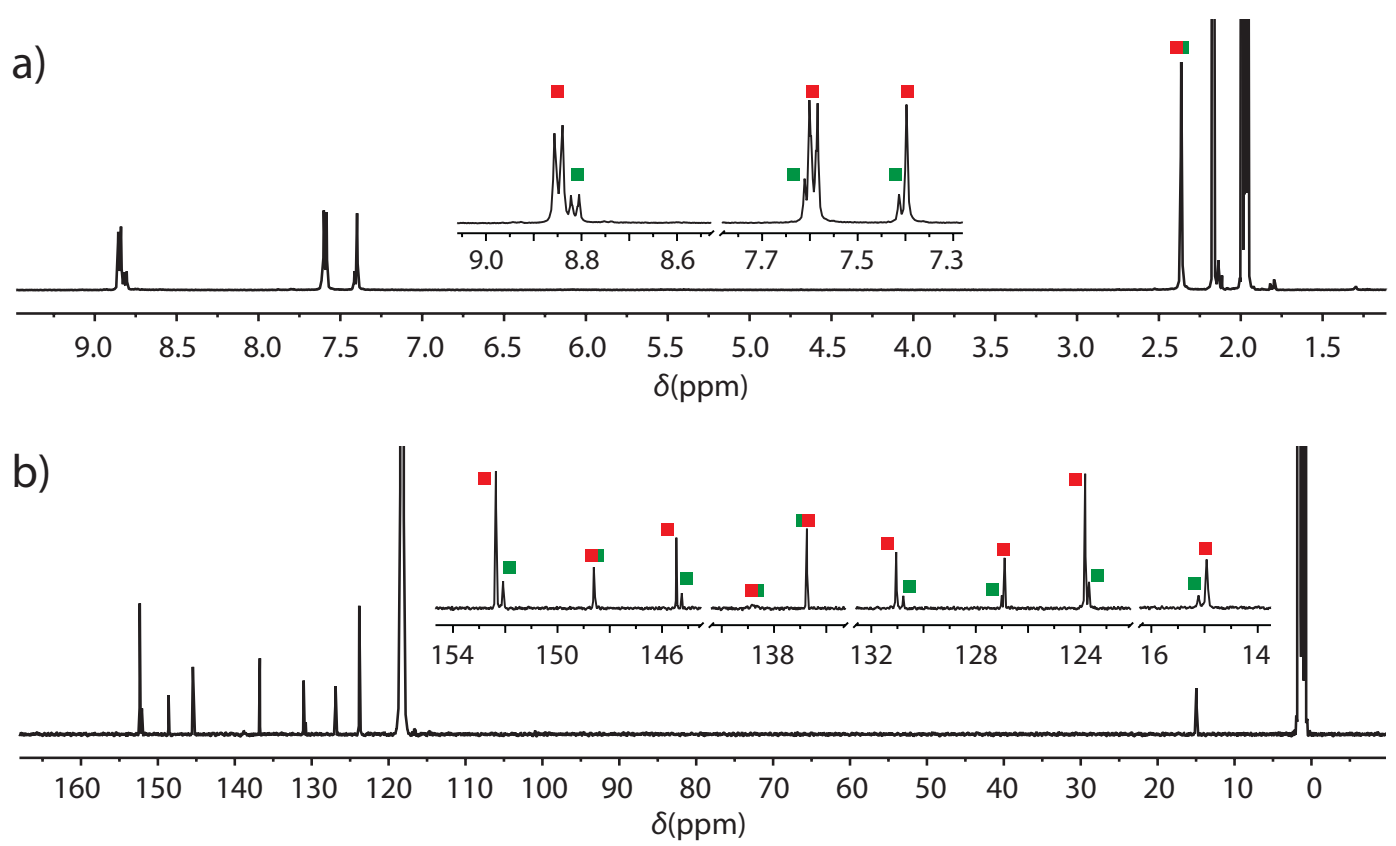

Figure 6-4 a) ${ }^{1} \mathrm{H}$ NMR spectrum $\left(400 \mathrm{MHz}, \mathrm{CD}_{3} \mathrm{CN}, 296 \mathrm{~K}\right)$ and b) ${ }^{13} \mathrm{C}$ NMR spectrum (125 MHz, CD 3 CN, $296 \mathrm{~K})$ of $\operatorname{Pd}_{3}\left(o-\mathbf{L}^{2}\right)_{6}$ (red) and $\operatorname{Pd}_{4}\left(o-\mathbf{L}^{2}\right)_{8}$ (green).

Diffusion-ordered NMR spectroscopy (DOSY) also revealed the formation of the mixture (Figure 6-5): the two ring sizes could be clearly differentiated by their different diffusion coefficient, yielding $4.40 \times 10^{-10} \mathrm{~m}^{2} \mathrm{~s}^{-1}$ for the smaller and $3.86 \times 10^{-10} \mathrm{~m}^{2} \mathrm{~s}^{-1}$ for the larger species, thus indicating the formation of structures with hydrodynamic radii of $1.34 \mathrm{~nm}$ and $1.52 \mathrm{~nm}$, respectively (in $\mathbf{5 . 7 . 4}$ ).

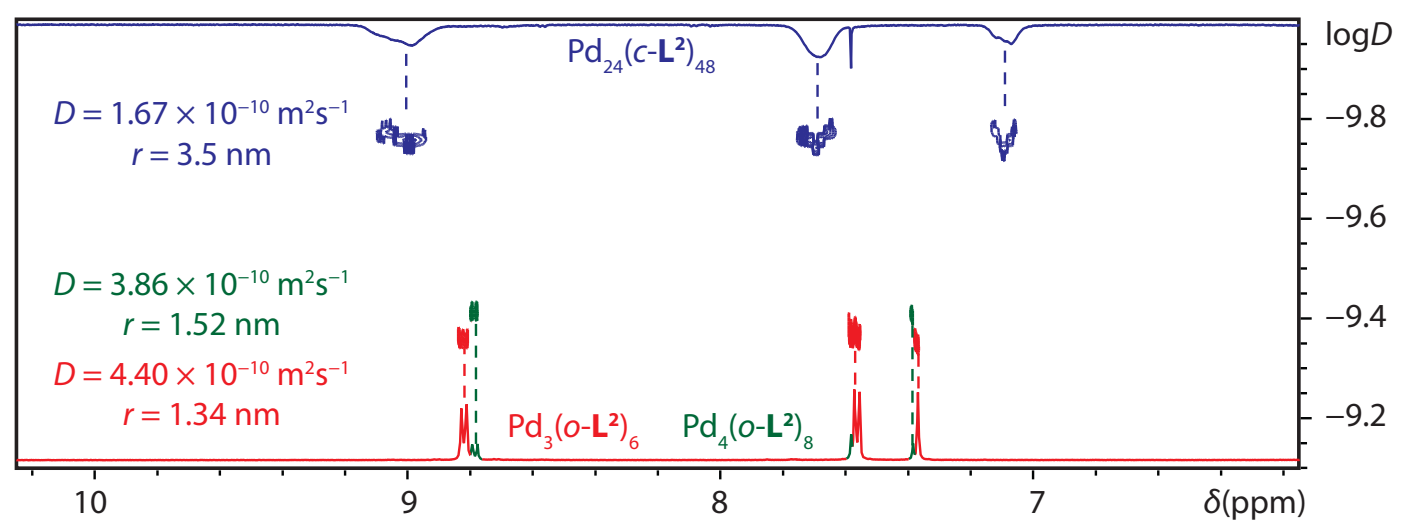

Figure 6-5 Superposition of ${ }^{1} \mathrm{H}$ DOSY NMR spectra of $\operatorname{Pd}_{3}\left(o-\mathbf{L}^{2}\right)_{6}, \operatorname{Pd}_{4}\left(o-\mathbf{L}^{2}\right)_{8}$ and $\mathrm{Pd}_{24}\left(c-\mathbf{L}^{2}\right)_{48}\left(400 \mathrm{MHz}, \mathrm{CD}_{3} \mathrm{CN}, 296 \mathrm{~K}\right)$. Copyright (C) 2016 WILEY-VCH Verlag GmbH \& Co.

When $\mathrm{Pd}\left(\mathrm{NO}_{3}\right)_{2} \cdot \mathrm{H}_{2} \mathrm{O}$ was used as the metal source instead of $\left[\mathrm{Pd}\left(\mathrm{CH}_{3} \mathrm{CN}\right)_{4}\right]\left(\mathrm{BF}_{4}\right)_{2}$ for the self-assembly in $\mathrm{CD}_{3} \mathrm{CN}$, only one product was formed according to the ${ }^{1} \mathrm{H}$ NMR spectrum (Figure 6-6). ESI mass spectrometry indicates the exclusive formation of the 3-ring 
$\left[\mathrm{Pd}_{3}\left(o-\mathbf{L}^{2}\right)_{6}\right]\left(\mathrm{NO}_{3}\right)_{6}$ by showing isotopically well resolved peaks for the species $\left[\mathrm{Pd}_{3}\left(o-\mathbf{L}^{2}\right)_{6}+n\left(\mathrm{NO}_{3}\right)\right]^{(6-n)+}(n=2-4)$ (Figure 6-7). Anion-templating effects are suspected to be responsible for the control over the sizes of the formed rings.[36] Unfortunately, the intended studies on the photochemistry of the nitrate-based cage were hampered by the formation of precipitates accompanied by vanishing of all ${ }^{1} \mathrm{H}$ NMR signals except the solvent peak upon irradiation (Figure 6-6). Therefore, all further experiments were carried out using the mixture of $\mathrm{Pd}_{3}\left(o-\mathbf{L}^{2}\right)_{6}: \mathrm{Pd}_{4}\left(o-\mathbf{L}^{2}\right)_{8}$ with $\mathrm{BF}_{4}^{-}$as the counter anion.

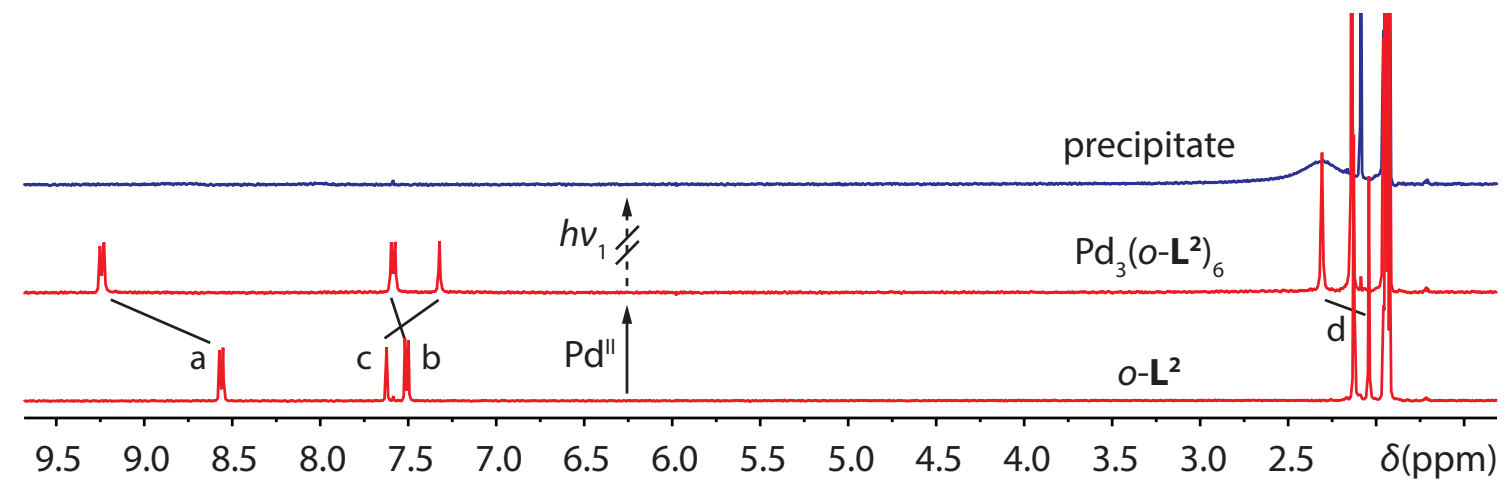

Figure 6-6 ${ }^{1} \mathrm{H}$ NMR spectra (300 MHz, $\left.\mathrm{CD}_{3} \mathrm{CN}, 300 \mathrm{~K}\right)$ ligand $o$ - $\mathbf{L}^{2}$, self-assembled cage $\left[\mathrm{Pd}_{3}\left(o-\mathbf{L}^{2}\right)_{6}\right]\left(\mathrm{NO}_{3}\right)_{6}$ and its spectrum after irradiation with $313 \mathrm{~nm}$ light.

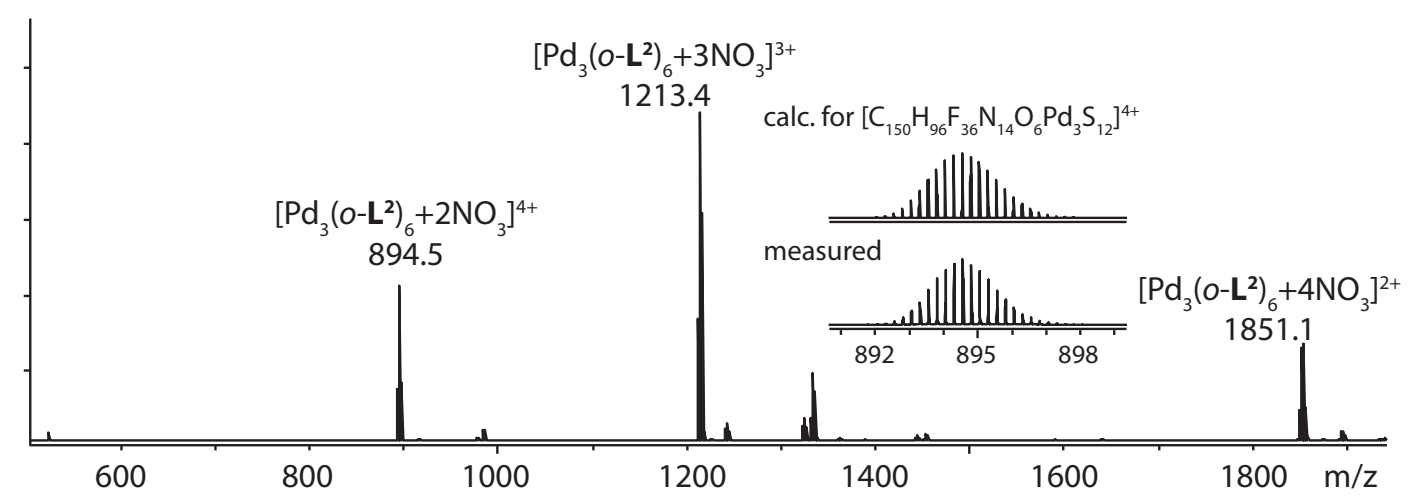

Figure 6-7 ESI mass spectrum of open cage $\mathrm{Pd}_{3}\left(o-\mathbf{L}^{2}\right)_{6}$ with $\mathrm{NO}_{3}{ }^{-}$as counter anion. The inset shows the simulated and observed isotopic pattern of $\left[\mathrm{Pd}_{3}\left(o-\mathbf{L}^{2}\right)_{6}+2 \mathrm{NO}_{3}\right]^{4+}$.

Later on, the large self-assembly constructed from the closed-form ligand $c-\mathbf{L}^{2}$ and $\mathrm{Pd}^{\mathrm{II}}$ has been studied. A mixture of $c-\mathbf{L}^{2}$ and $\left[\mathrm{Pd}\left(\mathrm{CH}_{3} \mathrm{CN}\right)_{4}\right]\left(\mathrm{BF}_{4}\right)_{2}$ in a $2: 1$ ratio was heated in $\mathrm{CD}_{3} \mathrm{CN}$, which resulted a downfield shift of all aromatic proton signals, occurred with very strong signal broadening (Figure 6-2). The ${ }^{1} \mathrm{H}$ DOSY NMR spectrum clearly showed signals assignable to a single product with a diffusion constant of $D=1.67 \times 10^{-10} \mathrm{~m}^{2} \mathrm{~s}^{-1}$, which indicates the formation of a larger supramolecule with a hydrodynamic radius of $3.5 \mathrm{~nm}$ in $\mathrm{CD}_{3} \mathrm{CN}$ (Figure 6-5). Therefore, the formation of a large rhombicuboctahedral 
sphere of the composition $\operatorname{Pd}_{24}\left(c-\mathbf{L}^{2}\right)_{48}{ }^{[37]}$ could be considered. This hypothesis is in good accordance with the self-assembled spherical structures based on similar bismonodentate ligands introduced by Fujita and coworkers.[38] According to their empirical prediction and results, they found out the most important parameter that determines the final self-assembled structure is the bend angle $\theta$ of the ligand component. In their previous work, ${ }^{[39]}$ they have shown that the bend angle values below $127^{\circ}$ result $M_{12} \mathbf{L}_{24}$ structures, whereas those above $135^{\circ}$ give $M_{24} \mathbf{L}_{48}$ structures (Figure 6-8). Recently, they narrowed the range of bend angle of the sharp structural switch between cuboctahedron and rhombicuboctahedron within just $4^{\circ}$. It was shown that ligand angles smaller than about $130^{\circ}$ lead to the formation of $\operatorname{Pd}_{12} \mathbf{L}_{24}$ whereas the ligands with angles $>134^{\circ}$ results in the formation of larger $\operatorname{Pd}_{24} \mathbf{L}_{48}$ supramolecular spheres.[40]

a)

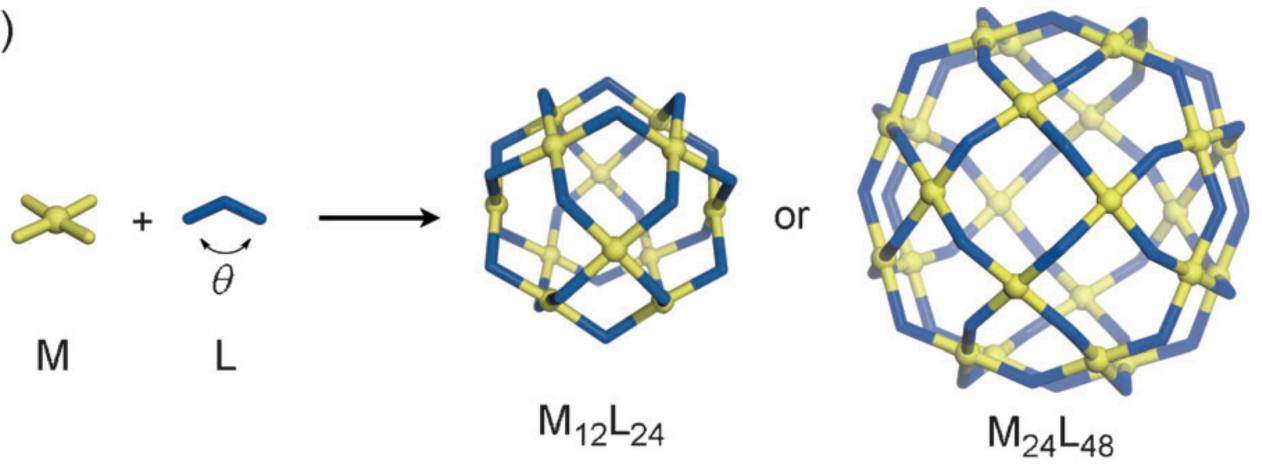

b)

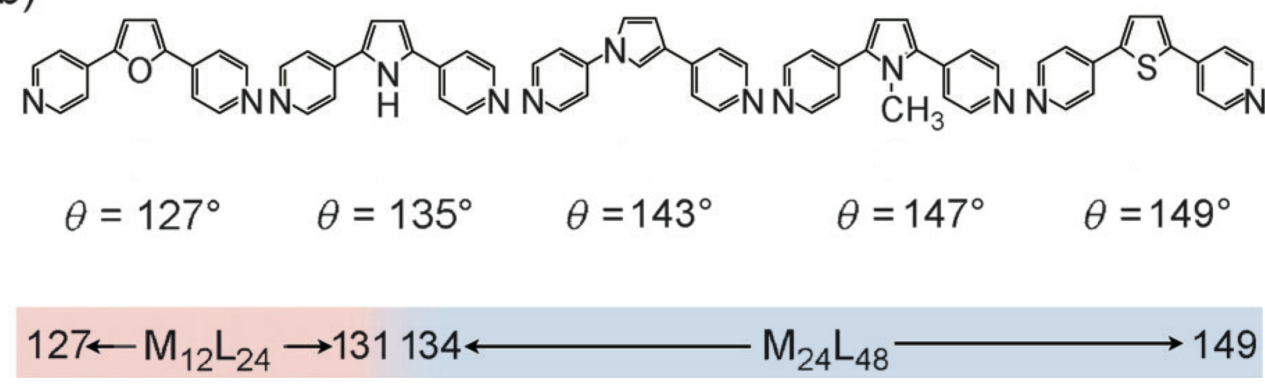

Figure 6-8 a) Representation of self-assembly of $M_{12} \mathbf{L}_{24}$ and $M_{24} \mathbf{L}_{48}$ polyhedra. b) Structural formation of ligands with different bend angles. Copyright (C) 2012 WILEY-VCH Verlag GmbH \& Co. KGaA, Weinheim.[39]

The DFT calculation (EDF2/6-31G*) revealed that the bend angle in the closed ligand $c$-L has the value of $138.2^{\circ}$, which speaks here clearly for the formation of a large rhombicuboctahedral sphere $\operatorname{Pd}_{24}\left(c-\mathbf{L}^{2}\right)_{48}$. Subsequently, a geometry optimized structure of a $\mathrm{Pd}_{24}\left(c-\mathbf{L}^{2}\right)_{48}$ cage on the semiemperical PM6 level of theory was found to give a radius of $3.2 \mathrm{~nm}$, thus matching very well the $3.5 \mathrm{~nm}$ radius obtained from the DOSY NMR experiment (Figure 6-9b). 

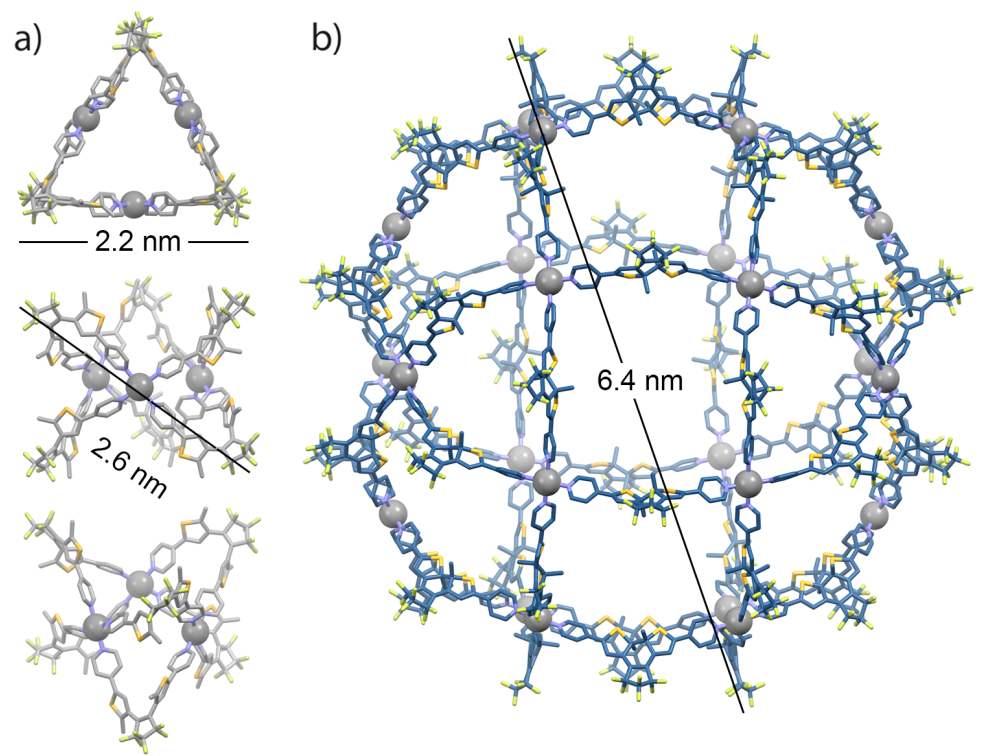

Figure 6-9 a) Different views of the DFT structure calculated for the three-ring compound $\operatorname{Pd}_{3}\left(o-\mathbf{L}^{2}\right)_{6}$. b) Semiemperical (PM6) geometry-optimized molecular model of $\operatorname{Pd}_{24}\left(c-\mathbf{L}^{2}\right)_{48}$. Copyright (C) 2016 WILEY-VCH Verlag GmbH \& Co.

Difficulties were faced during the measurement of mass spectrometry. It was tried many times under different mild conditions in order to achieve an ESI mass spectrum with appropriate resolved isotopic patterns. When an acetonitrile sample of the large closedform self-assembly with a $0.08 \mathrm{mM}$ concentration was subjected ESI mass spectrometry with a standard ESI source (Bruker maXis), two series of conjugated peaks were interestingly observed. One series of them was found with peaks displaying the expected isotopic pattern at $m / z=1238.38,1358.68,1503.45,1679.73,1901.07$, and 2184.79 belonging respectively to $\left[\operatorname{Pd}_{12}\left(c-\mathbf{L}^{2}\right)_{24}+n\left(\mathrm{BF}_{4}\right)\right]^{(24-n)+}(n=12-17)$ corresponding to the formulation of the smaller species (red dots in Figure 6-10), and another series of peaks at $m / z=1332.9,1442.2,1569.4,1720.0,1900.6,2121.7$ was assigned to $\left[\operatorname{Pd}_{15}\left(c-\mathbf{L}^{2}\right)_{30}+n\left(\mathrm{BF}_{4}\right)\right]^{(30-n)+}(n=16-21)$ (blue stars in Figure 6-10). The applied mass spectrometric method obviously caused fragmentation of the very heavy major species $\operatorname{Pd}_{24}\left(c-\mathbf{L}^{2}\right)_{48}$, which has a molecular weight of $31.802 \mathrm{D}$ including all $\mathrm{BF}_{4^{-}}$counter anions. Only one signal at $m / z=2033.34$ was actually in accordance with the calculated mass for the target species $\left.\left[\mathrm{Pd}_{24}\left(c-\mathbf{L}^{2}\right)_{48}+33 \mathrm{BF}_{4}\right)\right]^{15+}$ but the sole occurrence of this peak showing a badly resolved isotopic pattern did not allow us to unambiguously confirm this assignment. 


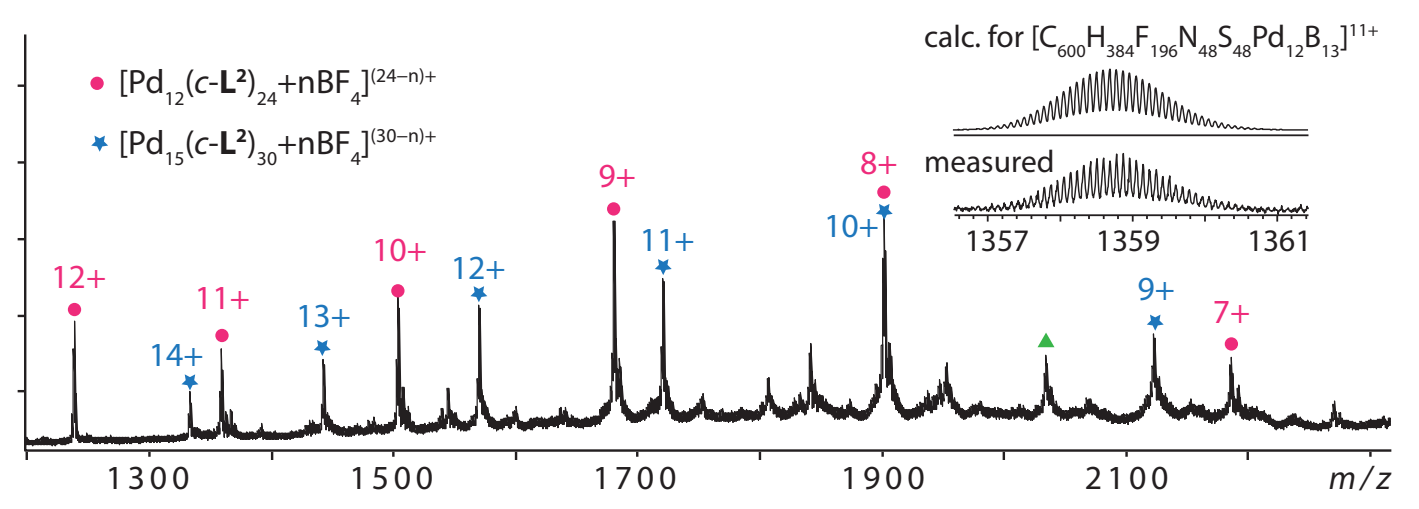

Figure 6-10 ESI mass spectra with standard ESI source at elevated temperature (dry gas temperature $150{ }^{\circ} \mathrm{C}$ ) shows the fragment peaks of $\operatorname{Pd}_{12}\left(c-\mathbf{L}^{2}\right)_{24}$ (pink dots) and $\operatorname{Pd}_{15}\left(c-\mathbf{L}^{2}\right)_{30}$ (blue stars). (green triangle: tentative $\left.\operatorname{Pd}_{24}\left(c-\mathbf{L}^{2}\right)_{48}\right)$. The inset shows the simulated and observed isotopic pattern of $\left[\operatorname{Pd}_{12}\left(c-\mathbf{L}^{2}\right)_{24}+13 \mathrm{BF}_{4}\right]^{11+}$.

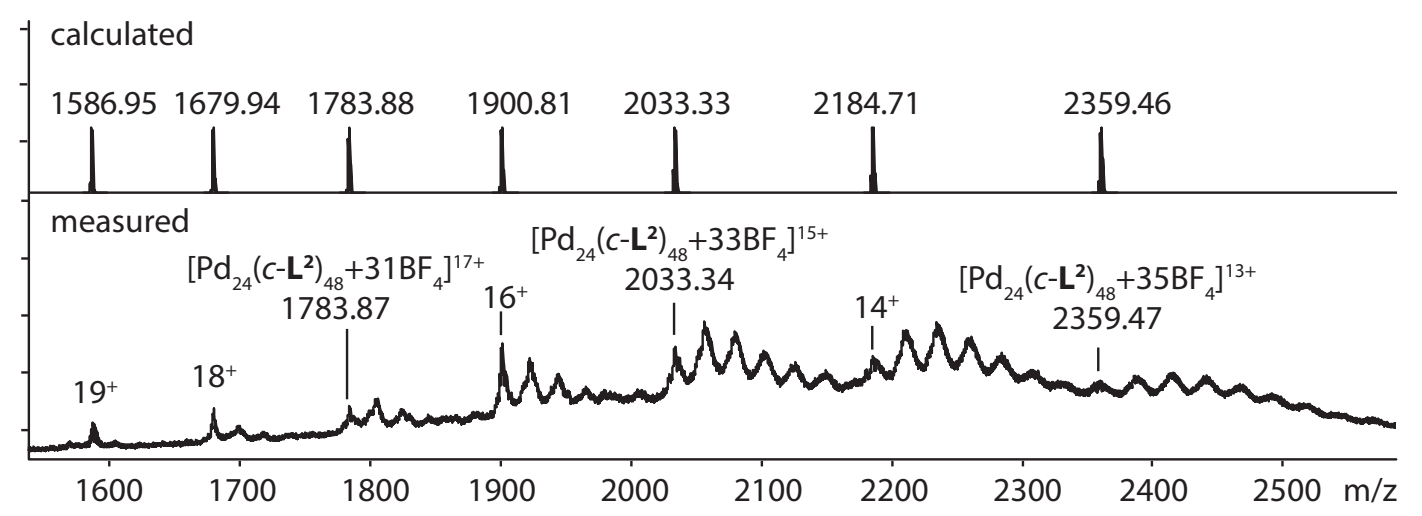

Figure 6-11 Cryospray ionization mass spectrum of large sphere $\operatorname{Pd}_{24}\left(c-\mathbf{L}^{2}\right)_{48}$ with $\mathrm{BF}_{4}{ }^{-}$as counter anions. The positions of the measured peaks with charges between +19 and +13 are assigned and marked with the simulated peak positions. Copyright (C) 2016 WILEYVCH Verlag GmbH \& Co.

In order to get a better result, same acetonitrile sample of $\operatorname{Pd}_{24}\left(c-\mathbf{L}^{2}\right)_{48}$ was measured by a cryospray ionization mass spectroscopy (CSI-MS). ${ }^{6}$ A Bruker cryospray attachment was used for CSI-MS. The temperature of the nebulizing and drying gases was set at $-40{ }^{\circ} \mathrm{C}$. A clean spectrum was obtained with peaks at $m / z=1586.95,1679.98,1783.87,1900.81$, 2033.34, 2184.74, and 2359.47 were assigned to $\left[\operatorname{Pd}_{24}\left(c-\mathbf{L}^{2}\right)_{48}+n\left(\mathrm{BF}_{4}\right)\right]^{(48-n)+}(n=29-35)$,

\footnotetext{
${ }^{6}$ The CSI-MS measurement was carried out by Laura Gómez, Serveis Tècnics de Recerca (STR), Universitat de Girona.
} 
respectively (Figure 6-11). Most of these peaks appear as the first in a series of signals that points to the formation of adducts with a species summing up to a molecular weight of about 345 .

\subsection{Symmetry considerations}

Due to symmetry considerations between a cuboctahedron and a rhombicuboctahedron, the NMR signals of species $\operatorname{Pd}_{12}\left(c-\mathbf{L}^{2}\right)_{24}$ should be distinguishable from $\operatorname{Pd}_{24}\left(c-\mathbf{L}^{\mathbf{2}}\right)_{48}{ }^{[38,39]}$ In the smaller cuboctahedral complex, only one set of signal is expected for each symmetryequivalent ligand proton, since all edges are equal (Figure 6-12c).

However, beyond the intrinsic $3 \mathrm{~J}$ and ${ }^{4} \mathrm{~J}$ couplings of the pyridyl rings, the broadened peaks must be resulted by other reasons, as these couplings are very small $\left(3 \mathrm{~J}=6 \mathrm{~Hz}\right.$ and ${ }^{4} \mathrm{~J}=$ $1.5 \mathrm{~Hz}$ ). In the spherical rhombicuboctahedral $\operatorname{Pd}_{24}\left(c-\mathbf{L}^{2}\right)_{48}$, half of the ligands locate at the edges that connect one triangular and one rectangular face of the polyhedron whereas the other half sit at the edges that both sides are rectangular faces (Figure 6-12a). These two different chemical environments of the ligands result in a first level of signal splitting into two sets with equal integral (A and B in Figure 6-12a).
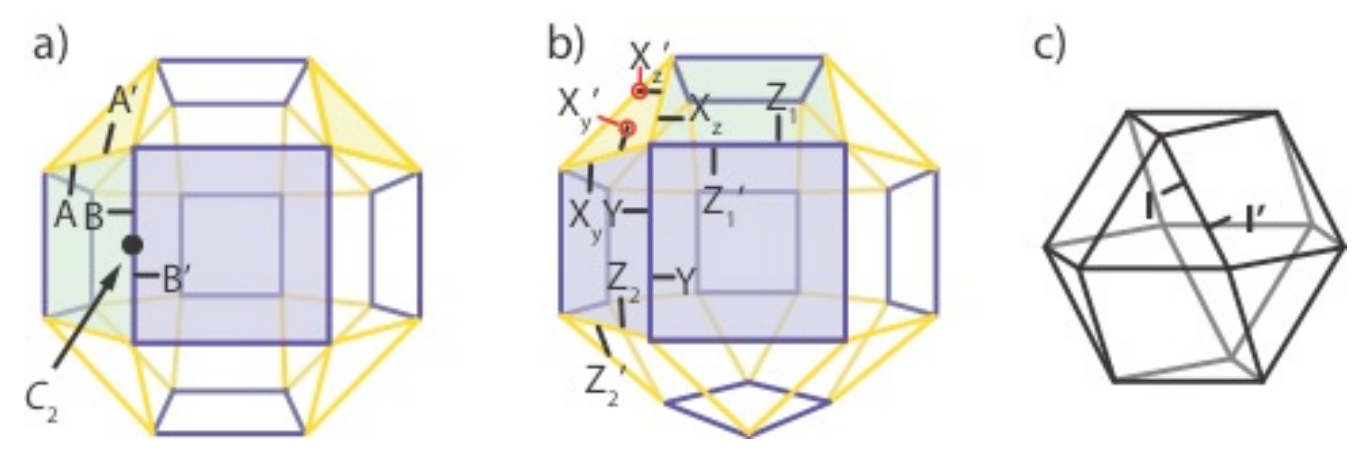

Figure 6-12 Two possible isomers of the homochiral self-assembly $\operatorname{Pd}_{24}\left(c-\mathbf{L}^{2}\right)_{48}$ a) rhombicuboctahedron and b) pseudo-rhombicuboctahedron. The different chemical environments of the ligands halves in both structures are indicated by different colours (yellow: triangles; purple: squares) and further differentiated by the position of the ligands' methyl groups with respect to their neighbourhoods. c) Structure of the hypothetical cuboctahedron $\operatorname{Pd}_{12}\left(c-\mathbf{L}^{2}\right)_{24}$ in which all homochiral ligands occupy equal positions (and only one level of desymmetrization would be expected for the two halves of the $C_{2}$-symmetric ligands).

Furthermore, a second level of splitting arise from the chirality of the $C_{2}$-symmetric ligands, since the disagreement between the ligand's symmetry and the overall cage symmetry, breaks off the chemical equality of the two ligand halves, resulting in 
differentiable A, A', B and B' (Figure 6-12a). More precisely, two adjacent rectangular faces sharing one edge (indicated in green and purple in Figure 6-12a), in which the green rectangle is surrounded by two triangular and two rectangular surfaces, whereas the purple is surrounded by four rectangles. The ligand placed between these green and purple rectangles points its one methyl group into the green, and the other methyl group into the purple rectangle. Thus, this gives a signal splitting into B and B'. In addition, an isomeric form of the large $\operatorname{Pd}_{24}\left(c-\mathbf{L}^{2}\right)_{48}$, sphere, pseudo-rhombicuboctahedron, exists in which one of the square cupola is twisted by $45^{\circ}$ (Figure 6-12b). This twisting further complicates the NMR spectra of the large self-assembly. Same as the rhombicuboctahedral isomer, first level of signal splitting is expected ( $\mathrm{X}$ and $\mathrm{Z}$ in Figure 6-12b), since half of the ligands connect one triangular and one rectangular face of the polyhedron, and the other half connects two rectangular faces. A second level of signal splitting was caused by the chemical inequality of the ligand halves, giving $X_{y}, X_{y}{ }^{\prime}, X_{z}, X_{z}{ }^{\prime}, Z_{1}, Z_{1}^{\prime}, Z_{2}$ and $Z_{2}{ }^{\prime}$ (Figure 6-12b). A third level of signal splitting $Y$ is caused by the ligand sit in between the two adjacent rectangular faces (purple in Figure 6-12b), each of which is surrounded by one triangular face and three rectangular faces. The pseudo-rhombicuboctahedral isomer could give more complicated splitting pattern.

The signal splitting effects could not be clearly investigated, not only because of the coexistence of the isomers with unknown relative ratio and complex splitting patters, but also because of the slowdown of tumbling motion of the large $\operatorname{Pd}_{24}\left(c-\mathbf{L}^{2}\right)_{48}$ complex on the NMR time scale. However, there is another reason for the signal broadening that is not negligible. The rigid photoisomer $c$ - $\mathbf{L}^{2}$ is a configurationally stable, $C_{2}$-symmetric chiral compound. Thus, when the racemic mixture of $c-\mathbf{L}^{2}$ was used directly to synthesize the palladium-mediated self-assembly, formation of hundreds of diastereomers with respect to the $R: S$ ratio of the ligands and their relative position in the assembly could not be difficult imagined.

In order to overcome this problem, the racemic mixture of the photoswitched $c$ - $\mathbf{L}^{2}$ ligands was resolved using chiral HPLC under strict exclusion of daylight (Figure 6-13). After the separation of racemic mixture of $c$ - $\mathbf{L}^{2}$, the cage formation was followed by NMR spectroscopy (Figure 6-14). The signal broadening was reduces to some extent.

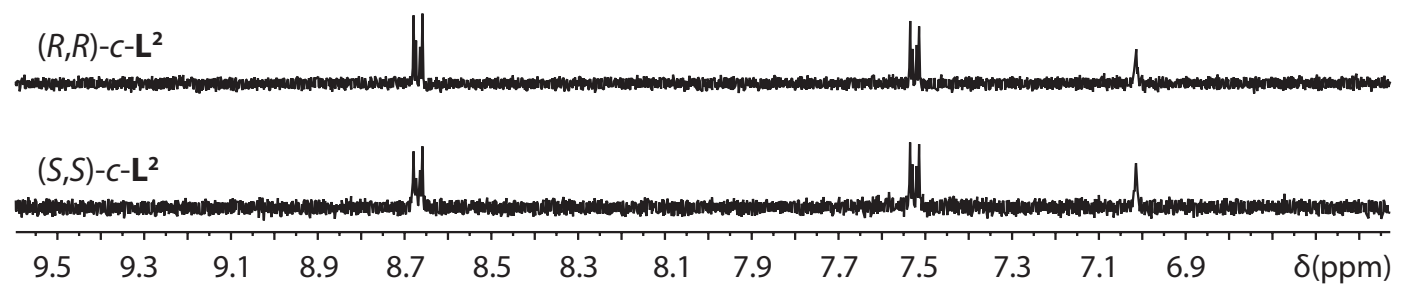

Figure 6-13 ${ }^{1} \mathrm{H}$ NMR spectra $\left(300 \mathrm{MHz}, \mathrm{CD}_{3} \mathrm{CN}\right)$ of $(R, R)$ - and $(S, S)-c-\mathbf{L}^{2}$. 


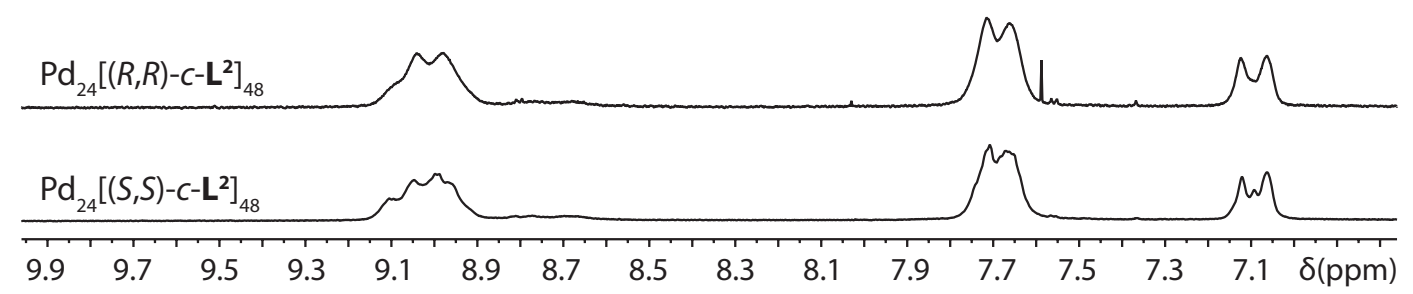

Figure 6-14 ${ }^{1} \mathrm{H}$ NMR spectra $\left(500 \mathrm{MHz}, \mathrm{CD}_{3} \mathrm{CN}\right)$ of $\mathrm{Pd}_{24}\left(c-\mathbf{L}^{2}\right)_{48}$ originated from $(R, R)$ - and $(S, S)-c-\mathbf{L}^{2}$.

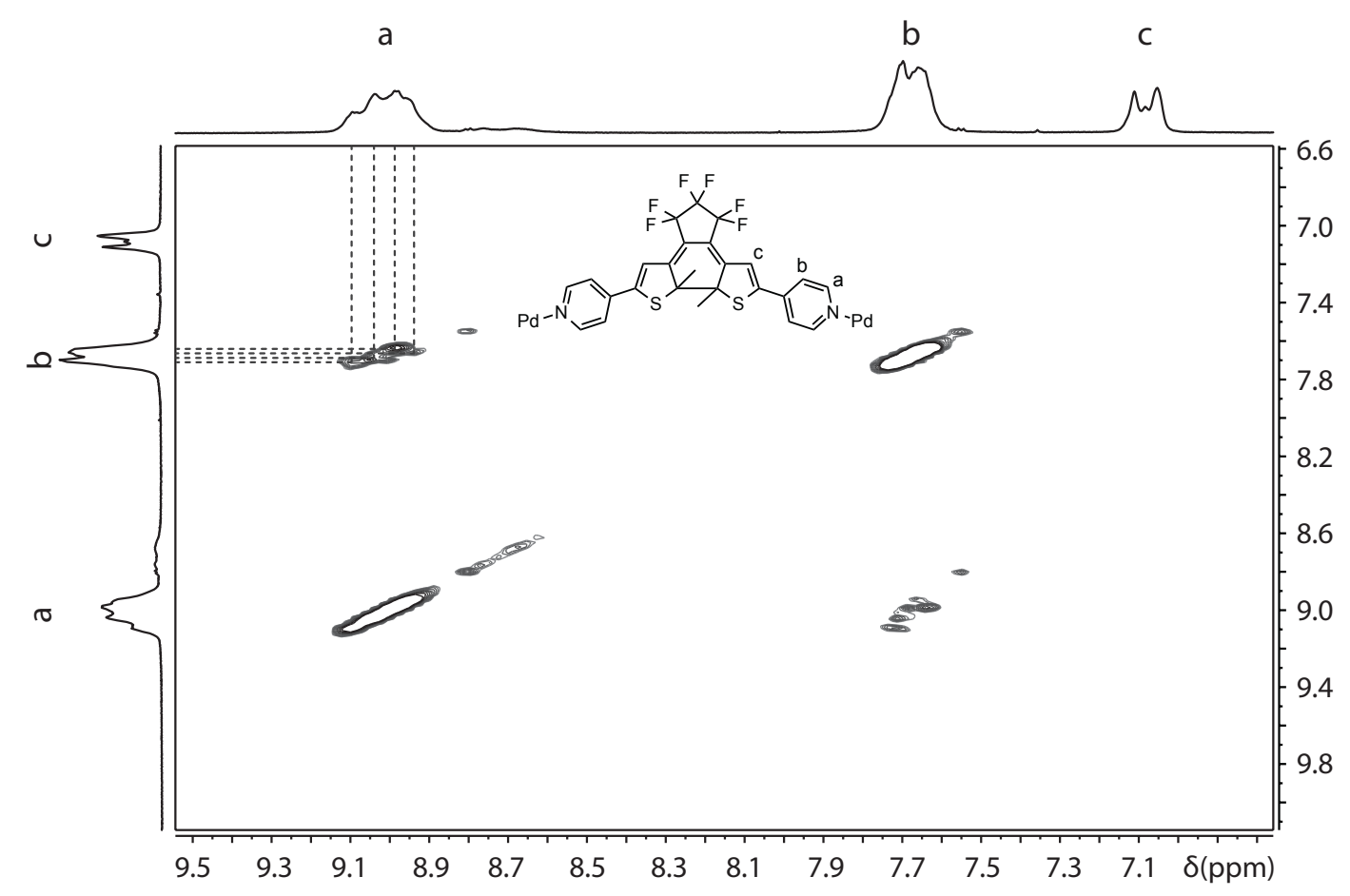

Figure 6-15 The ${ }^{1} \mathrm{H}-{ }^{1} \mathrm{H}$ COSY spectrum $\left(500 \mathrm{MHz}, \mathrm{CD}_{3} \mathrm{CN}\right)$ of $\mathrm{Pd}_{24}\left[(S, S)-c-\mathbf{L}^{2}\right]_{48}$.

2D NMR spectra of the homochiral $\operatorname{Pd}_{24}\left(c-\mathbf{L}^{2}\right)_{48}$ containing one of the isolated enantiomer was measured on a $500 \mathrm{MHz}$ and $900 \mathrm{MHz}$ spectrometers. In the COSY spectrum (Figure 6-15) of $\operatorname{Pd}_{24}\left[(S, S)-c-L^{2}\right]_{48}$, correlations were found between $H_{a}$ and $H_{b}$. The cross-peak split into at least four sets of signals. The similar signal splitting was observed in the 900 $\mathrm{MHz}{ }^{1} \mathrm{H},{ }^{13} \mathrm{C}$ HSQC spectrum (Figure 6-16) of $\mathrm{Pd}_{24}\left[(R, R)-C-\mathbf{L}^{2}\right]_{48}$. Again each proton signal is split up into more than four signals (The chemical shift assignments of the ${ }^{13} \mathrm{C}$ NMR spectrum are written next to the signals). 


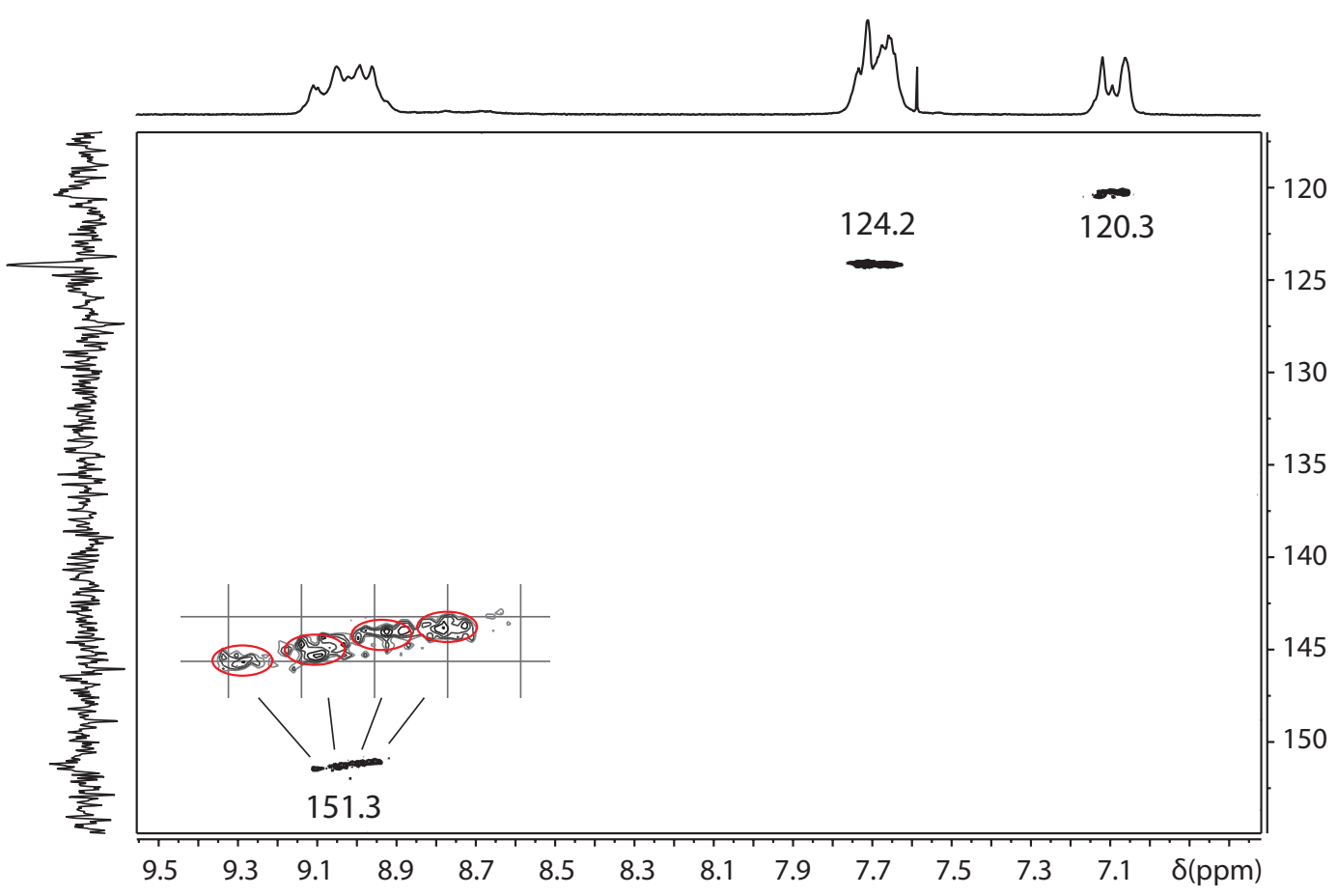

Figure 6-16 The ${ }^{1} \mathrm{H},{ }^{13} \mathrm{C}$ HSQC spectrum $\left(900 \mathrm{MHz}, \mathrm{CD}_{3} \mathrm{CN}\right)$ of $\mathrm{Pd}_{24}\left[(R, R)-c-\mathbf{L}^{2}\right]_{48}$.

And besides, the ${ }^{19} \mathrm{~F}-19 \mathrm{~F}$ COSY spectrum (Figure 6-17) shows correlations between the two flanking $\mathrm{CF}_{2}$ groups and the central $\mathrm{CF}_{2}$ clearly. The correlations between both flanking $\mathrm{CF}_{2}$ groups were also observed.

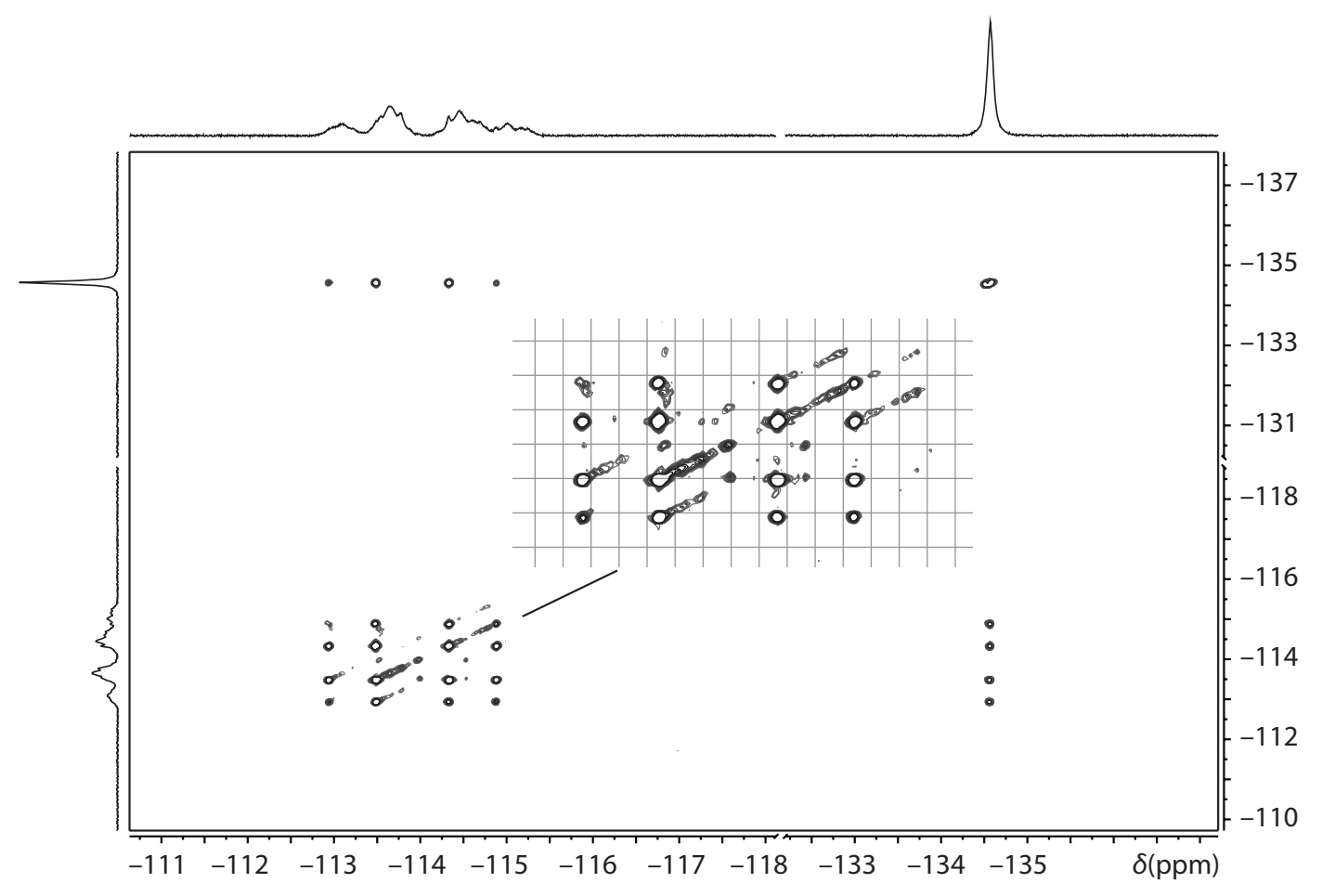

Figure 6-17 The ${ }^{19} \mathrm{~F}-19 \mathrm{~F}$ COSY spectrum $\left(470 \mathrm{MHz}, \mathrm{CD}_{3} \mathrm{CN}\right)$ of $\mathrm{Pd}_{24}\left[(R, R)-c-\mathbf{L}^{2}\right]_{48}$. 


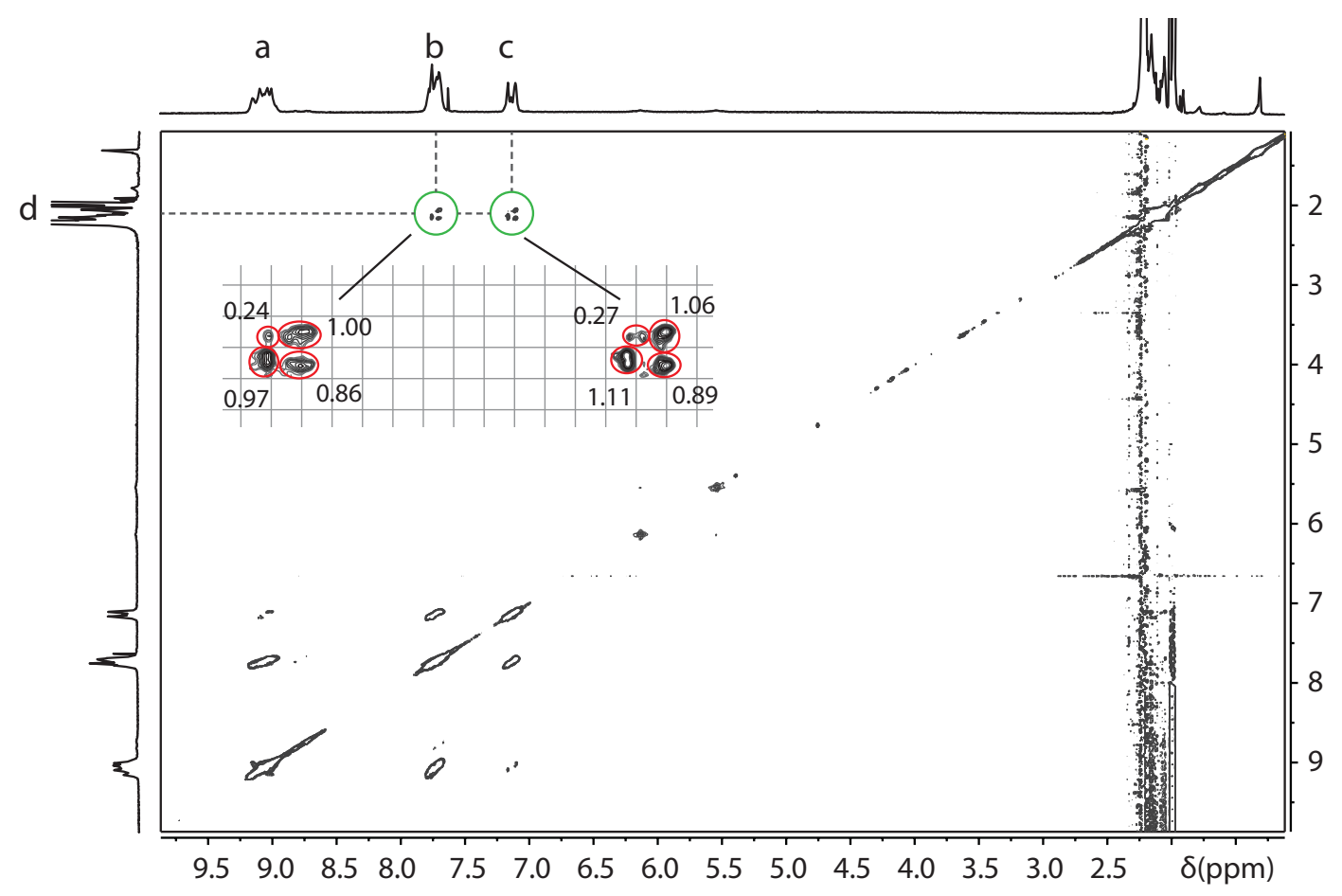

Figure 6-18 The ${ }^{1} \mathrm{H}-{ }^{-1} \mathrm{H}$ NOESY spectrum (900 MHz, $\mathrm{CD}_{3} \mathrm{CN}$ ) of $\mathrm{Pd}_{24}\left[(R, R)-c-\mathbf{L}^{2}\right]_{48}$. Contacts between one of the pyridyl proton $\mathrm{H}_{b}$ and thiophene proton $\mathrm{H}_{c}$ with the methyl group $\mathrm{H}_{\mathrm{d}}$ are highlighted in red.

An NOESY experiment performed on the homochiral cage revealed contacts between one of the pyridyl proton $\mathrm{H}_{\mathrm{b}}$ and thiophene proton $\mathrm{H}_{\mathrm{c}}$ with the methyl group $\mathrm{H}_{\mathrm{d}}$. Each crosspeak was split up to at least four sets of signals with approximate integration (Figure 6-18).

All 2D NMR spectra support the postulation of coexistence of aforementioned rhombicuboctahedron and pseudo-rhombicuboctahedron isomers. However, the relative ratio of such complicated isomers could not be elucidated based on the available measurement data.

\subsection{Other characterization of spherical $\operatorname{Pd}_{24}\left(c-\mathbf{L}^{2}\right)_{48}$}

Numerous attempts to grow crystals of the $\operatorname{Pd}_{24}\left(c-\mathbf{L}^{2}\right)_{48}$ sphere of sufficient quality for single crystal analysis were unsuccessful. Due to the intensively blue coloured solution and the sensitivity of the photochromic $\operatorname{Pd}_{24}\left(C-\mathbf{L}^{2}\right)_{48}$ compound to daylight, obtaining crystals was impeded. 


\subsubsection{Transmission electron microscopy (TEM) ${ }^{7}$}
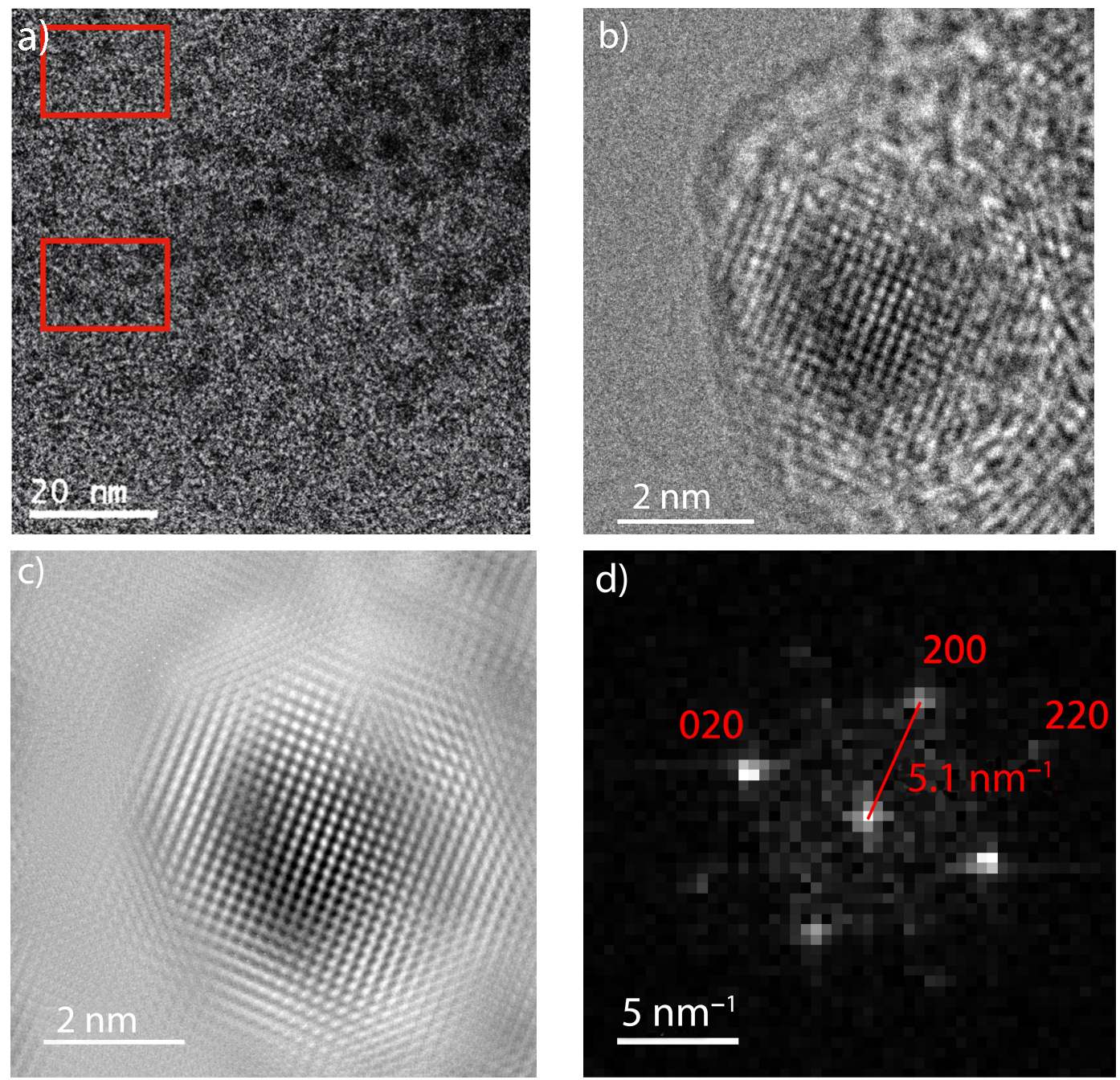

Figure 6-19 a) TEM image (FEI Titan 80-300 ETEM) of $\mathrm{Pd}_{24}\left(c-\mathbf{L}^{2}\right)_{48}$ obtained on a carboncoated $\mathrm{Cu}$ grid. Individual spherical particles with a size of 5-7 $\mathrm{nm}$ were observed. b) Enlarged view of one of the $\operatorname{Pd}(0)$ nanoparticles, which were observed after longer exposure under the electron beam. c) Figure 5-19b with Fourier filtering. d) Corresponding FT diffraction pattern of the $\mathrm{Pd}(0)$ nanoparticle along the [001] direction.

To obtain more evidence for the postulated large spherical assemblies, transmission electron microscopy (TEM) measurements were performed to visualize the size and shape of $\operatorname{Pd}_{24}\left(c-\mathbf{L}^{2}\right)_{48}$ rhombicuboctahedron. The samples were prepared on a perforated carboncoated copper grid by dampening the grid with acetonitrile solution of the complex

\footnotetext{
7 Transmission electron microscopy was measured with Patrick Perezki (IV. Physikalisches Institut, Göttingen).
} 
$\left[\mathrm{Pd}_{24}\left(c-\mathbf{L}^{2}\right)_{48}\right]\left(\mathrm{BF}_{4}\right)_{48 .}{ }^{[41]}$ The TEM images (Figure 6-19a) were quickly recorded after the solvent dried. Despite the very low contrast, particles having a diameter between 6-7 nm can be seen in the electron micrograph, which match the size of the rhombicuboctahedral model of $\operatorname{Pd}_{24}\left(c-\mathbf{L}^{2}\right)_{48}$ quite well. When the sample were subjected for longer times under the electron beam, however, sintering and formation of palladium(0) nanoparticles was observed (Figure 6-19b-c). The lattice constant of the nanoparticles is $3.92 \AA$, which fits very well with the lattice constant of the $f c c \operatorname{Pd}(0)$ crystalline structure.[42] This was observed probably because of the decomposition of the thin film formed by the dried solvent.

\subsubsection{Atomic force microscopy (AFM)}

Additional information about the size of $\operatorname{Pd}_{24}\left(c-\mathbf{L}^{2}\right)_{48}$ can be obtained by atomic force microscopy (AFM) experiments. Acetonitrile solution of $\operatorname{Pd}_{24}\left(c-\mathbf{L}^{2}\right)_{48}$ was deposited on a freshly cleaved graphite surface and subsequently dried in the air at room temperature.

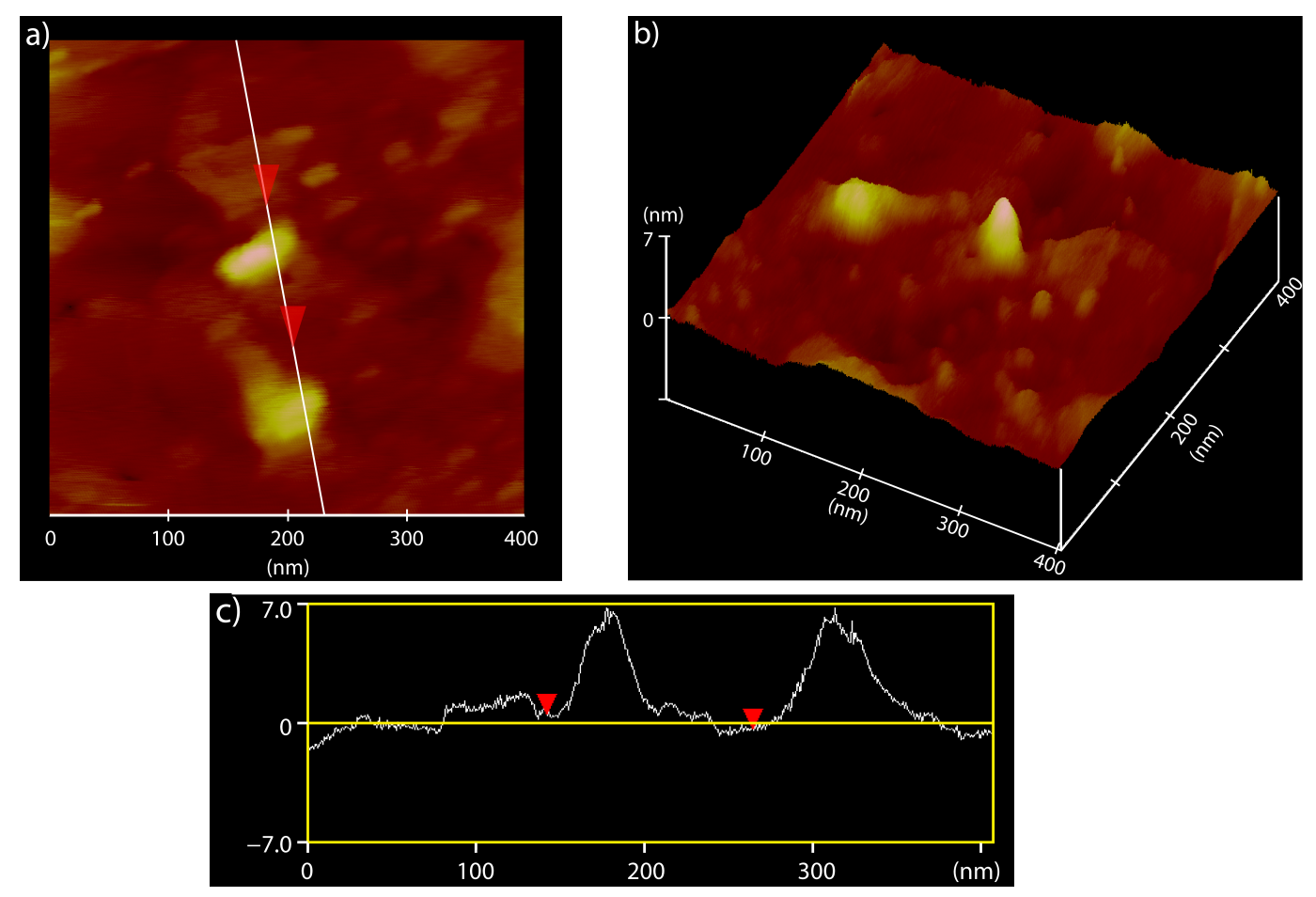

Figure 6-20 a) AFM image of $\operatorname{Pd}_{24}\left(c-\mathbf{L}^{2}\right)_{48}$ on graphite under dry conditions at room temperature. b) 3D image of Figure 6-21a. c) Height profiles of the selected AFM images for $\operatorname{Pd}_{24}\left(c-\mathbf{L}^{2}\right)_{48}($ NanoScope IV, Veeco).

The AFM images were captured by tapping mode, and showed spherical particles leading to a height above the surface of $6.8 \pm 0.5 \mathrm{~nm}$ (Figure 6-20). This result is consistent with the anticipated diameter of the large $\operatorname{Pd}_{24}\left(c \text { - } \mathbf{L}^{2}\right)_{48}$ self-assemblies as well. 


\subsubsection{Grazing-incidence small-angle X-ray scattering (GISAXS) ${ }^{8}$}

Furthermore, a grazing-incidence small-angle X-ray scattering (GISAXS) experiment was performed using a silicon substrate. It showed a diffuse scattering around $2 \theta=1.15^{\circ}$ caused by the correlation between the complex particles. The particle size could be extracted from the slope of the logarithmic intensity versus the square of $2 \theta$ ( $\mathrm{rad}$ ). According to Guinier approximation:[43]

$$
\ln I_{S A}(2 \theta)=\ln M n^{2} I_{e}-\frac{4}{3}\left(\frac{\pi}{\lambda}\right)^{2} R_{G}^{2}(2 \theta)^{2}
$$

with $M$ as the number of cage particles, $n$ as the number of the total electrons, $I_{e}$ as a single electron scattering intensity and $R_{G}$ as the Guinier radius, the size of the particles could be calculated. The value of of $R_{G}$ (around $2.9 \mathrm{~nm}$ ) could be evaluated from the slope of the curve, namely $5.8 \mathrm{~nm}$ in diameter (Figure 6-22). The particle size is slightly smaller than expected but still closer to the calculated diameter of the $\operatorname{Pd}_{24}\left(c-\mathbf{L}^{2}\right)_{48}$ sphere $(6.4 \mathrm{~nm})$ than that of the smaller $\operatorname{Pd}_{12}\left(c-\mathbf{L}^{2}\right)_{24}$ cage $(4.8 \mathrm{~nm}$ extracted from PM6 semiempirical molecular orbital method, Figure 6-9a).

a)

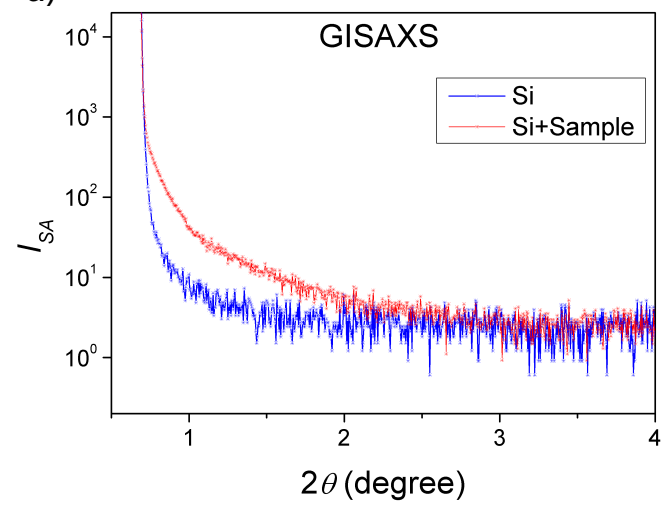

b)

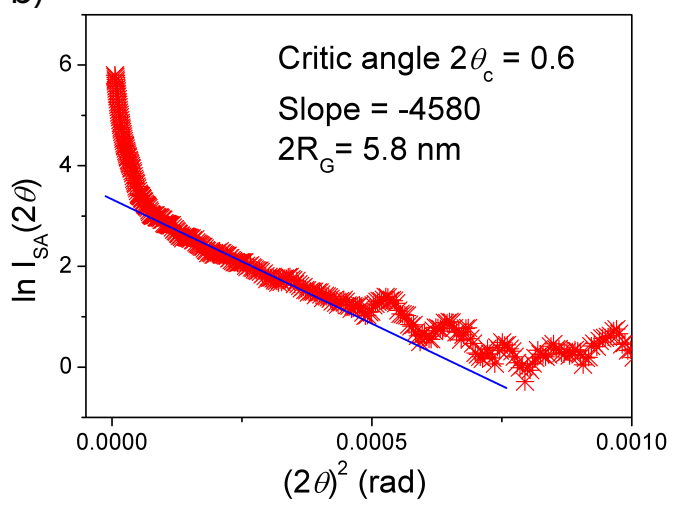

Figure 6-22 a) GISAXS data of the $\operatorname{Pd}_{12}\left(c-\mathbf{L}^{2}\right)_{24}$ film. The background intensities of $\mathrm{Si}$ substrate were shown in blue and the sample intensities on the Si substrate in red. b) The particle size of ca. $5.8 \mathrm{~nm}$ is extracted from the slope of the logarithmic intensity versus $(2 \theta)^{2}(\mathrm{rad})$ (Bruker D8 diffractometer).

8 Grazing-incidence small-angle X-ray scattering was measured with Dr. Yuansu Luo (I. Physikalisches Institut, Göttingen). 


\subsection{Kinetics of photoconversion}

First, the UV-Vis absorption spectra were measured of both $\mathbf{L}^{\mathbf{1}}$ (Figure 6-23a) and $\mathbf{L}^{2}$ system (Figure 6-23b). After irradiating the open-formed ligand or cage compounds, drastic changes could be observed in the UV-Vis spectra. Similar to the $\mathbf{L}^{\mathbf{1}}$ system (Figure 6-23a, Chapter 4 as well), the colourless $o$ - $\mathbf{L}^{2}$, which absorbs intensively at $300 \mathrm{~nm}$, can be photo-converted to the dark blue closed-form $c$ - $\mathbf{L}^{2}$ through irradiation with $313 \mathrm{~nm}$. New absorption bands appeared respectively at $\lambda=270,381$, and $584 \mathrm{~nm}$. One of the absorption maxima of the photo-generated $c-\mathbf{L}^{2}$ at $584 \mathrm{~nm}$ is a characteristic indicative band for ring-open and -closed interconversion. Conversely, irradiation with $617 \mathrm{~nm}$ on the ring-closed converts back the $c-\mathbf{L}^{2}$ to its open form (Figure 6-23b). The spectra of cages $\operatorname{Pd}_{3}\left(o-\mathbf{L}^{2}\right)_{6}$ and $\operatorname{Pd}_{24}\left(c-\mathbf{L}^{2}\right)_{48}$ show similar absorption bands as observed in $o-\mathbf{L}^{2}$ and $c$ - $\mathbf{L}^{2}$, respectively. However, the bands are shifted to longer wavelengths, which are ascribed to the coordination with palladium. The $\operatorname{Pd}_{3}\left(o-\mathbf{L}^{2}\right)_{6}$ presents a very intense absorption at $\lambda=323 \mathrm{~nm}$, and $\operatorname{Pd}_{24}\left(c-\mathbf{L}^{2}\right)_{48}$ with absorption maxima at 292, 397, and 621 $\mathrm{nm}$ (Figure 6-23b).
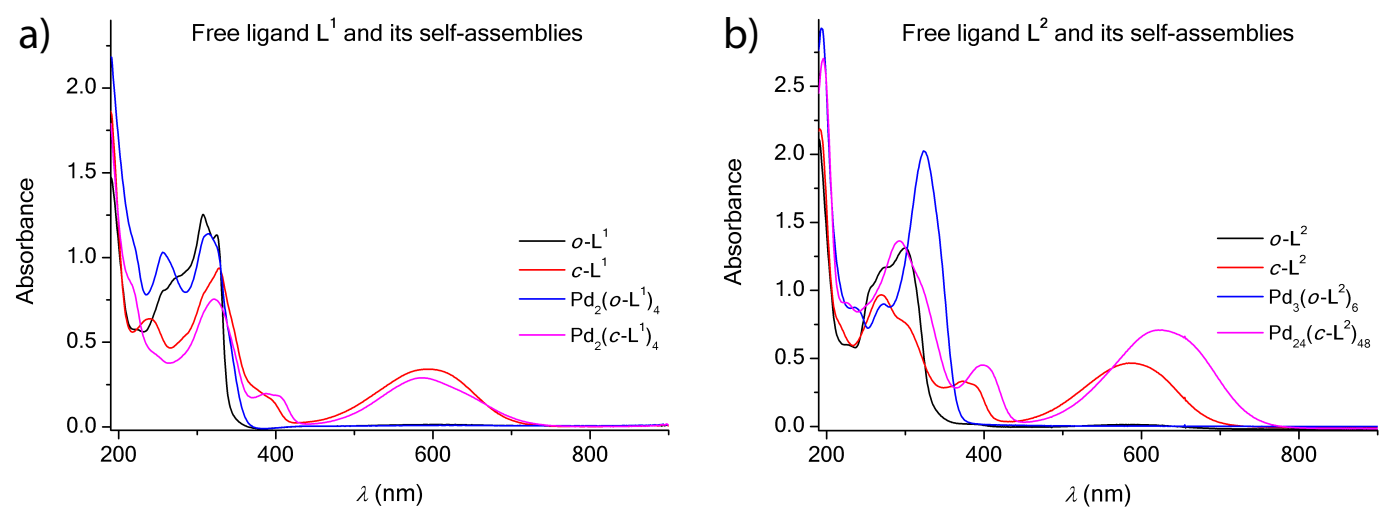

Figure 6-23 UV-Vis spectra of $\mathbf{L}^{1}, \mathbf{L}^{2}$ and their self-assemblies in both open and closed form. UV-Vis spectra of a) $o-\mathbf{L}^{\mathbf{1}}, c-\mathbf{L}^{\mathbf{1}}(0.125 \mathrm{mM}), \mathrm{Pd}_{2}\left(o-\mathbf{L}^{1}\right)_{4}, \mathrm{Pd}_{2}\left(c-\mathbf{L}^{1}\right)_{4}(0.031 \mathrm{mM})$ and b) $o-\mathbf{L}^{2}, c-\mathbf{L}^{2}(0.25 \mathrm{mM}), \mathrm{Pd}_{3}\left(o-\mathbf{L}^{2}\right)_{6}(0.042 \mathrm{mM}), \mathrm{Pd}_{24}\left(c-\mathbf{L}^{2}\right)_{48}(0.005 \mathrm{mM})$.

In order to examine the kinetics of the photoconversion of the previously reported ligand $\mathbf{L}^{1}$ and the new derivative $\mathbf{L}^{2}$ were examined, both as free ligands and as part of their palladium-mediated self-assemblies (Figure 6-24), a comparison experiment of the switching rates was carried out, using the same wavelengths and the same light sources for the photoreactions, which means $o$-L and $o$-C were irradiated upon $313 \mathrm{~nm}$ light, and $c$-L and $c$-C were irradiated upon $617 \mathrm{~nm}$ light. 
a)

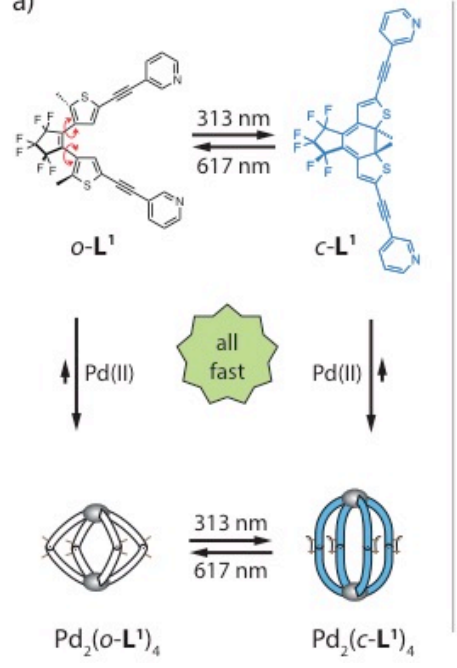

b)
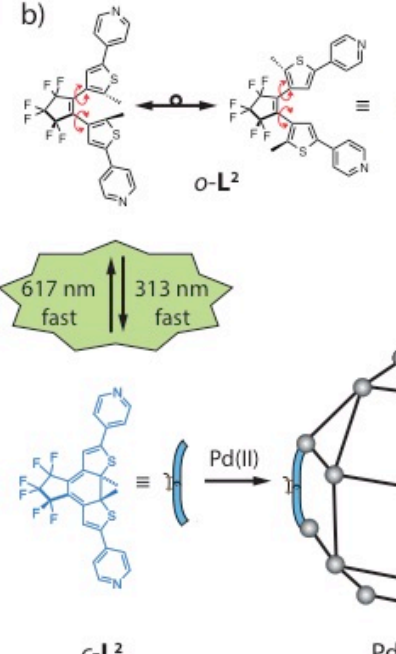

$c-L^{2}$ c)<smiles>C1CCCCC1</smiles>

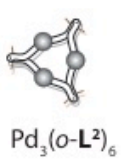

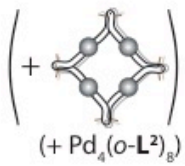

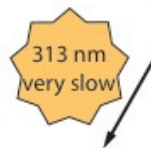

d)

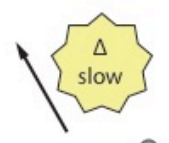

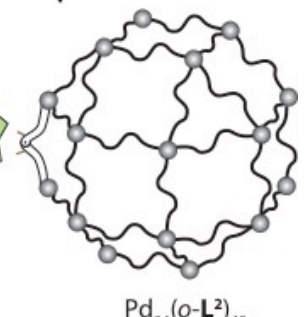

$\operatorname{Pd}_{24}\left(c-L^{2}\right)_{48}$

Figure 6-24 Comparison of photoswitching kinetics for a) the previously reported ligand $\mathbf{L}^{1}$ and its isomeric cages $\operatorname{Pd}_{2}\left(o-\mathbf{L}^{1}\right)_{4}$ and $\operatorname{Pd}_{2}\left(c-\mathbf{L}^{1}\right)_{4}$ with b) the new ligand $\mathbf{L}^{2}$ and the differently sized self-assemblies c) $\operatorname{Pd}_{3}\left(o-\mathbf{L}^{2}\right)_{6}$ and d) $\operatorname{Pd}_{24}\left(c-\mathbf{L}^{2}\right)_{48}$ based on this compound. Only in the latter case, Pd-pyridine bond breaking as well as conformational peculiarities of the photoswitch have a significant effect on the overall photoswitching kinetics. Copyright (C) 2016 WILEY-VCH Verlag GmbH \& Co.
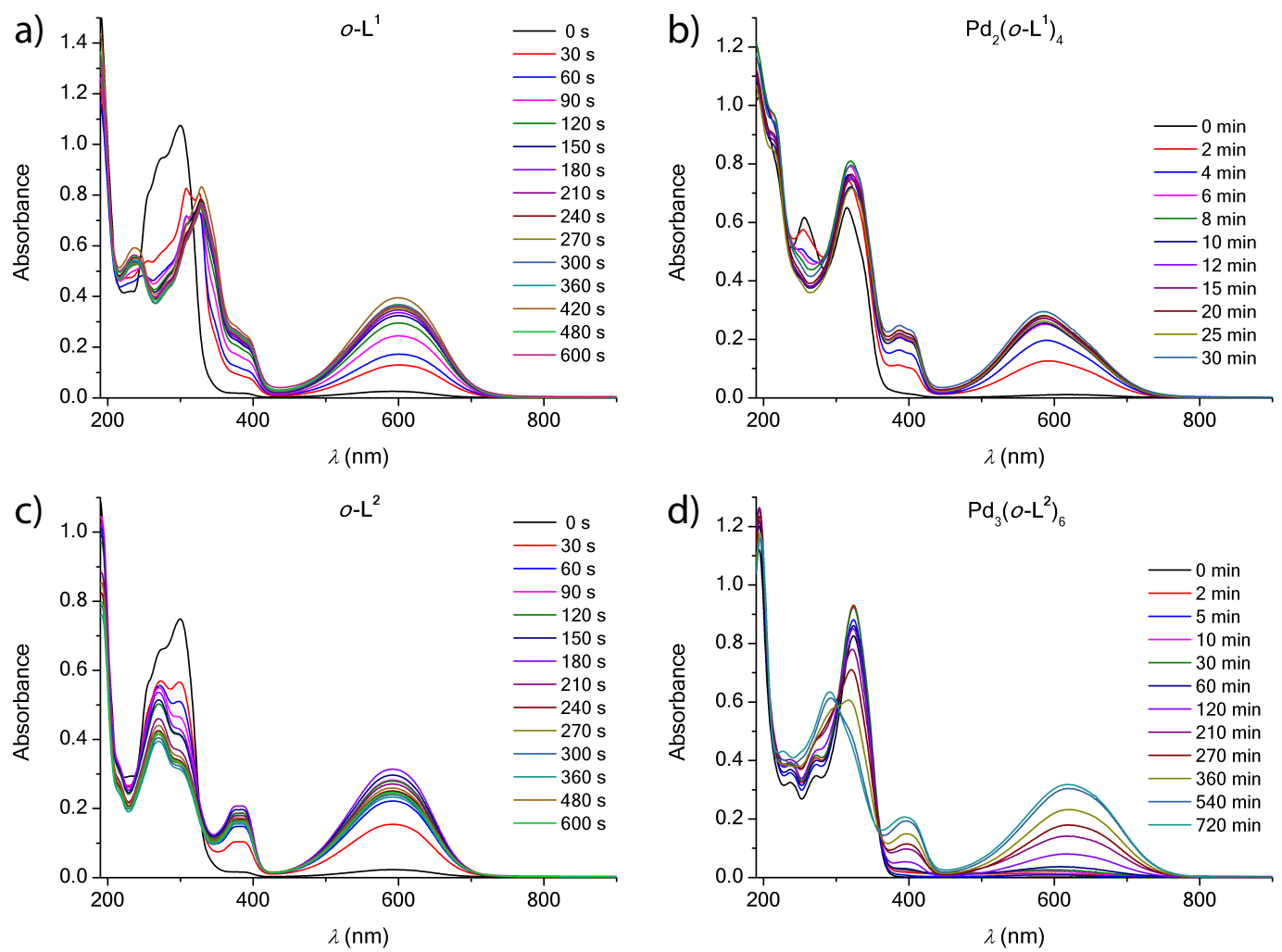

Figure 6-25 Time-dependent UV-Vis spectra of a) $o-\mathbf{L}^{\mathbf{1}}(0.125 \mathrm{mM})$, b) $\operatorname{Pd}_{2}\left(o-\mathbf{L}^{\mathbf{1}}\right)_{4}$ $(0.031 \mathrm{mM}), \mathrm{c}) o-\mathbf{L}^{2}(0.125 \mathrm{mM})$ and d) $\mathrm{Pd}_{3}\left(o-\mathbf{L}^{2}\right)_{6}(0.021 \mathrm{mM})$ irradiation at $313 \mathrm{~nm}$. 
The light-triggered interconversion between the assembled photoisomeric rings and cages was investigated by monitoring the time-dependent photoreaction with UV-Vis spectroscopy (Figure 6-25 and Figure 6-26). Besides, the formation of the rings and cages was confirmed by NMR spectroscopy, respectively.
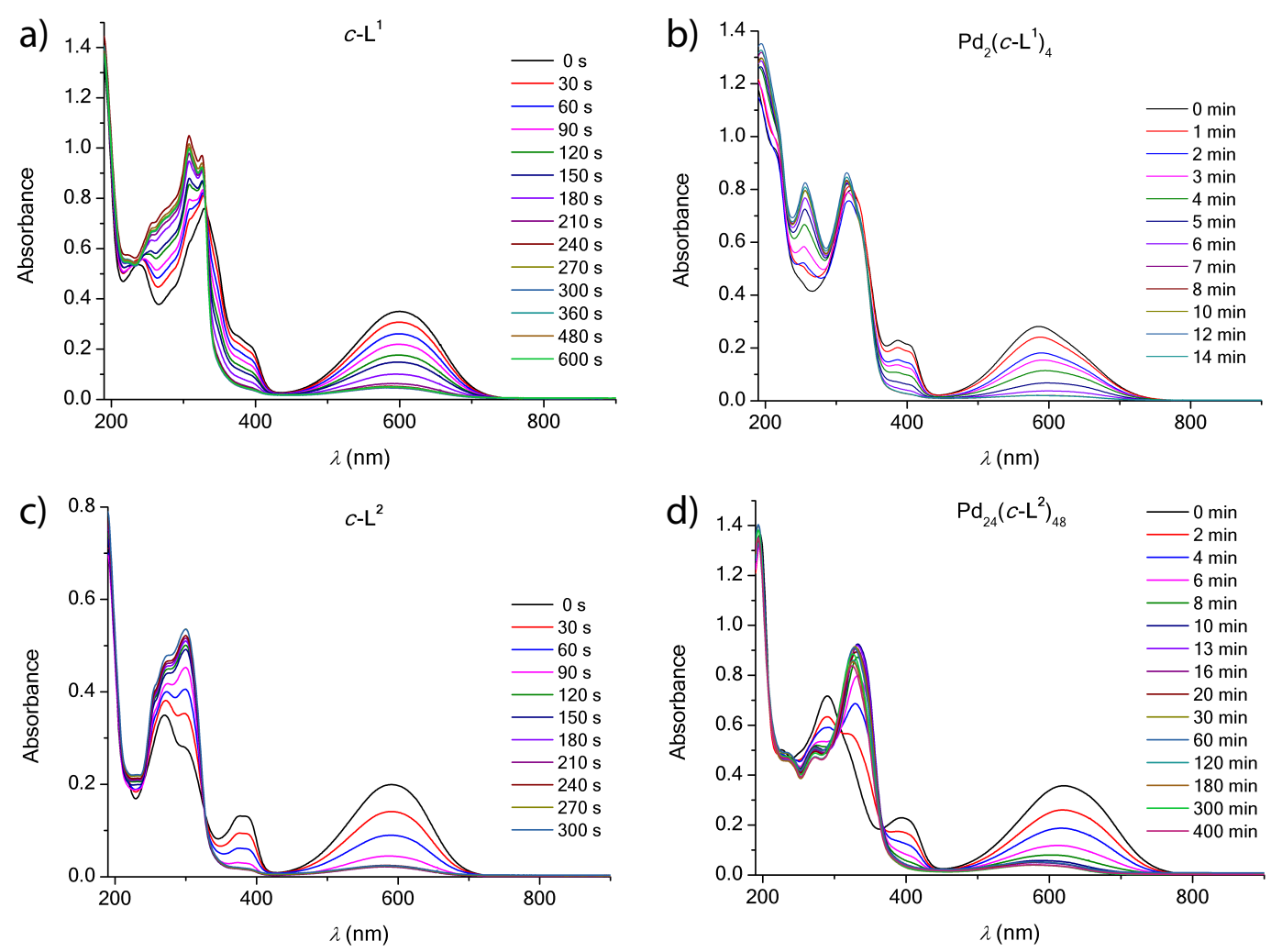

Figure 6-26 Time-dependent UV-Vis spectra of a) $c$ - $\mathbf{L}^{\mathbf{1}}(0.125 \mathrm{mM})$, b) $\operatorname{Pd}_{2}\left(c-\mathbf{L}^{\mathbf{1}}\right)_{4}$ $(0.031 \mathrm{mM}), c) c-\mathbf{L}^{2}(0.125 \mathrm{mM})$ and d) $\operatorname{Pd}_{24}\left(c-\mathbf{L}^{2}\right)_{48}(0.005 \mathrm{mM})$ irradiation at $617 \mathrm{~nm}$.

It revealed an interesting difference in the behaviour of the $\mathbf{L}^{\mathbf{1}}$ and $\mathbf{L}^{\mathbf{2}}$ systems: acetonitrile solutions of both free ligands $\mathbf{L}^{\mathbf{1}}$ and $\mathbf{L}^{\mathbf{2}}$ switch almost quantitatively back and forth within several minutes under the given conditions (Figure 6-27). However, the cage photoisomers present extremely different switching rates. The $\operatorname{Pd}_{2}\left(o-\mathbf{L}^{1}\right)_{4}$ and $\operatorname{Pd}_{2}\left(c-\mathbf{L}^{\mathbf{1}}\right)_{4}$ can be switched between each other reversibly in just a few minutes, which the time frame is similar to the free ligands. The same is true for the photoreaction carried out with the large spherical cage $\mathrm{Pd}_{24}\left(c-\mathbf{L}^{2}\right)_{48}$. However, when the $\mathrm{Pd}_{3}\left(o-\mathbf{L}^{2}\right)_{6}$ ring was irradiated with $313 \mathrm{~nm}$ UV light, the observed switching rate was extremely different (purple curve in Figure 6-27a) from that of spherical $\operatorname{Pd}_{24}\left(c-\mathbf{L}^{2}\right)_{48}$. 
a) Open to Closed

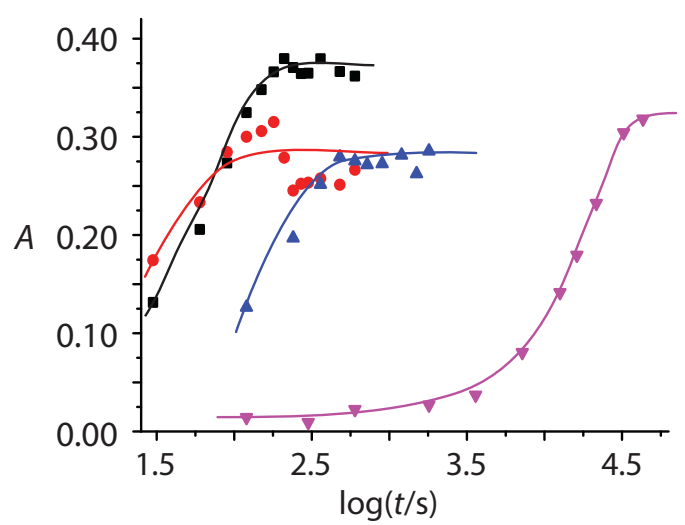

b)

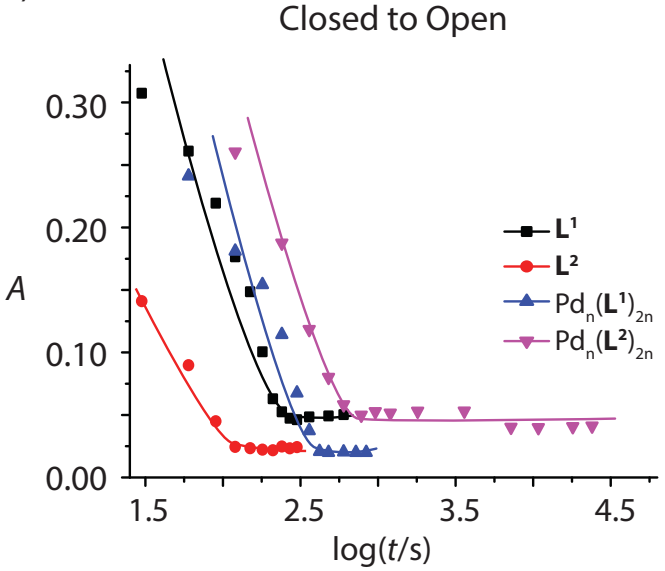

Figure 6-27 Kinetic comparison of the photoconversions of a) open-form ligands and cages under $313 \mathrm{~nm}$ irradiation, and b) closed-form ligands and cages upon $617 \mathrm{~nm}$ irradiation. Copyright (C) 2016 WILEY-VCH Verlag GmbH \& Co.

This observation can be explained through the different conformations in the discussed structures, which the open-form photoswitch adopts. The flexible open-form photoswitch can adopt several conformations, which differ in the effect of photoexciting the $\pi$-electron system. But the photocyclization reaction, which can occur only from the anti-parallel conformation, undergoes antarafacial, conrotatory electrocyclic ring closure in accordance with the Woodward-Hoffmann rules.[15] The open-form free ligands $o-\mathbf{L}^{1}$ and $o-\mathbf{L}^{2}$ as well as the cage $\operatorname{Pd}_{2}\left(\mathbf{L}^{1}\right)_{4}$ are readily able to adopt this conformation, thus leading to a relatively fast conversion in solution into the closed-form isomers (Figure 6-28a). In contrast, the formation of the ring compounds $\operatorname{Pd}_{3}\left(o-\mathbf{L}^{2}\right)_{6}$ and $\operatorname{Pd}_{4}\left(o-\mathbf{L}^{2}\right)_{8}$ requires the ligand backbones to adopt a highly twisted conformation, from which it is impossible to undergo the photocyclization because of the unfavorable orbital overlap (Figure 6-28b). Therefore, irradiation of the incorporated $o-\mathbf{L}^{2}$ leads to dissipation by radiationless relaxation processes and only the extremely small amounts of free $o-\mathbf{L}^{2}$ that are present in the equilibrium mixture of the $\mathrm{Pd}_{3}\left(o-\mathbf{L}^{2}\right)_{6}$ and $\mathrm{Pd}_{4}\left(o-\mathbf{L}^{2}\right)_{8}$ rings is able to undergo photocyclization. Under gradual but very slow shifting of the dynamic equilibrium, the photogenerated closed-form ligand $c-\mathbf{L}^{2}$ then coordinates with $\mathrm{Pd}$ II cations and form the $\operatorname{Pd}_{24}\left(c-\mathbf{L}^{2}\right)_{48}$ sphere. In order to achieve the completeness of photocyclization and the clean formation of the large spheres, an irradiation process that was measured to take about $15 \mathrm{~h}$ before the condition is reached. 
a)

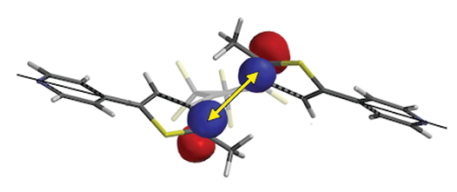

$\Varangle 148.6^{\circ}$ b)

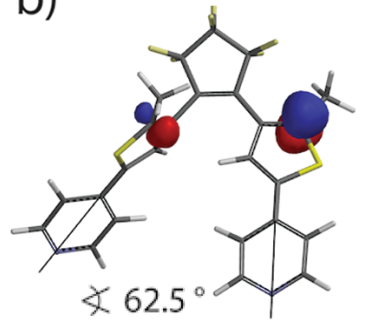

c)

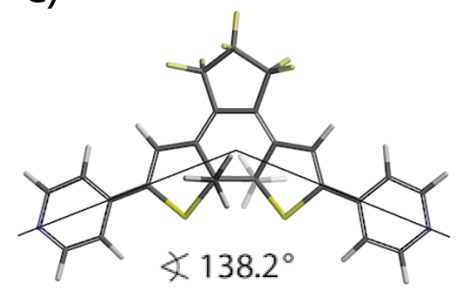

Figure 6-28 Conformation of ligand $o-\mathbf{L}^{2}$ a) in the free state or as a part of the sphere $\operatorname{Pd}_{24}\left(\mathbf{L}^{2}\right)_{48}$ and b) as a part of the ring $\operatorname{Pd}_{3}\left(o-\mathbf{L}^{2}\right)_{6}$. The frontier orbitals whose constructive overlap is required for the photocyclization are indicated. c) Geometry-optimized structure of closed-form ligand $c$ - $\mathbf{L}^{2}$. Copyright (C) 2016 WILEY-VCH Verlag GmbH \& Co.

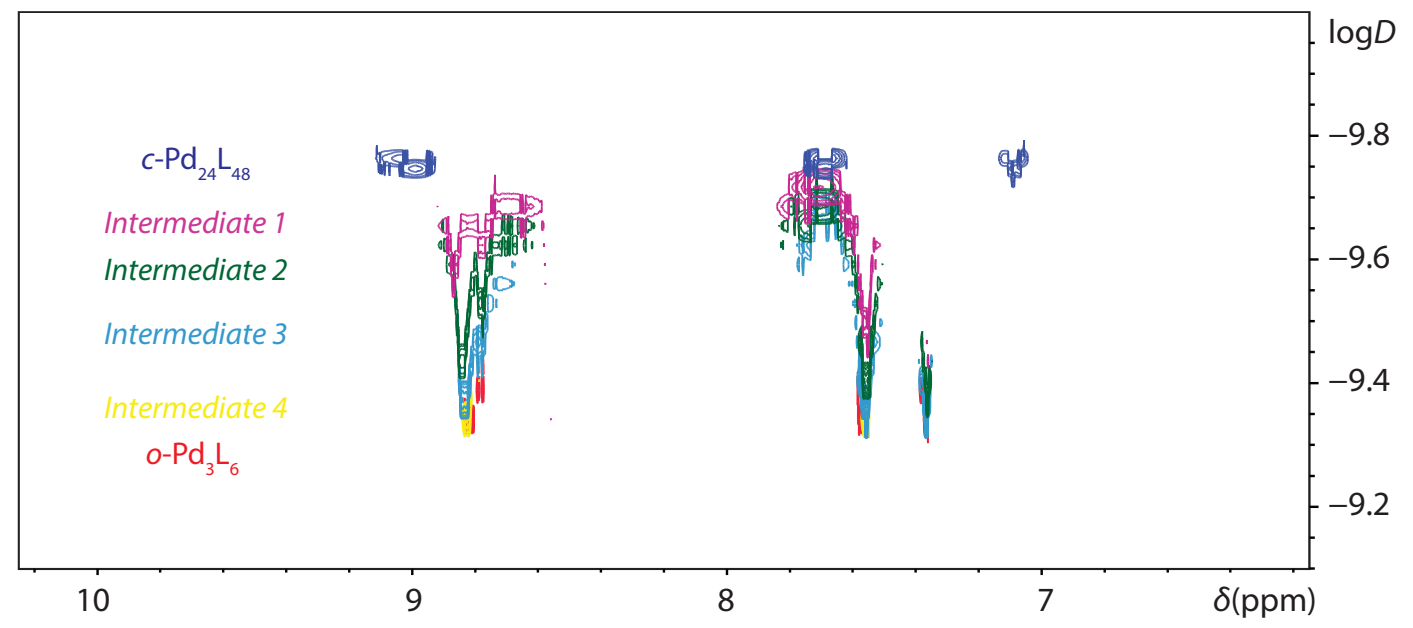

Figure 6-29 Superposition of time-dependent ${ }^{1} \mathrm{H}$-DOSY NMR spectra (500 $\mathrm{MHz}, \mathrm{CD}_{3} \mathrm{CN}$ ) of intermediate complexes. Spectra were recorded immediately after the $\operatorname{Pd}_{24}\left(c-\mathbf{L}^{2}\right)_{48}$ sample was irradiated with $617 \mathrm{~nm}$ light for $10 \mathrm{~min}$, and store at $298 \mathrm{~K}$ for $1 \mathrm{~h}$ (Intermediate 1), $7 \mathrm{~h}$ (Intermediate 2), $42 \mathrm{~h}$ (Intermediate 3), $48 \mathrm{~h}$ at $298 \mathrm{~K}+16 \mathrm{~h}$ at $323 \mathrm{~K}$ (Intermediate 4). DOSY spectra of the samples containing $\operatorname{Pd}_{3}\left(o-\mathbf{L}^{2}\right)_{6}+\operatorname{Pd}_{4}\left(o-\mathbf{L}^{2}\right)_{8}$ and the sphere $\operatorname{Pd}_{24}\left(c-\mathbf{L}^{2}\right)_{48}$ $\left(400 \mathrm{MHz}, \mathrm{CD}_{3} \mathrm{CN}\right.$ ) were superimposed as comparison.

Furthermore, the reverse photoreaction of the $\operatorname{Pd}_{24}\left(c-\mathbf{L}^{2}\right)_{48}$ sphere to form the three- and four rings requires decomplexation and recomplexation of the ligands to the $\operatorname{Pd}(\mathrm{II})$ cations as well. But it has to be noted that the ring-opening photochromic reaction happens first and is quickly finished, due to the favourable preorientated orbital overlap of the $c-\mathbf{L}^{2}$, then generate a tentative open-form $\operatorname{Pd}_{24}\left(o-\mathbf{L}^{2}\right)_{48}$ sphere subsequently (Figure 6-24d). However, this open-form intermediate $\operatorname{Pd}_{24}\left(o-L^{2}\right)_{48}$ is not the thermodynamic minimum of the system, thus it breaks apart and yield the entropically favoured small three- and fourring assemblies. In order to track the disintegration process of the unstable sphere 
$\operatorname{Pd}_{24}\left(o-\mathbf{L}^{2}\right)_{48}$, time-dependent ${ }^{1} \mathrm{H}$-DOSY experiments were carried out. A sample of $\operatorname{Pd}_{24}\left(c-\mathbf{L}^{2}\right)_{48}$ was first irradiated with $617 \mathrm{~nm}$ light for $10 \mathrm{~min}$ until the ring-opening reactions of all photoswitches had been finished and then was subjected immediately to a consecutive series of DOSY measurements. As depicted in Figure 6-29, a gradual decay of the large structure proceeding via a series of intermediate-sized objects to yield the small $\operatorname{Pd}_{3}\left(o-\mathbf{L}^{2}\right)_{6}$ and $\operatorname{Pd}_{4}\left(o-\mathbf{L}^{2}\right)_{8}$ rings was observed in the spectra. The rearrangement was found to be temperature dependent. At $50{ }^{\circ} \mathrm{C}$, it took 16 hours to complete, at $70{ }^{\circ} \mathrm{C}$, it was completed within 5 min.

The kinetic differences of the light-triggered reactions in the examined systems answer the previously raised question whether the photoisomerization of cage $\operatorname{Pd}_{2}\left(\mathbf{L}^{1}\right)_{4}$ follows the route: cage decomplexation, photoswitching of the free ligand, recoordinate to the metal or if the integrity of the cage is maintained during the switching. In comparison with the present system, we can now assume that the latter situation serves as the best mechanistic explanation for the switching in that cage.

\subsection{Conclusion}

In this chapter, a complete structural rearrangement between self-assemblies of different nuclearities based on the implementation of light-switchable backbones into palladiummediated structures was achieved. The open-form ligand $o-\mathbf{L}^{2}$ leads to the formation of small three- or four-membered rings upon complexation with $\mathrm{Pd}(\mathrm{II})$ cations, whereas the closed-form ligand $o-\mathbf{L}^{2}$ assembles a large spherical $\operatorname{Pd}_{24}\left(c-\mathbf{L}^{2}\right)_{48}$ rhombicuboctahedron about $6.4 \mathrm{~nm}$ in diameter containing 2712 atoms. The sphere could be easily transformed into the small rings upon short-time irradiation, but the reverse reaction was found to be extremely slow. This dithienylethene photoswitch integrated stimuli-responsive structural reorganization processes with the $\mathrm{Pd}(\text { pyridine })_{4}$ coordination motif will significantly contribute to the development of a next generation of artificial self-assembled systems that joins light-triggered elements with a variety of other functionalities. The control over such structural rearrangements promises to promote development in the fields such as supramolecular catalysis, molecular machinery, nanomedicine and molecular electronics. 


\subsection{Experimental section}

\subsubsection{Synthesis of the cage compounds}

\subsubsection{Synthesis of ligand $o-\mathbf{L}^{2}$}
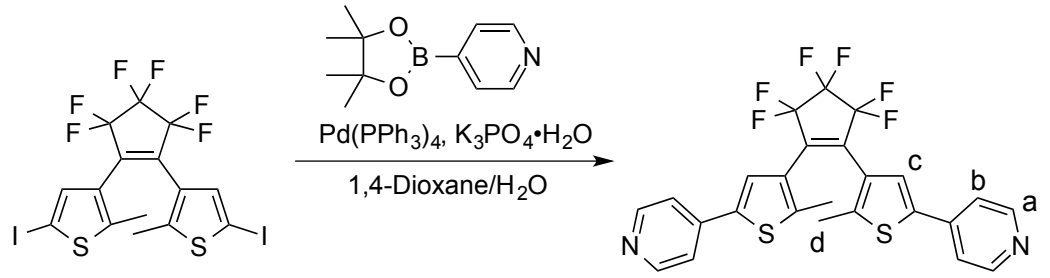

A mixture of $\mathrm{Pd}\left(\mathrm{PPh}_{3}\right)_{4}$ (48.1 mg, $0.05 \mathrm{mmol}, 10.5 \mathrm{~mol} \%$ ), 4-pyridineboronic acid pinacol ester (316 mg, $1.54 \mathrm{mmol}, 3.2$ equiv.), $\mathrm{K}_{3} \mathrm{PO}_{4} \cdot \mathrm{H}_{2} \mathrm{O}$ (1.78 g, $7.70 \mathrm{mmol}, 16$ equiv.), perfluoro1,2-bis(2-iodo-5-methylthien-4-yl)cyclopentene ${ }^{[44][45]}$ (300 mg, $0.48 \mathrm{mmol}, 1$ equiv.) and degassed distilled water (6 mL) and 1,4-dioxane (12 mL) was combined in an oven-dried Schlenk tube and stirred at $90{ }^{\circ} \mathrm{C}$ overnight. The mixture was monitored by TLC. Then the reaction mixture was allowed to cool to room temperature, concentrated under reduced pressure, washed with $\mathrm{CHCl}_{3}$ and $\mathrm{H}_{2} \mathrm{O}$, dried over anhydrous $\mathrm{MgSO}_{4}$ and concentrated in vacuo. The crude product was purified by column chromatography (silica) using $\mathrm{CHCl}_{3} / \mathrm{MeOH}$ (gradient) as eluents to give $o-\mathbf{L}^{2}(197.3 \mathrm{mg}, 78 \%)$ as a pale yellow solid.

1H-NMR (300 MHz, CD 3 CN) $\delta 2.04\left(6 \mathrm{H}, \mathrm{s}, \mathrm{H}_{\mathrm{d}}\right), 7.51\left(4 \mathrm{H}, \mathrm{dd},{ }^{3} \mathrm{~J}=6.0 \mathrm{~Hz},{ }^{4}=1.5 \mathrm{~Hz}, \mathrm{H}_{\mathrm{b}}\right), 7.62$ $\left(2 \mathrm{H}, \mathrm{s}, \mathrm{H}_{\mathrm{c}}\right), 8.57\left(4 \mathrm{H}, \mathrm{d}, 3 \mathrm{~J}=6.0 \mathrm{~Hz}, \mathrm{H}_{\mathrm{a}}\right)$.

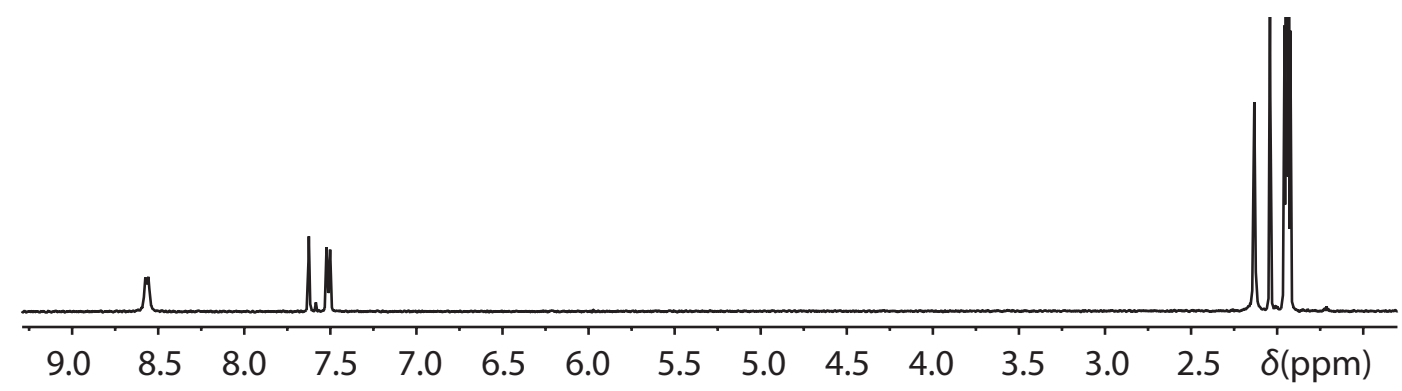

${ }^{13}$ C-NMR (75 MHz, CD $\left.{ }_{3} \mathrm{CN}\right) \delta 14.87,120.40,126.26,126.63,140.21,140.79,145.17,151.47$.

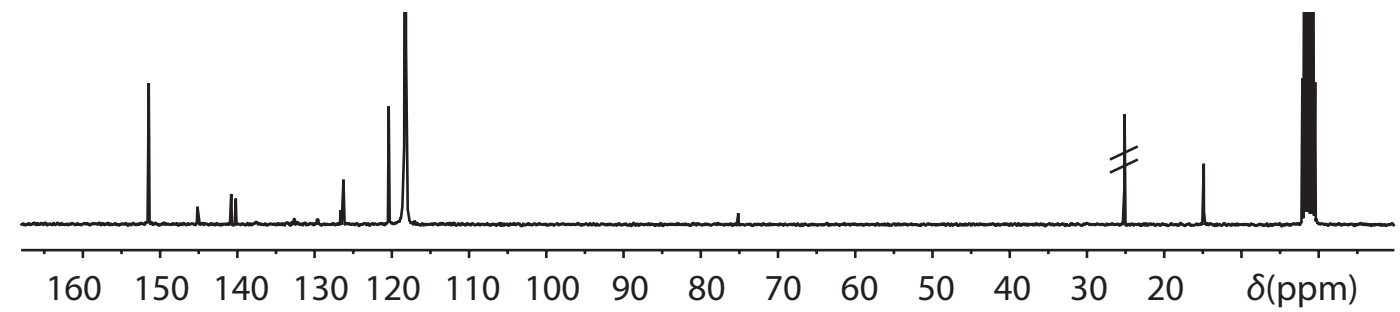


19F-NMR $\left(282 \mathrm{MHz}, \mathrm{CD}_{3} \mathrm{CN}\right) \delta-110.86\left(4 \mathrm{~F}, \mathrm{t},{ }^{3} \mathrm{~J}=5.1 \mathrm{~Hz}\right),-132.54\left(2 \mathrm{~F}\right.$, quint, $\left.{ }^{3} \mathrm{~J}=5.1 \mathrm{~Hz}\right)$.

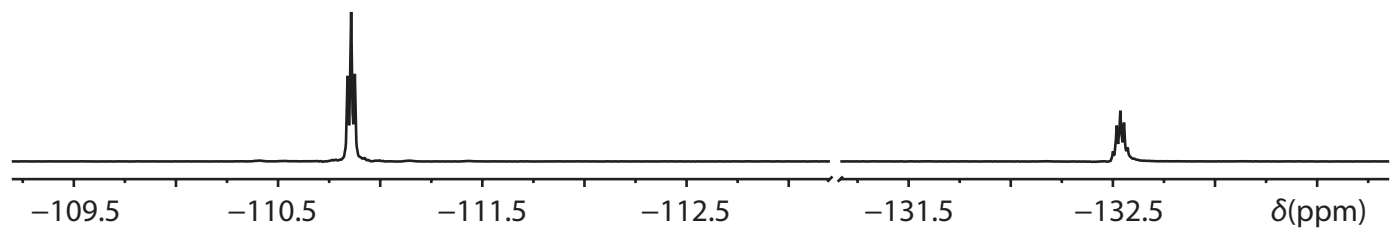

ESI-MS (pos.) $m / z=523.1\left[o-\mathbf{L}^{2}+\mathrm{H}\right]^{+}$.

\subsubsection{Synthesis of ring and cage compounds}

The open-form ring compound was synthesized by heating a mixture of the ligand $o-\mathbf{L}^{2}$ $\left(12 \mu \mathrm{mol}, 400 \mu \mathrm{L}\right.$ of a $30 \mathrm{mM}$ stock solution in $\left.\mathrm{CD}_{3} \mathrm{CN}\right)$ and $\left[\mathrm{Pd}\left(\mathrm{CH}_{3} \mathrm{CN}\right)_{4}\right]\left(\mathrm{BF}_{4}\right)_{2}(6 \mu \mathrm{mol}$, $200 \mu \mathrm{L}$ of a $30 \mathrm{mM}$ stock solution in $\mathrm{CD}_{3} \mathrm{CN}$ ) in $2400 \mu \mathrm{L} \mathrm{CD}{ }_{3} \mathrm{CN}$ at $70{ }^{\circ} \mathrm{C}$ for $1 \mathrm{~h}$ in a closed vial to yield $3000 \mu \mathrm{l}$ of a $0.67 \mathrm{mM}$ solution containing the rings $\left[\mathrm{Pd}_{3}\left(o-\mathbf{L}^{2}\right)_{6}\right]\left(\mathrm{BF}_{4}\right)_{6}$ and $\left[\mathrm{Pd}_{4}\left(o-\mathbf{L}^{2}\right)_{8}\right]\left(\mathrm{BF}_{4}\right)_{8}$ in a ratio of 3:1 (Figure 6-4).

1H-NMR $\left(400 \mathrm{MHz}, \mathrm{CD}_{3} \mathrm{CN}\right) \delta 2.36\left(36 \mathrm{H}, \mathrm{s}, \mathrm{H}_{\mathrm{d}}\right), 7.40\left(12 \mathrm{H}, \mathrm{s}, \mathrm{H}_{\mathrm{c}}\right), 7.59(24 \mathrm{H}, \mathrm{dd}, 3 \mathrm{~J}=6.8 \mathrm{~Hz}$, $\left.{ }^{4} J=1.2 \mathrm{~Hz}, \mathrm{H}_{\mathrm{b}}\right), 8.57\left(24 \mathrm{H}, \mathrm{d}, 3 J=6.8 \mathrm{~Hz}, \mathrm{H}_{\mathrm{a}}\right)\left(\mathrm{Pd}_{3}\left(o-\mathrm{L}^{2}\right)_{6}\right.$, red in Figure 6-4a); $2.36(48 \mathrm{H}, \mathrm{s}$, $\mathrm{H}_{\mathrm{d}}$ ), $7.41\left(16 \mathrm{H}, \mathrm{s}, \mathrm{H}_{\mathrm{c}}\right), 7.61\left(32 \mathrm{H}, \mathrm{m}, \mathrm{H}_{\mathrm{b}}\right), 8.82\left(32 \mathrm{H}, \mathrm{d}, 3 \mathrm{~J}=6.8 \mathrm{~Hz}, \mathrm{H}_{\mathrm{a}}\right.$ ) (signals of minor species $\mathrm{Pd}_{4}\left(o-\mathbf{L}^{2}\right)_{8}$, green in Figure 6-4a).

${ }^{13}$ C-NMR $\left(125 \mathrm{MHz}, \mathrm{CD}_{3} \mathrm{CN}\right) \delta 14.96,123.82,126.90,131.05,136.74,138.84,145.45$, 148.61, $152.36\left(\operatorname{Pd}_{3}\left(o-\mathbf{L}^{2}\right)_{6}\right.$, red in Figure 6-4b); 15.11, 123.67, 127.01, 130.78, 136.77, $138.64,145.24,148.56,152.08$ (signals of minor species $\operatorname{Pd}_{4}\left(o-\mathbf{L}^{2}\right)_{8}$, green boxes in Figure 6-4b).

HRMS (ESI, pos.) calculated $\quad\left[\mathrm{Pd}_{3}\left(o-\mathrm{L}^{2}\right)_{6}+2\left(\mathrm{BF}_{4}\right)\right]^{4+} \mathrm{m} / z=906.7774$ found 906.7715;

$$
\left[\mathrm{Pd}_{4}\left(o-\mathbf{L}^{2}\right)_{8}+3\left(\mathrm{BF}_{4}\right)\right]^{5+} \mathrm{m} / z=973.2312 \text { found } 973.2213
$$

The closed cage compound was synthesized in quantitative yield by heating a mixture of the ligand $c-\mathbf{L}^{2}\left(12 \mu \mathrm{mol}, 400 \mu \mathrm{L}\right.$ of a $30 \mu \mathrm{mM}$ stock solution in $\left.\mathrm{CD}_{3} \mathrm{CN}\right)$ and $\left[\mathrm{Pd}\left(\mathrm{CH}_{3} \mathrm{CN}\right)_{4}\right]\left(\mathrm{BF}_{4}\right)_{2}\left(6 \mu \mathrm{mol}, 200 \mu \mathrm{L}\right.$ of a $30 \mathrm{mM}$ stock solution in $\left.\mathrm{CD}_{3} \mathrm{CN}\right)$ in $2400 \mu \mathrm{L}$ $\mathrm{CD}_{3} \mathrm{CN}$ at $70{ }^{\circ} \mathrm{C}$ for $1 \mathrm{~h}$ in a closed vial to yield $3000 \mu \mathrm{l}$ of a $83.3 \mu \mathrm{M}$ solution of the cage compound $\left[\mathrm{Pd}_{24}\left(c-\mathbf{L}^{2}\right)_{48}\right]\left(\mathrm{BF}_{4}\right)_{48}$.

${ }^{1}$ H-NMR $\left(300 \mathrm{MHz}, \mathrm{CD}_{3} \mathrm{CN}\right) \delta 1.98-2.06\left(288 \mathrm{H}, \mathrm{m}, \mathrm{H}_{\mathrm{d}}\right), 6.99-7.18\left(96 \mathrm{H}, \mathrm{m}, \mathrm{H}_{\mathrm{c}}\right), 7.58-7.81$ $\left(96 \mathrm{H}, \mathrm{m}, \mathrm{H}_{\mathrm{b}}\right), 8.84-9.18\left(96 \mathrm{H}, \mathrm{m}, \mathrm{H}_{\mathrm{a}}\right)$. 


\subsubsection{Synthesis of the three-membered ring with nitrate as counter anion}

The $\left[\mathrm{Pd}_{3}\left(o-\mathbf{L}^{2}\right)_{6}\right]\left(\mathrm{NO}_{3}\right)_{6}$ ring was synthesized as a single product in quantitative yield by heating a mixture of the ligand $o-\mathrm{L}^{2}\left(2.4 \mu \mathrm{mol}, 80 \mu \mathrm{L}\right.$ of a $30 \mathrm{mM}$ stock solution in $\left.\mathrm{CD}_{3} \mathrm{CN}\right)$ and $\mathrm{Pd}\left(\mathrm{NO}_{3}\right)_{2} \cdot \mathrm{H}_{2} \mathrm{O}\left(1.2 \mu \mathrm{mol}, 40 \mu \mathrm{L}\right.$ of a $30 \mathrm{mM}$ stock solution in $\left.\mathrm{CD}_{3} \mathrm{CN}\right)$ in $480 \mu \mathrm{L} \mathrm{CD}{ }_{3} \mathrm{CN}$ at $70{ }^{\circ} \mathrm{C}$ for $1 \mathrm{~h}$ in a closed vial to yield $600 \mu \mathrm{L}$ of a $0.67 \mathrm{mM}$ solution of the ring compound (Figure 6-6). After irradiation at $313 \mathrm{~nm}$, however, all NMR signals of the supramolecular structure vanished, due to the formation of a precipitate. Therefore, further experiments such as irradiation behaviour and kinetics were carried out using $\mathrm{BF}_{4}^{-}$as the counter anion.

1H-NMR (300 MHz, CD $\left.{ }_{3} \mathrm{CN}\right) \delta 2.31\left(36 \mathrm{H}, \mathrm{s}, \mathrm{H}_{\mathrm{d}}\right), 7.32\left(12 \mathrm{H}, \mathrm{s}, \mathrm{H}_{\mathrm{c}}\right), 7.58(24 \mathrm{H}, \mathrm{dd}, J=6.9,1.5$ $\left.\mathrm{Hz}, \mathrm{H}_{\mathrm{b}}\right), 9.24\left(24 \mathrm{H}, \mathrm{dd}, J=6.9,1.5 \mathrm{~Hz}, \mathrm{H}_{\mathrm{a}}\right)$.

HRMS (ESI, pos.) calculated $\quad\left[\mathrm{Pd}_{3}\left(o-\mathbf{L}^{2}\right)_{6}+2\left(\mathrm{NO}_{3}\right)\right]^{4+} \mathrm{m} / z=894.5211$ found 894.5227.

\subsubsection{Photoswitching between the ligand and ring/cage isomers}

In front of Irradiations at $313 \mathrm{~nm}$ were performed by placing a quartz cuvette or NMR tube in a distance of $2 \mathrm{~cm}$ in front of a LOT-Oriel $500 \mathrm{~W}$ mercury pressure lamp equipped with a dichroitic mirror and a $313 \mathrm{~nm}$ bandpass filter. Irraditaion at $617 \mathrm{~nm}$ were performed by placing a quartz NMR tube or cuvette in a distance of $2.5 \mathrm{~cm}$ in front of a power LED irradiation apparatus by Sahlmann photonics. The whole processes were monitored by NMR spectroscopy (Figure 6-30).

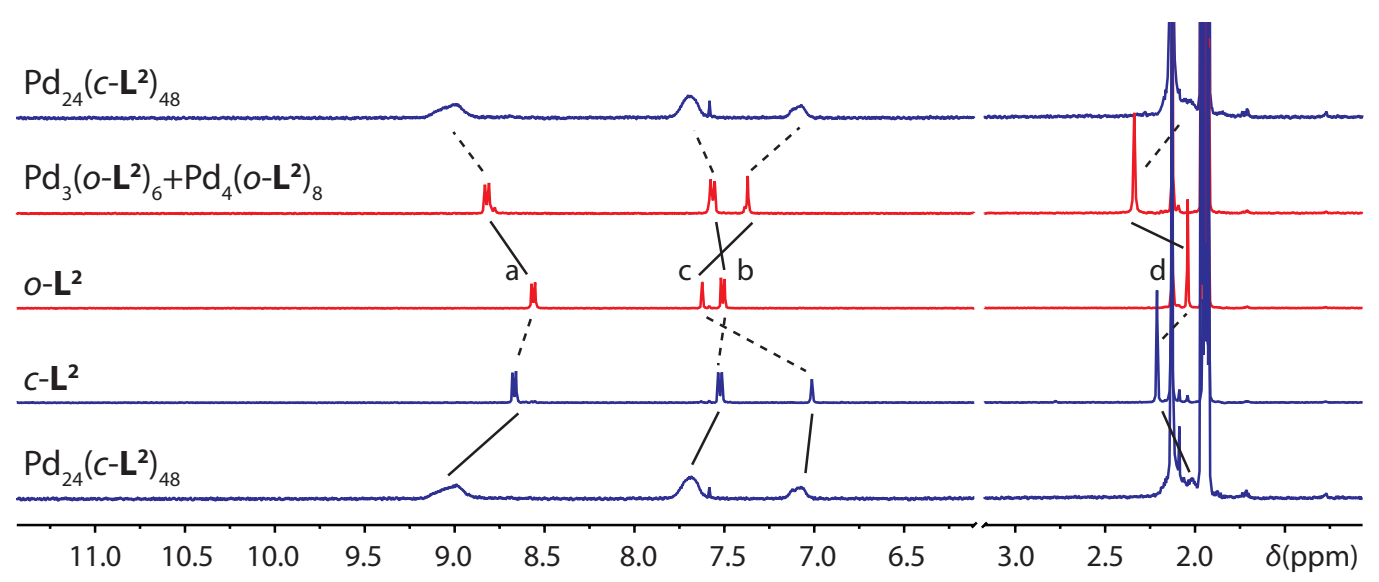

Figure 6-30 ${ }^{1} \mathrm{H}$ NMR spectra $\left(300 \mathrm{MHz}, \mathrm{CD}_{3} \mathrm{CN}\right)$ showing the interconversion between $o-\mathbf{L}^{2}, c-\mathbf{L}^{2}, \mathrm{Pd}_{3}\left(o-\mathbf{L}^{2}\right)_{6}+\mathrm{Pd}_{4}\left(o-\mathbf{L}^{2}\right)_{8}$ and $\mathrm{Pd}_{24}\left(c-\mathbf{L}^{2}\right)_{48}$ including the aliphatic region. 


\subsubsection{DOSY NMR spectra of the rings and cages}

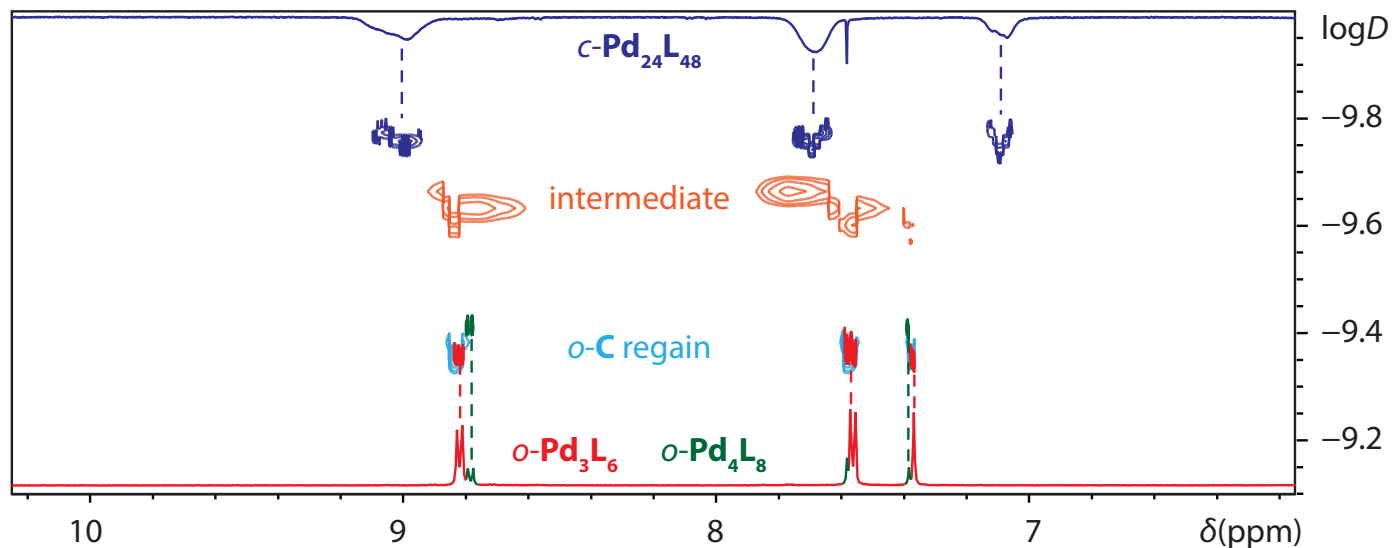

Figure 6-31 Superposition of DOSY NMR spectra (400 $\left.\mathrm{MHz}, \mathrm{CD}_{3} \mathrm{CN}\right)$ of cage complexes $\mathrm{Pd}_{3}\left(o-\mathbf{L}^{2}\right)_{6}+\mathrm{Pd}_{4}\left(o-\mathbf{L}^{2}\right)_{8}, \mathrm{Pd}_{24}\left(c-\mathbf{L}^{2}\right)_{48}$, an intermediate in the disassembly pathway (with unknown nuclearity) obtained after 10 min irradiation of $\operatorname{Pd}_{24}\left(c-\mathbf{L}^{2}\right)_{48}$ under $617 \mathrm{~nm}$, and the regained rings $\operatorname{Pd}_{3}\left(o-\mathbf{L}^{2}\right)_{6}+\mathrm{Pd}_{4}\left(o-\mathbf{L}^{2}\right)_{8}$ by heating the irradiated solution of $\operatorname{Pd}_{24}\left(\mathbf{L}^{2}\right)_{48}$ for $5 \min$ at $70^{\circ} \mathrm{C}$.

Hydrodynamic radii were calculated from the diffusion coefficient values using the Stokes-Einstein equation (Figure 6-31):

$$
r=\frac{k \cdot T}{6 \cdot \pi \cdot \eta \cdot D}
$$

Table 6-1 Calculated hydrodynamic radii for ring/cage complexes and intermediate.

\begin{tabular}{|c|c|c|c|c|}
\hline complex & $\mathrm{Pd}_{3}\left(o-\mathrm{L}^{2}\right)_{6}$ & $\mathrm{Pd}_{4}\left(o-\mathrm{L}^{2}\right)_{8}$ & $\mathrm{Pd}_{24}\left(c-\mathrm{L}^{2}\right)_{48}$ & intermediate \\
\hline $\log D$ & -9.36 & -9.41 & -9.78 & -9.64 \\
\hline$D\left[\times 10^{-10} \mathrm{~m}^{2} \mathrm{~s}^{-1}\right]$ & 4.33 & 3.86 & 1.67 & 2.27 \\
\hline$r[\mathrm{~nm}]$ & 1.36 & 1.52 & 3.52 & 2.61 \\
\hline
\end{tabular}

\subsubsection{Chiral separation of $c-\mathbf{L}^{2}$}

The open-form ligand $o-\mathbf{L}^{2}(20 \mathrm{mg})$ was taken up in $\mathrm{CD}_{2} \mathrm{Cl}_{2}(2 \mathrm{~mL})$ and was irradiated with $313 \mathrm{~nm}$ UV light. The success of the irradiation was followed by ${ }^{1} \mathrm{H}$ NMR spectroscopy. The sample was then concentrated to $1 \mathrm{~mL}$, filtered and purified by preparative chiral HPLC using a Daicel IA column under strict exclusion of daylight. The purity of separated $(R, R)$ and $(S, S)$ enantiomers was checked using analytical HPLC (Figure 6-32). 

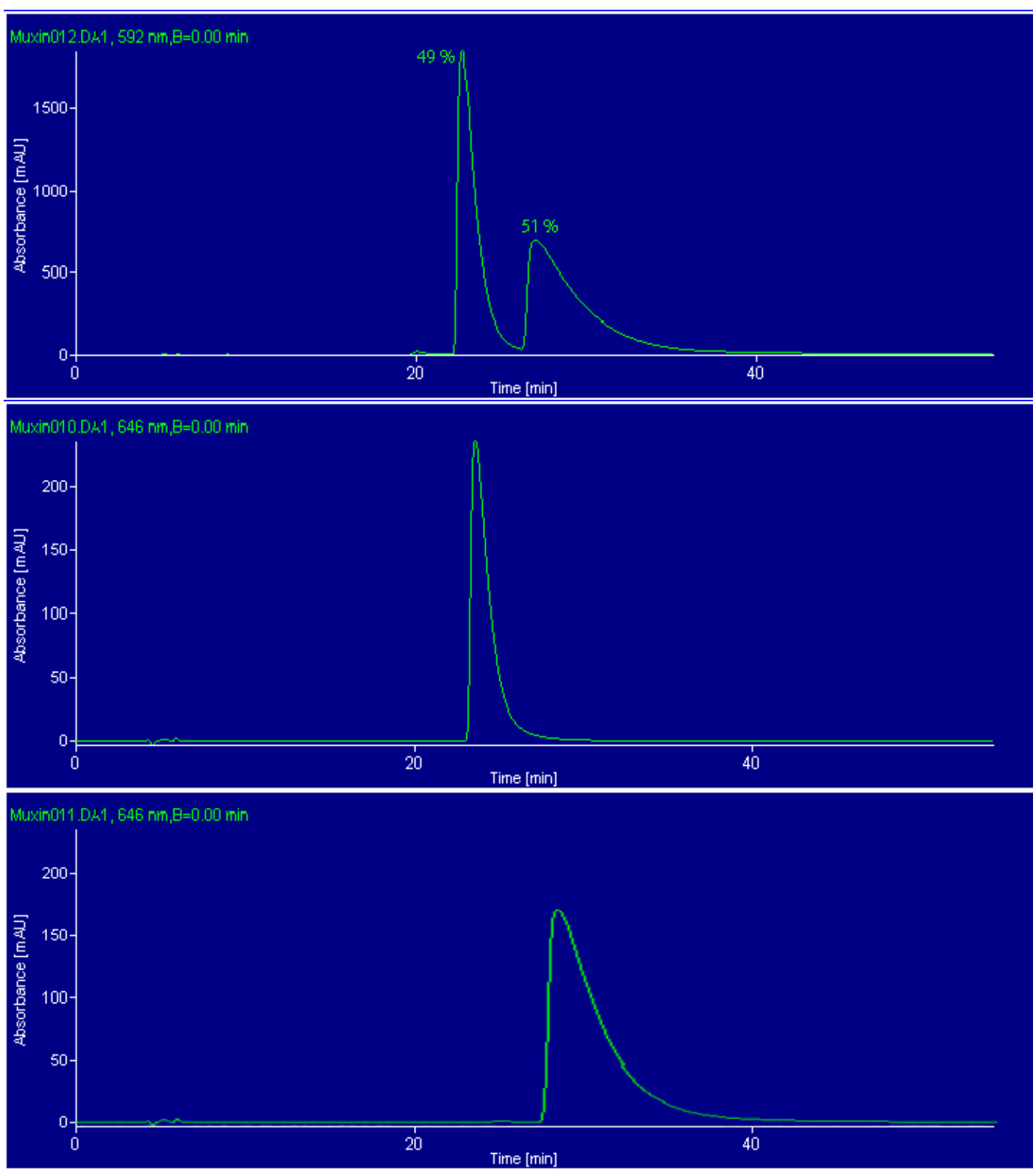

Figure 6-32 Chiral HPLC chromatograms of a) the racemic $c$ - $\mathbf{L}^{2}$ before the chiral HPLC separation, b) $(R, R)-c-\mathbf{L}^{2}$ and c) $(S, S)-c$ - $\mathbf{L}^{2}$. Chromatographic column: (Daicel IA. Mobile phase: Hexan/isopropanol $/ \mathrm{CH}_{2} \mathrm{Cl}_{2}=94: 3: 3$. Run time: $60 \mathrm{~min}$. Flow rate: $0.8 \mathrm{~mL} / \mathrm{min}$ ).

\subsubsection{Molecular modelling results}

Density functional theory (DFT) optimizations of the $\operatorname{Pd}_{3}\left(o-\mathbf{L}^{2}\right)_{6}$ geometries were performed Figure 6-9a at the dispersion-corrected $\omega B$ 97XD[47]/def2SVP[48] level using Gaussian09.[49] The calculation converged the three-ring into a structure with $C_{3}$-symmetry, in which one thiophene of each ligand points its methyl substituent towards the outer rim whereas the methyl group of the other arm points to the outside of the sidewalls. However only one set of ${ }^{1} \mathrm{H}$ NMR signals was obtained for the corresponding protons with the observation of each ligand's desymmetrization. Therefore, a fast flipping of all six ligands 
should be exist, which results a fast interconversion of the whole ring between two energetically degenerate enantiomeric forms. Semiempirical calculations (PM6 level) ${ }^{[50]}$ delivered a barrier of about $6 \mathrm{kcal} \cdot \mathrm{mol}^{-1}$ for a concerted flipping pathway via a $D_{3^{-}}$ symmetric transition state (Figure 6-35b). This estimate is in accordance with the observation of only slight line broadening effects upon measuring the ${ }^{1} \mathrm{H}$ NMR spectra at temperatures down to $238 \mathrm{~K}$ (Figure 6-33).

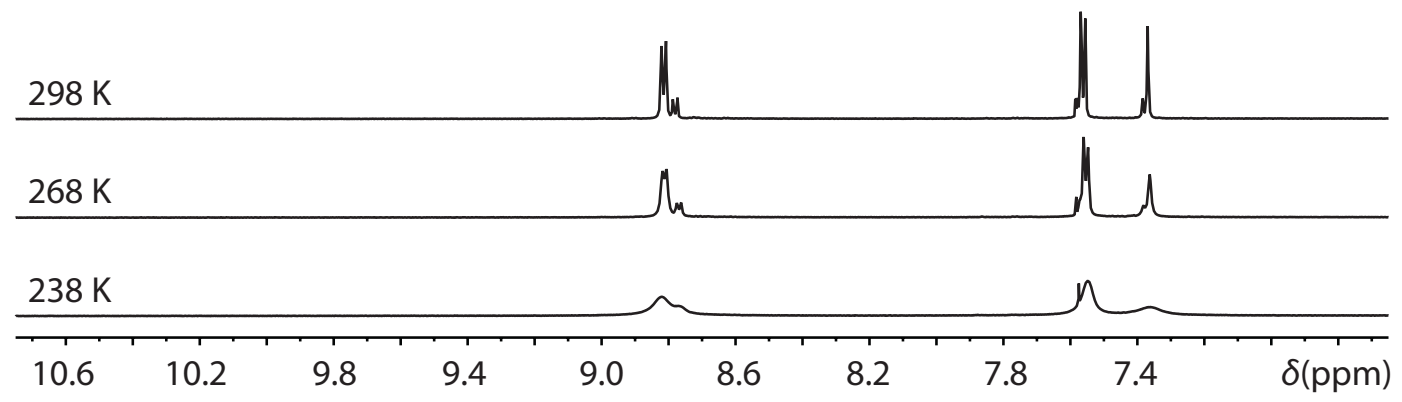

Figure 6-33 ${ }^{1} \mathrm{H}$ NMR spectra $\left(500 \mathrm{MHz}, \mathrm{CD}_{3} \mathrm{CN}\right)$ of $\mathrm{Pd}_{3}\left(o-\mathbf{L}^{2}\right)_{6}+\mathrm{Pd}_{4}\left(o-\mathbf{L}^{2}\right)_{8}$ at $298 \mathrm{~K}, 268 \mathrm{~K}$ and $238 \mathrm{~K}$.

Furthermore, the geometry optimizations and frequency calculations of $\operatorname{Pd}_{3}\left(o-\mathbf{L}^{2}\right)_{6}$ were carried out ${ }^{9}$ using the semiempirical method PM6[47] available in Gaussian09[49] in order to estimate the flipping dynamics of $\operatorname{Pd}_{3}\left(o-\mathbf{L}^{2}\right)_{6}$. In the fully optimized $C_{3}$-symmetric structure, the dihedral angles $\theta_{1}$ and $\theta_{2}$ (Figure 6-34) of both arms of each $o$ - $\mathbf{L}^{2}$ ligand in $\operatorname{Pd}_{3}\left(o-\mathbf{L}^{2}\right)_{6}$ are $139.94^{\circ}$ and $88.31^{\circ}$. Complete optimization was further verified by performing frequency calculation, which gave no imaginary frequencies.

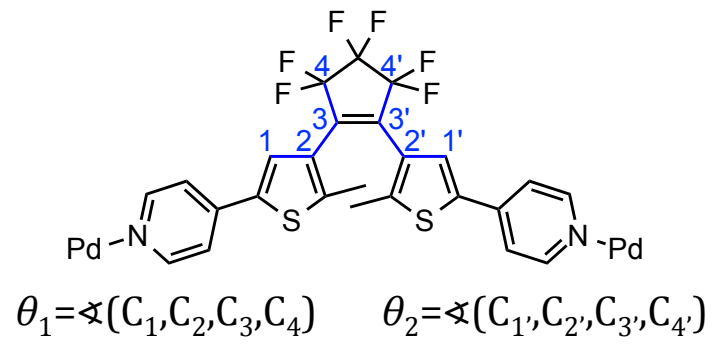

Figure 6-34 Dihedral angles $\theta_{1}$ and $\theta_{2}$ between thiophene and cyclopentene rings in $o-\mathbf{L}^{2}$.

In the proposed pathway, all six $o-\mathbf{L}^{2}$ ligands in $\operatorname{Pd}_{3}\left(o-\mathbf{L}^{2}\right)_{6}$ undergo rotation in a concerted fashion. The $o-\mathbf{L}^{2}$ ligand arms with $\theta_{1}=139.94^{\circ}$ will rotate to $88.31^{\circ}$, at the same time those

${ }^{9}$ Goemetry optimizations and frequency calculations were carried out by Anex Jose. 
arms with $\theta_{2}=88.31^{\circ}$ will rotate to $139.94^{\circ}$. Optimizations of intermediate geometries for this pathway were performed by constraining all the dihedral angles of the arms of ligand to values calculated manually (step increment of $5.17^{\circ}$, by dividing the total angle of rotation for an arm into smaller steps, Figure 6-36a). The highest energy structure in this pathway, the transition state is of $D_{3}$-symmetry and the energy barrier for this proposed pathway is $6 \mathrm{kcal} \cdot \mathrm{mol}^{-1}$ (Figure 6-36b). Both initial and final structures possess $C_{3}$ symmetry (Figure 6-35).

a)

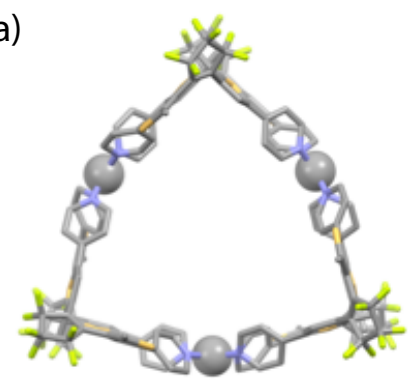

b)

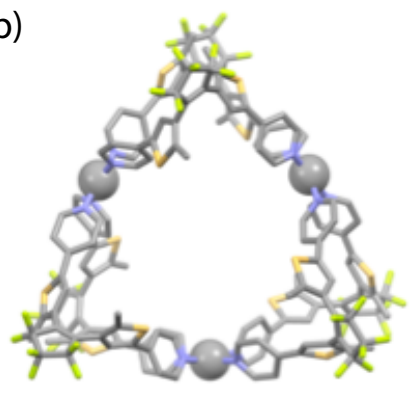

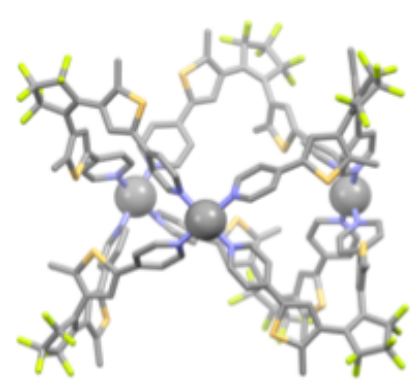
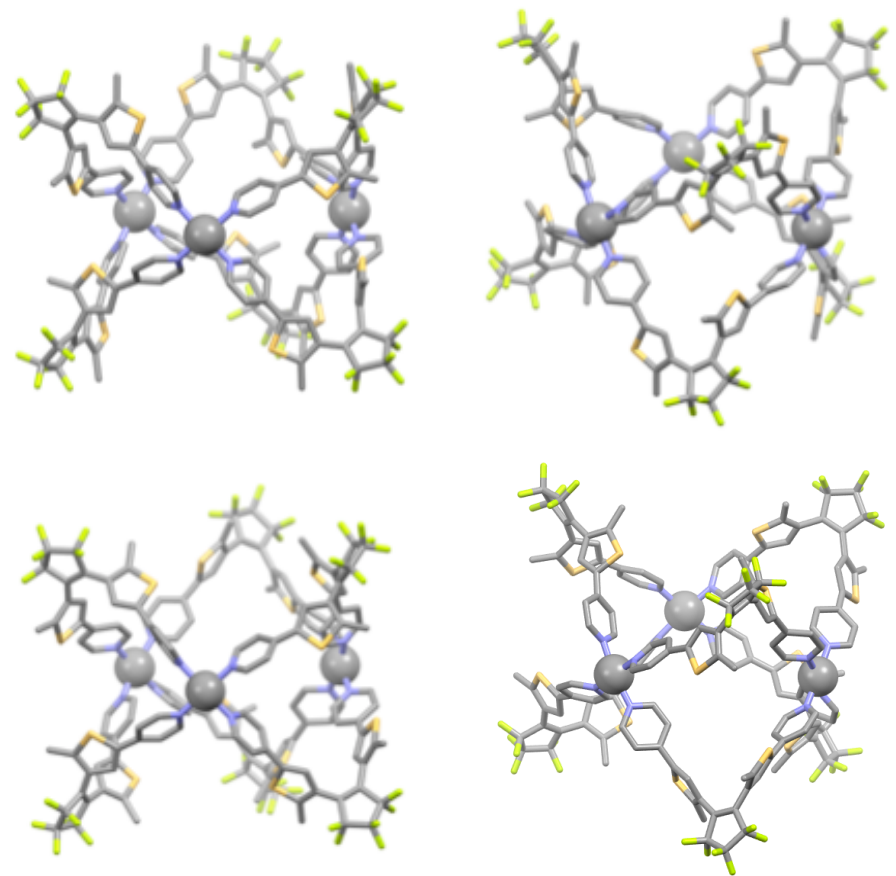

Figure 6-35 Different views of a) the initial and final PM6 structure of $\operatorname{Pd}_{3}\left(o-\mathbf{L}^{2}\right)_{6}$ and b) the transition state of $\operatorname{Pd}_{3}\left(o-\mathbf{L}^{2}\right)_{6}$.

a)

\begin{tabular}{|c|c|c|c|}
\hline Step & $\boldsymbol{\theta}_{\mathbf{1}}\left(^{\circ}\right)$ & $\boldsymbol{\theta}_{\mathbf{2}}\left({ }^{\circ}\right)$ & $\boldsymbol{\Delta E}_{\text {ZPE }}(\mathbf{k c a l} / \mathbf{m o l})$ \\
\hline 1 & 139.94 & 88.31 & 0 \\
\hline 2 & 134.77 & 93.47 & 0.44992467 \\
\hline 3 & 129.61 & 98.63 & 1.69866957 \\
\hline 4 & 124.45 & 103.80 & 3.48142548 \\
\hline 5 & 119.28 & 108.96 & 5.29430187 \\
\hline 6 & 114.12 & 114.12 & 6.01280082 \\
\hline 7 & 108.96 & 119.28 & 5.29430187 \\
\hline 8 & 103.80 & 124.45 & 3.48142548 \\
\hline 9 & 98.63 & 129.61 & 1.69804206 \\
\hline 10 & 93.47 & 134.77 & 0.44866965 \\
\hline 11 & 88.31 & 139.94 & 0 \\
\hline
\end{tabular}

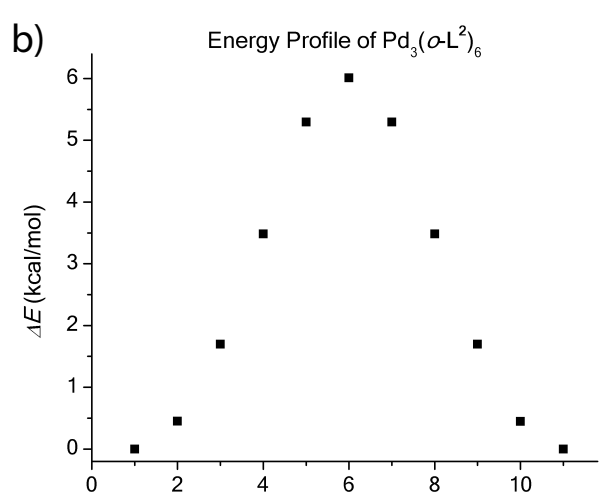

Figure 6-36 a) Summary of the steps and angles variation and the zero point energy of the ligand, b) Energy profile of $\operatorname{Pd}_{3}\left(o-\mathbf{L}^{2}\right)_{6}$ at PM6 level. 
The structure of smaller sphere $\operatorname{Pd}_{12}\left(c-\mathbf{L}^{2}\right)_{24}$ was optimized on the semiempirical PM6 level of theory. The result is shown in Figure 6-37.


Figure 6-37 Different views of the PM6 structure $\operatorname{Pd}_{12}\left(c-\mathbf{L}^{2}\right)_{24}$.

\subsection{References and Notes}

[1] A. J. McConnell, S. C. Wood, P. P. Neelakandan, J. R. Nitschke, Chem. Rev. 2015, 115, 7729.

[2] T. Kudernac, N. Ruangsupapichat, M. Parschau, B. Maciá, N. Katsonis, S. R. Harutyunyan, K.-H. Ernst, B. L. Feringa, Nature 2011, 479, 208.

[3] X. Zhang, Z. Chi, Y. Zhang, S. Liu, J. Xu, J. Mater. Chem. C 2013, 1, 3376.

[4] A. Tsuda, Y. Nagamine, R. Watanabe, Y. Nagatani, N. Ishii, T. Aida, Nature Chem. 2010, $2,977$.

[5] M. Zhang, D. Xu, X. Yan, J. Chen, S. Dong, B. Zheng, F. Huang, Angew. Chem. Int. Chem. 2012, 51, 7011.

[6] S. Bivaud, J.-Y. Balandier, M. Chas, M. Allain, S. Goeb, M. Salle, J. Am. Chem. Soc. 2012, 134, 11968.

[7] Q. Gan, T. K. Ronson, D. A. Vosburg, J. D. Thoburn, J. R. Nitschke, J. Am. Chem. Soc. 2015, 137, 1770.

[8] A. Gasnier, J.-M. Barbe, C. Bucher, F. Denat, J.-C. Moutet, E. Saint-Aman, P. Terech, G. Royal, Inorg. Chem. 2008, 47, 1862.

[9] S. Chen, L.-J. Chen, H.-B. Yang, H. Tian, W. Zhu, J. Am. Chem. Soc. 2012, 134, 13596.

[10] S.-C. Wei, M. Pan, Y.-Z. Fan, H. Liu, J. Zhang, C.-Y. Su, Chem. Eur. J. 2015, 21, 7418.

[11] T. Murase, S. Sato, M. Fujita, Angew. Chem. Int. Ed. 2007, 46, 5133.

[12] Y. Hua, A. H. Flood, J. Am. Chem. Soc. 2010, 132, 12838.

[13] J. Park, L.-B. Sun, Y.-P. Chen, Z. Perrz, H.-C. Zhou, Angew. Chem. Int. Ed. 2014, 53, 5842.

[14] T. Sakano, T. Ohashi, M. Yamanaka, M., K. Kobayashi, Org. Biomol. Chem. 2015, 13, 8359. 
[15] M. Irie, T. Fukaminato, K. Matsuda, S. Kobatake, Chem. Rev. 2014, 114, 12174.

[16] A. Fihey, A. Perrier, W. R. Browne, D. Jacquemin, Chem. Soc. Rev. 2015, 44, 3719.

[17] M. Han, R. Michel, B. He, Y.-S. Chen, D. Stalke, M. John, G. H. Clever, Angew. Chem. Int. Ed. 2013, 52, 1319.

[18] S.-S. Sun, J. A. Anspach, A. J. Lees, Inorg. Chem. 2002, 41, 1862.

[19] X. Yan, J.-F. Xu, T. R. Cook, F. Huang, Q.-Z. Yang, C.-H. Tung, P. J. Stang, Proc. Nat. Acad. Sci. 2014, 111, 8717.

[20] T. Hirose, F. Helmich, E. W. Meijer, Angew. Chem. Int. Ed. 2012, 52, 304.

[21] J. Kärnbratt, M. Hammarson, S. Li, H. L. Anderson, B. Albinsson, J. Andréasson, Angew. Chem. Int. Ed. 2010, 49, 1854.

[22] T. B. Norsten, N. R. Branda, Adv. Mater. 2001, 13, 347.

[23] U. Pischel, J. Andréasson, New J. Chem. 2010, 34, 2701.

[24] A. Presa, R. F. Brissos, A. B. Caballero, I. Borilovic, L. Korrodi-Gregório, R. PérezTomás, O. Roubeau, P. Gamez, Angew. Chem. Int. Ed. 2015, 54, 4561.

[25] J. Park, D. Feng, S. Yuan, H.-C. Zhou, Angew. Chem. Int. Ed. 2015, 54, 430.

[26] D. E. Williams, J. A. Rietman, J. M. Maier, R. Tan, A. B. Greytak, M. D. Smith, J. A. Krause, N. B. Shustova, J. Am. Chem. Soc. 2014, 136, 11886.

[27] H. Kai, S. Nara, K. Kinbara, T. Aida, J. Am. Chem. Soc. 2008, 130, 6725.

[28] Y. Hotta, S. Fukushima, J. Motoyanagi, A. Tsuda, Chem. Commun. 2015, 51, 2790.

[29] M. Han, D. M. Engelhard, G. H. Clever, Chem. Soc. Rev. 2014, 43, 1848.

[30] P. J. Stang, J. A. Whiteford, Organometallics 1994, 13, 3776.

[31] M. Fujita, J. Yazaki, K. Ogura, J. Am. Chem. Soc. 1990, 112, 5645.

[32] M. Fujita, J. O. Sasaki, T. Mitsuhashi, T. Fujita, J. Yazaki, K. Yamaguchi, K. Ogura, Chem. Commun. 1996, 1535.

[33] A. Sautter, D. G. Schmid, G. Jung, F. Wurthner, J. Am. Chem. Soc. 2001, 123, 5424.

[34] K. Suzuki, M. Kawano, M. Fujita, Angew. Chem. Int. Ed. 2007, 46, 2819.

[35] T. Weilandt, R. W. Troff, H. Saxell, K. Rissanen, C. A. Schalley, Inorg. Chem. 2008, 47, 7588.

[36] C. S. Campos-Fernández, B. L. Schottel, H. T. Chifotides, J. K. Bera, J. Bacsa, J. M. Koomen, D. H. Russell, K. R. Dunbar, J. Am. Chem. Soc. 2005, 127, 12909.

[37] K. Harris, D. Fujita, M. Fujita, Chem. Commun. 2013, 49, 6703.

[38] Q. F. Sun, J. Iwasa, D. Ogawa, Y. Ishido, S. Sato, T. Ozeki, Y. Sei, K. Yamaguchi, M. Fujita, Science 2010, 328, 1144.

[39] J. Bunzen, J. Iwasa, P. Bonakdarzadeh, E. Numata, K. Rissanen, S. Sato, M. Fujita, Angew. Chem. Int. Ed. 2012, 51, 3161.

[40] H. Yokoyama, Y. Ueda, D. Fujita, S. Sato, M. Fujita, Chem. Asian J. 2015, 10, 2292.

[41] C. Gütz, R. Hovorka, C. Klein, Q.-Q. Jiang, C. Bannwarth, M. Engeser, C. Schmuck, W. Assenmacher, W. Mader, F. Topić, K. Rissanen, S. Grimme, A. Lützen, Angew. Chem. Int. Ed. 2014, 53, 1693.

[42] B. Sampedro, P. Crespo, A. Hernando, R. Litrán, J. C. Sánchez López, C. López Cartes, A. Fernandez, J. Ramírez, J. González Calbet, M. Vallet, Phys. Rev. Lett. 2003, 91, 237203.

[43] Y. Luo, M. Esseling, M. Münzenberg, K. Samwer, New. J. Phys. 2007, 9, 329. 
[44] S. Fraysse, C. Coudret, J. P. Launay, Eur. J. Inorg. Chem. 2000, 1581.

[45] B. He, O. S. Wenger, J. Am. Chem. Soc. 2011, 133, 17027.

[46] David R. Lide, Ed., CRC Handbook of Chemistry and Physics, 89th Edition (Internet Version 2009), CRC Press/Taylor and Francis, Boca Raton, FL.

[47] J.-D. Chai, M. Head-Gordon, Phys. Chem. Chem. Phys. 2008, 10, 6615.

[48] F. Weigend, R. Ahlrichs, Phys. Chem. Chem. Phys. 2005, 7, 3297.

[49] Gaussian 09, Rev. D.; M. J. Frisch, G. W. Trucks, H. B. Schlegel, G. E. Scuseria, M. A. Robb, J. R. Cheeseman, G. Scalmani, V. Barone, B. Mennucci, G. A. Petersson, H. Nakatsuji, M. Caricato, X. Li, H. P. Hratchian, A. F. Izmaylov, J. Bloino, G. Zheng, J. L. Sonnenberg, M. Hada, M. Ehara, K. Toyota, R. Fukuda, J. Hasegawa, M. Ishida, T. Nakajima, Y. Honda, O. Kitao, H. Nakai, T. Vreven, J. A. Montgomery, Jr., J. E. Peralta, F. Ogliaro, M. Bearpark, J. J. Heyd, E. Brothers, K. N. Kudin, V. N. Staroverov, R. Kobayashi, J. Normand, K. Raghavachari, A. Rendell, J. C. Burant, S. S. Iyengar, J. Tomasi, M. Cossi, N. Rega, J. M. Millam, M. Klene, J. E. Knox, J. B. Cross, V. Bakken, C. Adamo, J. Jaramillo, R. Gomperts, R. E. Stratmann, O. Yazyev, A. J. Austin, R. Cammi, C. Pomelli, J. W. Ochterski, R. L. Martin, K. Morokuma, V. G. Zakrzewski, G. A. Voth, P. Salvador, J. J. Dannenberg, S. Dapprich, A. D. Daniels, O. Farkas, J. B. Foresman, J. V. Ortiz, J. Cioslowski, and D. J. Fox, Gaussian, Inc., Wallingford CT, 2013.

[50] J. J. P. Stewart, J. Mol. Model. 2007, 13, 1173. 


\section{SUMMARY}

An investigation into the self-assembly and the host-guest chemistry of PdII-based metalorganic architectures was presented in this dissertation.

In Chapter 2, the formation of an inclusion complex of the hexamolybdate anion $\left[\mathrm{Mo}_{6} \mathrm{O}_{19}\right]^{2-}$ inside a cationic $\mathrm{Pd}_{2} \mathbf{L}_{4}$ coordination cage in solution was described. Upon crystallization, a conversion of the host-guest complex $\left\{\left[\mathrm{Mo}_{6} \mathrm{O}_{19}\right] @ \mathrm{Pd}_{2} \mathbf{L}_{4}\right\}$ into a new supramolecular aggregate $\left\{\left[\mathrm{Mo}_{6} \mathrm{O}_{19}\right] @ \mathbf{L}_{3}+2 \mathrm{H}\right\}$ was observed. The strategies for the formation of the inclusion complex and the aggregate after structure conversion were explained and comprehensively characterized both in solution and in the solid state. The X-ray structure of $\left\{\left[\mathrm{Mo}_{6} \mathrm{O}_{19}\right] @ \mathbf{L}_{3}+2 \mathrm{H}\right\}$ reveals that one $\left[\mathrm{Mo}_{6} \mathrm{O}_{19}\right]^{2-}$ anion is wrapped in a chiral, cyclic arrangement by three ligands, in the absence of any PdII cations. Additionally, two of the six pyridines of the ligands are protonated. Surprisingly, the aggregate structure is stabilized by non-covalent interactions, including a number of $\mathrm{C}-\mathrm{H} \cdots \mathrm{O}_{\mathrm{Mo}}$ contacts and a circular arrangement of short interligand $\mathrm{CF}_{3}-$ pyridine ring $_{\text {contacts. This non-covalent }}$ approach of covering an inorganic cluster with organic compounds may lead to the generation of hybrid materials with new properties and functions.

In the following Chapter $\mathbf{3}$, a conversion of a cubic $\operatorname{Pd}_{6} \mathbf{L}_{12}{ }_{12}$ into a square-cuboid $\operatorname{Pd}_{6} \mathbf{L}^{2}{ }_{8}$ box was described. A mathematical derivation was first applied, based on the arm lengths and angles of tripodal ligands, in order to find out under which geometric condition a structure with reduced symmetry such as a square-cuboid box could be formed. After computational assisted design, a $90^{\circ}$-angled bis-pyridyl ligand $\mathbf{L}^{\mathbf{1}}$ and a tripodal tris-pyridyl ligand $\mathbf{L}^{2}$ were synthesized. The complexation reaction of the precisely designed ligands with $\mathrm{Pd}^{\mathrm{II}}$ cations, led to clean quantitatively formation of a cubic $\operatorname{Pd}_{6} \mathbf{L}_{12}$ and a square-cuboid $\operatorname{Pd}_{6} \mathbf{L}^{2}{ }_{8}$, respectively, without any unproductive ligand-site scrambling. Both of the cages were 
successfully characterized by NMR spectroscopy and ESI-MS, and the formations were supported by molecular modeling as well. Especially attractive in the structure of the square-cuboid box is, that all six faces, top and bottom two squares and four side rectangles, are constructed cleanly from simple $\mathrm{Pd}$ (pyridine) ${ }_{4}$ complexes. Neither heteronuclear nor heteroleptic approach is needed. These results are envisioned as a step forward towards the development of supramolecular structures with lower symmetry by simple ligand design.

Moreover, in Chapter 4, a new coordination $\operatorname{Pd}_{2} \mathbf{L}_{4}$ cage was prepared based on lightswitchable dithienylethene (DTE) units in which all four ligands can be cleanly switched between a conformationally flexible open-form and a rigid closed-form. As can be seen clearly in DOSY NMR, the hydrodynamic radii of both open- and closed-cages are distinguishable, which could modulate the binding affinity reversibly for the spherical $\left[\mathrm{B}_{12} \mathrm{~F}_{12}\right]^{2-}$ guest. Simple irradiation at different wavelengths, the uptake and release of guests from the cage interior could be controlled. The system has been extensively characterized by high-resolution NMR, UV-Vis spectroscopy, ESI-FTICR mass spectrometry, and by a synchrotron X-ray crystallography of the open-form cage. In addition, the thermodynamic parameters of the encapsulation of the guest in both photoisomeric cages were determined by van't Hoff analysis. The high photo responsiveness of the cage itself, and the alterable binding affinity will provide wide applications in fields such as controlled drug release and supramolecular catalysis.

Finally in Chapter 5, a similar light-switchable bidentate pyridyl ligand based on the same DTE units as described in Chapter $\mathbf{4}$ was used to trigger a clean structural interconversion between coordination self-assembled structures with tremendous change in shape, size and nuclearity. The open-formed ligand could form three- and four-membered rings with stoichiometric amount of $\mathrm{Pd}^{\mathrm{II}}$. The presence of different templating anions has an influence on the product formation, if only the three-ring was formed, or a mixture of three- and four-ring. Whereas the closed-formed rigid ligand, was observed to form large $\operatorname{Pd}_{24} \mathbf{L}_{48}$ rhombicuboctahedral spheres with a molecular weight of 31802 Da including all counter anions. Although no X-ray structures was obtained for this light-sensitive structures, the formation of the rings and spheres were confirmed by detailed NMR spectroscopic analyses, CSI-MS spectrometry, AFM, TEM and GISAXS both in solution and on surfaces. The totally different photoswitching rates of the rings and spheres are remarkably dissimilar. By altering the irradiation wavelength, the transformation of small rings into the large sphere was found to be extremely slow, in contrast the large sphere could be reconverted back into small rings rapidly upon irradiation, by a sequence of fast photo-induced ring-opening reactions to give a tentative open-form sphere and 
disintegrates under thermal rearrangement to the entropically favored small rings. These results provide a platform to investigate the stimuli-responsive structural reorganization processes, to design artificial multicomponent complexes closer to the massive scale of biological self-assemblies, to understand the interactions between macromolecules, such as protein-protein interactions.

Overall, special emphasis has been paid in this work on the construction of lower symmetric self-assemblies by rational ligand design, the light-responsive structure conversion process, and the host-guest chemistry as well as the synthesis and characterization of new self-assemblies. The results described in this work showed that the future of self-assembled systems is still attractive, although in each case one sort of ligands are coordinated with one kind of metal ion. The complexity of these systems does not approach that of many complicated processes in nature. However, these simpler systems have played very important roles in developing and expanding the supramolecular chemistry field. By starting from such simple systems step by step, where the introduction of different functionalities could be more selectively, the interactions between molecules can be studied more precisely and individually, useful applications on the basis of more complex structures will be widely exploited. 
8 APPENDIX 


\section{APPENDIX 1 LIST OF ABBREVIATIONS}

2D

3D

$\mathrm{NBu}_{4}$

$\mathrm{CDCl}_{3}$

$\mathrm{CD}_{3} \mathrm{CN}$

CSI-MS

COSY

DCM

DFT

$\mathrm{D}_{2} \mathrm{O}$

DTE

DOSY

$e e$

eq

equiv

ESI-MS

HSQC

HMBC

HRMS

$\min$

$\mathrm{mM}$

MOF

$m / z$

NMR

NOESY

ppm

THF two-dimensional

three-dimensional

tetrabutylammonium

deuterated chloroform

deuterated acetonitrile

cryospray ionization mass spectroscopy

correlated spectroscopy

dichloromethane

density functional theory

deuterated water

dithienylethene

diffusion-ordered Spectroscopy

enantiomeric excess

equation

equivalent

electrospray ionisation mass spectrometry

heteronuclear single quantum correlation

heteronuclear multiple bond correlation

high resolution mass spectrometry

minute

millimolar

metal-organic framework

mass-to-charge ratio

nuclear magnetic resonance

nuclear overhauser effect spectroscopy

parts per million

tetrahydrofuran 


\section{APPENDIX 2 GENERAL AND INSTRUMENTATION}

\section{General experimental procedures}

All air and/or water sensitive reactions were performed under a nitrogen atmosphere using standard Schlenk line techniques, and monitored by TLC, or ${ }^{1} \mathrm{H}$ NMR spectroscopy. Tetrahydrofuran was dried over sodium, and distilled prior to use. Otherwise, commercially available solvents in p.a. or HPLC grade were used for the synthesis. All reagents were purchased from ABCR, Sigma Aldrich, TCI and Acros and used without further purification. Thin-layer-chromatography was performed on Merck aluminiumbased plates with silica gel and fluorescent indicator $254 \mathrm{~nm}$.

\section{Nuclear magnetic resonance spectroscopy}

NMR spectra were recored using Bruker Avance 300, Bruker Avance III 300, 400, Bruker Avance III HD 400, 500, or Bruker Avance I $900 \mathrm{MHz}$ spectrometers. ${ }^{13} \mathrm{C}$ and ${ }^{19} \mathrm{~F}$ spectra were measured in a proton-decoupled mode. The chemical shifts $\delta$ are given as parts per million $(\mathrm{ppm})$ referenced to the signal of the deuterated solvent $\left(\mathrm{CDCl} 3: \delta_{\mathrm{H}}=7.26 \mathrm{ppm}, \delta_{\mathrm{C}}\right.$ $=77.16 \mathrm{ppm} ; \mathrm{CD}_{3} \mathrm{CN}: \delta_{\mathrm{H}}=1.94 \mathrm{ppm}, \delta_{\mathrm{C}}=1.32,118.26 \mathrm{ppm}$ ). For the assignment of the multiplicities the following abbreviations were used: s (singlet), sbr (broad singlet), $d$ (doublet), dd (doublet of doublet), $\mathrm{t}$ (triplet), quart (quartet), m (multiplet). The coupling constants $J$ are given in $\mathrm{Hz}$.

\section{Mass spectrometry}

Mass spectrometry was performed on Bruker HCT Ultra (ESI), Bruker FTICR-MS APEX IV (HR-ESI), Bruker micrOTOF (HR-ESI), Bruker maXis (HR-ESI), Bruker MicrOTOF-QII (CSIMS). Isotopic distributions and exact masses were calculated with CambridgeSoft ChemBioDraw 13 and Bruker Compass DataAnalysis version 4.0.SP3 (Build 275).

\section{UV-Vis spectroscopy}

UV-Vis spectra were acquired using an Agilent 8453 UV-Visible Spectrophotometer with online measurement software UV-Visible ChemStation B.05.01 (9). 